\title{
Packet Scheduling in Wireless Systems using MIMO Arrays and VBLAST Architecture
}

\author{
Constantine I. Floros
}

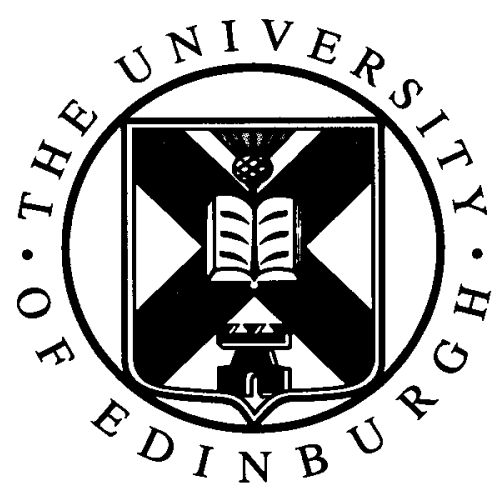

A thesis submitted for the degree of Doctor of Philosophy.

The University of Edinburgh.

April 2006 


\section{Abstract}

The third generation (3G) and fourth generation (4G) of mobile communications systems aim to provide enhanced voice, text and data services to the user. Two of the key requirements in these systems are higher spectral efficiency and QoS (Quality of Service). This thesis aims to examine ways of increasing the spectral efficiency of a communication system while meeting the QoS needs of the users.

One of the most prominent antenna techniques that has received a lot of research recently is multiple input multiple output (MIMO) antenna array systems, where both transmitter and receiver are equipped with multiple antenna elements exploiting space (antenna) diversity. Another interesting technology for realizing very high data rates over the rich scattering wireless channel is the VBLAST (Vertical Bell Labs Space Time) architecture. VBLAST detection is aiming to achieve the enormous information-theoretic capacities arising in a MIMO wireless fading channel. These techniques are thoroughly described, and their BER (Bit Error Rate) versus SNR (Signal-to-Noise Ratio) performance is evaluated by means of computer simulations in various channel environments and using different numbers of transmit antenna elements in the base station. These results of the techniques, along with other characteristics, are compared to examine their performance in various channel environments and investigate which technique is most suitable for each channel environment.

Next, we concentrate on round robin packet scheduling. In multiuser environments, independence of fading for different users, called multiuser diversity, can be exploited by a proper packet scheduling policy. A Packet Scheduler (PS) is aiming to effectively allocate radio resources to users in different channel conditions. The objectives are to maximize spectral efficiency and to provide fairness among users. We investigate the performance of various schemes based on the round robin scheme (RRS) and we propose some new algorithms. Computer simulation are conducted to compare the different scheduling schemes in terms of cell throughputs and outage capacities. The degree of fairness (in terms of time delay and data rates) among the users for these schemes is also investigated.

Finally, we focus on Proportional Fair (PF) scheduling. New algorithms using MIMO antennas and VBLAST architecture are proposed to improve the performance of the PF scheme. The system efficiency (in terms of cell throughput) and the QoS (in terms of time delay and data rates) achieved by these schemes are investigated through computer simulations.

The main objective of this work is to investigate the interplay of spatial multiplexing techniques (MIMO arrays and VBLAST architecture) with packet scheduling schemes, as well as the tradeoffs between the QoS provided to the end user and the achievable network throughput. 


\section{Declaration of originality}

I hereby declare that the research recorded in this thesis and the thesis itself was composed and originated entirely by myself in the School of Engineering and Electronics at the University of Edinburgh. 
to my parents and my brother. 


\section{Acknowledgements}

I would like to show my sincerest gratitude to my co-supervisors, Professor Steve McLaughlin and Dr. John.Thompson for giving me copious amounts of insightful guidance, constant encouragement, constructive criticism, and expertise on every subject that arose throughout all these years. Their enthusiasm and dedication to their students are truly inspiring. It is my very privilege to have been one of them. I would also like to thank Dr. Dave Laurenson for his help and advice.

I thank my good friend Antonios Koutalos for his valuable help when I applied to Edinburgh University for admission as a postgraduate student.

Many thanks to my friends and colleagues from the Institute for Digital Communications (former Signals and Systems Group) for countless discussions and for a pleasant working atmosphere.

I thank the School of Engineering and Electronics (formerly known as Department of Electronics and Electrical Engineering) of the University of Edinburgh for providing the financial support for this work.

I thank the IT support team of the School for their help with computer-related problems and for responding to our requests for new software and utilities that make our work easier.

Last but not least, I wish to thank my parents Ioannis-Vayios and Vasiliki and my brother Anastasios for their support and love. This work is dedicated to them. Although my father had to leave this world so suddenly and so early, I can still feel his presence. 


\section{Contents}

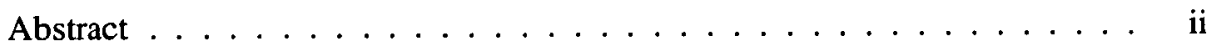

Declaration of originality ........................ iii

Acknowledgements ...................... . v

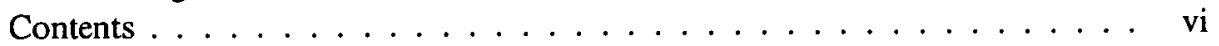

List of figures ....................... . ix

List of tables ....................... . . . . . . . . . . . . . . . . . . . .

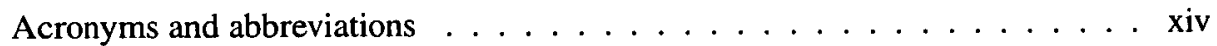

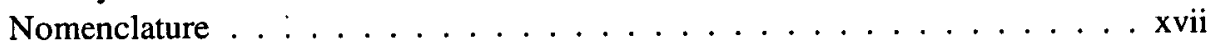

1 Introduction $\quad 1$

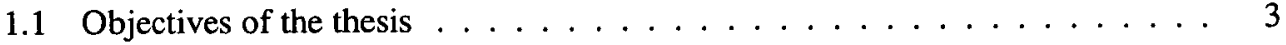

1.2 Outline of the Dissertation ................... 4

2 System model - Related Work $\quad 6$

2.1 Introduction . . . . . . . . . . . . . . . . . . 6

2.2 Wireless Communication System . . . . . . . . . . . . . . 6

2.2.1 Cellular Radio System . . . . . . . . . . . . . . . . . . . . 9

2.2.2 Transmission and multiplexing . . . . . . . . . . . . . . . . 9

2.3 High Data Rate wireless networks (HDR) . . . . . . . . . . . . . . . 13

2.3.1 Multiple-Input Multiple-Output (MIMO) system . . . . . . . . . 14

2.3.2 Information-Theoretic Results for Multiple-Antenna Channels . . . . . 16

2.3.3 Multi-User Channels ... . . . . . . . . . . . . . . . 19

2.4 Packet Scheduling Principles and Strategies . . . . . . . . . . . . . . . 21

2.4.1 Packet Scheduling Policies . . . . . . . . . . . . . . . 23

2.4.2 Packet Scheduling Algorithms ................ 24

2.5 Conclusions . . . . . . . . . . . . . . . . . . . 27

3 Physical Channel Performance $\quad 29$

3.1 Introduction . . . . . . . . . . . . . . . . . . . 29

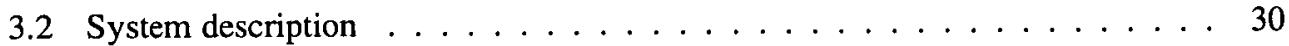

3.2 .1 Transmitter Structure . . . . . . . . . . . . . . . . . . . . 31

3.3 MIMO Channel Models . . . . . . . . . . . . . . . . . . 32

3.3.1 Non-physical MIMO Channel Models . . . . . . . . . . . . . 33

3.3.2 Physical MIMO Channel Models . . . . . . . . . . . . . 36

3.3.3 Remarks on MIMO Channel Models . . . . . . . . . . . . . . . . 38

3.4 Stochastic MIMO Channel Model . . . . . . . . . . . . . . . . . . . . . 39

3.5 Generation of Simulated Correlated Channel Coefficients . . . . . . . . . . . 41

3.6 Detection Algorithms . . . . . . . . . . . . . . . . . . . 42

3.6.1 Zero-Forcing Equalizer $(\mathrm{ZF}) \ldots \ldots \ldots$. . . . . . . . . . . . . . . . . . . . 42

3.6.2 Minimum Mean Squared Error Detector (MMSE) . . . . . . . . . . . . 43

3.6.3 VBLAST Detection Algorithm ................ 43

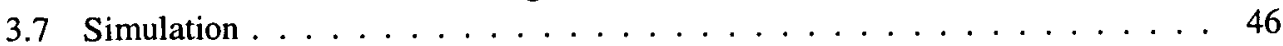


3.7.1 Statistical Confidence . . . . . . . . . . . . . . 47

3.7 .2 Simulation Setup . . . . . . . . . . . . . . . . . . . . 49

3.7 .3 Results . . . . . . . . . . . . . . . . . . . 50

3.8 Conclusions $\ldots \ldots \ldots \ldots \ldots \ldots \ldots \ldots$

4 Exploiting Multiuser Diversity for MIMO Cellular Systems using RRS Packet Scheduling and the VBLAST Receiver $\quad 61$

4.1 Introduction to Scheduling $\ldots \ldots \ldots \ldots \ldots$

4.2 System Model . . . . . . . . . . . . . . . . . . . . . . . 62

4.2.1 Physical Channel Characteristics . . . . . . . . . . . . . . . 64

4.3 Packet Scheduling and System Capacity . . . . . . . . . . . . . . . 65

4.3.1 Round Robin Scheduling (RRS) . . . . . . . . . . . . . . . . 65

4.3.2 Antenna-Assisted Round Robin Scheduling (AA-RRS) . . . . . . . . 66

4.4 Proposed Scheduling Schemes . . . . . . . . . . . . . . 67

4.4.1 VBLAST AA-RRS Feasible Data Rates Algorithm . . . . . . . . . 67

4.4 .2 Feasible Data Rates . . . . . . . . . . . . . . . . . 71

4.4 .3 Best user schemes . . . . . . . . . . . . . . . 75

4.4.4 Antenna-Assisted (AA) MMSE fair Scheme . . . . . . . . . . . 77

4.4 .5 AA VBLAST fair Scheme . . . . . . . . . . . . . 83

4.5 Simulation Model . . . . . . . . . . . . . . . . 83

4.5 .1 Performance metrics . . . . . . . . . . . . . . 85

4.5 .2 Results . . . . . . . . . . . . . . . . 87

4.6 Conclusions . . . . . . . . . . . . . . . . 106

5 VBLAST Proportional Fair Scheme 108

5.1 Introduction . . . . . . . . . . . . . . . . . . 108

5.2 System Model and Capacity . . . . . . . . . . . . . . . . . . . 109

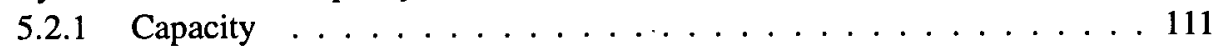

5.3 Proportional Fair Algorithm $\ldots \ldots \ldots \ldots \ldots 11$

5.3 .1 Multiuser Diversity . . . . . . . . . . . . . . . 113

5.4 Proportional Faimess Scheduling Schemes Variants . . . . . . . . . . . 113

5.4.1 Antenna Assisted Proportional Fairness MMSE single (only one user each time slot) . . . . . . . . . . . . . . . 113

5.4.2 Antenna Assisted Proportional Faimess VBLAST single (only one user each time slot) . . . . . . . . . . . . . 115

5.4.3 Antenna Assisted Proportional Faimess multi (more than one user each time slot) . . . . . . . . . . . . . . . . . 117

5.4.4 Antenna Assisted Proportional Faimess VBLAST multi (more than one user each time slot) . . . . . . . . . . . . . . . . . . 119

5.4.5 Antenna Assisted Proportional Fairness VBLAST multi fair (more than one users each time slot - but users not allowed to be assigned to multiple antennas) $\ldots \ldots \ldots \ldots \ldots \ldots . \ldots \ldots \ldots$

5.5 Simulation . . . . . . . . . . . . . . . . . . 125

5.5 .1 Results . . . . . . . . . . . . . . . . 125

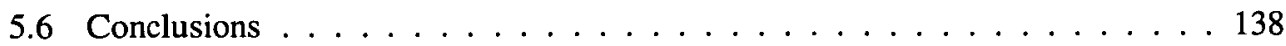

5.6 .1 Future Work . . . . . . . . . . . . . . . . . . . 140 
6 Summary - Conclusions 141

6.1 Summary and thesis contributions . . . . . . . . . . . . . 141

6.2 Limitations of the work and scope for further research . . . . . . . . . . . 144

$\begin{array}{ll}\text { References } & 145\end{array}$

$\begin{array}{ll}\text { A Pseudocodes for Chapter 4 } & 156\end{array}$

B Pseudocodes for Chapter $5 \quad 162$

$\begin{array}{ll}\text { C Publications } & 166\end{array}$ 


\section{List of figures}

2.1 Radio Propagation Scenario $\ldots \ldots \ldots \ldots$

2.2 Cellular radio system . . . . . . . . . . . . . . . . . 9

2.3 FDMA where different channels are assigned different frequency bands. . . . . 10

2.4 Multiple access schemes: (a)TDMA scheme where each channel occupies a cyclically repeating time slot, (b) Spread spectrum multiple access in which each channel is assigned a unique PN code which is orthogonal or approximately orthogonal to PN codes used by other users. . . . . . . . . . . . . . . . . . . . 11

2.5 MIMO wireless transmission system . . . . . . . . . . . . . . . 14

2.6 MIMO channel communication links . . . . . . . . . . . . . . . . . 19

3.1 High-level view of a MIMO wireless communication system. . . . . . . . . . 30

3.2 Diagram of MIMO wireless transmitter. The transmitter is equipped with $M$ antenna elements. Coding, modulation, and mapping of the signals onto the the antennas is realized separately. . . . . . . . . . . . . . . . 32

3.3 Sketch of a scenario where all scatterers are located near the MS so the impinging field at the BS is confined to a narrow azimuth region with a well defined mean directionof-arrival. . . . . . . . . . . . . . . . . . 36

3.4 Sketch of a two ring scenario where both the BS and MS are surrounded by scatterers. 37

3.5 Illustration of the distributed scattering model. . . . . . . . . . . . . . . . 39

3.6 Flow chart illustrating the practical procedure to obtain correlated channel coefficient. . 41

3.7 Block diagram of channel with zero-forcing equalizer. . . . . . . . . . . 42

3.8 Numbers in blocks represent the layer that can transmit its symbols at that antenna and symbol period. Filled blocks represent space time wastage: (a) D-BLAST: diagonal layering, (b) VBLAST: horizontal layering . . . . . . . . . . . . . . . 44

3.9 Constellation diagram for: (a) Gray-coded 16-QAM, (b) Gray-coded BPSK . . . . . . 51

3.10 Comparison of MIMO systems for different modulation schemes (BPSK vs. 16-QAM):

(a) MMSE detector, (b) VBLAST MMSE detector . . . . . . . . . . . . . . 52

3.11 Detection techniques comparison on MIMO $(4,4)$ system: (a) BPSK modulation MMSE/VBLAST MMSE detector, (b) 16-QAM modulation - MMSE/VBLAST MMSE detector . . . . . . . . . . . . . . . . . 52

3.12 Comparison of MIMO systems with different number of antenna arrays at transmitter and receiver, BPSK modulation, MMSE detector . . . . . . . . . . 53

3.13 Effect of Error Propagation on MIMO (4,4): (a) BPSK modulation - MMSE/VBLAST MMSE detector, (b) 16-QAM modulation - MMSE/VBLAST MMSE detector . . . . 55

3.14 Effect of Error Propagation on a $(4,4)$ MIMO 16-QAM under two different detection techniques: MMSE/VBLAST MMSE . . . . . . . . . . . . . . .

3.15 Effect of spatial fading complex correlation on a $(4,4)$ MIMO system: (a) 16-QAM modulation - VBLAST MMSE detector, (b) BPSK modulation - MMSE/VBLAST MMSE detector . . . . . . . . . . . . . . . .

3.16 Comparison of the results of the measured correlation matrices with the results of the simulated correlation matrices . . . . . . . . . . . . . . . 57 
3.17 Effect of spatial fading complex correlation on BPSK MIMO systems with different sizes of antenna arrays at transmitter and receiver: (a) MMSE detector, (a) VBLAST MMSE detector . . . . . . . . . . . . . . . 58

4.1 Aerial view of the cell. . . . . . . . . . . . . . . . 63

4.2 Downlink MIMO cellular system. . . . . . . . . . . . . . 63

4.3 AA-RRS-VBLAST algorithm . . . . . . . . . . . . . . . 69

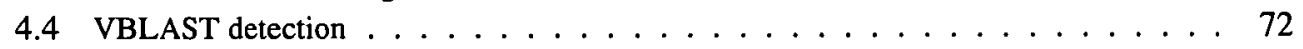

4.5 Allocation scenario for a $(2,2)$ MIMO system. . . . . . . . . . . . 73

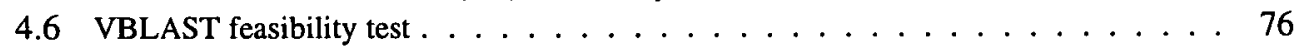

4.7 AA-RRS best user algorithm $\ldots \ldots \ldots \ldots \ldots \ldots \ldots \ldots$

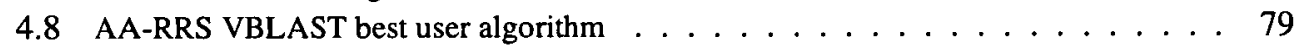

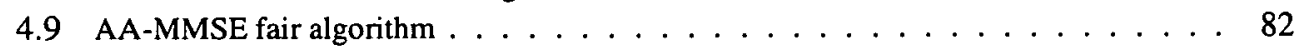

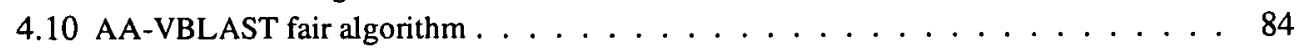

4.11 delay scenario for 3 users . . . . . . . . . . . . . . . . . 86

4.12 System capacities on a (4,4) MIMO system: (a) AA-RRS vs AA-RRS best user, (b) AA-RRS best user vs VBLAST AA-RRS best user . . . . . . . . . . 88

4.13 Average data rates per scheduled user every time slot for a $(4,4)$ MIMO system, SNR at the boundary equals $10 \mathrm{~dB} \ldots \ldots \ldots$. . . . . . . . . . 89

4.14 Average data rates per scheduled user every four time slots for a $(4,4)$ MIMO system that serves 16 simultaneous users, SNR at the boundary equals $0 \mathrm{~dB} \quad \ldots 90$

4.15 System capacities for AA-RRS MMSE and AA-RRS VBLAST scheduling schemes on a $(4,4)$ MIMO system. . . . . . . . . . . . . . . . . . 91

4.16 System capacities on a $(4,4)$ MIMO system: (a) AA-RRS vs AA MMSE fair, (b) VBLAST AA-RRS vs AA VBLAST fair . . . . . . . . . . . . 93

4.17 System capacities for AA MMSE fair and AA MMSE fair MDS (max-delete search) scheduling schemes on a $(4,4)$ MIMO system. . . . . . . . . . . . . 94

4.18 System capacities on a MIMO system: (a) symmetrical MIMO arrays, (b) asymmetrical MIMO arrays . . . . . . . . . . . . . . . . . 95

4.19 System capacities on a MIMO system: (a) symmetrical MIMO arrays, (b) asymmetrical MIMO arrays . . . . . . . . . . . . . . . . . . . . 97

4.20 System capacities on a $(4,4)$ MIMO system for various values of SNR at the boundary: (a) AA-RRS vs AA-RRS best user, (b) AA-RRS VBLAST vs AA-RRS VBLAST best user $\ldots \ldots \ldots \ldots \ldots \ldots \ldots \ldots \ldots$

4.21 System capacities on a $(4,4)$ MIMO system for various values of SNR at the boundary: (a) AA-RRS vs VBLAST AA-RRS, (b) AA MMSE fair vs AA VBLAST fair . . . . . 99

4.22 Effect of spatial correlation on a $(4,4)$ MIMO system, the correlation (at both the BS and MS) takes values from 0.1 to $0.9 . \ldots \ldots \ldots$

4.23 Effect of spatial correlation on a $(4,4)$ MIMO system: (a) the absolute value of the correlation coefficients at the BS takes values from 0.1 to 0.9 , and remains stable at MS (0.1), (b) the absolute value of the correlation coefficients at the MS takes values from 0.1 to 0.9 , and remains stable at BS $(0.1) \ldots \ldots \ldots 1$

4.24 Effect of spatial correlation on a $(4,4)$ MIMO system, the correlation (at both the BS and MS) takes values from 0.1 to $0.9 \ldots \ldots \ldots 2 \ldots \ldots \ldots$ 
4.25 Effect of spatial correlation on a (4,4) MIMO system: (a) the absolute value of the correlation coefficients at the BS takes values from 0.1 to 0.9 , and remains stable at MS (0.1), (b) the absolute value of the correlation coefficients at the MS takes values from 0.1 to 0.9 , and remains stable at BS $(0.1) \ldots \ldots \ldots 103$

4.26 Effect of spatial correlation on a (4,4) MIMO system - 16 users, the correlation (at both the $\mathrm{BS}$ and MS) takes values from 0.1 to $0.9 \ldots \ldots \ldots$. . . . . . . . . . 104

4.27 Effect of spatial correlation on a (4,4) MIMO system - 16 users: (a) the absolute value of the correlation coefficients at the BS takes values from 0.1 to 0.9 , and remains stable at MS (0.1), (b) the absolute value of the correlation coefficients at the MS takes values from 0.1 to 0.9 , and remains stable at BS $(0.1) \ldots \ldots \ldots 105$

4.28 Effect of spatial correlation on MIMO systems of different number of transmit and receive antennas: (a) System capacities for a (2,2) MIMO system, (b) System capacities for a $(6,6)$ MIMO system. For both cases, the correlation (at both the BS and MS) takes values from 0.1 to $0.9 . \ldots \ldots \ldots \ldots$. . . . . . . . . . . . . . . . . .

4.29 Effect of spatial correlation on MIMO systems of different number of transmit and receive antennas: (a) System capacities for a (2,4) MIMO system, (b) System capacities for a $(4,6)$ MIMO system. For both cases, the correlation (at both the BS and MS) takes values from 0.1 to $0.9 . \ldots \ldots \ldots \ldots \ldots$

5.1 Downlink MIMO cellular system. . . . . . . . . . . . . . . . . 110

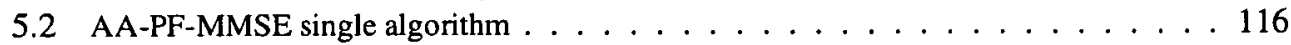

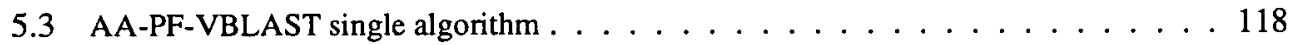

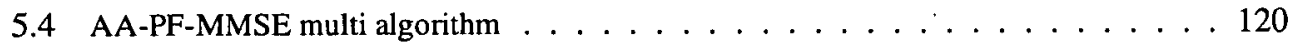

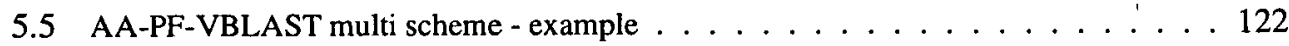

5.6 AA-PF-VBLAST multi algorithm . . . . . . . . . . . . . . . . . . 124

5.7 System capacities on a $(4,4)$ MIMO system (SNR at boundary $=0 \mathrm{~dB}$ ): (a) AA-PFMMSE single vs AA-PF-VBLAST single, (b) AA-PF-MMSE multi vs AA-PF-VBLAST

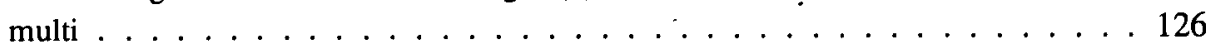

5.8 System capacities on a $(4,4)$ MIMO system (SNR at boundary = OdB): (a) AA-PFMMSE single vs AA-PF-MMSE multi, (b) AA-PF-VBLAST single vs AA-PF-VBLAST multi . . . . . . . . . . . . . . . . . . . . . . . 128

5.9 System capacities on a $(4,4)$ MIMO system $($ SNR at boundary $=0 \mathrm{~dB}) \quad \ldots \ldots \ldots$

5.10 Data Rate per user for a $(4,4)$ MIMO system (SNR at boundary $=0 \mathrm{~dB}$ ), with 20 active users: (a) AA-PF-VBLAST single vs AA-PF-VBLAST multi, (b) AA-PF-VBLAST multi vs AA-PF-VBLAST multi fair . . . . . . . . . . . . . . . . . 130

5.11 Average data rates per scheduled user every time slot for a $(4,4)$ MIMO system, SNR at the boundary equals $0 \mathrm{~dB}, 20$ active users. . . . . . . . . . 131

5.12 System capacities on a $(4,4)$ MIMO system (SNR at boundary $=0 \mathrm{~dB}) \quad \ldots \ldots 132$

5.13 System capacities on a $(4,4)$ MIMO system (SNR at boundary = OdB) $\ldots \ldots \ldots$

5.14 System capacities on a $(4,4)$ MIMO system (SNR at boundary $=0 \mathrm{~dB}$ ) - AA-PFVBLAST single scheme: (a) symmetrical MIMO arrays (b) asymmetrical MIMO arrays 134

5.15 System capacities on a $(4,4)$ MIMO system $($ SNR at boundary $=0 \mathrm{~dB})$ - AA-PFVBLAST multi scheme: (a) symmetrical MIMO arrays, (b) asymmetrical MIMO arrays 135

5.16 Time delay per scheduled user for a $(4,4)$ MIMO system (SNR at boundary $=0 \mathrm{~dB}$ ): (a) AA-PF-VBLAST single vs AA-PF-VBLAST multi, (b) AA-PF-VBLAST multi vs AA-PF-VBLAST multi fair $\ldots \ldots \ldots \ldots \ldots \ldots$ 
5.17 Effect of spatial correlation on a $(4,4)$ MIMO system with 20 active users (SNR at boundary $=0 \mathrm{~dB}$ ), the correlation (at both the BS and MS) takes values from 0.1 to 0.9: (a) AA-PF-MMSE multi vs AA-PF-VBLAST multi, (b) AA-PF-MMSE multi vs AA-PF-MMSE single . . . . . . . . . . . . . . . . . 137

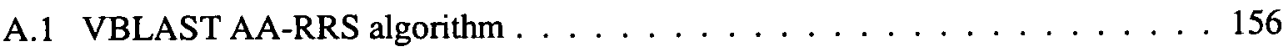

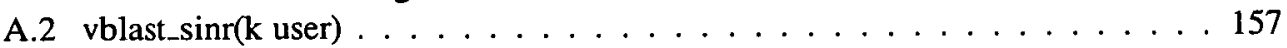

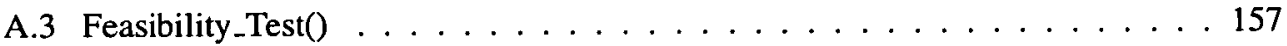

A.4 AA-RRS best user Algorithm . . . . . . . . . . . . . . 158

A.5 VBLAST AA-RRS best user Algorithm . . . . . . . . . . . . . . 159

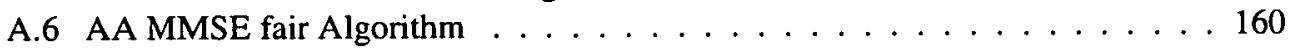

A.7 AA VBLAST fair Algorithm . . . . . . . . . . . . 161

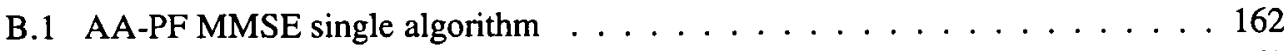

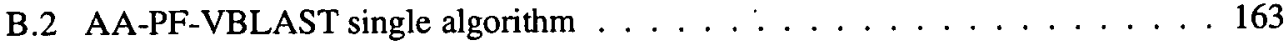

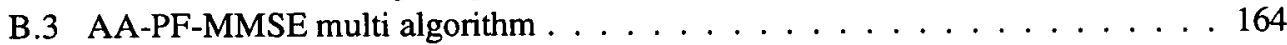

B.4 AA-PF-VBLAST multi algorithm $\ldots \ldots \ldots \ldots \ldots \ldots \ldots \ldots \ldots \ldots$ 


\section{List of tables}

4.1 Time delay per scheduled user for a $(4,4)$ MIMO systems and for different scheduling schemes in a static environment. 


\section{Acronyms and abbreviations}

$3 G$

$4 \mathrm{G}$

AWGN

AA-PF-MMSE

AA-PF-VBLAST

AA-RRS

AMC

AMPS

AOA

AOD

AP

AS

BER

BPR

BPSK

BS

BTS

$\mathrm{C} / \mathrm{I}$

$\mathrm{CDF}$

CDMA

CSI

DOA

DRC

DS

DS-SS

EP

EXS

FDD

FDMA
Third Generaration

Fourth Generaration

Additive White Gaussian Noise

Antenna Assisted Proportional Fairness MMSE

Antenna Assisted Proportional Fairness VBLAST

Antenna-Assisted Round Robin Scheduling

Adaptive Modulation and Coding

Advanced Mobile Phone Services

Angle of arrival

Angle of departure

Access Point

Angular spread

Bit Error Ratio

Branch Power Ratio

Binary Phase Shift Key

Base Station

Base Transceiver Station

Carrier-to-Interference

Cumulative Distribution Function

Code Division Multiple Access

Channel State Information

Direction of Arrival

Data Rate Control

Direct Sequence

Direct Sequence Spread Spectrum

Error Propagation

EXhaustive Search

Frequency Division Duplex

Frequency Division Multiple Access 


\begin{tabular}{|c|c|}
\hline FER & Frame Error Rate \\
\hline FTP & File Transfer Protocol \\
\hline GBSM & Geometrically-Based Stochastic Models \\
\hline GSM & Global System for Mobile communications \\
\hline HDR & High Data Rate \\
\hline HSDPA & High Speed Downlink Packet Access \\
\hline HTTP & HyperText Transfer Protocol \\
\hline IID or i.i.d. & Independent Identically-Distributed \\
\hline IP & Internet Protocol \\
\hline IS & Interim Standard \\
\hline ISI & Intersymbol Interference \\
\hline IST & Information Society Technology \\
\hline LAN & Local Area Network \\
\hline LBS & Location Based Services \\
\hline MDS & Max-Delete Search \\
\hline METRA & Multi Element Transmit Antennas \\
\hline MIMO & Multiple Input Multiple Output \\
\hline MISO & Multiple Input Single Output \\
\hline MMSE & Minimum Mean Squared Error \\
\hline MS & Mobile Station \\
\hline MUD & Multiuser Detection \\
\hline NEP & Non Error Propagation \\
\hline NLOS & Non Line-Of-Sight \\
\hline OFDM & Orthogonal Frequency-Division Multiplexing \\
\hline PAS & Power Azimuth Spectrum \\
\hline PDF & Probability Density Function \\
\hline PCS & Personal Communications Services \\
\hline PF & Proportional Faimess \\
\hline PIC & Parallel Interference Cancellation \\
\hline PRN & Pseudo Random Noise \\
\hline PS & Packet Scheduler \\
\hline RRS & Round Robin Scheme \\
\hline QAM & Quadrature Amplitude Modulation \\
\hline
\end{tabular}




\begin{tabular}{|c|c|}
\hline QoS & Quality of Service \\
\hline QPSK & Quadrature Phase Shift Keying \\
\hline SATURN & Smart Antenna Technology in Universal bRoadband wireless Networks \\
\hline SIC & Serial Interference Cancellation \\
\hline SIMO & Single Input Multiple Output \\
\hline SM & Spatial Multiplexing \\
\hline SNIR & Signal to Noise plus Interference Ratio \\
\hline SNR & Signal to Noise Ratio \\
\hline SS & Spread Spectrum \\
\hline SUG & Scheduled User Group \\
\hline SVUG & SerVed User Group \\
\hline TDD & Time Division Duplex \\
\hline TDMA & Time Division Multiple Access \\
\hline TOA & Time Of Arrival \\
\hline TTI & Transmission Time Interval \\
\hline UGSV & User Group to be SerVed \\
\hline UMTS & Universal Mobile Telecommunications System \\
\hline UTRA & UMTS Terestial Radio Access \\
\hline VBLAST & Vertical Bell LAbs Space Time \\
\hline VBLAST AA-RRS & VBLAST Antenna-Assisted Round Robin Scheme \\
\hline WCDMA & Wideband-Code Division Multiple Access \\
\hline XOR & EXclusive OR \\
\hline$F$ & Zero Forcing \\
\hline
\end{tabular}




\section{Nomenclature}

Q Kronecker product

$(.)^{-1} \quad$ inversion

(.) ${ }^{H} \quad$ Hermitian (or conjugate) transpose

$(.)^{T} \quad$ transpose

$A_{D L} \quad$ random variable representing the shadow fading where $\log A_{D L}$

follows a Gaussian distribution

a input vector signal

â symbol vector recovered at the reception

$\hat{a}_{k_{i}} \quad$ estiamted symbol computed during the $k_{i}$ th repetition of the VBLAST algorithm

$a_{D L} \quad$ attenuation or path loss exponent

$\langle a, b\rangle \quad$ correlation coefficient between $a$ and $b$

$\underset{x}{\arg \max } f(x) \quad$ value of $x$ which maximizes the function $f(x)$

$\Gamma$

matrix of complex covariance coefficient used for the for

the generation of correlated channel coefficients

$\boldsymbol{\Gamma}_{s i n r} \quad$ post-detection SINR matrix

$\gamma_{i}(t) \quad$ instantaneous SIR (signal-to-interference ratio) at the time slot $t$ detected by user $i$

$\gamma_{k, n} \quad$ post-detection SINR for the channel corresponding to the

$n$th transmit antenna and the $k$ th user

$C \quad$ channel capacity

$C \quad$ symmetrical mapping matrix, that results from the standard Cholesky

factorization of the matrix $\boldsymbol{R}_{M I M O}=\boldsymbol{C} \boldsymbol{C}^{T}$

$\mathrm{C}_{M M S E} \quad M \times N$ matrix representing the MMSE detector

$\mathcal{D} \quad$ signal attenuation or path loss

$D_{X \rightarrow Y} \quad$ distance between $X$ and $Y$ and $\lambda$

$\mathbf{D}_{k, n}(t) \quad$ vector, whose elements are the transmit antennas that have been detected by user $k$ before the detection of transmit antenna $n$

$D R_{i, n}(t) \quad$ instantaneous data rate for the channel corresponding to the $n$th transmit antenna and the $i$ th user

$D R C_{i}(t) \quad$ instantaneous data rate experienced by user $i$ if it is served by the packet scheduler 


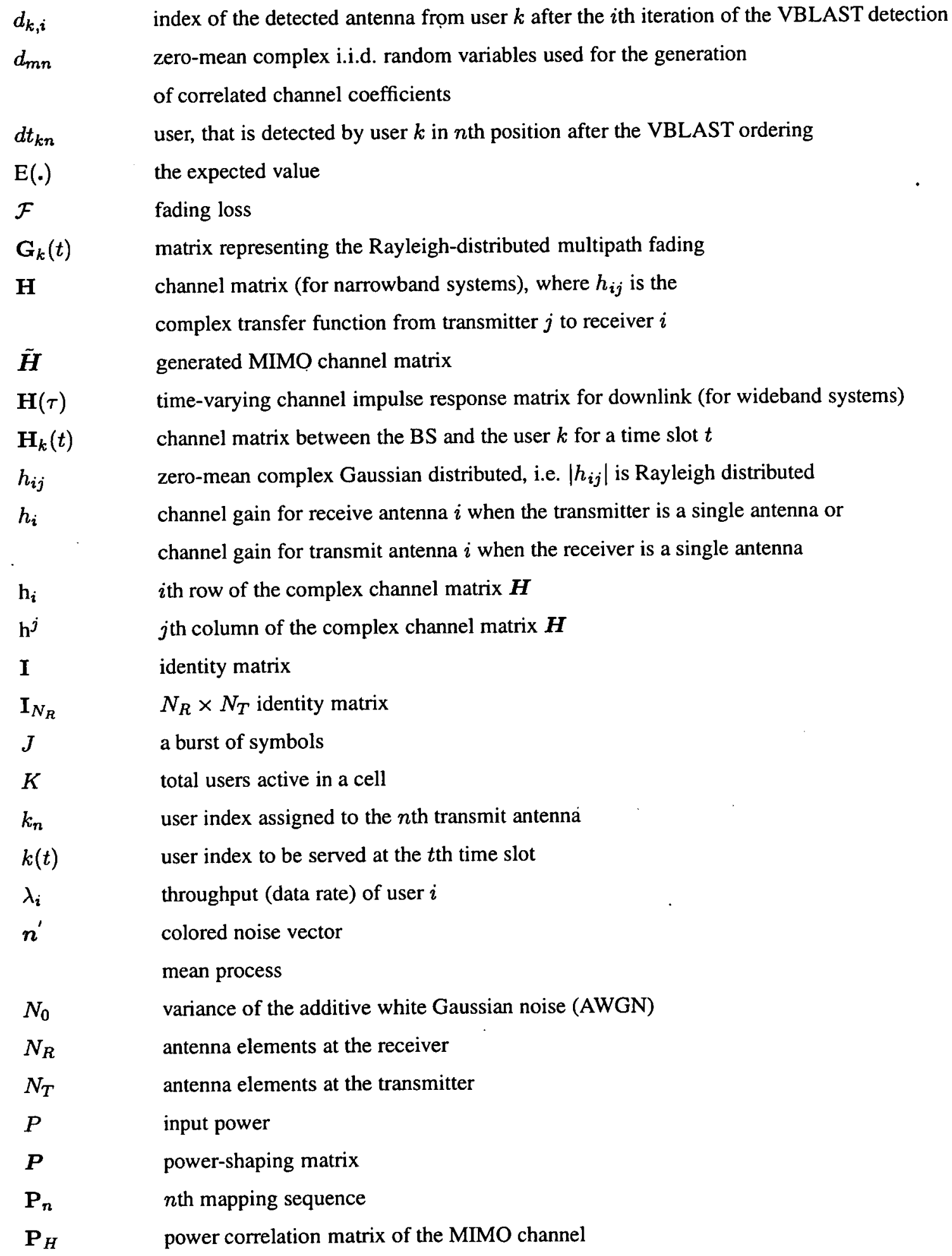




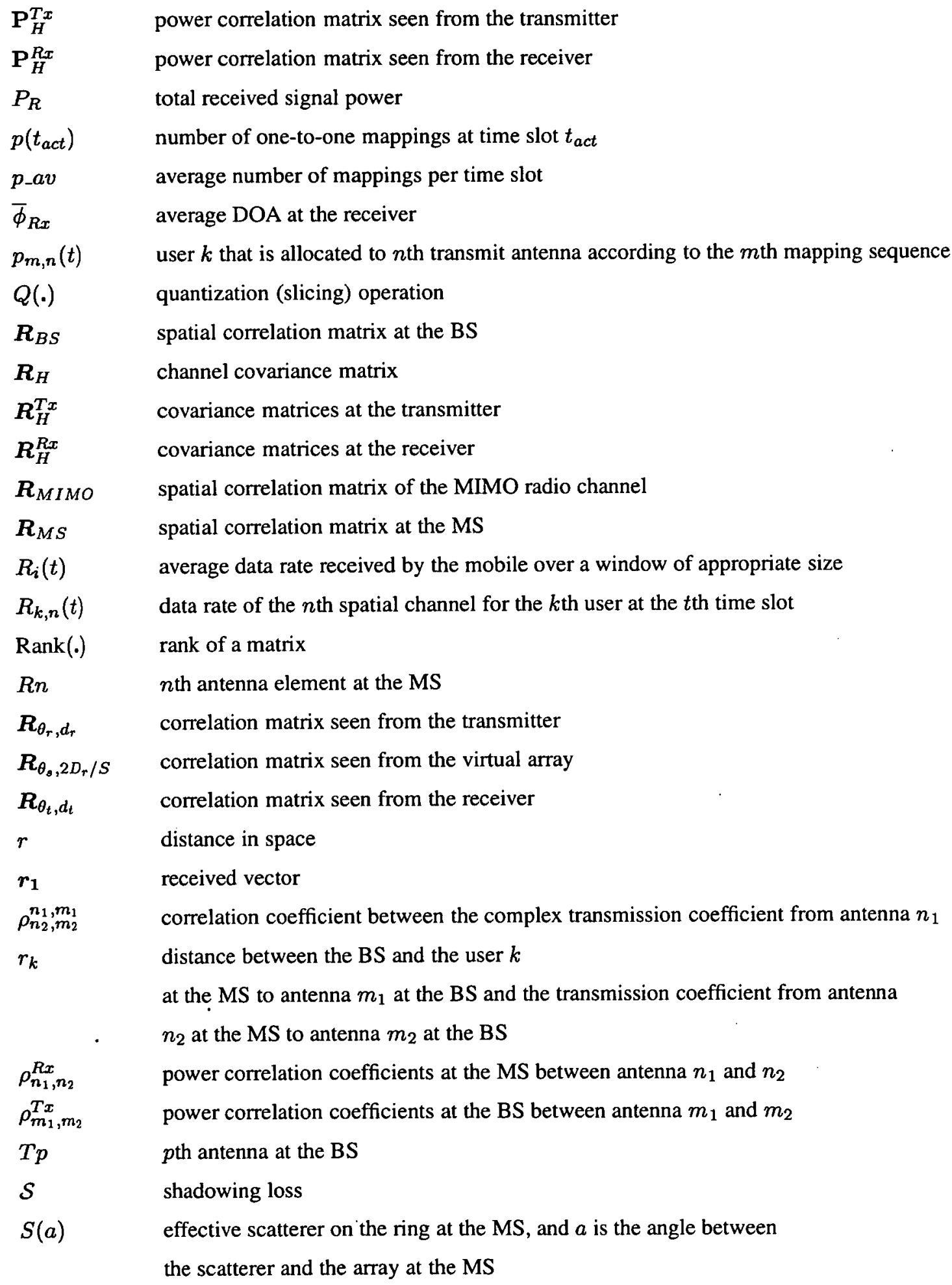




$\begin{array}{ll}\sigma_{h_{m n}} & \text { standard deviation of the channel coefficients } h_{m n} \\ S N R_{0} & \text { median SNR at the cell boundary } \\ S V U G & \text { set of user indices that have been already served } \\ T & \text { symbol period } \\ t & \text { time slot } \\ t_{a c t} & \text { time passed since the start of the last time period of }\left\lceil\frac{K}{N_{T}}\right\rceil \text { time slots } \\ U G S V & \text { set of user indices still to be served } \\ \operatorname{vec}(\cdot) & \text { vec-operator (vectorizes a matrix by stacking its columns) } \\ \mathbf{v} & \text { noise vector with components drawn from an i.i.d. wide-sense stationary zero } \\ \mathbf{W} & \text { nulling weight matrix for the } k \text { th user } \\ \mathbf{W}(t) & \text { steering diagonal matrix } \\ w_{k_{i}} & \text { nulling vector used for the computation of the } k_{i} \text { th decision statisic } y_{k_{i}} \\ \lceil x\rceil & \text { rounds the number x to the nearest integer greater than or equal to } x \\ y_{k_{i}} & \text { decision statistic computed during the } k_{i} \text { th repetition of the VBLAST algorithm } \\ \|z\| & \text { amplitude of the complex number } z\end{array}$




\section{Chapter 1 Introduction}

The origins of wireless communications go back to 1867 when James Clerk Maxwell predicted the existence of electro-magnetic waves (EMW) [1]. Around two decades later, in 1886, Heinrich Hertz provided an experimental evidence of their existence [2]. In the following decades many scientists continued to develop wireless communications in several directions. Nowadays digital wireless communication systems are part of our everyday life. In recent years we have witnessed a tremendous growth in the cellular networks and other wireless technologies. We watch digital (satellite) TV, talk on cellular phones or use wireless networking to access the internet. In particular, cellular networks are experiencing increasing demands for capacity and mobility. After the standardization of the $3 \mathrm{G}$ (third generation) cellular network - Universal Mobile Telecommunication System (UMTS) standard [3], researchers are refining concepts and technologies for wireless systems beyond 3G (e.g. fourth generation - 4G). These aim to provide the end user with a whole new range of wireless data communication services, like multimedia messaging, web browsing, online gaming, multimedia booking and reservation, streaming, etc [4].

The internet has become the largest network for exchange information worldwide. The volume of Internet Protocol (IP) based traffic has already exceeded that of circuit switched traffic in most fixed networks [5]. The provision of IP-based services in mobile communication networks has raised expectations of a exponentially growing traffic volume analogous to that already experienced over the Internet. In [6], the Universal Mobile Telecommunication System (UMTS) Forum predicts a considerable increase of the worldwide demand for wireless data services over the years to come of the present decade. Besides internet-like services, the addition of mobility to data communication enables new services not meaningful in a fixed network [7], e.g. location-based services. In this age of significant telecommunications competition, mobile network operators continuously seek new and innovative ways to create differentiation and increase profits. One of the best ways to do accomplish this is through the delivery of highly personalized services. One of the most powerful ways to personalize mobile services is based on location. Location based services (LBS) [8], could include services such as location based information. (e.g. list of restaurants within a certain proximity to the mobile user) or location 
based billing (after arrangement with the serving wireless carrier the user can establish personal zones such as a home zone or work zone). Since the majority of the wireless data services are asymmetrical, causing a heavier load in the downlink direction of the communication path, this work aims to investigates only the downlink performance.

Two of the key requirements in these systems are regarded as higher capacity and lower system cost [9]. A capacity increase in a cellular network can be achieved by increased bandwidth or higher spectral efficiency. While bandwidth is regulated, spectral efficiency can be modified in several ways. This work will focus on increasing spectral efficiency without degradation of the QoS. The quality of service (QoS) [10] experienced by the end user of a mobile communication system refers to the collective effect of service performance that determine the degree of satisfaction for this end user of the service. The QoS can be measured by means of certain parameters like bandwidth, delay, jitter, throughput, reliability and cost [10]. Typically, the user's data rate is one of key factors mostly for non real time services. The most crucial constraints on real time traffic are the packet transfer delay and user's data rate. Fundamentally, the more demanding these two constraints are, the lower the spectral efficiency that can be achieved by the mobile communication system.

Significant efforts have been made to improve the wireless spectrum efficiency in order to meet the future demand for high-data-rate (HDR) wireless communication. Some key techniques include: multi-user detection [11] [12] [13] that can increase spectral efficiency, receiver sensitivity, and the number of potential users, antenna arrays [14] [15] [16] [17] [18] [19] [20] [21] [22] [23] [24] [25] [26] [27] [28] that can minimize interference and improve spectral efficiency by exploiting spatial and multiuser diversity. Other techniques include, channel error control coding [29], power control [30], admission control [31], multiple access [32], OFDM (Orthogonal Frequency-Division Multiplexing) [33]. Also packet scheduling is an efficient transmission technique [34] [35] [36] [37] for providing service performance guarantees, such as throughput, delay, delay-jitter, fairness, and loss rate.

In this work we focus our investigation on packet scheduling strategies and multiple-input multiple-output (MIMO) antenna systems. Our main objective is to combine these two techniques in order to improve the system's efficiency and the QoS. The objectives of this work will be discussed in more detail in the next section. 


\subsection{Objectives of the thesis}

A third-generation wireless system faces a number of challenges. These include a complex time-varying multipath propagation environment, limited availability of radio spectrum, limited energy-storage capability of batteries in portable units, user demand for higher data rates, better voice quality, fewer dropped calls, enhanced in-building penetration and longer call times. Operators demand greater area coverage by base stations, increased subscriber capacity and lower infrastructure and operating costs. A number of different technologies have been used to meet such diverse requirements, including multiple antennas, packet scheduling, multiple access schemes, bandwidth-efficient source coding, and sophisticated signal-processing techniques. Cellular signal processing includes modulation and demodulation, channel coding and decoding, equalization, and diversity combining for fading channels (e.g. switched diversity or selective diversity).

Although a lot of research effort has been devoted to each of these technologies, the cooperation and combination of these techniques has not been investigated sufficiently yet. Consequently, there is a need for further research of this topic, to address the needs of a 3G and $4 \mathrm{G}$ communication systems. In order to be able to facilitate the evolution of the wireless communication market, $3 \mathrm{G}$ and $4 \mathrm{G}$ technology should be able to increase the system capacity not only for best service (effort) traffic. A $3 G$ system should also be able to meet the QoS demands of third generation mobile data services. The concept of QoS is a key factor in $3 \mathrm{G}$ technology evaluation because there exists a fundamental trade-off between the QoS provided to the end user and the achievable network throughput [38]. The main objective of this work is to investigate the system efficiency of the combination of different wireless techniques; as well as this existing trade-off between the QoS provided to the end user and the achievable network throughput by this technologies for different wireless scenarios.

In particular, special attention will be paid to the performance of MIMO arrays combined with a variety of scheduling schemes and detection methods. At first, a proper downlink channel model is selected, after a survey among a number of downlink MIMO channels. Then, a systematic comparison of a number of existing detection algorithms in various scenarios of interest is presented. This comparison gives an intuition about the relationship among the performance as well as other characteristics of the considered techniques, and helps the identification of the most suitable techniques. Next, we propose some new scheduling techniques that aim to combine the benefits of the previously examined detection techniques in a MIMO system and are based on popular packet scheduling schemes such as the round robin scheme [39] and the pro- 
portional fair scheme [36]. Simulation have been executed in order to examine the performance of these scheduling schemes in terms of system efficiency (cell capacity) and QoS (fairness among the users, time delay and outage capacity).

In this work we study only the downlink channel performance. This is due to the nature of the services that are planned to be offered by the third and future generations of mobile communication systems (e.g. browsing the Internet or downloading music and/or video files, shopping through the mobile phone etc., all of which require higher data rates on the downlink than the uplink). For this kind of services the downlink channel (the link from base station to mobile station) is expected to be more heavily loaded than the uplink (the link from mobile station to base station). Consequently, there is more need in for further research of the downlink.

\subsection{Outline of the Dissertation}

The remainder of this thesis is organized as follows:

Chapter 2: the objective of this chapter is to provide a survey of the resent developments in the field of wireless communication, focusing on some specific concepts such as multiple-input multiple-output (MIMO) antennas and packet scheduling. For this purpose, we investigate MIMO systems providing some basic information-theoretic results, and we expand these results for the case of multi-user channels. The chapter further aims in addressing the principles and techniques of packet scheduling in wireless networks. The basic characteristics of wireless communication, such as the attenuation effect caused by wireless propagation and the different transmission methods, are also presented.

Chapter 3: studies the physical channel performance in the case of MIMO systems. The objective of this chapter is to investigate the performance of a simple MIMO radio channel model, using some popular detection algorithms (such as ZF (Zero-Forcing equalizer), MMSE (Minimum Square Error Detector) and VBLAST (Vertical Bell LAbs Layered Space-Time Architecture)). For this purpose, we introduce the concept of spatial fading correlation and we present a variety of different stochastic and geometrically-based stochastic models, highlighting the common points and key differences between physical and non-physical MIMO channel models. Further, we chose the MIMO channel model that will be used throughout this work. The chapter finishes by investigating the performance of this model (in terms of bit error rate 
(BER) values), for different simulation setups. Specifically, simulation results, for different modulation schemes (BPSK, 16-QAM), detection techniques (VBLAST, MMSE), number of transmit and receive antennas and degree of spatial correlation, are presented.

Chapter 4: concentrates on the packet scheduling entity of MIMO multiuser wireless systems. The chapter investigates the downlink performance of various scheduling strategies based on Round Robin Scheme (RRS) and proposes some new packet scheduling algorithms (VBLAST AA-RRS, best user, AA MMSE fair and AA VBLAST fair). The new schemes aim to exploit multiuser diversity and multiple antennas in order to enhance the system performance in terms of cell throughput and outage capacity, and at the same time to follow the faimess rule imposed by the RRS scheme. The basic idea is to use multiple antennas to achieve a diversity effect from multiple users and at the same time enhance this diversity gain by using the VBLAST detection method. The performance of various scheduling algorithms will be discussed taking into account the concepts of fairness among the users and the time delay experienced by a user between two packet transmissions to the same user. For this purpose, simulations for different setups are carried out.

Chapter 5: concentrates on the proportional fair (PF) scheduling scheme. We show that PF can improve network throughput compared to RRS (Chapter 4), and at the same time can provide some form of faimess to all users. The objective of the chapter is to improve the proportional fair scheme. For this purpose we combine the PF scheme with MMSE or VBLAST detection techniques within a MIMO system, aiming to improve wireless resource efficiency (cell throughput) by exploiting time-varying channel conditions while at the same time controlling the level of faimess/QoS among users (time delay and outage capacity). Finally, the proposed scheduling schemes: AA-PF-MMSE single, AA-PF-MMSE multi, AA-PF-VBLAST single, AA-PF-VBLAST multi and AA-PF-VBLAST multi fair, are compared in terms of system efficiency and faimess among users, for different simulation setups.

Chapter 6: draws the the main conclusions of this Ph.D. investigation and discusses future research topics. 


\section{Chapter 2 System model - Related Work}

\subsection{Introduction}

The objective of this chapter is to provide a survey of the recent developments in the field of wireless communications, focusing on some specific concepts such as multiple-input multipleoutput (MIMO) antennas and packet scheduling. The chapter starts by introducing the concept of a wireless communication system. We discuss the basic characteristics of wireless communication and we concentrate our study on the attenuation effect caused by wireless propagation. In Section 2.2.2 we focus on multiple access methods for multiuser communications - FDMA (Frequency Division Multiple Access), TDMA (Time Division Multiple Access) and CDMA (Code Division Multiple Access). The high-data rate networks (HDR) are studied in Section 2.3. Section 2.3.1 presents the multiple-input multiple-output (MIMO) technology and in Section 2.3.2 we provide some basic information-theoretic results for MIMO systems (single user). These results are expanded for the case of multi-user channels in Section 2.3.3. In Section 2.4 we discuss the principles of packet scheduling in wireless networks and we present the three basic policies of scheduling. In the last Section 2.4 .2 we provide the actual methods (algorithms) that implement the scheduling policies described in Section 2.4 .

\subsection{Wireless Communication System}

In wireless communications the information to be sent is modulated onto a radio frequency (RF) signal which defines the carrier frequency. After modulation of the signal, the carrier signal is transmitted in the radio channel. The range of carrier frequencies useful for communications starts at a few thousand Hertz $(\mathrm{Hz})$ and goes up to several gigahertz. Therefore, the characteristics of the wireless channel are dependent on the radio propagation effects of the environment [40]. A typical outdoor wireless communication scenario is depicted in Figure 2.1 where the mobile station (MS) is communicating with a wireless base station (BS). The signal transmitted from the MS may reach the BS directly (line of sight) or through multipath 


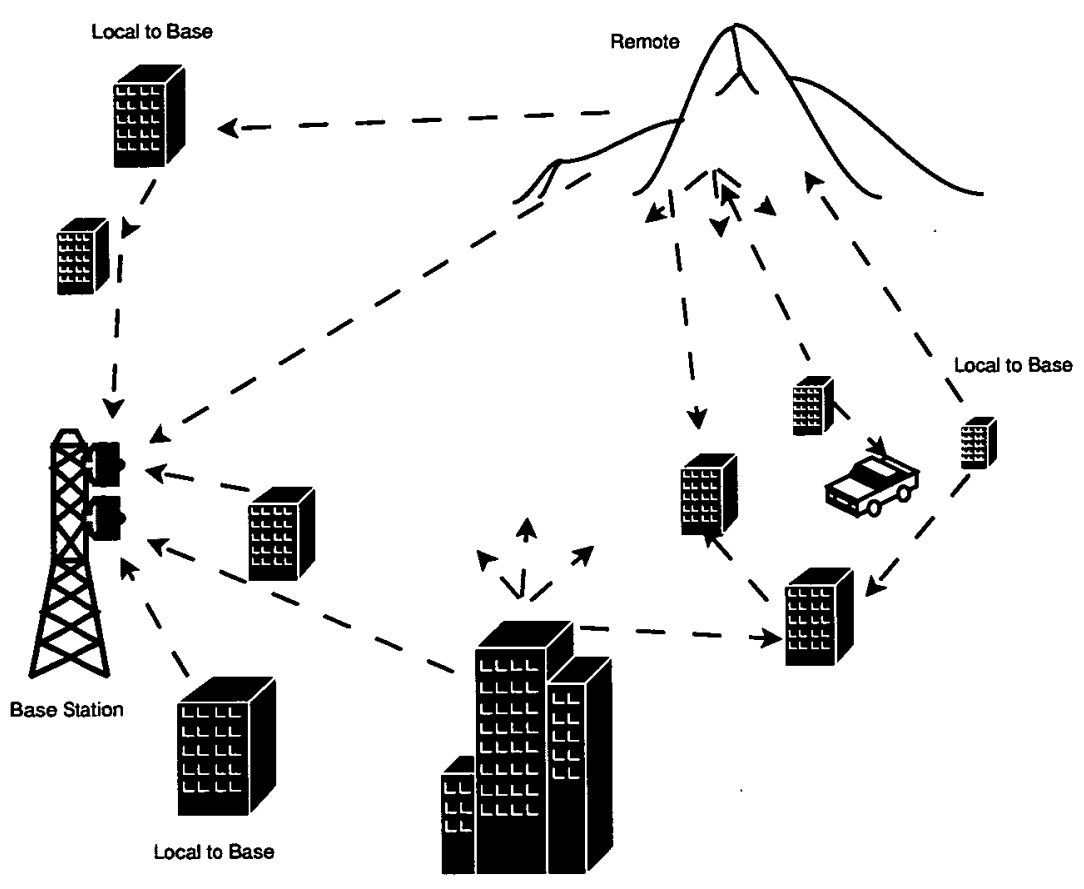

Figure 2.1: Radio Propagation Scenario

reflections on local scatterers (buildings, mountains, etc.). Because of that, the received signal is changed by multiple random attenuations and delays. Also, the movements of either the mobiles or the scatters can cause these random fluctuations to vary over time. Signal multipath occurs when the transmitted signal arrives at the receiver via multipath propagation paths at different delays. It generally results in intersymbol interference. Moreover, the signals arriving via different propagation paths may add destructively, resulting in signal fading [41]. The combined impacts of these phenomena makes the wireless environment challenging.

The attenuation incurred by wireless propagation can be decomposed in three main factors: a signal attenuation due to the distance between communicating nodes (path loss), attenuation effects due to absorption in local structures such as buildings (shadowing loss) and rapid signal fluctuations due to constructive and destructive interference of multiple reflected radio wave paths (fading loss) [42]. The signal attenuation or path loss $\mathcal{D}$ is due to the fact that, when radio waves transmitted from an omnidirectional antenna propagate through an environment, the fraction of the transmitted power intercepted by a receiver at a distance $r$ away from the receiver decreases with $r$. Empirically [40], [43] this attenuation $\mathcal{D}$ behaves as $\mathcal{D} \sim r^{a_{D L}}$. The exponent $a_{D L}$ takes values typically in the range of 3.5-4 [44] and captures environment effects 
such as ground reflections of the propagation waves. The time scale over which $\mathcal{D}$ varies depends on how the distance varies with time as determined by the mobility of the communication nodes. Typically path loss is the slowest varying among the attenuation effects.

The shadowing loss $\mathcal{S}$ is much more difficult to model, since it is due to absorption of the radio waves by scattering structures. It can be thought of as a random variable that takes a different value for each environment. Empirically, $\mathcal{S}$ is found to be well modeled by a lognormal distribution, i.e., the attenuation behaves as $\mathcal{S} \sim A_{D L}$, where $\log \left(A_{D L}\right)$ follows a Gaussian distribution [40]. In other words, the attenuation measured in decibels is Gaussian distributed. Furthermore, time variation occurs when the scattering environment changes, for example, when a mobile turns a comer on street. The time variation of the shadowing loss is quite difficult to model, and there exist few experimental studies. Qualitatively, we may state that shadowing loss varies with time faster than the path loss, but slower than the fading loss described below [42].

The fading loss $\mathcal{F}$ occurs due to the constructive and destructive interference of multiple reflected radio wave paths. Therefore, $\mathcal{F}$ varies when we sample the radio wave in different spatial locations. In a dense multipath environment, when there are enough scatterers, the complex amplitude is well modeled by a Gaussian distribution [40], [43]. If there is a line of sight path, the mean of the Gaussian distribution is nonzero leading to an attenuation (absolute value of the complex amplitude) which is Rician distributed [41] and, hence, termed Rician fading. When there is no line of sight, as would be the case in dense urban environments, we would have a complex amplitude which is zero-mean Gaussian distribution, leading to an attenuation which is Rayleigh distributed, and, hence, we have Rayleigh fading channels [41] [42].

In the case where we have multiple antennas, the relative attenuations between the antennas becomes an important issue. When the receive antennas are not far apart (of the order wavelengths of carrier frequency), the main variation arises due to the fading effects [45]. The relative response depends on the behavior of arrival directions of the reflected radio waves with respect to the receive antennas, which is characterized by the angular spread of the multiple paths [40]. In conclusion, the overall radio propagation attenuation is the combination of the phenomena described above $(\mathcal{D}, \mathcal{S}$ and $\mathcal{F}$ ) deteriorated by the time varying nature of the channel. Although in a near future, we will be surrounded by a numerous of options to set up an unwired connection over the radio interface, so far, the cellular approach is by far the most common wireless method to access data or to perform voice dialling. In the next section we present a brief description of the cellular network concept. 


\subsubsection{Cellular Radio System}

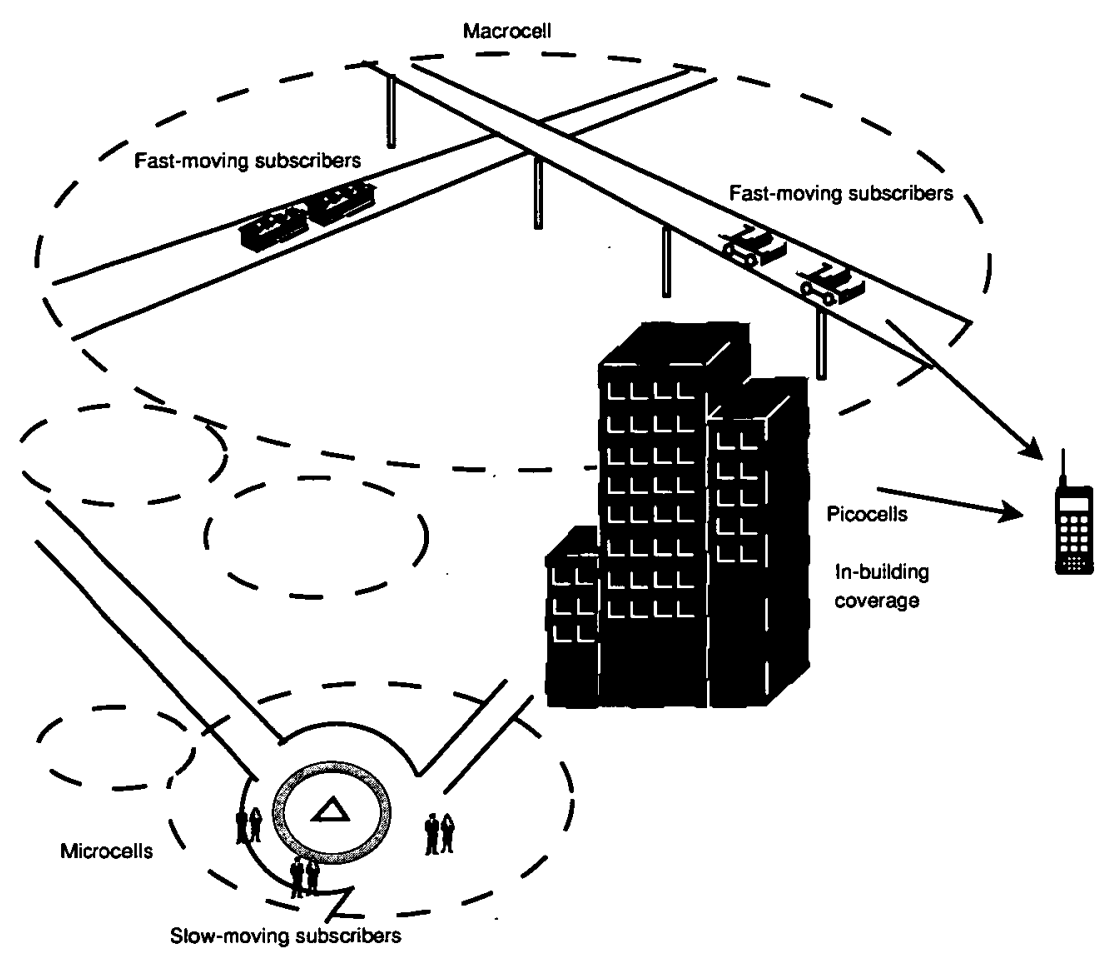

Figure 2.2: Cellular radio system

A cellular system [46] refers to communications systems that divide a geographic region into sections, called cells. The purpose of this division is to make the most use out of a limited number of transmission frequencies. Each connection, or conversation, requires its own dedicated frequency, and the total number of available frequencies is about 1,000 [47]. To support more than 1,000 simultaneous conversations, cellular systems allocate a set number of frequencies for each cell. Two cells can use the same frequency for different conversations so long as the cells are not adjacent to each other. A typical wireless communication scenario where the cellular radio approach can be used is depicted in Figure 2.2.

\subsubsection{Transmission and multiplexing}

In this section, we focus on multiple access methods for multiuser communications. A limited amount of bandwidth is allocated for wireless services. A wireless system is required to accommodate as many users as possible by effectively sharing the limited bandwidth. Therefore, 


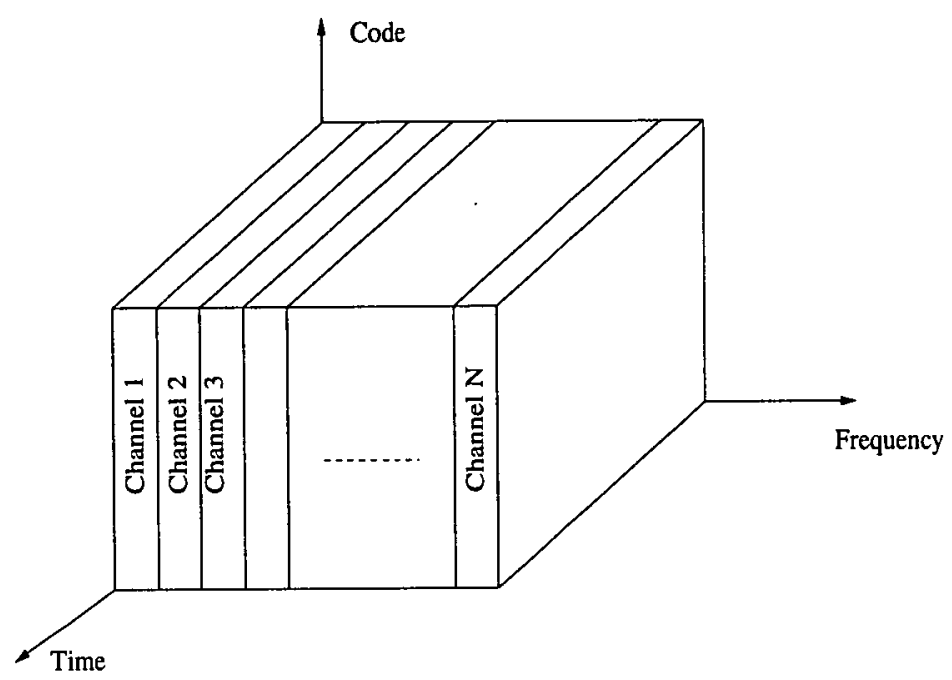

Figure 2.3: FDMA where different channels are assigned different frequency bands.

in the field of communications, the term multiple access could be defined as a means of allowing multiple users to simultaneously share the finite bandwidth with least possible degradation in the performance of the system. In general, there are several ways in which multiple users can send information through the communication channel to the receiver. The are three basic schemes (see Figures 2.3-2.4) [41]:

- Frequency division multiple access or FDMA, used in analog cellular, where calls are separated by frequency

- Time division multiple access or TDMA, used in digital cellular and PCS (personal communications services), where calls are separated by time

- Code division multiple access or CDMA, used mostly for PCS, where calls are separated by code

\subsubsection{Frequency Division Multiple Access - FDMA}

FDMA [40], [48] is one of the earliest multiple-access techniques. In this technique the frequency spectrum is divided into a number of logical channels. Each of these individual channels are assigned to individual users. Each user has a finite portion of bandwidth for permanent use 


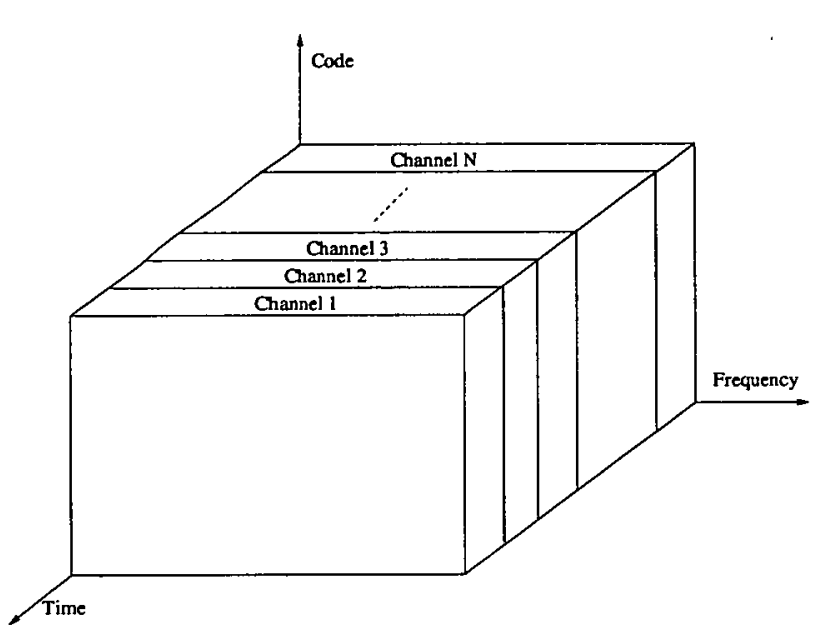

(a)

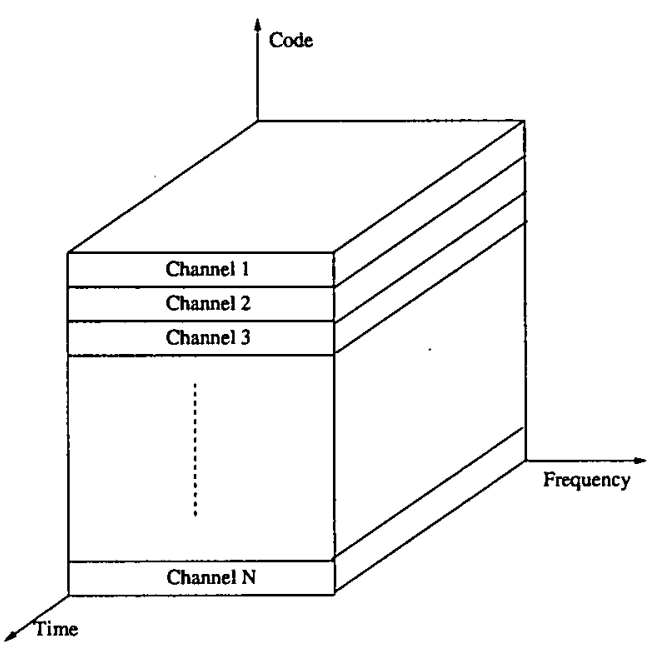

(b)

Figure 2.4: Multiple access schemes: (a)TDMA scheme where each channel occupies a cyclically repeating time slot, (b) Spread spectrum multiple access in which each channel is assigned a unique PN code which is orthogonal or approximately orthogonal to PN codes used by other users.

as illustrated in Figure 2.3. When a channel is not in use (not requested by any user) it becomes a wasted resource. The bandwidths of FDMA channels are relatively narrow (30Khz in AMPS-advanced mobile phone system) as each channel supports only one voice call per carrier. Therefore, FDMA is usually implemented in narrowband systems. Since the channels are allocated permanently to the users, fewer bits are needed for overhead purpose ( such as synchronization or timing control) compared to TDMA. FDMA requires tight RF filtering to minimize adjacent channel interference. After the assignment of a channel to a user, the base station and the mobile user transmit simultaneously and continuously. When continuous transmission is not required, bandwidth is wasted since it is not being utilized for a portion of the time. In wireless communications, FDMA achieves simultaneous transmission and reception by using frequency division duplexing (FDD). FDD provides two distinct bands of frequencies for every user. The forward band provides traffic from the base station to the mobile, and the reverse band provides traffic from the mobile to the base station. In order for both the transmitter and the receiver to operate at the same time. 


\subsubsection{Time Division Multiplex Access}

Time division multiplex access (TDMA) (Figure 2.4(a)) [40], [48] systems divide the bandwidth into time slots, and in each slot only one user is allowed to either transmit or receive. Each user is authorized to use a cyclically repeating time slot, so a channel may be thought of as a specific time slot that reoccurs every frame. TDMA systems use noncontinuous transmission, as users do not use the allocated bandwidth all the time. Global systems for mobile communications (GSM) [40] uses the TDMA technique. TDMA has the advantage that it is possible to allocate different numbers of time slots per frame to different users. As a result, bandwidth can be supplied on demand to different users by concatenating or assigning time slots based on priority. On the other hand, high synchronization overhead is required in TDMA systems because of burst transmissions. TDMA transmissions are slotted, and this requires the receivers to be synchronized for each data burst, because users share the bandwidth in the frequency domain. TDMA is almost always used alongside FDMA and FDD; the combination is referred to as FDMA/TDMA/FDD. This is the case in both GSM and IS-136 for example [40]. The exceptions to this rule include WCDMA-TDD which combines FDMA/CDMA/TDMA and TDD (time division duplexing) instead. TDD uses time to provide both forward link for transmission and reverse link for reception.

\subsubsection{Code Division Multiplex Access}

In CDMA (code division multiplex access) [40], [48], all the users occupy the same bandwidth, but they are all assigned separate codes, which differentiates them from each other as shown in Figure 2.4(b). In CDMA systems, the narrowband message signal is multiplied by a very large bandwidth signal called the spreading signal and this technique is called spread spectrum technique. In direct sequence spread spectrum (DS-SS), which is the most commonly used for CDMA, the spreading signal is multiplied by a pseudo random noise code (PRN code), which has noise-like properties. Each user has his own pseudorandom codeword which is ideally orthogonal to all the codewords of the other users. For detection of the information, the receiver needs to know the codeword used by the transmitter. For security reasons, each user operates independently with no knowledge of the other users (unlike TDMA, where time synchronization between the users is needed). Self-jamming is problem for CDMA. Self-jamming occurs when the the spreading codes used for different users are not exactly orthogonal. As a result, there is a significant contribution from other users to the receiver decision statistic in the de- 
spreading of a particular PRN code. Another important issue in CDMA is the near-far problem. The near-far problem occurs at the receiver when an undesired user has a high detected power compared to the power of the desired user. A technique for reducing the effects of the near-far problem is the employment of power control in the CDMA system. This ensures that all the signals within a cell arrive with the same power at the receiver. CDMA is depicted in Figure 2.4(b).

\subsection{High Data Rate wireless networks (HDR)}

In this section, we concentrate on the information-theoretic results that have led to recent developments in reliable high data rate (HDR) wireless networks. A good study on HDR (1xEV-DO (1xEVolution-Data Only)) is given by [49]. Techniques such as multiple antennas and packet scheduling have the potential to improve the performance of this system. In this section we concentrate on multiple arrays and in the next section we study packet scheduling.

One of the most significant recent development the last few years is the emergence of space time multiplexing techniques (multiple antennas), and some interesting information-theoretic results for multiple antennas are presented in this section. Another recent focus of interest is on the capacity of multiuser wireless networks. We also present some recent results on this topic. The capacity of fading channels was first studied in the 1960s ([50], Sec. 8.6]). At the same time, the foundations of network information theory were being laid out by characterizing the information-theoretic capacity of channels with many users ([51], Chapter 14]). Based on the fact that the wireless channel is an inherently shared medium, these results are of major concern in wireless systems domain. Although most of the problems in network information theory remain open, the multiple-access channel [51] is thoroughly analyzed. The multiple-access channel is of key interest because characterizes the communication from many terminals to a single terminal or base station.

The importance of diversity to communication in the presence of fading was recognized quite early [52]. Frequency, time, and space (multiple antennas) were identified as different means of providing independent realizations of the fading channel. We organize this survey of recent developments in spatial diversity as follows. In Section 2.3.1 we give a general description of a multiple-input multiple-output (MIMO) wireless system. Next, in Section 2.3.2 we review results in single-user multiple-antenna communication for fading wireless channels. The unique problems associated with multiple-user channels are treated in Section 2.3.3 where the concept 
of multiuser diversity is introduced.

\subsubsection{Multiple-Input Multiple-Output (MIMO) system}

In this section the necessary prerequisites for the following chapters of this thesis are presented. The general configuration for a communication link employing multiple transmitting and receiving antennas is depicted in Figure 2.5.

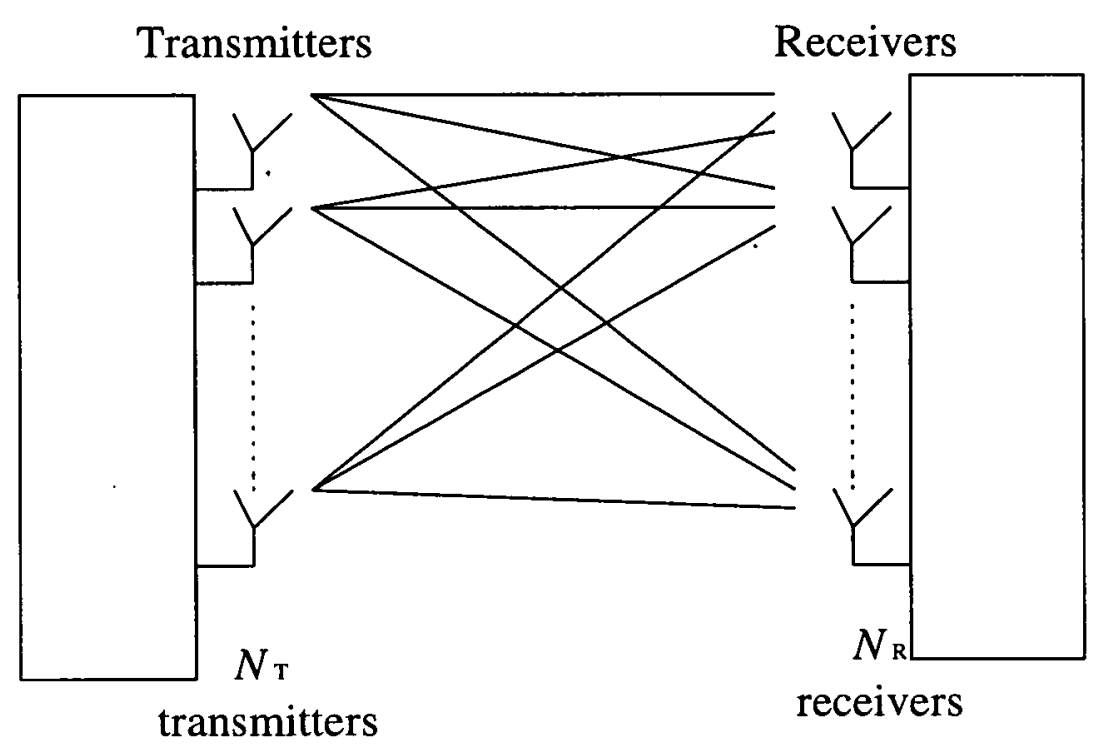

Figure 2.5: $M I M O$ wireless transmission system

The basic idea in MIMO transmission is that each user data stream is demultiplexed into $N_{T}$ sub- streams and each stream is then encoded and modulated to produce the signals to be transmitted over the wireless channel. A compressed digital source in the form of a binary data stream is fed to a simplified transmitting block encompassing the functions of error control coding and mapping to complex modulation symbols (quaternary phase-shift keying (QPSK), M-QAM, etc.). The latter produces several separate symbol streams. Each is then mapped onto one of the multiple transmit antennas. The mapping may include linear spatial weighting of the antenna elements or linear antenna space time precoding. After upward frequency conversion, filtering and amplification, the signals are launched into the wireless channel. At the receiver, the signals are captured by possibly multiple antennas and demodulation and demapping operations are performed to recover the message. The level of intelligence and complexity used in selecting the coding and antenna mapping algorithms can vary a great deal depending on the 
application. This determines the class and performance of the multi-antenna solution that is implemented [27].

Simple linear antenna array combining can offer a more reliable communications link in the presence of adverse propagation conditions such as multipath fading and interference [16] [27]. In the presence of random fading caused by multipath propagation, the probability of losing the signal vanishes exponentially with the number of decorrelated antenna elements being used [53]. This key concept is that of spatial diversity. The diversity order is defined by the number of decorrelated spatial branches available at the transmitter or receiver [53]. When combined together, antenna arrays are shown to improve the coverage range versus quality trade-off offered to the wireless user [54].

As mobile stations (MS) are gradually evolving to become sophisticated wireless Internet access devices rather than just pocket telephones, the stringent size and complexity constraints are becoming somewhat more relaxed. This makes multiple antenna elements transceivers a possibility at both sides of the link, even though pushing much of the processing and cost to the network's side (i.e., BTS (base transceiver station)) still makes engineering sense. Çlearly, in a MIMO link, the benefits of conventional antenna arrays (smart antennas) are retained since the optimization of the multi-antenna signals is carried out in a larger space, thus providing additional degrees of freedom [27]. In particular, MIMO systems can provide a joint transmitreceive diversity gain, as well as an array gain upon coherent combining of the antenna elements (assuming prior channel estimation) [16].

In fact, the advantages of MIMO are far more fundamental. The underlying mathematical nature of MIMO, where data is transmitted over a matrix rather than a vector channel, creates new and enormous opportunities beyond just the added diversity or array gain benefits. This was shown in [17], where the author demonstrates how one may under certain conditions transmit $\min \left(N_{T}, N_{R}\right)$ independent data streams simultaneously over the eigenmodes of a matrix channel created by transmit and receive antennas. Information theory can be used to demonstrate these gains rigorously (see Section 2.3.2). However, intuition is perhaps best given by a simple example of such a transmission algorithm over MIMO often referred to in the literature as VBLAST [55], [56], more generically called here spatial multiplexing. The VBLAST method is described extensively in the next chapter. The key aspects of VBLAST spatial processing of a received vector signal in detection of any substream are [17]: 1) interference nulling: interference from yet to be detected substreams is projected out, and 2) interference canceling: interference from already detected substreams is subtracted out. At the receiver, the received 
signals are separated and estimated. This occurs in the same way as three unknowns are resolved from a linear system of three equations. The separation is possible only if the equations are independent which can be interpreted by each antenna seeing a sufficiently different channel in which case the bit streams can be detected and merged together to yield the original high rate signal [53].

An analogy can be made with code-division multiple-access (CDMA) transmission in which multiple users sharing the same time/frequency channel are mixed upon transmission and recovered through their unique codes. The advantage of MIMO is that the unique signatures of input streams (users) are provided by nature in a close-to-orthogonal manner (depending however on the fading correlation) without frequency spreading, hence at no cost of spectrum efficiency. Unlike CDMA where user's signatures are quasi-orthogonal by design, the separability of the MIMO channel relies on the presence of rich multipath which is needed to make the channel spatially selective. Therefore, MIMO can be said to effectively exploit multipath [27].

In general [27], one will define the rank of the MIMO channel as the number of independent equations offered by the above mentioned linear system. It is also equal to the algebraic rank of the $N_{R} \times N_{T}$ channel matrix. Clearly, the rank is always both less than the number of transmit antennas and less than the number of receive antennas. In turn, following the linear algebra analogy, one expects that the number of independent signals that one may safely transmit through the MIMO system is at most equal to the rank [53].

\subsubsection{Information-Theoretic Results for Multiple-Antenna Channels}

The concept of channel capacity was introduced by Shannon in 1948 [57], where he showed that even in noisy channels, one can transmit information at positive rates with the error probability going to zero asymptotically in the coding block size. Perhaps the most famous illustration of this idea was the formula derived in [57] for the capacity $C$ of the additive white Gaussian noise (with variance $N_{0}$ ) channel with an input power constraint $P$, namely

$$
\left.C=\frac{1}{2} \log _{2}\left(1+\frac{P}{N_{0}}\right) \quad \text { (bits/channel use }\right)
$$

For scalar flat-fading channels, and a coherent receiver (where the receiver uses perfect channel state information), the capacity was shown to be [58] 


$$
C=\frac{1}{2} \mathbb{E}\left[\log _{2}\left(1+\frac{|h|^{2} P}{N_{0}}\right)\right] \quad \text { (bits/channel use) }
$$

where the expectation is taken over the fading channel $h$ and the channel is assumed to be stationary and ergodic. This is called the ergodic channel capacity [59]. This is the rate at which information can be transmitted if there is no feedback of the channel state from the receiver to the transmitter. If there is feedback available about the channel state, one can do slightly better through optimizing the allocation of transmitted power by waterfilling over the fading channel states [42]. The problem of studying the capacity of channels with causal transmitter side information was introduced by Shannon in [60], where a coding theorem for this problem was proved. When the transmitter has non-causal side-information the capacity was characterized by [61]. The understanding of achievable performance over scalar fading channels is covered in [62]. Also note that when we are dealing with complex channels (as is usual in communication with in-phase and quadrature-phase transmissions), the factor of $1 / 2$ in the equations above disappears [63]. From this point on, we assume complex channels are used.

In the presence of spatial diversity, the results above can be easily modified. The simplest case is when we have receive diversity (SIMO - single input multiple output system), i.e., when $N_{T}=1$ (transmitter antennas), for a given $N_{R}$ (receiver antennas) and flat-fading channels. Here it is [27] that the capacity is given by

$$
\left.C=\log _{2}\left(1+\frac{P}{N_{0}} \sum_{i=1}^{N_{R}}\left|h_{i}\right|^{2}\right)\right)
$$

where $h_{i}$ is the channel gain for receive antenna $i$. The crucial feature of equation (2.3) is that increasing the value of $N_{R}$ only yields in a logarithmic increase in average capacity. A similar expression when $N_{R}=1$ (MISO - multiple input single output system) can be derived [27]:

$$
\left.C=\log _{2}\left(1+\frac{1}{N_{T}} \frac{P}{N_{0}} \sum_{i=1}^{N_{T}}\left|h_{i}\right|^{2}\right)\right)
$$

where normalization by $N_{T}$ ensures a fixed total transmitter power. Again, note that capacity has a logarithmic relationship with $N_{T}$, similarly to SIMO case. Recent results in [17], [64], [28], [65] suggest significant advantages in using both transmitter and receiver spatial diversities i.e. $N_{T}, N_{R}>1$. In [28] the capacity of multiple-antenna fading channels was first 
established. In the flat-fading channel, the input vector $\boldsymbol{a}$ is independent of the fading process (as the transmitter does not have channel state information - CSI) and the Gaussian vector channel is memoryless. We also assume that the elements of $\boldsymbol{H}$ are i.i.d. Gaussian elements. In that case the ergodic (mean) capacity of the fading channel is [28]

$$
C=\mathbb{E}\left[\log \left(\left|I+\frac{P}{N_{T} N_{0}} H H^{H}\right|\right)\right]
$$

where (.) ${ }^{H}$ means transpose-conjugate and $\boldsymbol{H}$ is the $N_{R} \times N_{T}$ channel matrix. Note that the above result are based on $N_{T}$ equal power (EP) uncorrelated sources. Foschini [17] and Telatar [28] both demonstrated that the capacity in equation (2.5) grows linearly with $m=$ $\min \left(N_{T}, N_{R}\right)$ rather than logarithmically (as in equations (2.3) and (2.4)). This result can be understood as follows [27]: the determinant operator yields a product of $\min \left(N_{R}, N_{T}\right)$ nonzero eigenvalues of its (channel-dependent) matrix argument, each eigenvalue characterizing the SNR over a so-called channel eigenmode. For example, in Figure 2.6 the channel matrix $\boldsymbol{H}$ may offer $K$ parallel subchannels with different main gains, with $K \leq \min \left(N_{T}, N_{R}\right)$ [25]. The $k$ th eigenvalue can be interpreted as the power gain of the $k$ th subchannel [25]. An eigenmode corresponds to the transmission using a pair of right and left singular vectors of the channel matrix as transmit antenna and receive antenna weights, respectively. Thanks to the properties of the log function, the overall capacity is the sum of capacities of each of these modes, hence the effect of capacity multiplication. Note that the linear growth predicted by the theory coincides with the intuitive results presented in the previous section. Clearly, this growth is dependent on properties of the eigenvalues .

With the capacity defined by equation (2.5) as a random variable, the issue arises as to how best to characterize it. Two simple summaries are commonly used: the mean (or ergodic) capacity [28], [66], [67] and capacity outage [16], [68], [69]. A full description of the capacity would require the probability density function or equivalent. Some results are available in [70] but they are limited.

Some caution is necessary in interpreting the above equations. Capacity, as discussed here and in most MIMO work [16], [28], is based on a quasi-static analysis where the channel varies randomly from burst to burst. Within a burst the channel is assumed fixed and it is also assumed that sufficient bits are transmitted for the standard infinite time horizon of information theory to be meaningful. A second note is that in this section we have concentrated on single user MIMO 


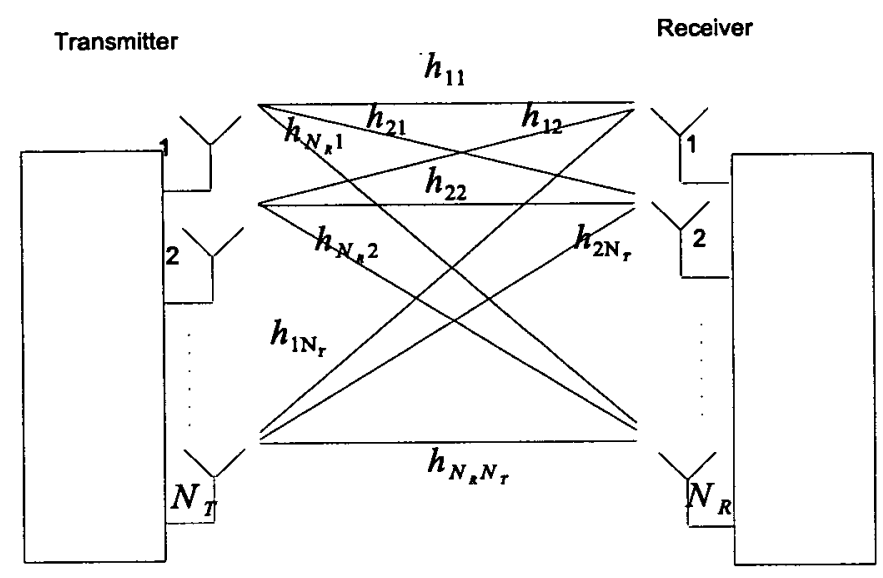

Figure 2.6: MIMO channel communication links

systems but many results also apply to multiuser systems with receive diversity (as we will see in the next sections). Finally, the linear capacity growth is only valid under certain channel conditions [27]. It was originally derived for the independent and identically distributed (i.i.d.) flat Rayleigh fading channel and does not hold true for all cases. For example, if large numbers of antennas are packed into small volumes, then the gains in may become highly correlated and the linear relationship will no longer hold due to the effects of antenna correlation [71], [72]. More generally, the effect of the channel model is critical.

\subsubsection{Multi-User Channels}

In wireless communications systems, it is often desirable to allow the users to send simultaneous information to the base station while receiving information from the base station. The inherent sharing nature of the wireless medium makes it possible. The study of multiuser communication techniques will be the object of this section. The general communication network consists of nodes trying to communicate with each other. In this study, we assume a specific network topology, the hierarchical communication topology [73] [42]. According to this topology one of the nodes obtains a special status as a base station or access point. The other nodes can only communicate to the base station. This type of topology is common in current commercial 
cellular wireless systems [73]. In the hierarchical topology, we distinguish between two types of problems: the first is the uplink channel where the nodes communicate to the access point (many-to-one communication or the multiple-access channel [51]) and the second is the downlink channel where the access point communicates to the nodes (one-to-many communication or the broadcast channel [51]). Given that there are multiple users, multiuser communication problems investigate the data rate region [42] that can be simultaneously achieved by the users. The characterization of this rate region is central to all network information theory problems. The rate region of the multiple-access fading channel is quite well understood both in the scalar case as well as the case with multiple antennas at the transmitter [62]. However, the rate region of the fading broadcast (downlink) channel is less well understood. For the single-antenna case, the rate region has been characterized in [74]. For the multiple-antenna broadcast channel, the rate region has not been completely characterized. Some recent progress in terms of characterizing the sum capacity of this channel has been made [75], [76], [77]. In this work we study the downlink channel throughput.

The coexistence of multiple users in a wireless channel gives rise to another form of diversity, known as multiuser diversity [78] [79]. The basic concept of diversity is: transmit the signal via several independent diversity branches to get independent signal replicas. Traditional forms of diversity include time, frequency and antennas. Multiuser diversity arises from independent fading channels across different users [79]. Multiuser diversity in a channel can be better understood when we look at the independent links from different users as pipes for information transfer [79]. The concept of multiuser diversity can be exploited by using packet scheduling techniques in a fading multiple-access channel [79]. The rate region for the uplink channel for a single cell was characterized in [80] where it was shown that in order to maximize the total information capacity, it is optimal to transmit only to the user with the best channel. For the scalar channel, the channel gain determines the best channel. The result in [80] when translated to rapidly fading channels results in a form of time-division multiple access (TDMA), where the users are not preassigned time slots, but are scheduled according to their respective channel conditions. If the users channels, are varying independently, then this strategy is a form of multiuser diversity where the diversity is viewed across users [42]. Here the multiuser diversity (which arises through independent channel realizations across users) can be hamessed using an appropriate scheduling strategy. A similar result also holds for the scalar fading broadcast channel [74], [37]. This idea is used in the downlink scheduling algorithm used in HDR [81] (high data rate $1 \mathrm{xEV}-\mathrm{DO}$ system), while ensuring faimess among users. Note that this requires 
feedback from the users to the base station about the channel conditions. The feedback could be just the received SNR. The discussion about scheduling will be continued in the next section.

\subsection{Packet Scheduling Principles and Strategies}

In wireline networks, scheduling policies play an important role in improving the system performance in terms of parameters such as throughput, delay, delay-jitter, fairness, and loss rate. Scheduling disciplines and associated performance problems have been widely studied in packet-switching networks [82], [83]. For a good survey of such algorithms, see [84]. However, scheduling schemes from the wireline domain do not transfer to wireless systems due to the unique characteristics of the wireless channels. Some of these characteristics are: (1) the wireless channels between the base station and the mobile users vary with time, and (2) the performance of the network depends on the channel and the signal processing techniques. Even if the resource is divided equally between different users, the network performance (e.g., throughput) could still be different from user to user due to channel attenuation.

As we have already discussed, radio propagation is generally characterized by three independent phenomena: path loss, log-normal shadowing and multipath-fading. Any of these phenomena vary with the movement of the mobile stations over time. On the other hand the interference that a user receives from other transmissions in the network is time-varying as well. The background noise also varies with the time constantly. The most common measures of the channel conditions include: SINR (signal to interference plus noise ratio), BER (bit error rate) and FER (frame error rate). Because the wireless channel is time-varying, users experience time-varying service quality (QoS). The voice users, who experience better channel conditions, could enjoy a better voice quality (higher achievable throughput). On the other hand in packet data service, better wireless channels (or higher SINR) could mean higher data rates available to the simultaneous users. In [36] it is demonstrated that when higher data throughput is allocated to the users with better links the cellular spectral efficiency (in terms of $\mathrm{b} / \mathrm{s} / \mathrm{Hz}$ ) can be significantly increased, although that happens at the expense of faimess among the active users [85].

Two fundamental goals of the packet scheduling are to maximize the network throughput while satisfying the QoS of the users providing fairness among users and satisfactory data rates. For the purpose of increasing the cell throughput, the scheduling scheme tries to exploit the the instantaneous channel variations by tracking the channel fluctuations and temporarily raising the priority of the users that experience the "best" channel conditions [86]. The wireless chan- 
nel conditions between the users and the base station tend to vary asynchronously (the users move randomly and the same can happen to the scatterers around them). This phenomenon combined with the time-shared nature of the downlink channel gives rise to a form of diversity, called multiuser or selection diversity, which can offer significant benefits for the spectral efficiency [87] [88]. Apart from enhancing the system throughput (spectral efficiency) the packet scheduler has to meet the QoS requirements imposed by the users. Hence, the effect of packet scheduling in the QoS will be taken into consideration in the performance evaluation in this work.

Several researchers have presented their work on the performance analysis of scheduling schemes that favor only the users with "best" channel conditions can lead to the starvation of the least favorable users (users with "bad" channels). There are several publications in the open literature that analyze the performance of packet scheduling algorithms that exploit the time-varying channel fluctuations in time shared mediums. Reference [89] presents the proportional fair (PF) algorithm, which provides a throughput leveling and an attractive trade-off between system throughput and faimess in a time-shared channel for delay tolerant data traffic. In [36], Jalali presents a performance evaluation of the proportional fair scheduler. Chapter 5 will thoroughly cover this scheduling scheme. In [85] [90], a performance comparison of several scheduling algorithms is presented. The results show a tendency of the schemes that achieve the highest the highest cell throughput to display the largest variations in throughput per user. More analytically, there are trade-offs among the throughput, delay, and fairness of an scheduling algorithm. Generally, those schemes that display the highest average sector throughputs also show the highest average delays per user, and the largest variations in the amount of resources allocated to each user. Schemes that display lower throughputs also provide lower average delays per user, and a fairer distribution of resources. In [91], six distinct prototype packet schedulers, including the proportional schedulers, have been considered and the inherent tradeoff among cell capacity and user fairness has been illustrated. In [92], an "opportunistic" transmission scheduling policy that exploits time-varying channel conditions and maximizes the system performance stochastically under a certain resource allocation constraint is presented. A method for providing QoS services over a shared high speed wireless data channel is presented in [93]. This approach is meant to enforce QoS constraints while still allowing for user diversity gains. In the present work and in the next chapters we intend to analyze the performance of a variety of scheduling algorithms with different degrees of fairness among users, some that exploit multiuser diversity and others that do not exploit multiuser diversity. As we mention above, the 
operation target of the scheduling algorithm is to balance the conflicting goals of maximizing throughput, while at the same time ensuring some degree of faimess of service to all users requesting it. The scheduling scheme determines the assignment of the radio resources among the simultaneous users. In [85] [90], the authors demonstrate that the scheduling algorithms that provide the highest cell throughput are proven to increase the unfairness between the favorable and the least favorable users. This phenomenon has an negative impact on the the degree of satisfaction among the users in the system. Motivated by this, in the next section we focus on the description of the most popular scheduling strategies of channel capacity allocation from the faimess point of view.

\subsubsection{Packet Scheduling Policies}

The purpose of the packet scheduler is to select the users to be served by the base station in the cell. The scheduler rules the allocation of the available resources to the active users of the cell. This resource allocation process depends on the scheduling strategy that has been assumed. In this work, the scheduling policies-strategies are divided into three basic categories:

1.C/ based: Scheduling policies based on a C/I (carrier-to-interference) strategy [94] favor users that experience the best instantaneous or average wireless channel conditions. This type of scheduler tends to maximize the system capacity at expenses of fairness among the simultaneous users. In practice, users with poor radio channel quality will be served when users with better radio propagation conditions do not have any more data to send. As a result, users with very bad channel conditions (at the edge of the cell) suffer a considerably poorer QoS than the remaining of the users in the cell, which in the case of a heavy loaded cell can cause these users to receive no data at all.

2.fair resources: This scheduling strategy [39] provides equal channel access chance to all users, which means that the radio resources (power, codes and allocation time) are equally distributed among the users in the cell. However, this results to lower throughput for the users at the edge of the cell compared to those close to the base station because the users with bad channels achieve lower data rates than the users with good channels. Also the the system throughput of this scheme is lower than the one achieved by $\mathrm{C} / \mathrm{I}$ based scheduling, since users with a low throughput get the same amount of resources as users with a high throughput [95].

3.fair throughput: This method [96] [97] aims at providing a fair throughput distribution among the users in the cell (regardless of their radio channel quality), while taking advantage of the 
short term fading variations of the radio channel. This resource allocation process is based on the max-min faimess. Analytically, a set of user throughputs $\{i\}$ is said to be max-min fair if it is feasible and the throughput of each user $\lambda_{i}$ can not be increased without maintaining feasibility and decreasing $\lambda_{j}$ for some other user $j$ for which $\lambda_{j}<\lambda_{i}$ [98]. Note, that a set of throughputs is feasible if the sum of all the users throughputs is lower or equal to the link capacity. The max-min faimess principle allows no increase in any $\lambda_{i}$, regardless of how large the increase is, if it is at the expense of the throughput decrease of any other user $j$ such that $\lambda_{j}<\lambda_{i}$, no matter how small decrease is. This means that all the users in the cell get the same amount of throughput no matter if they are close (good channel) or away (bad channels) from the base station. Another way to understand this strategy is to see it as an inverse form of $\mathrm{C} / \mathrm{I}$ scheduling, since users with low throughput are allocated larger amount of radio resources to achieve the same throughput. This policy tends to offer fair user throughput at the expense of lower system capacity.

Before moving to the next section, where we present the existing scheduling algorithms that realize the fairness strategies described here, we discuss some points concerning the relationship between the fairness, the QoS and the spectral efficiency. First, we have discussed above the tradeoff between the fairness among the users and the system capacity (spectral efficiency). Higher fairness means that the users with less good wireless channels tend to be allocated more time slots. As a result, a more fair user throughput distribution will lead to a lower system throughput [91]. Another interesting point is that the concept of fairness, although is a good indication for the QoS, does not represent an absolute criterion for the system's QoS. In particular, the fairness among the user does not refer to the absolute amount of throughput a user can receive, but to the relative distribution of the throughput or time among the users. Namely, even when the degree of fairness among the users is high (forced by the scheduling algorithm), the amount of radio resources the users may receive varies with the average or the temporal load. In practice, a heavy loaded cell will never keep its users satisfied no matter the degree of faimess among the users, because the end user is only interested in his absolute data rates, and not about his relative performance compared to the other users.

\subsubsection{Packet Scheduling Algorithms}

The present section introduces the existing algorithms that realize the fairness strategies described in section 2.4.1. The scheduling algorithms are classified in two main groups [97]: (I) 
"fast" packet scheduling methods that allocate the cell resources using recent channel quality measurements (i.e. executed on a TTI- Transmission Time Interval basis), thus allowing to track instantaneous variations of the user's supportable data rate. These methods can exploit the multiuser selection diversity, which can provide a significant capacity gain when the number of time multiplexed users is sufficient [99]. (II) "slow" scheduling methods that allocate the cell resources using average user's signal quality measurements (or that do not use any user's performance metric at all).

\subsubsection{Fast Scheduling Algorithms}

1. Maximum C/ (Max. CI): This scheduling algorithm [96] [100] always selects the users with the highest instantaneous carrier-to-interference (C/I) ratio. At the beginning of each TTI, the scheduler compares the $\mathrm{C} / \mathrm{I}$ levels of all active users and assigns the channel to the user with the highest $\mathrm{C} / \mathrm{I}$ value. Specifically:

$$
k(t)=\underset{i \in\{1,2, \cdots, K\}}{\arg \max } \gamma_{i}(t)
$$

where $k(t)$ denotes the user index to be served at the th time slot (TTI), $\gamma_{i}(t)$ is the instantaneous SIR (signal-to-interference ratio) at the time slot $t$ detected by user $i$. The maximum $\mathrm{C} / \mathrm{I}$ scheme represents the upper limit in terms of cell throughput because it can achieve the maximum multiuser diversity [100]. However, this is at the cost of lacking throughput fairness because users located far from the base station (poor channel conditions) are ultimately assigned less radio resources than the rest users.

2.proportional fair $(P F)$ : This algorithm was firstly described in [89] and further analyzed in [85], [96], [99], [101]. According to [36], the proportional fair scheduler serves the user with largest relative channel quality:

$$
k(t)=\underset{i \in\{1,2, \cdots, K\}}{\arg \max } \frac{D R C_{i}(t)}{R_{i}(t)}
$$

where $k(t)$ denotes the user index to be served at the $t$ th time slot, $D R C_{i}(t)$ is the instantaneous data rate experienced by user $i$ if it is served by the packet scheduler, and $R_{i}(t)$ is the average data rate (or average throughput) received by the mobile over a window of appropriate size. The detailed procedure for the computation of the user data rate $R_{i}(t)$ for the proportional fair 
algorithm is shown in Chapter 5. The proportional fair scheme selects a scheduled user based on the ratio of the instantaneous wireless channel conditions over the average channel condition with respect to each user. Namely, this scheme attempts to exploit the temporal fluctuations of the fast fading channel to increase the system throughput, while maintaining a certain level of fairness among all users. In [89], Holtzman demonstrated that the proportional fair algorithm asymptotically allocates the same amount of power and time resources to all users if their fast fading is iid (identically and independently distributed) and the rate $D R C_{i}(t)$ is linear with the instantaneous power. Note that this very last assumption does not hold in HSDPA (high-speed downlink packet access) due to the limitations of the AMC (adaptive modulation and coding) functionality [97]. The proportional fair scheme has the following interesting interesting property [97]. According to [36], the proportional fair policy provides the so-called proportional fairness solution. This fairness has been defined in [102]: a set of user data rates $\left\{\lambda_{i}\right\}$ is proportionally fair if it is feasible ( $\lambda_{i} \geq 0$ and $\sum_{\forall i} \lambda_{i} \leq C$, where $C$ system capacity) and if for any other feasible vector of data rates $\left\{\lambda_{i}^{*}\right\}$ the aggregate of proportional changes is zero or negative:

$$
\sum_{\forall i} \frac{\lambda_{i}^{*}-\lambda_{i}}{\lambda_{i}} \leq 0 \quad i=1, \cdots, K
$$

Proportional fairness is a more relaxed alternative of the min-max fairness (see Section 2.4.1), since it favours the poor channel quality users less emphatically. Note that min-max faimess gives an absolute priority to the smaller flow, in the sense that if $\lambda_{j}<\lambda_{i}$ then no increase in $\lambda_{i}$, no matter how large, can compensate for any decrease in $\lambda_{j}$, no matter how small.

\subsubsection{Slow Scheduling Algorithms}

1. Average C/I (Avg. CI): This scheduling scheme [96] in every TTI selects the user with largest average $\mathrm{C} / \mathrm{I}$.

2. Round robin scheme (RRS): RRS packet scheduler [39], selects the users in a pre-selected order (randomly created) regardless of the user channel quality conditions. An implementation of RRS scheme is to index the active users and then serve them in turns, from the lowest indexed user to the highest. RRS clearly belongs to the fair resources strategy described in the previous section. This scheme is very popular due to its simplicity and the fact that guarantees fair 
distribution of the radio resources among the users in the cell. The RRS scheme is described analytically in section 4.3.1.

The slow scheduling methods have a lower degree of complexity than fast scheduling ones. In particular, the fast scheduling schemes, in order to make the scheduling decisions, require information of channel condition measurements for all the users in the cell.

\subsection{Conclusions}

In this chapter we presented the literature background for the key components of a wireless communication system. We focused our study on multiple antennas (MIMO) and packet scheduling technologies. The key conclusions, that will be in the center of interest in the next chapters are summarized in the following points:

- the system capacity of a SIMO (receive diversity) and a MISO (transmit diversity) system increases logarithmically with the number of receive or transmit antennas respectively (see equations (2.3) and (2.4)).

- the system capacity of a MIMO system grows linearly with $m=\min \left(N_{T}, N_{R}\right)$ rather than logarithmically (as in case of SIMO or MISO arrays).

- literature results on packet scheduling indicate a tendency of scheduling schemes to show trade-offs among the throughput, delay, and fairness. Generally, those schemes that display the highest average cell throughputs also show the highest average delays per user, and the largest variations in the amount of resources allocated to each user. Schemes that display lower throughputs also provide lower average delays per user, and a fairer distribution of resources.

- we have considered three packet scheduling policies: (I) $\mathrm{C} / \mathrm{I}$ based, where the scheduler tends to maximizes the cell throughput at expenses of fairness among the simultaneous users by selecting the users with the best channel quality, (II) fair resources, where the scheduler provides equal channel access chance to all users, by distributing equal amounts of radio resources to all users in the cell, and (III) fair throughput, where the scheduler provides a fair throughput distribution among the users in the cell (regardless of their radio channel quality), while taking advantage of the temporal fading variations of the radio channel. 
The above key points show the potential gain of using multiple antennas in the system throughput and the role of packet scheduling in the trade-off between spectral efficiency and QoS in a wireless communication system. The next chapter, will aim to exploit the benefits offered by multiple antennas and packed scheduling to improve the system performance. 


\section{Chapter 3 \\ Physical Channel Performance}

\subsection{Introduction}

The last years, the employment of multiple antennas for wireless cellular networks has received much attention. Exploiting antenna arrays at both the transmitter and receiver allows the Shannon capacity of wireless channels to be increased significantly [27]. Multiple-input multiple-output (MIMO) systems offer large theoretical information capacities, realizing high throughput performance, especially in dense multipath environments (such as indoor scenarios). They can be a hopeful solution for high data rate broadband wireless local area networks (WLAN). However, the evolution of such systems, requires a precise characterization and modeling of the underlying wireless channel. In literature we can find a variety of different stochastic or geometrically-based stochastic MIMO models [26] [103] [104] [105]. Large data capacities can be obtained via the potential decorrelation in the MIMO radio channel, which can be exploited to create many parallel spatial subchannels. However the potential capacity gain is highly dependent on the multipath richness in the radio channel and the antenna array configurations. A fully correlated MIMO channel only offers one spatial channel, while a completely decorrelated channel offers multiple spatial channels [104]. Although in real wireless scenarios we would expect a partially correlated channel, much of the recent research work has focused upon either fully correlated or fully decorrelated wireless channels [26] [28]. The motivation for this chapter is to investigate the performance of a simple MIMO radio channel model, which can be used for link level simulations, using some popular detection algorithms. Some authors [106] have approached the problem from a geometrically-based perspective. A mathematical framework for a simple stochastic wideband MIMO channel was presented in [104]. In this chapter we use a simplified model first presented in [107]. One of the main strengths of the MIMO stochastic model is that it relies on a small set of parameters to fully characterize the communication scenario; the power gain of the MIMO channel matrix, two correlation matrices describing the correlation properties at both ends of the transmission links, and the associated Doppler spectrum of the channel paths [107]. The system model assumes $N_{T}$ transmitting antennas and $N_{R}$ receiving antennas $N_{T} \leq N_{R}$. Simulations are carried out 
for two modulation schemes (BPSK and 16-QAM). Multistream detection is implemented at the receiver. The minimum mean squared error detector (MMSE) [108] [109] is compared with its V-BLAST (Vertical Bell Laboratories Layered Space-Time) [110] version. Comparisons are made using bit error ratio (BER) versus signal-to-noise ratio (SNR) simulation. The effect of error propagation in the BLAST scheme is also investigated.

This chapter is organized as follows. In Section 3.2, we give a general description of the MIMO communication system. In Section 3.3, we discuss state of the art MIMO channel models, highlighting the common points and key differences between physical and non-physical MIMO channel models. Finally, we chose the MIMO channel model that will be used throughout this work. In Sections 3.4 and 3.5 we describe analytically our MIMO stochastic channel model. Next, we take a look at the receiver in Section 3.6, and three basic detection methods are presented : ZF (zero-forcing equalizer), MMSE (minimum square error detector), VBLAST (Vertical Bell LAbs Layered Space-Time Architecture). Bit error rate (BER) simulation results are presented in Section 3.7. Finally, the conclusions are given in Section 3.8.

\subsection{System description}

Consider the MIMO setup pictured in Figure 3.1 with $N_{T}$ antennas at the transmitter and $N_{R}$ antennas at the receiver. A single data stream is demultiplexed into $N_{T}$ substreams, and each substream is then encoded into symbols and fed to its respective transmitter.

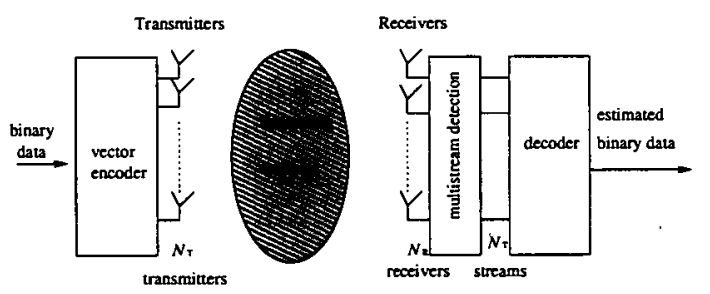

Figure 3.1: High-level view of a MIMO wireless communication system.

Let $T$ seconds be the symbol period. Transmitter antennas 1 to $N_{T}$ operate on the same radio frequency (RF) at a symbol rate $1 / T$ symbols/sec, with synchronized symbol timing. The collection of transmitters comprises, in effect, a vector-valued transmitter, where components of each transmitted $N_{T}$-vector are symbols drawn from a BPSK or QAM constellation. We also make the following assumptions: 1) narrowband operation, 2) all $N_{T}$ constellation are the same size, and 3) the transmitter does not know the channel realization, but the receiver learns it. In this chapter the same constellation is used for each substream, and transmissions are 
organized into a burst of $J$ symbols. The power launched by each transmitter is proportional to $1 / N_{T}$ so that the total radiated power is constant and independent of $N_{T}$. In V-BLAST, the vector encoding process is simply a serial-to-parallel operation followed by independent bitto-symbol mapping of each substream. For the remainder of this thesis, we will assume for simplicity that the substreams comprise uncoded, independent data symbols. Receivers 1 to $N_{R}$ are, individually, conventional BPSK or QAM receivers. These receivers also operate on the same radio frequency, each receiving the signals radiated from all $N_{T}$ transmit antennas. In this chapter each symbol to be transmitted is sent to exactly one transmitting antenna and the receiver is symbol-synchronous. Letting $a=\left(a_{1}, a_{2}, \ldots, a_{N_{T}}\right)^{T}$ denote the vector of transmit symbols, then the corresponding received $N_{R}$-vector is

$$
\boldsymbol{r}_{1}=\boldsymbol{H a}+\boldsymbol{v}
$$

where $v$ is a noise vector with components drawn from an i.i.d. (independent identicallydistributed random variables) wide-sense stationary zero mean process with variance $N_{0} . H$ is the $N_{R} \times N_{T}$ matrix channel transfer function, where $h_{i j}$ is the (complex) transfer function from transmitter $j$ to receiver $i$, and $N_{T} \leq N_{R}$. To keep the channel model simple, it is assumed that $h_{i j}$ is a zero-mean complex Gaussian distributed, i.e. $\left|h_{i j}\right|$ is Rayleigh distributed. We take the viewpoint that the channel time variation is quasi-stationary and therefore negligible over the $J$ symbol periods comprising a burst, and that the channel is estimated accurately, e.g. by use of a training sequence embedded in each burst. Thus, for brevity we will not make the distinction between $\boldsymbol{H}$ and its estimate. Note, that equation (3.1) implies frequency flat fading channel. In the following Section 3.2.1, a more detailed presentation of the MIMO Transmitter will be given. Then, in Section 3.3, some recently published research on MIMO channel modeling is reviewed.

\subsubsection{Transmitter Structure}

Consider the multiantenna transmitter diagram in Figure 3.2. A compressed digital source in the form of binary data stream is fed to a demultiplexer block. The data stream is demultiplexed among the $M$ transmitting antennas, each of which conveys a distinct substream. Each substream undergoes error control coding. The resulting binary sequences are fed to a QAM or BPSK modulator, which produces the symbols to be transmitted. Throughout this work the 
same constellation is used for each substream, and transmissions are organized into a burst of $J$ symbols.

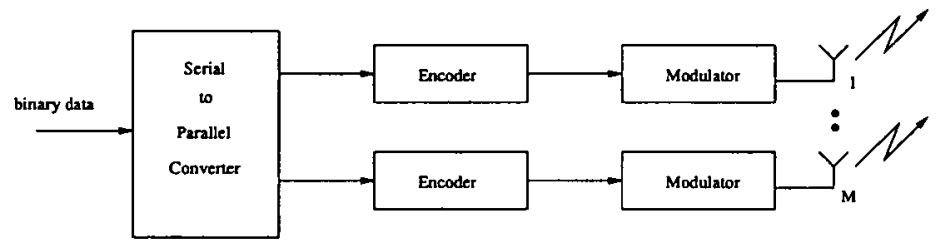

Figure 3.2: Diagram of MIMO wireless transmitter. The transmitter is equipped with $M$ antenna elements. Coding, modulation, and mapping of the signals onto the the antennas is realized separately.

\subsection{MIMO Channel Models}

Precise modeling of the MIMO channel impulse response matrix $\boldsymbol{H}(\tau)$ (for wideband systems) or MIMO channel matrix $\boldsymbol{H}$ (for narrowband systems) is essential for theoretical analysis, design and simulation of MIMO systems, therefore plenty of research work in modeling of MIMO channel has been conducted in the last few years. Different statistical and deterministic MIMO channel models have been presented. In [19] a deterministic MIMO channel model has been developed, where a wireless system engineering (WiSE) ray-tracing simulator has been employed to construct random instances of the MIMO channel matrix for indoor wireless environment. Concerning the domain of statistical MIMO channel modeling, many models have been developed, and these models can be categorized in different ways.

1. Non-physical models vs. physical models: the non-physical models [111] [112] are constructed using the first and second order moments of MIMO radio channels. These models are referring to scenarios with Rayleigh fading, since a zero-mean complex Gaussian process (channel) is completely specified by its first and second order moments [113]. On the other side, the physical models [114] [23] create a realistic geometric multipath propagation model from which the spatial correlation coefficients are derived as a sum of the contributions from different rays. The distribution of the scatterers is determined by some important physical parameters, such as AOA (angle of arrival), AOD (angle of departure) and TOA (time of arrival). The statistical data of these parameters can either be received from measurements [20] [115] or from some popular distributions with a simple geometrical scenario [114] [21] [116]. 
2. Wideband models vs. narrowband models: the radio channels can be split into wideband and narrowband models by taking into account the coherence bandwidth of the channel. If the channel coherence bandwidth is small in comparison to the bandwidth of the system, the channel is said to be frequency selective. If the channel coherence bandwidth is large in comparison to the bandwidth of the system, the channel is said to be frequencynonselective [41]. The wideband channel models [104] [111] [115] [117] consider the wireless channel as frequency selective, which means that the system's spectral components are affected differently (independently) over the system's frequency domain. On the contrary, the narrowband channel models [114] [116] [118] [24] [23] assume that the channel has frequency flat fading (frequency-nonselective) and therefore the channel has the same channel response over the system bandwidth.

3. Measurements vs. scatterer models: there are two ways to extract the basic characteristics of the MIMO radio. Either using field measurements results and the channel is modeled to have similar propagation characteristics, or by constructing a realistic propagation (scatterer) model that aims to emulate the channel characteristics. Models using the first approach (measurements) are presented in [111] [20] [117] [118]. Examples based on the propagation models are developed in [114] [23].

In this work, we divide the MIMO channel models using the first categorization approach. In particular we separate the models in two categories the physical and non-physical MIMO channel models. A detailed description of the model we have adopted will be given in Section 3.4. In the following sections, some basic statistical MIMO channel models are reviewed. Section 3.3.1, presents some non-physical MIMO channel models and Section 3.3.2, presents some physical MIMO channel models.

\subsubsection{Non-physical MIMO Channel Models}

\subsubsection{IST METRA Project}

METRA [119] (multi element transmit receive antennas) project is a research project partially sponsored by the European commission under the information society technologies programme (IST). The METRA project performed multiple-input and multiple-output (MIMO) matrix radio channel measurement to clarify the characteristics of the MIMO channel matrix in various mobile communication scenarios. Specifically, channel data were collected in two types of 
environments: picocell and microcell. Here the term picocell and microcell refer to indoor-toindoor and indoor-to-outdoor environment, respectively. For each environment several mobile station locations are selected to provide a set of measurements where both LOS (line-of-sight) and NLOS (non line-of-sight) scenarios are present. A stochastic MIMO channel model was introduced based on the power correlation matrix of the MIMO radio channel [104] [111] [107]. It was claimed in [104] that it can be shown theoretically that the spatial cross correlation coefficient can be expressed as the product of the spatial correlation at the transmitter and receiver:

$$
\rho_{n_{2}, m_{2}}^{n_{1}, m_{1}}=<\left|h_{n_{1}, m_{1}}\right|^{2},\left|h_{n_{2}, m_{2}}\right|^{2}>=\rho_{m_{1}, m_{2}}^{T x} \rho_{n_{1}, n_{2}}^{R x}
$$

where $h_{i, j}$ is the complex channel coefficient between the transmit antenna $j$ and the receive antenna $i$, and $\rho_{m_{1}, m_{2}}^{T x}, \rho_{n_{1}, n_{2}}^{R x}$ denote the power correlation coefficients at the transmitter and receiver respectively. In matrix form, equation (3.2) can be written as

$$
\boldsymbol{P}_{H}=\boldsymbol{P}_{H}^{T x} \otimes \boldsymbol{P}_{H}^{R x}
$$

where $\boldsymbol{P}_{H}$ is the power correlation matrix of the MIMO channel, $\boldsymbol{P}_{H}^{T x}$ and $\boldsymbol{P}_{H}^{R x}$ are the power correlation matrices seen from the transmitter and the receiver respectively.

Notice that the proposed model only reproduces the correlation metrics and fast fading characteristics of the radio channel, while the phase derivative across the antenna arrays is not necessarily reflected correctly in the model (as long as the power correlation coefficients do not carry phase information). In [104], it was proposed to multiply a steering diagonal matrix $W\left(\bar{\phi}_{R x}\right)$ after the convolution between the MIMO channel impulse response and the transmitted signal, therefore the received signal can be expressed as

$$
r_{1}=\boldsymbol{W}\left(\bar{\phi}_{R x}\right) \boldsymbol{H a}+\boldsymbol{v}
$$

where the diagonal elements of the diagonal matrix $W\left(\bar{\phi}_{R x}\right)$ describe the average phase shift information relative to the first receive antenna element and $\bar{\phi}_{R x}$ is the average direction of arrival (DoA) at the receiver. 


\subsubsection{IST SATURN Project}

An initial analysis of MIMO channel measurements taken in indoor environments at $5.2 \mathrm{GHz}$ is presented in [120]. The MIMO measurements were contributed by the EU IST SATURN (Smart Antenna Technology in Universal bRoadband wireless Networks) project. In [118], the above measurement results have been used to study the channel characteristics of MIMO indoor systems at $5.2 \mathrm{GHz}$ and a statistical narrowband model for the NLOS indoor MIMO channel based on the first and second order moments has been presented. A similar model has been developed in [107]. In [118], after analyzing the measurement results, it was concluded that the channel coefficients for these measured NLOS indoor MIMO scenarios appear to be zero-mean complex Gaussian. Moreover, it was shown the channel covariance matrix can be well approximated by the Kronecker product of the covariance matrices at the transmitter and receiver side respectively. This can be expressed as:

$$
\boldsymbol{R}_{H}=\boldsymbol{R}_{H}^{T x} \otimes \boldsymbol{R}_{H}^{R x}
$$

where $\boldsymbol{R}_{H}$ is the channel covariance matrix, $\boldsymbol{R}_{H}^{T x}$ and $\boldsymbol{R}_{H}^{R x}$ are the covariance matrices at the transmitter and the receiver respectively. The transmitter, receiver and channel covariance matrices are defined as:

$$
\begin{gathered}
\boldsymbol{R}_{H}^{T x}=E\left[\left(\mathrm{~h}_{i}^{H} \mathrm{~h}_{i}\right)^{T}\right], \quad \text { for } i=1, \cdots, N_{R} \\
\boldsymbol{R}_{H}^{R x}=E\left[\left(\mathrm{~h}^{j} \mathrm{~h}^{j^{H}}\right)\right], \quad \text { for } j=1, \cdots, N_{T} . \\
\boldsymbol{R}_{H}=E\left[\operatorname{vec}(\mathrm{H}) \operatorname{vec}^{H}(\mathrm{H})\right]
\end{gathered}
$$

where $\mathrm{h}_{i}$ is the $i$ th row of the complex channel matrix $\boldsymbol{H}$ and $\mathrm{h}^{j}$ is the $j$ th column of $\boldsymbol{H}$. Where, $(.)^{T}$ denotes transpose, $(.)^{H}$ Hermitian (or conjugate) transpose, vec(.) is the vecoperator (vectorizes a matrix by stacking its columns) and $\mathrm{E}($.$) denotes the expected value,$ which averages over all measured MIMO channel realizations for a specific transmitter and receiver pair. Notice the similarity between the equations (3.3) and (3.5). In 3.5, instead of the 
power correlation matrix the channel covariance matrix is used, as a result (3.5) provides the phase information of the MIMO radio channel. In Section 3.4, we will revisit this model in detail.

\subsubsection{Physical MIMO Channel Models}

\subsubsection{One-ring and Two-ring Models}

In [114], the narrowband 'one-ring' and 'two-ring' models were developed. The 'one-ring' model is appropriate in the fixed wireless communication context, where the base station (BS) is usually elevated and unobstructed by local scatterers, and the subscriber unit (SU) is often surrounded by local scatterers. The corresponding scenario is pictured in Figure 3.3. $T p$ is

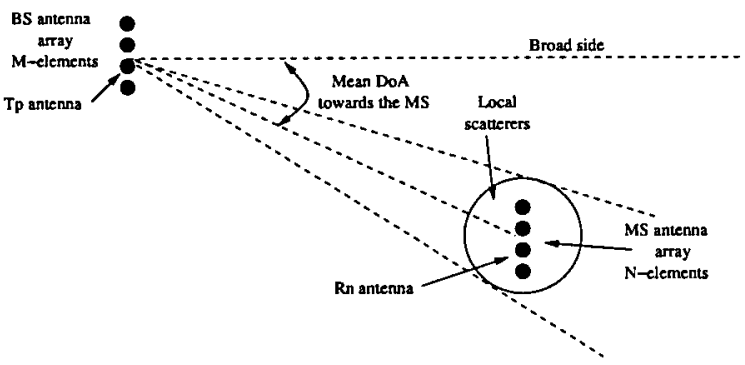

Figure 3.3: Sketch of a scenario where all scatterers are located near the MS so the impinging field at the $B S$ is confined to a narrow azimuth region with a well defined mean direction-of-arrival.

the $p$ th antenna element at the BS, $R n$ is the $n$th antenna element at the MS, $D$ is the distance between the BS and the MS. The effective scatterer on the ring at the MS is denoted by $S(a)$ and $a$ is the angle between the scatterer and the array at the MS. In the model we assume that $S(a)$ are uniformly distributed in $a$ and the phase shift $\phi(a)$ associated with each scatterer, are distributed uniformly over $[-\pi, \pi]$ and IID in $a$. The complex channel coefficient between the $p$ th element at the BS and the $n$th element at the MS, if there are $K$ effective scatterers $S(a)$ distributed on the ring of the MS, can be expressed as

$$
h_{p, n}=\frac{1}{\sqrt{K}} \sum_{k=1}^{K} \exp \left\{-j 2 \pi / \lambda\left(D_{T p \rightarrow S\left(a_{k}\right)}+D_{S\left(a_{k}\right) \rightarrow R n}\right)+j \phi\left(a_{k}\right)\right\}
$$

The two-ring model [121] considers a MIMO scenario where both the BS and MS are sur- 
rounded by objects resulting in local scattering at the both ends of the radio link (which is generally the case in indoor environments). A depiction of the two-ring model is presented in Figure 3.4. The effective scatterer on the ring at the BS is denoted by $S 1(a)$ and $a$ is the angle between the scatterer and the array at the BS. The effective scatterer on the ring at the MS is denoted by $S 2(b)$ and $b$ is the angle between the scatterer and the array at the MS. In the model we assume that $S 1(a)$ and $S 2(b)$ are uniformly distributed in $a$ and $b$ respectively and the phase shifts, $\phi_{1}(a)$ and $\phi_{2}(b)$ associated with each scatterer, are distributed uniformly over $[-\pi, \pi]$ and IID in $a$ and $b$ respectively. The complex channel coefficient between the $p$ th element at the BS and the $n$th element at the MS, if there are $K 1$ and $K 2$ effective scatterers $S 1(a)$ and $S 2$ (b) distributed on the rings of the BS and MS respectively, can be expressed as

$$
\begin{aligned}
h_{p, n}=\frac{1}{\sqrt{K_{1} K_{2}}} \sum_{k=1}^{K 1} \sum_{l=1}^{K 2} \exp \left\{-j 2 \pi / \lambda\left(D_{T p \rightarrow S 1\left(a_{k}\right)}\right.\right. & \\
\left.+D_{S 1\left(a_{k}\right) \rightarrow S 2\left(b_{l}\right)}+D_{S 2\left(b_{l}\right) \rightarrow R n}\right) & \left.+j \phi_{1}\left(a_{k}\right)+j \phi_{2}\left(b_{l}\right)\right\}
\end{aligned}
$$

where $D_{X \rightarrow Y}$ denotes the distance between $X$ and $Y$ and $\lambda$ is the wavelength.

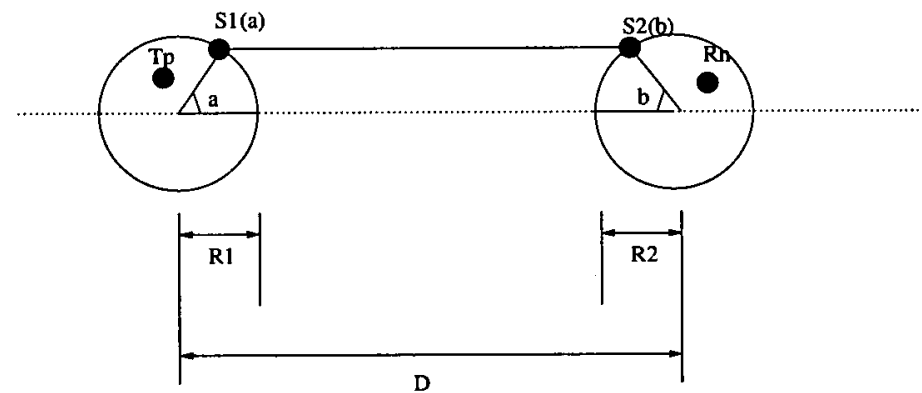

Figure 3.4: Sketch of a two ring scenario where both the $B S$ and $M S$ are surrounded by scatterers.

\subsubsection{Von Mises Angular Distribution}

In [116], a general space time cross-correlation function for mobile frequency nonselective Rice (Ricean) fading MIMO channels is proposed. This model takes into account various parameters of interest such as the angle spreads at the base station and the user, the distance between the base station and the user, mean directions of the signal arrivals, array configurations, and 
Doppler spread. The new space time cross-correlation function includes all the relevant parameters of the MIMO fading channel in a clean compact form, suitable for both mathematical analysis and numerical calculations or simulations. It also covers many other correlation models as special cases.

\subsubsection{Distributed Scattering Model}

In [21] [23], an narrowband model for an outdoor NLOS MIMO radio channel was proposed. Figure 3.5 depicts a NLOS outdoor scenario. Fading is included by the presence of scatterers at both ends of the communication link. The model considers the effect of near-field scatterers only, i.e., scatterers which are either in the vicinity (typically a few tens to hundreds of meters away) of the transmitter or the receiver. Remote scatterers are ignored, assuming that the path loss will tend to limit their contribution to the overall channel. In addition, frequency-flat fading channel is considered. It is assumed that there are $S$ scatterers on both sides of the link, where $S$ is an arbitrary, large enough number for random fading to occur. We consider $N_{T}$ transmit and $N_{R}$ receive antenna elements. The scatterers at the receiver are viewed as an array of virtual antennas located between the transmitter and receiver. The MIMO channel model is given by

$$
\boldsymbol{H}=\frac{1}{\sqrt{S}} \boldsymbol{R}_{\theta_{r}, d_{r}}^{1 / 2} \boldsymbol{G}_{r} \boldsymbol{R}_{\theta_{s}, 2 D_{r} / S}^{1 / 2} \boldsymbol{G}_{t} \boldsymbol{R}_{\theta_{t}, d_{t}}^{1 / 2}
$$

where $G_{t}\left(S \times N_{T}\right)$ and $G_{r}\left(N_{R} \times S\right)$ are random matrices with IID zero mean complex Gaussian elements. $\boldsymbol{R}_{\theta_{r}, d_{r}}, \boldsymbol{R}_{\theta_{s}, 2 D_{r} / S}, \boldsymbol{R}_{\theta_{t}, d_{t}}$ are the correlation matrices seen from the transmitter, virtual array, and receiver respectively. An interesting feature of this channel model (equation 3.11) is that it does not necessarily give Gaussian distributions of the channel coefficients [23].

\subsubsection{Remarks on MIMO Channel Models}

The non-physical models are essentially statistical models, using non-physical parameters. These type of models offer advantages, such as implementation simplicity and accuracy in characterization of the MIMO propagation channels for the scenarios they describe. Moreover, they can be easily simulated and therefore they can assist in analyzing and designing MIMO systems. However, they only provide a narrow insight to the scattering characteristics of the MIMO radio channel and are dependant upon the measurement results. Note, that the accuracy 


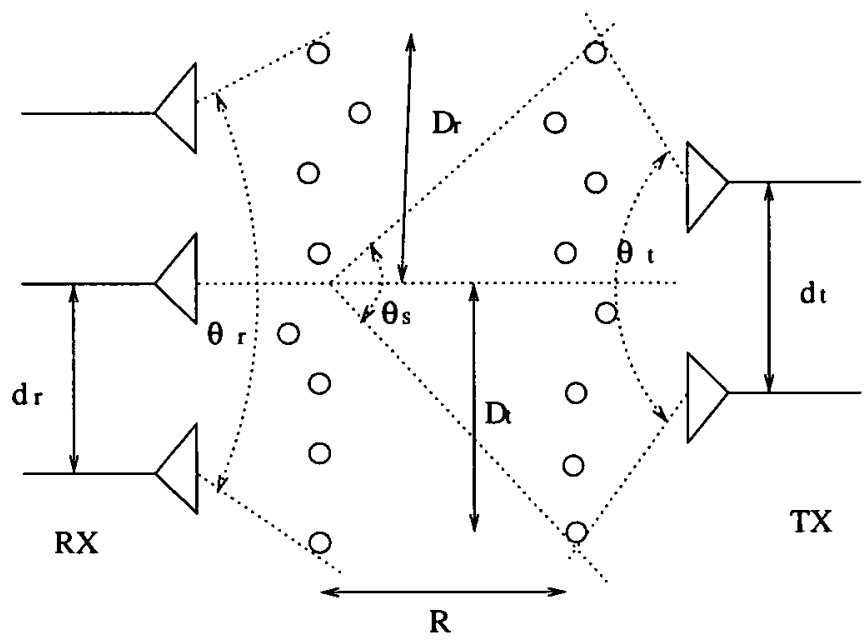

Figure 3.5: Illustration of the distributed scattering model.

of the measurements is affected greatly by the measurement setups and equipments.

On the other side, the physical models create a realistic geometric multipath propagation model in order to portray as precisely as possible the MIMO scattering environment. For this purpose, some important physical parameters are selected and are used to determine the distribution of the scatterers. The most popular parameters are AOA (Angle Of Arrival), AOD (Angle Of Departure) and TOA (Time Of Arrival). In the following sections, we will focus on the nonphysical MIMO channel model we have used in this thesis.

\subsection{Stochastic MIMO Channel Model}

A detailed description of the stochastic radio channel adopted in this thesis can be found in [104] and [107]. Assume that the transmitter is the BS and the receiver is the MS. All antenna elements in the two arrays have the same polarization and the same radiation pattern. The spatial complex correlation coefficient at the BS between antenna $m_{1}$ and $m_{2}$, assuming that the BS antenna array is elevated above the local scatterers, is given by

$$
\rho_{m_{1} m_{2}}^{B S}=<h_{m_{1} n}, h_{m_{2} n}>
$$

where $\langle a, b\rangle$ computes the correlation coefficient between $a$ and $b$. From (3.12) it is assumed that the spatial correlation coefficient at the BS is independent of $n$, since the $n$ elements at the 
MS, illuminate the same surrounding scatterers and, therefore, also generate the same power azimuth spectrum (PAS) as the BS. The spatial correlation function is the Fourier transform of the PAS [122]. Different expressions of the spatial correlation function have been derived in the literature assuming that the PAS follows some specific functions [123] [124]. The spatial complex correlation coefficient observed at the MS is similarly defined as

$$
\rho_{n_{1} n_{2}}^{M S}=<h_{m n_{1}}, h_{m n_{2}}>
$$

and assumed to be independent of $m$.

Given (3.12) and (3.13), the following symmetrical complex correlation matrices can be defined

$$
\boldsymbol{R}_{B S}=\left[\begin{array}{cccc}
\rho_{11}^{B S} & \rho_{12}^{B S} & \cdots & \rho_{1 N_{T}}^{B S} \\
\rho_{21}^{B S} & \rho_{22}^{B S} & \cdots & \rho_{2 N_{T}}^{B S} \\
\vdots & \vdots & \ddots & \vdots \\
\rho_{N_{T} 1}^{B S} & \rho_{N_{T} 2}^{B S} & \cdots & \rho_{N_{T} N_{T}}^{B S}
\end{array}\right]_{N_{T} x N_{T}}
$$

and

$$
\boldsymbol{R}_{M S}=\left[\begin{array}{cccc}
\rho_{11}^{M S} & \rho_{12}^{M S} & \cdots & \rho_{1 N_{R}}^{M S} \\
\rho_{21}^{M S} & \rho_{22}^{M S} & \cdots & \rho_{2 N_{R}}^{M S} \\
\vdots & \vdots & \ddots & \vdots \\
\rho_{N_{R} 1}^{M S} & \rho_{N_{R}}^{M S} & \cdots & \rho_{N_{R} N_{R}}^{M S}
\end{array}\right]_{N_{R} x N_{R}}
$$

The correlation coefficient between two arbitrary transmission coefficients connecting two different sets of antennas is expressed as

$$
\rho_{n_{2} m_{2}}^{n_{1} m_{1}}=<h_{m_{1} n_{1}}, h_{m_{2} n_{2}}>
$$

which is equivalent (proof presented in [107]) to

$$
\rho_{n_{2} m_{2}}^{n_{1} m_{1}}=\rho_{n_{1} n_{2}}^{M S} \rho_{m_{1} m_{2}}^{B S}
$$

this means that the spatial correlation matrix of the MIMO radio channel is the Kronecker product of the spatial correlation matrix at the MS and the BS and is given by 


$$
\boldsymbol{R}_{M I M O}=\boldsymbol{R}_{M S} \otimes \boldsymbol{R}_{B S}
$$

\subsection{Generation of Simulated Correlated Channel Coefficients}

Correlated channel coefficients $h_{m n}$ are generated from zero-mean complex independent identically distributed (i.i.d.) random variables $d_{m n}$ shaped by the desired Doppler spectrum such that [107]

$$
\boldsymbol{H}=\boldsymbol{C d}
$$

where $\boldsymbol{H}_{N_{T} N_{R} x 1}=\left[h_{11}, h_{21}, \ldots, h_{N_{T} 1}, h_{12}, \ldots, h_{N_{T} N_{R}}\right]^{T}$ and $\boldsymbol{d}_{N_{T} N_{R} x 1}=\left[d_{1}, d_{2}, \ldots, d_{N_{T} N_{R}}\right]^{T}$. The symmetrical mapping matrix $\boldsymbol{C}$ results from the standard Cholesky factorization of the matrix $\boldsymbol{R}_{M I M O}=\boldsymbol{C} \boldsymbol{C}^{T}$ provided that $\boldsymbol{R}_{M I M O}$ is nonsingular [125]. The generation of the simulated MIMO channel matrix $\tilde{\boldsymbol{H}}$ can be deduced from the vector $\boldsymbol{H}$.

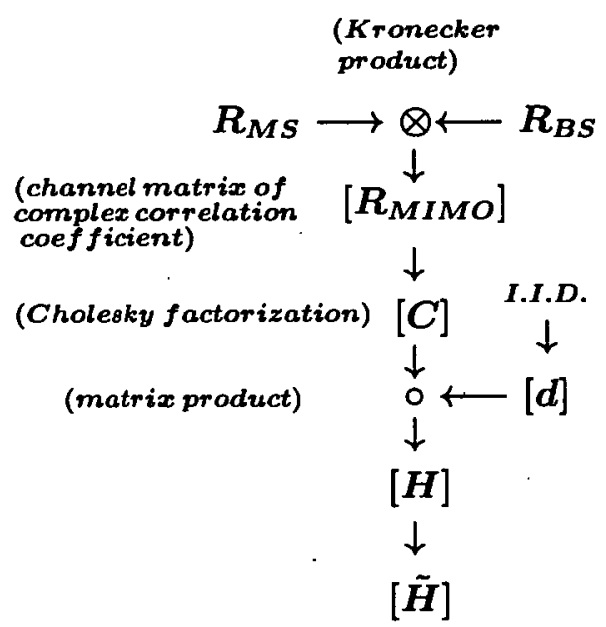

Figure 3.6: Flow chart illustrating the practical procedure to obtain correlated channel coefficient.

The flow chart illustrating the complete procedure to generate correlated channel coefficient is presented in Figure 3.6. 
Applying the Gaxpy algorithm [125] p.143 (Cholesky decomposition) to $\boldsymbol{R}_{M I M O}$ we get the lower-triangular matrix $C$. Let $d$ be a vector of $N_{T} N_{R}$ complex zero-mean unit variance independent random variables. As described in equation (3.18), correlated channel coefficients $h_{m n}$ are generated from the $N_{T} \times N_{R}$-vector $d$ and the matrix $C$.

\subsection{Detection Algorithms}

\subsubsection{Zero-Forcing Equalizer (ZF)}

Zero-forcing passes the received signal $r_{1}$ through the inverse channel in order to restore the transmitted signal [41]. Figure 3.7 illustrates in block diagram the equivalent discrete-time channel and equalizer.

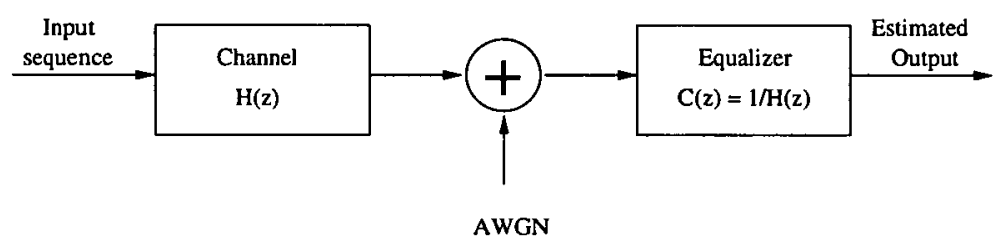

\{v\}

Figure 3.7: Block diagram of channel with zero-forcing equalizer.

The receiver vector $\boldsymbol{r}_{1}$ is multiplied with the inverse of channel matrix $\boldsymbol{H}$. The pseudo-inverse of $\boldsymbol{H}: \boldsymbol{H}^{+}=\left(\boldsymbol{H}^{\boldsymbol{H}} \boldsymbol{H}\right)^{-1} \boldsymbol{H}^{\boldsymbol{H}}$ is used. Where $\boldsymbol{H}^{\boldsymbol{H}}$ represents the the Hermitian (conjugate) transpose of $\boldsymbol{H}$.

$$
H^{+} r_{1}=H^{+} H a+H^{+} v \Rightarrow \hat{a}=a+n^{\prime}
$$

where $\hat{a}$ represents the the symbol vector $N_{T} \times 1$, recovered at reception. $\hat{a}$ consists of the emitted signal $\boldsymbol{a}$ and a colored noise $\boldsymbol{n}^{\prime}$. As $\hat{\boldsymbol{a}}$ is distorted by $\boldsymbol{n}^{\prime}=\boldsymbol{H}^{+} \boldsymbol{v}$ the performance of Zero-Forcing depends largely of the conditioning of $\boldsymbol{H}$. The $k_{i}$ th ZF-nulling vector $\boldsymbol{w}_{k_{i}}$ is defined as the unique minimum norm vector satisfying

$$
\boldsymbol{w}_{k_{i}}{ }^{T}(\boldsymbol{H})_{k_{j}}= \begin{cases}0 & j \neq i \\ 1 & j=i\end{cases}
$$

Where the $(\boldsymbol{H})_{k_{j}}$ denotes the the $k_{j}$ th column of $\boldsymbol{H}$. 


\subsubsection{Minimum Mean Squared Error Detector (MMSE)}

The MMSE detector [108] [109] is a type of linear detector. It can be obtained if a linear transformation is sought which minimizes the mean square error between the transmitted symbols and the outputs of the transformation. The detector is represented by an $N_{T} \times N_{R}$ matrix $C_{M M S E}$ which minimizes :

$$
\sum_{k=1}^{N_{T}} E\left[\left\|\left(C_{M M S E} r_{1}-a\right)_{k}\right\|^{2}\right]
$$

If the background noise covariance $N_{0}>0$ then the MMSE detector may be represented by

$$
C_{M M S E}=\sqrt{P} \boldsymbol{H}^{H}\left[P \boldsymbol{H} \boldsymbol{H}^{H}+N_{0} I_{N_{R}}\right]^{-1}
$$

where $(\bullet)^{H}$ denotes the conjugate transpose, and $I_{N_{R}}$ is the $N_{R} \times N_{T}$ identity matrix. $P$ is the total transmitted power.

The MMSE estimate can be calculated by the following equation

$$
\hat{\boldsymbol{a}}=C_{M M S E} \cdot \boldsymbol{r}_{1}
$$

\subsubsection{VBLAST Detection Algorithm}

Flat fading MIMO channels having multiple transmit and receive antennas were shown to offer large spectral efficiencies compared to SISO (single input single output) channels [17], [16]. Capacity increases linearly with the number of transmit antennas as long as the number of receive antennas is greater than or equal to the number of transmit antennas. To achieve this capacity, Diagonal BLAST (Bell Labs 1Ayered Space-Time) architecture was proposed by Foschini [17]. This scheme utilizes multi-element antenna arrays at both ends of wireless link. The D-BLAST encoder uses a space time arrangement that corresponds to a diagonal layering. The information bit stream coming from the source is demultiplexed into several substreams (serial to parallel), and each substream is coded separately and mapped to complex symbols. Then the symbols of each substream are dispersed diagonally across antennas and time. Figure 3.8(a) shows the antenna and instant where symbols associated to each layer are transmitted, for a 
system with four transmit antennas (figure adapted from [17], fig. 6). Note that the layer might have more symbols than the number of transmit antennas, and the frame can be very long. Unfortunately, given the structure of the decoder, the space time wastage is necessary. This ultimately makes D-BLAST unable to reach the capacity limit, since the wastage is repeated every time a new set of layers are to be transmitted. Note that since the symbols are spread across antennas, this scheme captures transmit diversity.

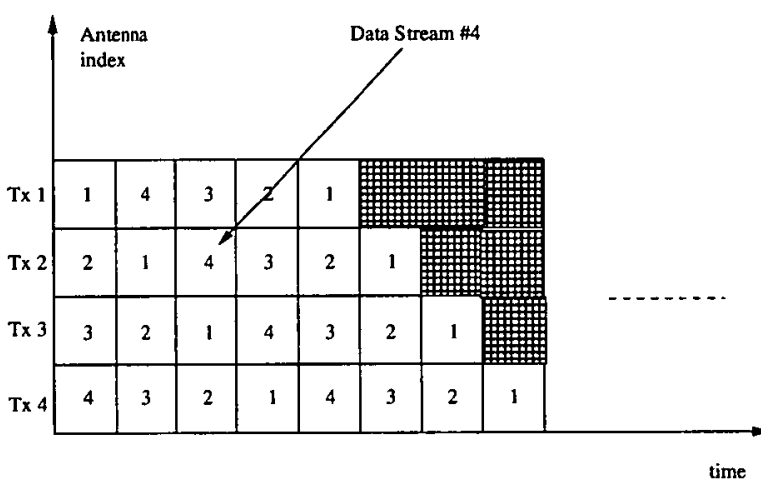

(a)

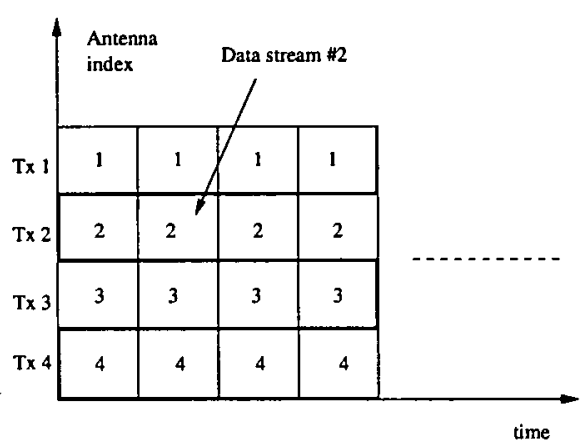

(b)

Figure 3.8: Numbers in blocks represent the layer that can transmit its symbols at that antenna and symbol period. Filled blocks represent space time wastage: (a) D-BLAST: diagonal layering, (b) VBLAST: horizontal layering

The complexities of D-BLAST implementation led to V-BLAST (Figure3.8(b)) which is a modified version of BLAST [110]. As in D-BLAST, the information bit stream is separated in substreams, and each can undergo its own channel coder. However, the layering is horizontal, meaning that all the symbols of a certain stream are transmitted through the same antenna (one stream per antenna). This eliminates the space time wastage, but loses the transmit diversity, since each stream is tied to its antenna. Two nulling criteria, namely zero-forcing [55] and minimum mean square error (MMSE) [126], are utilized as detection algorithms. Originally, the BLAST detection scheme was based on a successive interference cancellation (SIC) [55] [110] [127]. The incorporation of SIC and MMSE achieves the Shannon MIMO capacity. A parallel interference cancellation scheme was also proposed later [128].

BLAST detectors including both parallel interference cancellation (PIC) and SIC suffer from the error propagation problem. They can give poor energy efficiency if the previously detected layers are not perfectly cancelled, because the following layers experience more interference if the previously detected signals are not cancelled correctly. The error propagation problem 
of BLAST detectors can be reduced with channel coding and interleaving [129][130]. In this section VBLAST detection algorithm is described ([131]).

Let the ordered set

$$
S=\left\{k_{1}, k_{2}, \ldots, k_{N_{T}}\right\}
$$

be a permutation of the integers $1,2, \ldots, N_{T}$ specifying the order in which components of the transmitted symbol vector $a$ are extracted. The detection algorithm operates on $r_{1}$, progressively computing decision statistics $y_{k_{1}}, y_{k_{2}}, \ldots, y_{k_{N_{T}}}$, which are then sliced to form estimates of the underlying data symbols $\hat{a}_{k_{1}}, \hat{a}_{k_{2}}, \ldots, \hat{a}_{k_{N_{T}}}$. Thus, decision statistic $y_{k_{1}}$ is computed first then $y_{k_{2}}$, and so on. To determine a particular ordering $S_{o p t}$ which is optimal in a certain sense we calculate a metric for each transmit antenna. For now we assume an arbitrary ordering $S$. The detection process uses linear combining nulling and symbol cancellation to successively compute the $y_{k_{i}}$, proceeding generally as follows:

Step 1: Using nulling vector $\boldsymbol{w}_{\boldsymbol{k}_{1}}$, form a linear combination of the components of $\boldsymbol{r}_{1}$ to yield $y_{k_{1}}$ :

$$
y_{k_{1}}=\boldsymbol{w}_{k_{1}}^{T} r_{1}
$$

Step 2: Slice $y_{k_{1}}$ to obtain $\hat{a}_{k_{1}}$

$$
\hat{a}_{k_{1}}=Q\left(y_{k_{1}}\right)=\frac{y_{k_{1}}}{\boldsymbol{w}_{k_{1}}^{T}(\boldsymbol{H})_{k_{1}}}
$$

where $Q($.$) denotes the quantization (slicing) operation appropriate to the constellation in use$ and $(\boldsymbol{H})_{k_{1}}$ denotes the $k_{1}$ th column of $\boldsymbol{H}$.

Step 3: Assuming that $\hat{a}_{k_{1}}=a_{k_{1}}$, cancel $a_{k_{1}}$ from the received vector $\boldsymbol{r}_{1}$, resulting in a modified received vector $\boldsymbol{r}_{\mathbf{2}}$ :

$$
r_{2}=r_{1}-\hat{a}_{k_{1}}(H)_{k_{1}}
$$


Steps 1-3 are then performed for components $k_{2}, \ldots, k_{N_{T}}$ by operating in turn on the progression of modified received vectors $r_{2}, r_{3}, \ldots, r_{N_{T}}$.

The most common choices for the criterion chosen to compute the nulling vectors $\boldsymbol{w}_{\boldsymbol{k}_{i}}$ are the mean-squared error (MMSE) and zero-forcing (ZF) criteria. Both of these criteria are described and implemented here.

The ZF criterion: The zero forcing (see chapter 3.6.1) block linear equalization is based on nulling all other users by using the pseudo-inverse in order to make a decision about one user. The algorithm is thoroughly discussed in [132]. The $k_{i}$ th ZF-nulling vector is orthogonal to the subspace spanned by the contributions to $r_{k_{i}}$ due to those symbols not yet estimated and cancelled. It is not difficult to show that the unique vector satisfying equation (3.20) : $\boldsymbol{w}_{k_{i}}{ }^{T}(\boldsymbol{H})_{k_{j}}=\left\{\begin{array}{l}0 j \neq i \\ 1 j=i\end{array}\right.$

is just the $k_{i}$ th row of $\boldsymbol{H}_{\bar{k}_{i-1}}^{+}$where the notation $\boldsymbol{H}_{\overline{k_{i}}}$ denotes the matrix obtained by zeroing columns $k_{1}, k_{2}, \ldots, k_{i}$ of $\boldsymbol{H}$ and ${ }^{+}$denotes the Moore-pseudoinverse [125]. One problem with $\mathrm{ZF}$ is that it might cause noise enhancement and degrade performance, if $\tilde{\boldsymbol{H}}$ is poorly conditioned.

The MMSE criterion: The minimum mean square error.(MMSE) technique employs a more practical criterion for linear detection to achieve an improved performance compared to ZF. MMSE includes the noise power in filter calculations and instead of nulling all users, it attenuates them to the noise level and in this way controls the noise enhancement problem encountered in ZF. VBLAST MMSE differs from the VBLAST-ZF in that the nulling vector derives from the solution of the equation (3.21), which is actually defined in equation (3.22): $C_{M M S E}=\sqrt{P} \boldsymbol{H}^{\boldsymbol{H}}\left[P \boldsymbol{H} \boldsymbol{H}^{\boldsymbol{H}}+\frac{N_{0}}{\rho}\right]^{-1}$

\subsection{Simulation}

Bit error ratio (BER) versus signal-to-noise ratio (SNR) simulations were carried out in order to compare the performance of the MMSE and VBLAST MMSE for different degrees of correlation in the radio channel. Note that the SNR is the SNR per symbol averaged over each receive antenna. Two types of modulation have been used : BPSK and 16-QAM. The effect of error propagation (EP) in the V-BLAST is also investigated. The curves labeled NEP (no error prop- 
agation) were obtained by implementing perfect cancellation by subtraction. Signal-to-noise ratios between $-10 \mathrm{~dB}$ and $20 \mathrm{~dB}$ were considered. The SNR of $v \mathrm{~dB}$ corresponds to

$$
\frac{P}{N_{0}}=10^{\frac{v}{10}}
$$

where $N_{0}$ is the variance of the noise vector as defined in Section 3.2 and $P$ is the average transmitted power.

For all the simulations, each frame was of length 100. Each frame corresponds to one simulated channel and $100 N_{T}$ transmitted symbols. Each point plotted is the average of 10000 simulated channels.

\subsubsection{Statistical Confidence}

An interesting point that arises when measuring the BER is the statistical confidence in the results. The statistical confidence coefficient [133] is defined as the probability, based on a set of measurements, that the actual probability of an event is in a specified interval $\left(c_{1}, c_{2}\right)$. When applied to BER measurements, the definitions of the statistical confidence coefficient can be restated as the probability (based on $e$ detected errors out of $n$ bits transmitted) that the actual number of bit error $e$ is in a specified interval $\left(k_{1}, k_{2}\right)$. Mathematically, this can be expressed as [133]

$$
C C=P\left[k_{1} \leq e \leq k_{2}\right]
$$

where $P[]$ indicates probability and $\mathrm{CC}$ is the confidence coefficient. Because confidence coefficient is a probability by definition, the possible values range from $0 \%$ to $100 \%$. After computing the confidence coefficient, we can say we have $\mathrm{CC}$ percent confidence that the $e$ is in the interval $\left(k_{1}, k_{2}\right)$.

Calculations of the confidence level are based on the binomial distribution function described in many statistics texts [133]. The binomial distribution function is generally written as

$$
P_{n}(k)=\frac{n !}{k !(n-k) !} p^{k} q^{n-k}
$$


Equation 3.30 gives the probability that $k$ events (i.e., bit errors) occur in $n$ trials (i.e., $n$ bits transmitted), where $p$ is the probability of event occurrence in a single trial (i.e., a bit error), and $q$ is the probability that the event does not occur in a single trial (i.e., no bit error). Note that the binomial distribution models events that have two possible outcomes, such as error/no error. Thus,

$$
p+q \doteq 1
$$

Also, according to [133], the mean value $\mu$ and the variance $\sigma^{2}$ of the binomial distribution for $n \rightarrow \infty$ is given by the follow expressions

$$
\begin{gathered}
\mu=n p \\
\sigma^{2}=n p q
\end{gathered}
$$

When we are interested in the probability that $N$ or fewer events occur in $n$ trials, then the cumulative binomial distribution function can be expressed as [134]

$$
P(e \leq N)=\sum_{k=0}^{N} P_{n}(k)=\sum_{k=0}^{N} \frac{n !}{k !(n-k) !} p^{k} q^{n-k}
$$

In terms of the cumulative binomial distribution function, the confidence coefficient is defined as

$$
C C=P\left(k_{1} \leq e \leq k_{2}\right)=\sum_{k=k_{1}}^{k_{2}} \frac{n !}{k !(n-k) !} p^{k}(1-p)^{n-k}
$$

A MATLAB implementation of this model has been used to calculate the prediction point of BER. Specifically, equation (3.35) has been computed for a hypothetical value of $p$. We choose $p=10^{-4}$. The other parameters needed are $n=10^{6}$ (total bits transmitted), $k_{1}=\mu-\sigma=90$ (down confident limit) and $k_{2}=\mu+\sigma=110$ (up confident limit). In that case we achieve a confidence coefficient of $95 \%$. This means that $95 \%$ of the times the measured bit errors for 
$n=10^{6}$ transmitted bits will be between 90 and 110 errors. Note that the actual bit errors (for the assumed probability of bit error $p=10^{-4}$ ) is expected to be 100 errors per $n=10^{6}$ transmitted bits. We need to count 100 errors to reach a reasonable reliability. Note that in our simulations we did not consider bit error rate lower than $10^{-3}$.

\subsubsection{Simulation Setup}

To introduce correlation into the simulation scenarios we have used the MIMO theoretical radio channel that results after the application of the algorithm described previously (Section 3.5). In order to validate the stochastic MIMO model we have used channel correlation figures resulting from measurements [107]. The input parameters used in the validation stage are the average spatial complex correlation matrices $\boldsymbol{R}_{B S}$ and $\boldsymbol{R}_{M S}$. The measured spatial complex correlation matrices are the results of an average over the reference transmitting and receiving antennas. Two real examples have been used as input.

Example 1: Picocell Decorrelated. See (3.36.)

\section{Example 2: Microcell Correlated. See (3.37.)}

In examples 1 and $2, \boldsymbol{R}_{M S}$ is decorrelated. This is expected since the MS is surrounded by scatterers. On the other hand, $\boldsymbol{R}_{B S}$ represents two different behaviours. In Example 1, the spatial correlation coefficient remains low as expected in the case of an indoor termination. On the other hand, the spatial correlation coefficients at the BS are larger in Example 2 with a mean absolute value of the coefficient equal to 0.96 . The high correlation is explained by the fact that in this specific example the BS is located above any surrounding scatterer. Therefore, a low azimuth spread (AS) is expected, which causes the antenna array elements to be highly correlated.

$$
\begin{aligned}
& \boldsymbol{R}_{B S}=\left[\begin{array}{cccc}
1 & -0.45+0.53 j & 0.37-0.22 j & 0.19+0.21 j \\
-0.45-0.53 j & 1 & -0.35-0.02 j & 0.02-0.27 j \\
0.37+0.22 j & -0.35+0.02 j & 1 & -0.10+0.54 j \\
0.19-0.21 j & 0.02+0.27 j & -0.10-0.54 j & 1
\end{array}\right] \\
& \boldsymbol{R}_{M S}=\left[\begin{array}{cccc}
1 \\
1 \\
-0.13+0.62 j & -0.13-0.62 j & -0.49+0.23 j & 0.15+0.28 j \\
-0.49-0.23 j & -0.13+0.52 j & -0.13-0.52 j & -0.38+0.12 j \\
0.15-0.28 j & -0.38-0.12 j & 0.02+0.61 j & 0.02-0.61 j \\
- & 1 & 1
\end{array}\right]
\end{aligned}
$$




$$
\begin{aligned}
& \boldsymbol{R}_{B S}=\left[\begin{array}{cccc}
1 & -0.61+0.77 j & 0.14-0.94 j & 0.24+0.89 j \\
-0.61-0.77 j & 1 & -0.85+0.50 j & 0.57-0.78 j \\
0.14+0.94 j & -0.85-0.50 j & 1 & -0.91+0.40 j \\
0.24-0.89 j & 0.57+0.78 j & -0.91-0.40 j & 1
\end{array}\right] \\
& \boldsymbol{R}_{M S}=\left[\begin{array}{cccc}
0.08+0.05 j & -0.02-0.13 j \\
-0.12+0.18 j & -0.12-0.18 j & 0.08+0.11+0.04 j \\
0.08-0.05 j & -0.17+0.16 j & -0.17-0.16 j & 0.11+0.17-0.16 j \\
-0.02+0.13 j & 0.11-0.04 j & -0.17+0.16 j & 1
\end{array}\right]
\end{aligned}
$$

\subsubsection{Results}

In this Section, the performances (in terms of BER) of different MIMO systems are evaluated and compared with each other. We separate the results into five major categories according to the following parameters.

1. Modulation schemes (BPSK vs. 16-QAM)

2. Detection techniques (VBLAST MMSE vs. MMSE)

3. number of transmit and receive antennas

4. Error Propagation (EP vs. NEP)

5. spatial fading complex correlation

all graphs present the BERs versus SNR for different scenarios.

\subsubsection{Modulation Schemes}

In this section we investigate the effect of two different modulation schemes on a MIMO $(4,4)$ system. Figures 3.9(a) and (b) show the constellation diagrams for the Gray-coded 16-QAM and Gray-coded BPSK respectively. Quadrature amplitude modulation (QAM) is a modulation scheme which conveys data by changing (modulating) the amplitude of two carriers. These two carrier waves, usually sinusoids, are out of phase with each other by $90^{\circ}$ and are thus called 
quadrature carriers [41]. Binary phase-shift keying (BPSK) is the simplest form of PSK. It uses two phases which are separated by $180^{\circ}$ and so can also be termed 2-PSK. It does not particularly matter exactly where the constellation points are positioned, and in Figure 3.9 (b) they are shown on the real axis, at $0^{\circ}$ and $180^{\circ}$. Phase-shift keying (PSK) is a digital modulation scheme that conveys data by changing, or modulating, the phase of a reference signal (the carrier wave) [41].

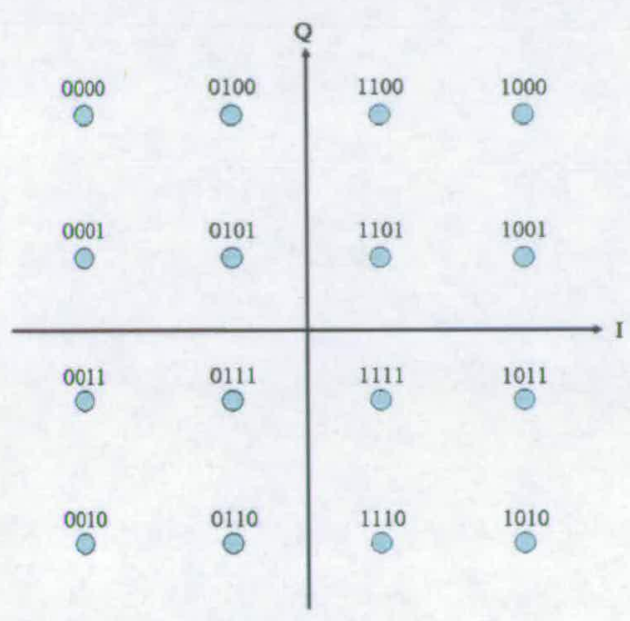

(a)

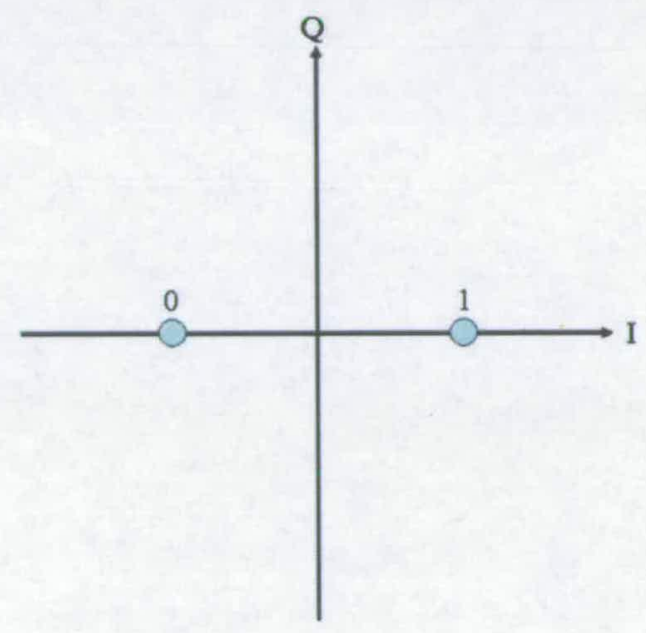

(b)

Figure 3.9: Constellation diagram for: (a) Gray-coded 16-QAM, (b) Gray-coded BPSK

Figure 3.10 shows the numerical evaluation of the average bit error rate (BER) for the case of BPSK and 16-QAM modulation schemes. It is obvious from the graphs that BPSK gives a much better performance, in terms of BER, for the same values of SINR. The explanation for these results is the difference in the order of the constellation between BPSK and 16-QAM. Specifically, BPSK is in fact 2-QAM, which means that BPSK is a lower-order constellation compared to 16-QAM. By moving to a higher-order constellation, it is possible to transmit more bits per symbol. However, if the mean energy of the constellation is to remain the same (by way of making a fair comparison), the points must be closer together and are thus more susceptible to noise and other corruption. This results in a higher bit error rate and so higher-order QAM can deliver more data less reliably than lower-order QAM. The BER is increasing as the order of the constellation increases [41] (see p.281). 


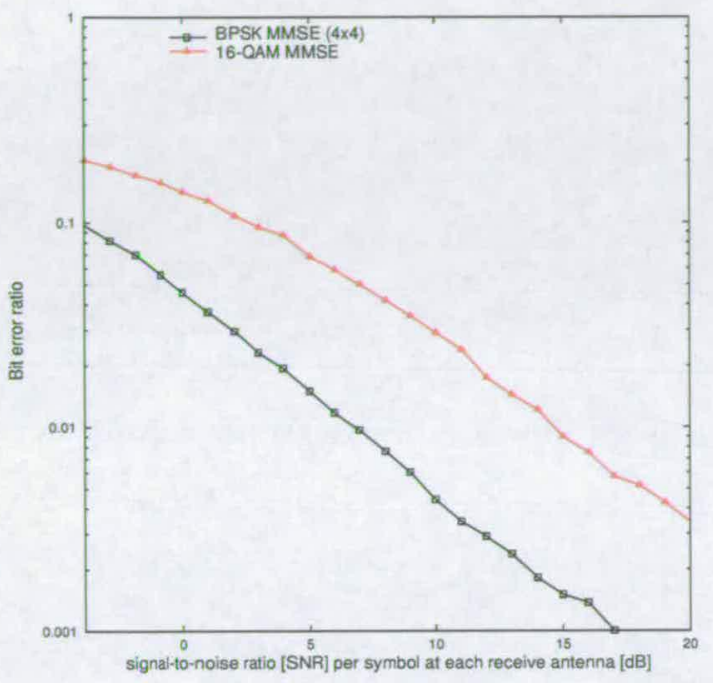

(a)

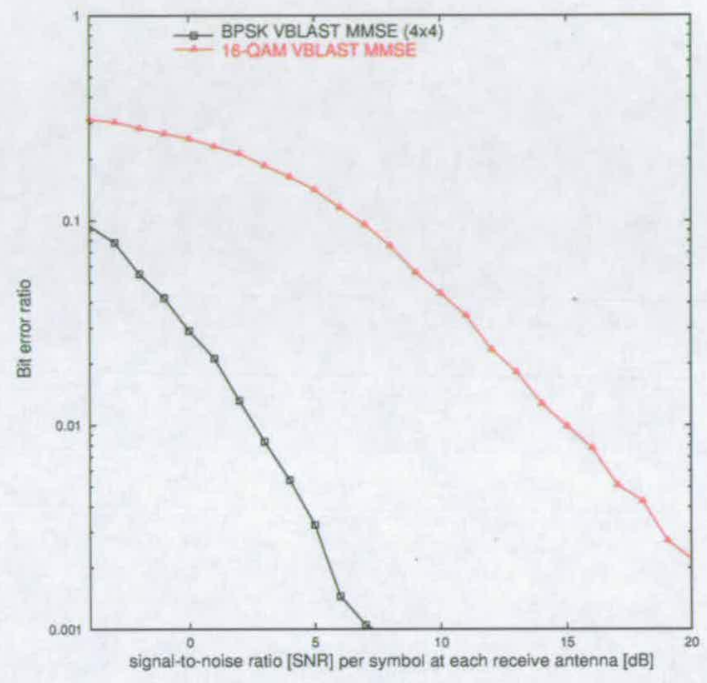

(b)

Figure 3.10: Comparison of MIMO systems for different modulation schemes (BPSK vs. 16-QAM): (a) MMSE detector, (b) VBLAST MMSE detector

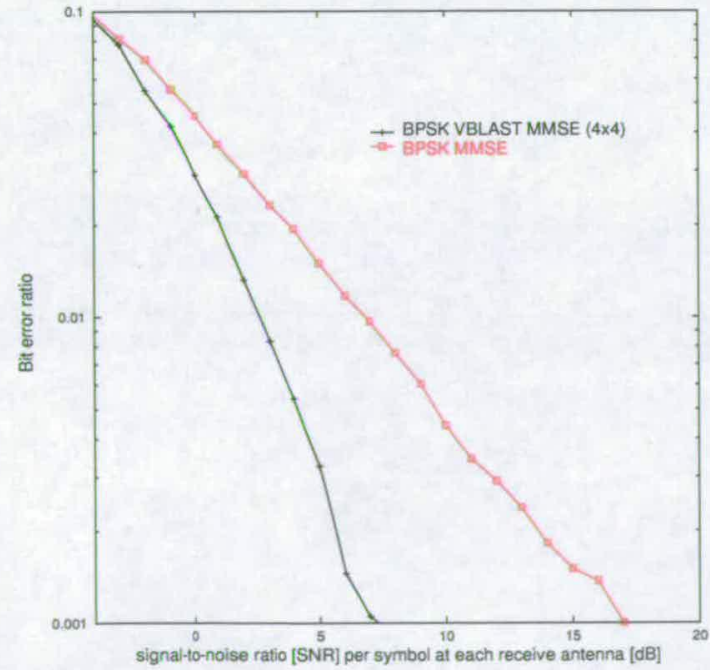

(a)

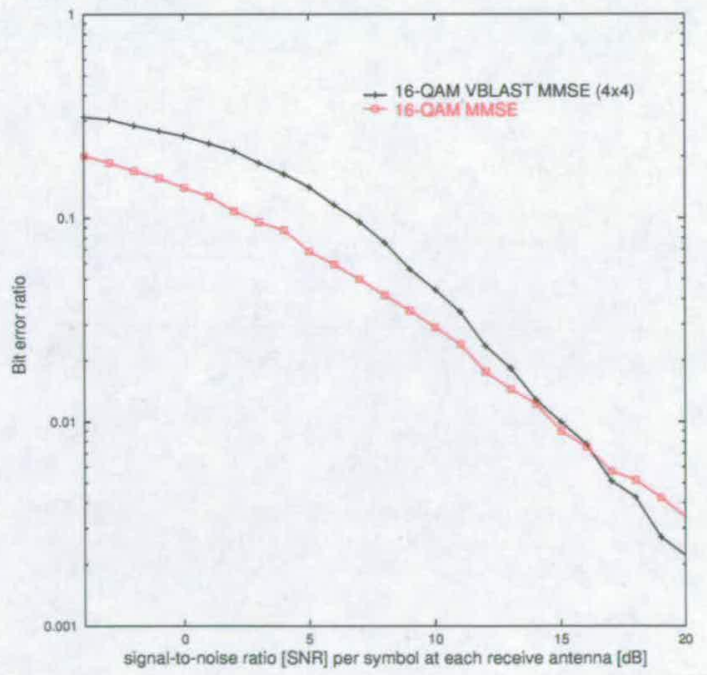

(b)

Figure 3.11: Detection techniques comparison on MIMO $(4,4)$ system: (a) BPSK modulation MMSE/VBLAST MMSE detector, (b) 16-QAM modulation - MMSE/VBLAST MMSE detector 


\subsubsection{Detection Techniques}

In this section we present the effect of two different detection techniques on a MIMO $(4,4)$ system. Figure 3.11 shows the performance, in terms of BER, of MMSE and VBLAST MMSE detectors of a MIMO $(4,4)$ system. The BER is evaluated for two different modulation schemes, BPSK and 16-QAM. It is clear from Figure 3.11(a) that in the case of BPSK modulation the VBLAST MMSE outperforms MMSE. At the BER value equal to 0.001 there is a loss of $10 \mathrm{~dB}$ due to MMSE. In the case of 16-QAM (Figure 3.11(b)), the MMSE detector performs better than the VBLAST MMSE for values of SNR smaller than 15dB. The explanation for this phenomenon is given in the error propagation Section (3.7.3.4).

\subsubsection{Number of Transmit and Receive Antennas}

In this section we present the performance, in terms of BER values, of MIMO systems with different numbers of transmit and receive antennas. In Figure 3.12 we compare the BER values of following MIMO systems: $(1,2),(2,2),(2,4),(4,4),(4,6),(6,6)$. In the cases where the numbers of transmit and receive antennas are the same, we notice that, a MIMO system with more antennas is more efficient in terms of BER values than a MIMO with less antennas. For a target uncoded BER of 0.1 , the SNR values of the MIMO systems $(2,2),(4,4)$, and $(6,6)$ are respectively: $11.5 \mathrm{~dB}, 7 \mathrm{~dB}$ and $5 \mathrm{~dB}$. Namely, going from a MIMO system $(6,6)$ to a $(4,4)$ there is a $2 \mathrm{~dB}$ SNR loss, and going from a MIMO $(4,4)$ to a $(2,2)$ there is a $4.5 \mathrm{~dB}$ SNR loss.

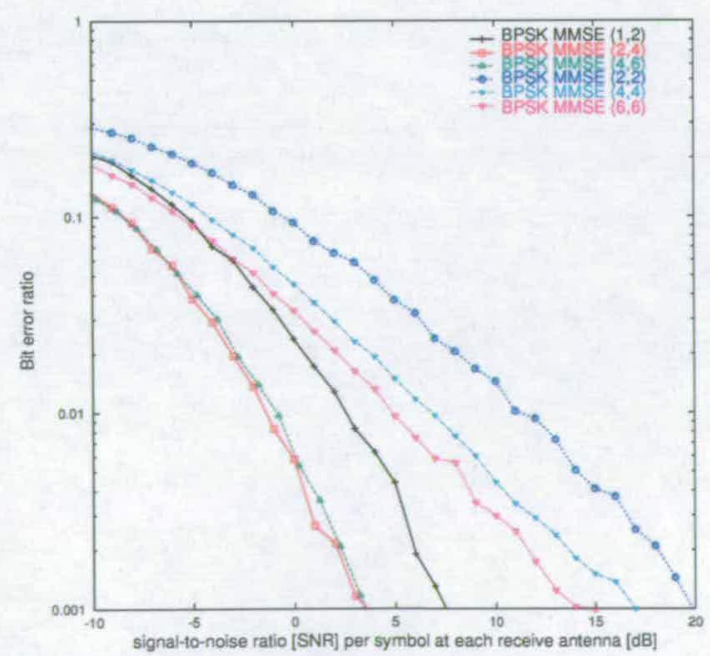

Figure 3.12: Comparison of MIMO systems with different number of antenna arrays at transmitter and receiver, BPSK modulation, MMSE detector 
In the cases where the numbers of transmit and receive antennas are not the same, a MIMO system gives similar BER values, apart from the $(1,2)$ case, where there is only one transmitter. In the case of one transmitter the MMSE detection technique is not exploited properly, because of the absence of different transmitted signals (i.e. no diversity). An MMSE detector is able to incorporate (exact or approximate) knowledge of the received signals, in order detect the received signal, such a feature is not exploited in the case of only one transmitter. Finally, we notice that any MIMO system with more antennas at the receiver than the transmitter $((1,2)$, $(2,4)$ and $(4,6))$ performs better than configurations with the same size of antenna arrays at both the receiver and the transmitter $((2,2),(4,4)$, and $(6,6))$.

\subsubsection{Error Propagation Effect}

In this section we investigate the effect of error propagation (EP) on different MIMO systems. The performance of VBLAST is constrained by the accuracy of the symbols recovered in the previous layer. These decision errors that are fed back result in error propagation. The error propagation problem contributes to the degraded bandwidth efficiency. For simulation of the case without error propagation, the receiver is assumed to have exact knowledge of all symbol layers that are cancelled at each iteration. Specifically, in Figure 3.13 we can see how important is for a MIMO $(4,4)$ system the presence of error propagation. The effect is more notable when we apply the 16-QAM modulation scheme and this will be discussed further below.

Figure 3.14 shows how crucial is the impact of error propagation, in terms of BER, in a MIMO $(4,4)$ system using 16-QAM modulation and VBLAST MMSE detection. Error propagation, specially in the lower SNRs, leads VBLAST detection method to perform worse than the MMSE detection. The presence of EP propagation results in a 7dB SNR loss for VBLAST MMSE EP against VBLAST MMSE NEP at the BER value equal to 0.1. Also, There is also a $9 \mathrm{~dB}$ loss at the BER value equal to 0.01 . An explanation why the error propagation affects more 16-QAM modulation than BPSK, can be seen in the constellation structure. As we can see from Figures 3.9(a) and (b) for the same values of SNR the distance among the neighbouring constellation points is smaller in the case of 16-QAM compared to the BPSK constellation. This leads 16-QAM constellation to be more sensitive to interference than in the BPSK case. Specifically in the VBLAST MMSE detection, the signal must be detected successfully for it to be subtractively cancelled correctly. Otherwise error propagation can occur, so that the potential performance enhancement is not achieved. 


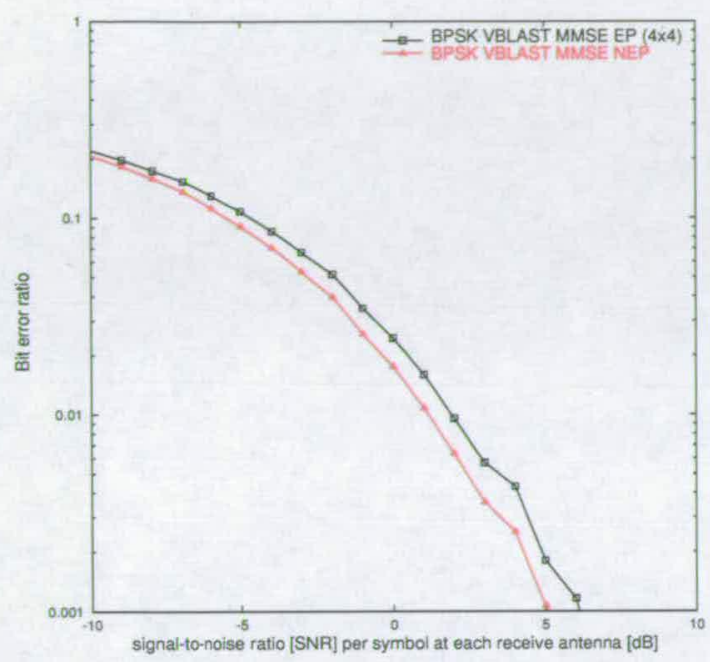

(a)

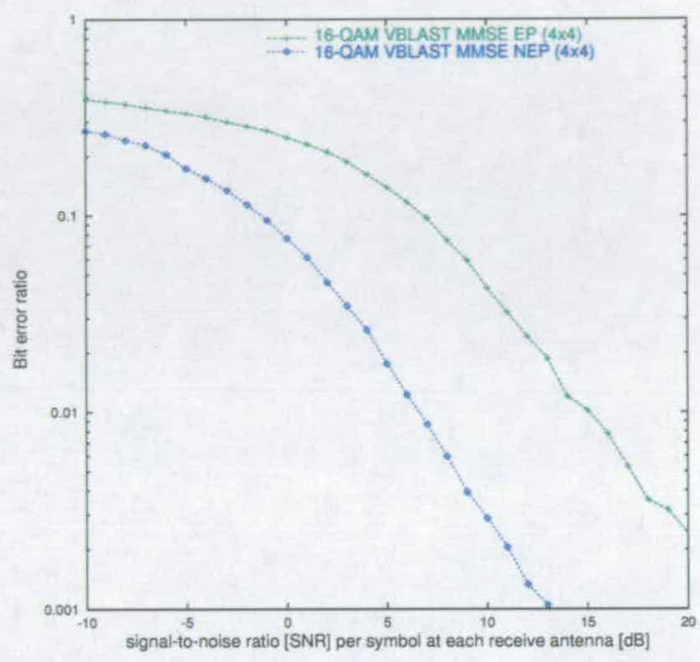

(b)

Figure 3.13: Effect of Error Propagation on MIMO (4,4): (a) BPSK modulation - MMSE/VBLAST MMSE detector, (b) 16-QAM modulation - MMSE/VBLAST MMSE detector

\subsubsection{Spatial Fading Complex Correlation}

In this section the effect of spatial fading complex correlation on different MIMO systems is investigated. First, we look at the effect of correlation in a $(4,4)$ MIMO system under 16-QAM modulation and VBLAST MMSE detection. Figure 3.15(a) shows the performance of a MIMO $(4,4)$ system under different degrees of correlation. It is clear that any increase in the degree of the system spatial correlation leads to degradation in the system's performance. This is due to the fact that correlation between the antenna array elements results in a channel matrix with low rank [25]. The channel $\boldsymbol{H}$ may offer $K$ parallel subchannels with different mean gains, with $K=\operatorname{Rank}\left(\boldsymbol{R} \leq \min \left(N_{T}, N_{R}\right)\right)$, where the function $\operatorname{Rank}($.$) and \min ($.$) return the rank of the$ matrix and the minimum value of the arguments, respectively. $\boldsymbol{R}$ is the instantaneous correlation matrix $\boldsymbol{R}=\boldsymbol{H} \boldsymbol{H}^{H}$. When the rank $K$ of the channel matrix is low(high correlation), then less subchannels are available to the system, which results in degradation of the performance of the system. Another point that is worth mentioning is that high correlation only at the one end of the communication (either at receiver or at transmitter) has nearly the same impact on the BER performance, as in the case when both ends of the communication highly correlated. In Figure 3.15(b) we present some results on the the correlation effect for two different detectors (MMSE and VBLAST MMSE). The simulation is of a $(4,4)$ MIMO system employing 


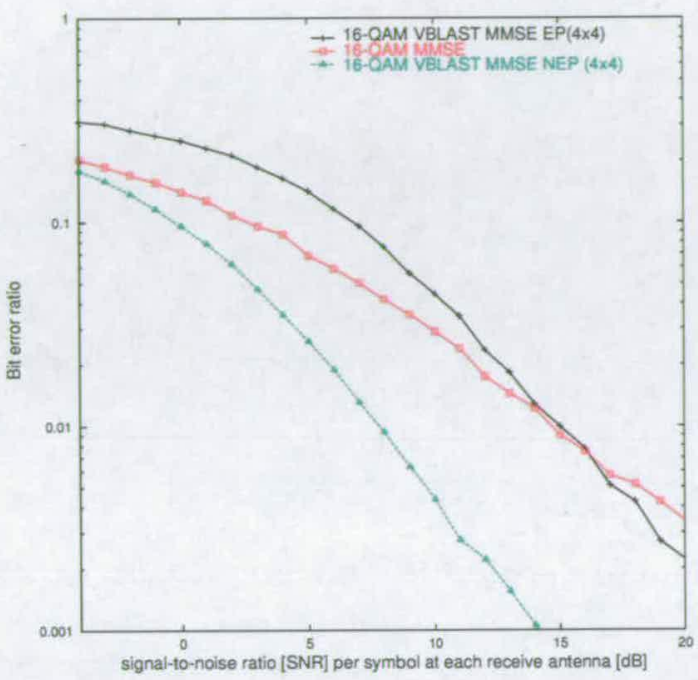

Figure 3.14: Effect of Error Propagation on a (4,4) MIMO 16-QAM under two different detection techniques: MMSE/VBLAST MMSE

16-QAM modulation. From the graph, it is obvious that for decorrelated channels (no correlation or 0.3 correlation) the VBLAST MMSE detector gives a high SNR gain. Specifically, at the BER value of 0.001 the SNR gain due to VBLAST MMSE detector is more than $9 \mathrm{~dB}$ over MMSE detector, for the case of completely uncorrelated channels. Similar values hold for the decorrelated case ( 0.3 correlation). On the other hand, in the case of high correlation ( 0.96 correlation at the BS and 0.3 at the MS) the VBLAST MMSE detector performs no better than MMSE for system SNR values less than 10dB. For larger than 10dB SNR values VBLAST MMSE shows some improvement. An explanation for this behaviour of the VBLAST detection is, that the VBLAST technique is exploiting the spatial multiplexing in a MIMO multipath environment, but as we have shown before, in a high correlated environment the rank of the channel matrix is low. Low rank channels do not support spatial multiplexing effectively.

Figure 3.16 depicts the results in BER for the real measured examples (correlation coefficient as presented in matrices $3.37,3.36$ ). The measured system (as well as the simulated one) is a $(4,4)$ MIMO one employing BPSK modulation.We compare the results of the measured correlation matrices with the results of produced by randomly created correlation coefficients, whose average magnitude is similar to the measured coefficients magnitude. The phases of the randomly created complex correlation coefficient are random but not independent of each other, since they are connected through the scattering environment. The graph shows a good convergence between the measured and simulated spatial complex correlation matrices.

Finally, Figure 3.17 shows the effect of spatial fading correlation ( 0.96 at the BS and 0.1 at the 


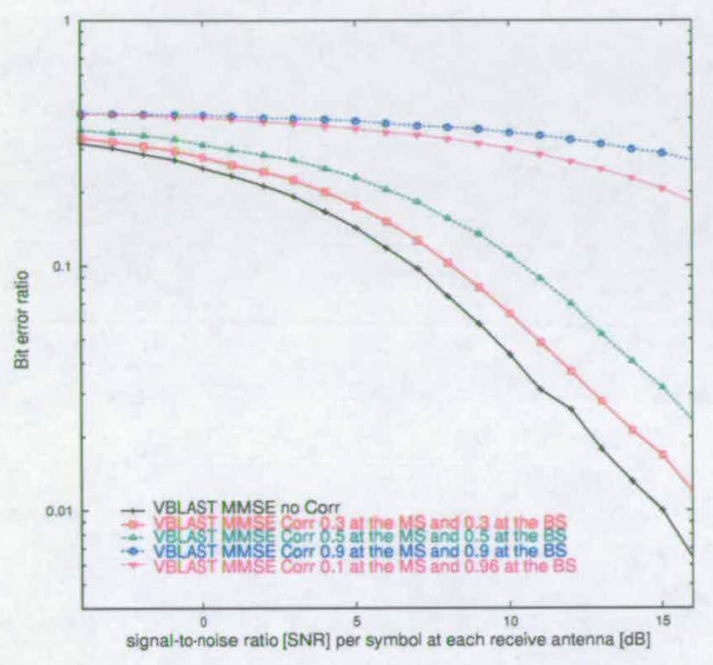

(a)

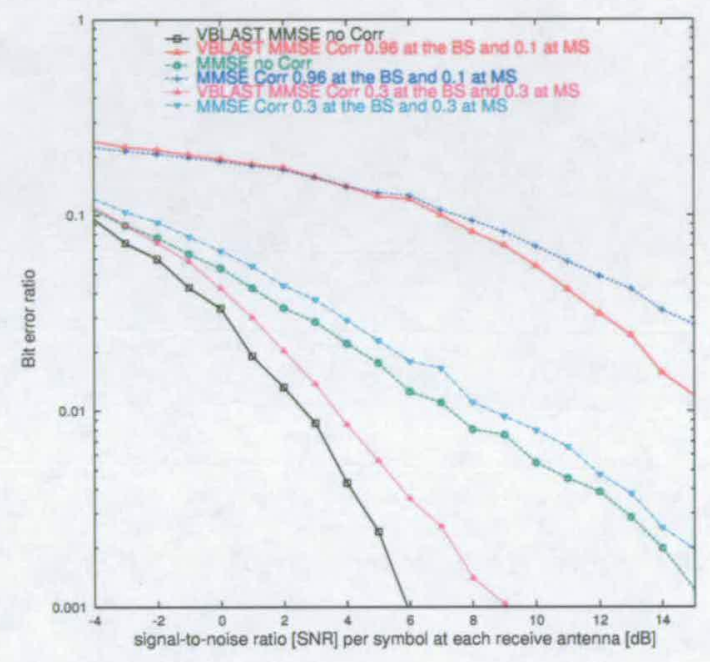

(b)

Figure 3.15: Effect of spatial fading complex correlation on a (4,4) MIMO system: (a) 16-QAM modulation - VBLAST MMSE detector, (b) BPSK modulation - MMSE/VBLAST MMSE detector

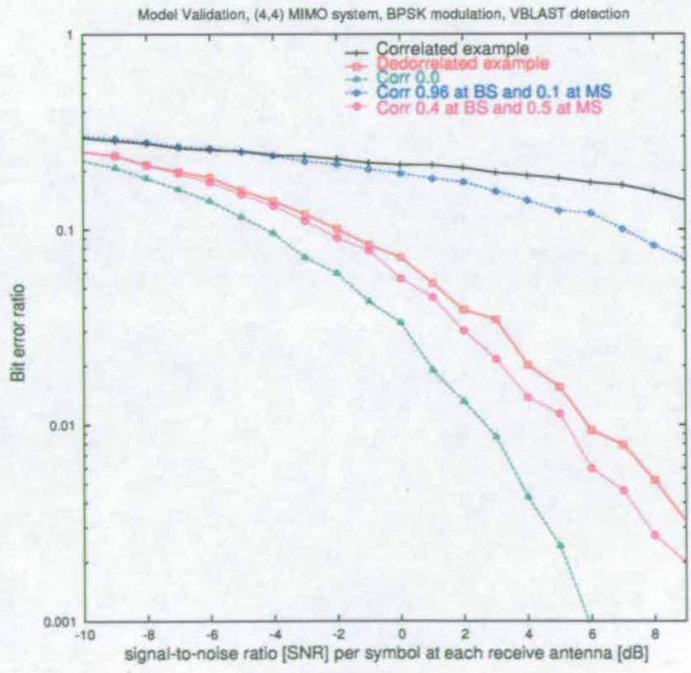

Figure 3.16: Comparison of the results of the measured correlation matrices with the results of the simulated correlation matrices 


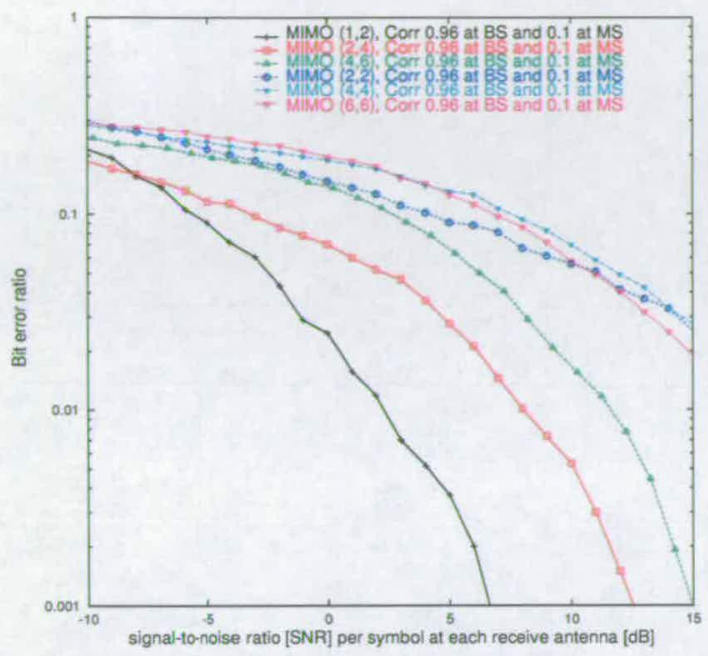

(a)

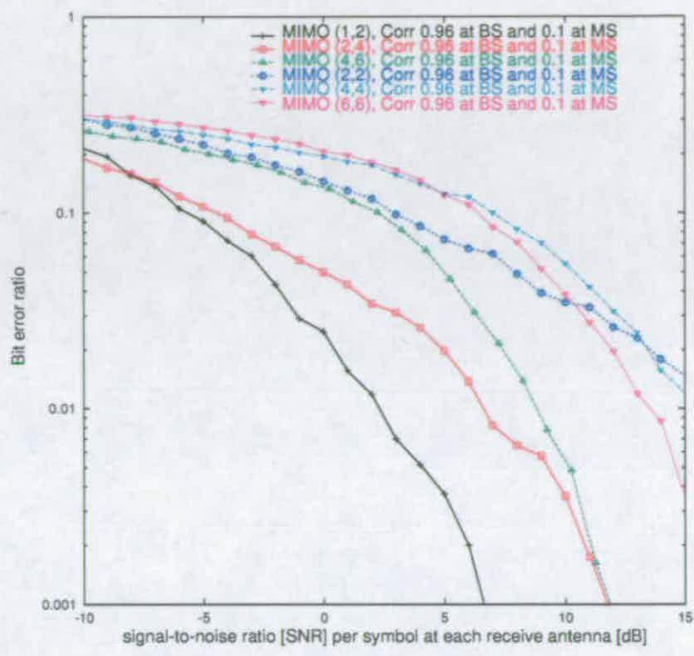

(b)

Figure 3.17: Effect of spatial fading complex correlation on BPSK MIMO systems with different sizes of antenna arrays at transmitter and receiver: (a) MMSE detector, (a) VBLAST MMSE detector

MS) on MIMO systems of different number of transmit and receive antennas. In Figure 3.17(a), we present the results for MIMO systems where MMSE detection and BPSK modulation have been employed. In this case, the $(1,2)$ MIMO system gives lower values of BER of any other MIMO system for the same SNR. The physical meaning of correlation is that the instantaneous channels of the various transmit antennas become quasi-identical due to high correlation (at the transmitter). This phenomenon makes the MIMO system equivalent to a SIMO system, and any advantages due to spatial multiplexing disappear. When the transmit antennas are less than the receive antennas (MIMOs $(1,2),(2,4),(4,6))$, the increase of the transmit antennas leads to degradation of the system BER, eg. at the value of BER equal to 0.001 there is a $7 \mathrm{~dB}$ SNR loss in a MIMO $(2,4)$ compared to a MIMO $(1,2)$ and at the same value of BER there is a $2 \mathrm{~dB}$ loss in a MIMO $(4,6)$ compared to a MIMO $(2,4)$. The presence of correlation cancels the advantage of linear detection. The MMSE detector is a linear detector, that forms the received signals estimates by linearly combining the received signals samples. These received signals, under the correlation effect tend not to be linearly independent any more, but any linear transformation of a set of linearly dependent vectors leads to null vector space. In linear algebra [135], a vector space $V$ has dimension $n$ if it contains a linearly independent set of $n$ vectors. In the case of high correlated channel the received signals are linearly dependent. So, any received 
signal can be expressed as linear combinations of the others. The physical interpretation is that high correlation reduces the available spatial parallel datastreams that can be multiplexed. This phenomenon combined with the fact that the transmit antennas share the same system SNR, are responsible for the results depicted on the graph.

In 3.17(b), we investigate the VBLAST MMSE detector under high spatial correlation and for different sizes of antenna arrays at transmitter and receiver. The graph is quite similar to the previous graph (Figure 3.17(a)). Specifically, at the BER value equal to 0.001 there is a $6 \mathrm{~dB}$ SNR loss in a MIMO $(2,4)$ compared to a MIMO $(1,2)$. At larger SNR $(>7 \mathrm{~dB})$, the VBLAST MMSE detection becomes better for a MIMO $(4,6)$ system.

\subsection{Conclusions}

In this chapter, we aimed to investigate the physical channel performance. We split down the system model into three main parts (transmitter, physical channel and receiver) and analyzed each part separately. Taking into account the most popular MIMO channel models, we chose the channel models that are used throughout this work. We described and investigated the structure of our MIMO physical channel model and characterized the performance of this channel. Detection algorithms (VBLAST MMSE, MMSE) for single-user wireless communication using multiple antennas at both the transmitter and receiver in a stochastic MIMO radio channel model were compared for different degrees of correlation between the antenna array elements. The effect of error propagation was also investigated. All the simulation scenarios have been implemented for two modulation schemes (BPSK and 16-QAM). The stochastic MIMO channel model was simulated using both a measured [107] and theoretical fading correlation model. The simulation results show an improvement on the system performance (in terms of BER) due to VBLAST MMSE detection over MMSE detection. Note, that in the case of error propagation and 16-QAM modulation (Figure 3.14), MMSE detection is proven to be more efficient detection method than VBLAST MMSE. Also, with reference to Figure $3.15-3.16$ it is obvious that the spatial fading complex correlation between antenna arrays leads to a significant degradation of the system performance.

The results presented in this chapter mirror those already published in the literature. However, in the next chapter, we will investigate the the performance of different packet scheduling techniques in a multi-user environment, we will explore performance trade offs that have not 
previously been investigated in the literature. 


\section{Chapter 4 Exploiting Multiuser Diversity for MIMO Cellular Systems using RRS Packet Scheduling and the VBLAST Receiver}

In the previous chapter we investigated the impact on performance of the physical channel using MIMO techniques. In this chapter, we study the use of packet scheduling in MIMO multiuser wireless systems, and we present some new packet scheduling schemes based on the round robin scheme (RRS) [39]. Specifically, in section 4.1 we introduce packet scheduling and the multiuser diversity concept. In section 4.2 , we present the system and channel models for a MIMO cellular system. In section 4.3, we describe briefly the conventional RRS scheme and the AA-RRS (antenna-assisted round robin scheduling) scheme, and the system capacity expressions for these schemes. In section 4.4 we propose the VBLAST AA-RRS (VBLAST antenna-assisted round robin scheduling), best user, AA MMSE (antenna-assisted MMSE) fair and AA VBLAST (antenna-assisted VBLAST) fair scheduling schemes, and we present the capacity expressions for these schemes. In section 4.5 , the simulation model and results are presented to compare the performance metrics of the proposed and existing packet scheduling schemes, and to investigate the effects of spatial correlation. Finally, conclusions are drawn in section 4.6 .

\subsection{Introduction to Scheduling}

Multiple-input multiple-output (MIMO) systems have been proven to be efficient for achieving high capacity over wireless links [27]. In MIMO systems based on spatial multiplexing, each data stream is split into multiple substreams, and each of these substreams is transmitted through one of the transmit antennas [131]. In multiuser environments, independence of fading among users, called multiuser diversity [136], can be exploited to increase the system capacity. 
Motivated by the information theoretic results in [80] and [37], one approach to increase the throughput of multiuser systems is to use multiuser diversity to take advantage of the independence of the fading statistics of the different users [79]. To benefit from multiuser diversity in cellular packet transmission systems, a packet scheduler should preferentially allocate radio resources to users in good channel conditions. Two critical targets of packet scheduling are to maximize the system capacity (throughput) and to offer fairness among users. In [136], a channel state dependent scheduling scheme maximizes the system capacity through the use of multiuser diversity. Specifically, each spatial channel is allocated to a user with the best channel condition for each time slot. Therefore, some users in adverse channel conditions may not be served, causing an unfaimess problem. In [39], the round robin scheduling (RRS) scheme has been studied for MIMO cellular systems. The RRS scheme operates in a cyclic fashion regardless of the channel conditions, and thus achieves faimess among users [39]. The RRS however, does not use multiuser diversity, resulting in the same capacity as a single user system. In order to exploit the multiuser diversity and at the same time maintain fairness across the users, two other scheduling schemes have been proposed in [137] and [138]. In [137] the antenna-assisted round robin scheduling (AA-RRS) scheme has been proposed. The AA-RRS is an improved RRS scheme that exploits multiple antennas to achieve a diversity effect from multiple users. The opportunistic scheduling [138] uses multiple antennas and a proportional fair scheduler (PF). The PF scheduler assigns a user for transmission when its instantaneous channel capacity is high relative to its average channel condition. As such, the benefit of multiuser diversity can be exploited and fairness can be maintained (the PF scheduler will be discussed in detail in the next chapter). This chapter proposes a variation of AA-RRS scheme, where VBLAST detection is applied to each receiver. This novel technique is shown to improve significantly the system capacity. Also, note that throughout this chapter we assume a packet based wireless communication system where the transmitted signal is partitioned into long bursts.

\subsection{System Model}

A simple model for the downlink of a single cell MIMO cellular system is illustrated in Figure 4.1. There is a single base transceiver station (BS) communicating with $K$ mobile stations (MSs). The BS has $N_{T}$ transmit antennas while each user has $N_{R}$ receive antennas. A queue of packets is stored at the BS for each of the $K$ users. The BS serves the users in a time division fashion, and the $K$ users are distributed uniformly over the circular cell area (Figure 4.1). At 


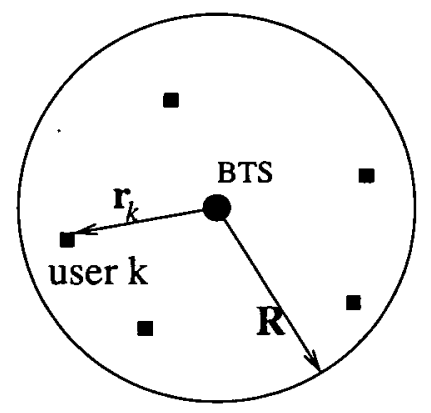

Figure 4.1: Aerial view of the cell.

the BS, the transmit power is equally divided into transmit antennas, and the receiver of each user estimates transmit symbols destined for the user using the VBLAST detector [110] with minimum mean-squared error (MMSE) nulling [126] [137].

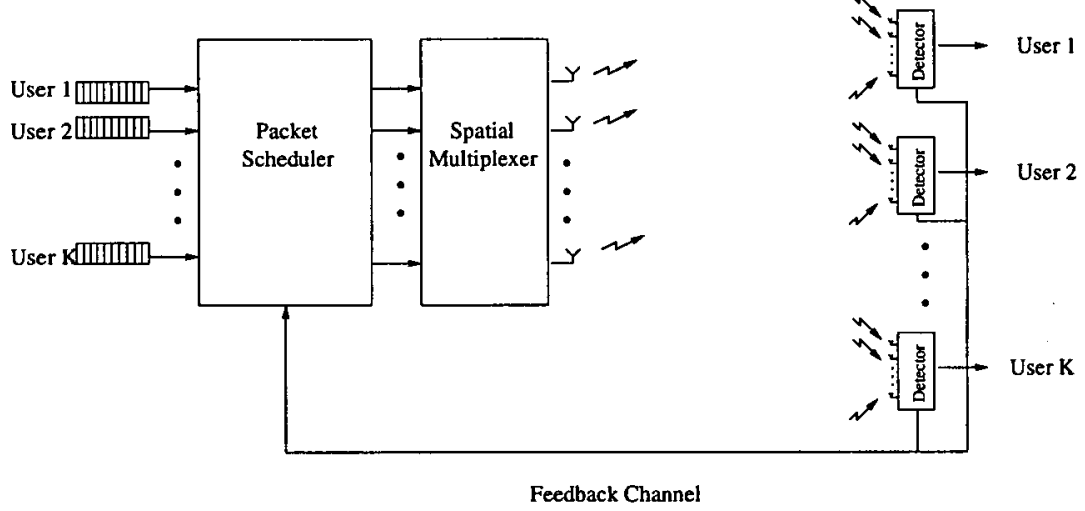

Figure 4.2: Downlink MIMO cellular system.

In such a MIMO system, each transmit antenna creates a spatial channel [136], resulting in a total of $N_{T}$ spatial channels for each time slot. The receiver estimates the post-detection signal-to-interference-plus-noise ratio (SINR) for each transmit antenna, and passes the SINR information to the BS, as shown in Figure 4.2. The packet scheduler at the BS determines which packet to transmit through which transmit antenna. The transmit signals from the BS are assumed to experience path loss, log-normal shadow fading and multipath fading. In our work we adopt the block-fading model [139], as it provides a first-order approximation to the continuously time varying channel, and it is simple enough to be mathematically tractable [140]. The channel is assumed to be fixed during a time slot, and to vary independently over time slots. 
The channel matrix $\boldsymbol{H}_{k}(t)$ between the BS and the user $k$ for a time slot $t$ may be expressed as

$$
\boldsymbol{H}_{k}(t)=\sqrt{S N R_{0}\left(r_{k} / R\right)^{-a_{D L}} 10^{S_{k}(t) / 10}} G_{k}(t)
$$

where $S N R_{0}$ denotes the median SNR at the cell boundary, $r_{k}$ is the distance between the BS and the user $k, a_{D L}$ is the path loss exponent, and $S_{k}(t)$ is a real Gaussian random variable with zero mean and variance of $\sigma_{S}^{2}$. An $N_{R} \times N_{T}$ matrix $G_{k}(t)$ represents Rayleigh-distributed multipath fading. In the simulation we assume different degrees of spatial fading correlation. The elements of $\boldsymbol{G}_{k}(t)$ are independent complex Gaussian random variables with zero mean and unit variance, in the case of uncorrelated channel. In the case of correlation, the elements of $G_{k}(t)$ are determined using the model described in chapter 3. The values we consider for $S N R_{0}$ (median SNR at the cell boundary) are $-5 \mathrm{~dB}, 0 \mathrm{~dB}$, and $10 \mathrm{~dB}$. The values of $-5 \mathrm{~dB}$ and $0 \mathrm{~dB}$ represent the median SNR at the cell boundary for more realistic cases of a cellular network. On the other hand the value of $10 \mathrm{~dB}$ represent the case of a small cell - high data rate scenario.

\subsubsection{Physical Channel Characteristics}

Geographical and physical factors like building structures, hills, weather conditions and generally any physical obstacle, can affect the properties of a wireless channel. Many models have been developed to describe different aspects of radio channel characteristics as discussed in chapter 3. The model we have chosen (equation (4.1)) takes into consideration three basic physical aspects.

First, the term $\left(r_{k} / R\right)^{-a_{D L}}$ represents the path loss. In free space, this loss follows the inverse square law, but in many situations, the signal does not go through free space. The earth's surface acting as a large reflecting object changes the model substantially. In urban cellular systems, the path loss exponent $a_{D L}$ is usually between 3-5 [40]. This higher exponent path loss model has advantages and disadvantages. The drawback is that the transmission power has to be higher for a given coverage area and the power levels of near and far users will be very different, causing increased interference for low power users. The advantage is that the cell coverage is sharper and co-channel interference from other clusters is small. The second term of equation (4.1), $\left(10^{S_{k}(t) / 10}\right)$, represents the shadowing effect. Shadowing includes the effect of large obstacles (hills, buildings etc) and causes a variation around the mean power that is 
modeled by path loss. The standard deviation of the Gaussian term is typically $6-8 \mathrm{~dB}$. It will be higher in urban settings and indoor systems, but lower in rural areas. Finally, the last term in channel equation (4.1) expresses multipath. The local scatterers, located at the receiver or at the transmitter, give rise to a large number of signal paths, that are picked up by the transmitter or the receiver respectively. This phenomenon is called multipath. Signal multipath occurs when the transmitted signal arrives at the receiver via multiple propagation paths at different delays [41]. It generally results in intersymbol interference (ISI) in a digital communication system. Moreover, the signal components arriving via different propagation paths may add destructively, resulting in a phenomenon called signal fading [41].

\subsection{Packet Scheduling and System Capacity}

In this section, we describe briefly the conventional RRS scheme [39] and the newer AA-RRS scheme [137], and then we present our proposed scheduling scheme based on AA-RRS.

\subsubsection{Round Robin Scheduling (RRS)}

First, in the RRS [39], one among $K$ users is selected at each time slot in a round robin fashion, and all the $N_{T}$ transmit antennas are assigned to the selected user for the time slot. Round robin algorithm has a computational complexity of $\mathrm{O}(1)$. When the $k$ th user is selected at time slot $t$, the system capacity $C_{R R S}(t)$ for that time slot may be expressed as [141]

$$
C_{R R S}(t)=\sum_{n=1}^{N_{T}} \log _{2}\left(1+\gamma_{k, n}(t)\right)
$$

where $\gamma_{k, n}(t)$ denotes the post-detection SINR for the channel corresponding to the $n$th transmit antenna and the $k$ th user. The post-detection SINR is defined as the SINR of a transmit symbol after MMSE nulling, and it may be expressed as [141]

$$
\gamma_{k, n}(t)=\frac{P_{R}\left|\left[W_{k}(t) H_{k}(t)\right]_{n n}\right|^{2}}{N_{0}^{2} N_{T} \sum_{m=1}^{N_{T}}\left|\left[W_{k}(t)\right]_{n m}\right|^{2}+P_{R} \sum_{m=1, m \neq n}^{N_{T}}\left|\left[W_{k}(t) H_{k}(t)\right]_{n} m\right|^{2}}
$$

where $P_{R}$ is the total received signal power, and $N_{0}^{2}$ is the noise power per received antenna. The nulling weight matrix $W_{k}(t)$ for the MMSE filter is given as [141] 


$$
W_{k}(t)=\boldsymbol{H}_{k}^{H}(t)\left(\boldsymbol{H}_{k}(t) \boldsymbol{H}_{k}^{H}(t)+\left(N_{0}^{2} N_{T} / P_{R}\right) I_{N_{R}}\right)^{-1}
$$

where $(\bullet)^{H}$ denotes the conjugate transpose, and $I_{N_{R}}$ is the $N_{R} \times N_{T}$ identity matrix. It is obvious that the average cell throughput does not depend on the number of users, and thus the RRS scheme provides the same system capacity as a single user MIMO system.

\subsubsection{Antenna-Assisted Round Robin Scheduling (AA-RRS)}

The AA-RRS scheme [137] exploits the multiple spatial channels of MIMO systems to achieve a diversity effect from multiple users. Specifically, $K$ users are initially listed in such a way that each user is identified with a unique user index $k(k=\{1,2, \ldots, K\})$. At each time slot, $N_{T}$ users are selected from $K$ users, and these users form a scheduled user group (SUG) for the time slot. The selection of users for an SUG is made in a round robin fashion. Once an SUG is formed, the spatial channels or transmit antennas should be assigned to users in the SUG. The post-detection SINR information determines the mapping. The post-detection SINR is calculated at the receiver, and then is fed back from each user to the BS, as in Figure 4.2. A mapping between transmit antennas and users may be represented as a sequence $\left(k_{1}, k_{2}, \ldots, k_{N_{T}}\right)$, with $k_{n}$ denoting the user index assigned to the $n$th transmit antenna. For a given sequence of $k_{n}$ 's the system capacity for the time slot $t$ may be calculated as [137]

$$
\left.C_{A A-R R S}\left(t \mid\left(k_{1}, k_{2}, \ldots, k_{N_{T}}\right)\right)=\sum_{n=1}^{N_{T}} \log _{2}\left(1+\gamma_{k_{n}, n}\right)\right)
$$

where $\gamma_{k_{n}, n}$ denotes the post detection SINR for the channel between the $n$th transmit antenna and the $k_{n} t h$ user, as expressed in [141]

$$
\gamma_{k, n}(t)=\frac{P_{R}\left|\left[W_{k}(t) H_{k}(t)\right]_{n n}\right|^{2}}{N_{0}^{2} N_{T} \sum_{m=1}^{N_{T}}\left|\left[W_{k}(t)\right]_{n m}\right|^{2}+P_{R} \sum_{m=1, m \neq n}^{N_{T}}\left|\left[W_{k}(t) H_{k}(t)\right]_{n} m\right|^{2}}
$$

The sequence of $k_{n}$ 's that maximizes the system capacity may be found using an exhaustive search (EXS) over all possible sequences, and the corresponding capacity can be expressed as [137]: 


$$
C_{A A-R R S, E X S}(t)=\underset{\left\{\left(k_{1}, k_{2}, \ldots, k_{N_{T}}\right) \mid k_{i} \neq k_{j}, \text { if } i \neq j\right\}}{\arg \max } C_{A A-R R S}\left(t \mid\left(k_{1}, k_{2}, \ldots, k_{N_{T}}\right)\right)
$$

Note, that the demand $\left\{k_{i} \neq k_{j}\right.$, if $\left.i \neq j\right\}$ ensures that user will not be allocated to multiple antennas.

The computational complexity of AA-RRS scheme is studied as a function of the number of active users $K$, and the number of transmit antennas $N_{T}$ (we assume that $N_{T}=N_{R}$ ). The algorithm first performs $N_{T}$ iterations of the MMSE algorithm to calculate $\gamma_{k_{n}, n}$ between the $N_{T}$ transmit antennas and $N_{T}$ users of the SUG. The MMSE algorithm has a complexity order of $\mathrm{O}\left(N_{T}^{3}\right)$. After the calculation of the post-detection SNR values, the algorithm requires $N_{T}$ ! comparisons to find the mapping sequence that maximizes the system capacity. Summarizing, the computational complexity of the AA-RRS scheme is $\mathrm{O}\left(N_{T}^{4}+N_{T} !\right)$. Also note that in the implementation of this algorithm we have to add extra information in every packet header concerning the user-transmit antenna allocation. This information will be updated every time slot.

\subsection{Proposed Scheduling Schemes}

In this section we introduce four scheduling algorithms. The proposed algorithms are based on the AA-RRS scheme [137]. The algorithms presented are, the VBLAST AA-RRS scheme (section 4.4.1), the best user scheme (section 4.4.3), the AA MMSE fair scheme (section 4.4.4) and the AA VBLAST fair scheme (section 4.4.5). In sections 4.4.3.1 and 4.4.3.2 we present two different implementations of the best user scheme, the AA-RRS and VBLAST AA-RRS respectively.

\subsubsection{VBLAST AA-RRS Feasible Data Rates Algorithm}

The proposed VBLAST AA-RRS scheme is based on the AA-RRS scheme [137]. The basic difference is the use of VBLAST detectors at each receiver. VBLAST detection [131], as discussed in Chapter 3, is an improved technique for exploiting spatial multiplexing in MIMO multipath environment. The main idea of using VBLAST detection is the feature of the symbol cancellation. Using symbol cancellation, interference from already-detected components of the transmitted signal are subtracted out from the received signal vector, resulting in a modified 
receiver vector in which fewer interferers are present. When nulling with symbol cancellation are used together (VBLAST) the system performs better than in the case of pure nulling [131]. The post-detection SINR $\left(\gamma_{k, n}(t)\right)$ for the channel corresponding to the $n t h$ transmit antenna and the $k$ th user, is defined as the SINR of a transmit symbol after symbol cancellation and MMSE nulling, and it may be expressed as

$$
\gamma_{k, n}(t)=\frac{P_{R}\left|\left[W_{k}(t) H_{k}(t)\right]_{n n}\right|^{2}}{N_{0}^{2} N_{T} \sum_{m=1}^{N_{T}}\left|\left[W_{k}(t)\right]_{n m}\right|^{2}+P_{R} \sum_{m=1, m \neq n, m \notin D_{k, n}(t)}^{N_{T}}\left|\left[W_{k}(t) \boldsymbol{H}_{k}(t)\right]_{n m}\right|^{2}}
$$

where $D_{k, n}(t)$ denotes a vector, whose elements are the transmit antennas that have been detected by user $k$ before the detection of transmit antenna $n$.

$$
\boldsymbol{D}_{k, n}(t)=\left\{d_{k, 1}(t), d_{k, 2}(t), \cdots, d_{k, l}(t)\right\}
$$

where $d_{k, i}(t)$ denotes the index of the detected antenna from user $k$ during the $i$ th iteration of the VBLAST detection algorithm. If the transmit antenna $n$ is detected by user $k$ during the $(l+1)$ th iteration of the VBLAST detection algorithm, then last element of the vector $D_{k, n}(t)$ is the element $d_{k, l}(t)$, which is the index of the transmit antenna that has been detected by user $k$ during the $l$ th iteration of the VBLAST detection algorithm. The demand $\{m \neq$ $\left.n, m \notin D_{k, n}(t)\right\}$ ensures that the interference from already detected components of transmitted signal are subtracted out from the received signal vector, resulting in a higher post-detection SINR. The nulling weight matrix $W_{k}(t)$ for the MMSE detector is given as in equation (4.4). The modified receiver vector gives higher post-detection SINRs (equation (4.8)) and therefore higher system capacity.

To describe the algorithm analytically, consider $K$ active users that are initially listed in such a way that each user is identified with a unique user index $k(k=1,2, \ldots, K)$. At each time slot, $N_{T}$ users are selected from $K$ users, and these users form a scheduled user group (SUG) at the time slot. The selection of users for an SUG is made in a round robin fashion. For example, in the case of $K=6$ and $N_{T}=4$, the SUGs for subsequent time slots are $\{1,2,3,4\},\{5$, $6,1,2\},\{3,4,5,6\}$, and so forth, where the numbers denote user indices. In the special case of $K<N_{T}$, users are allowed to participate in the SUG more than once in a time slot. Once 


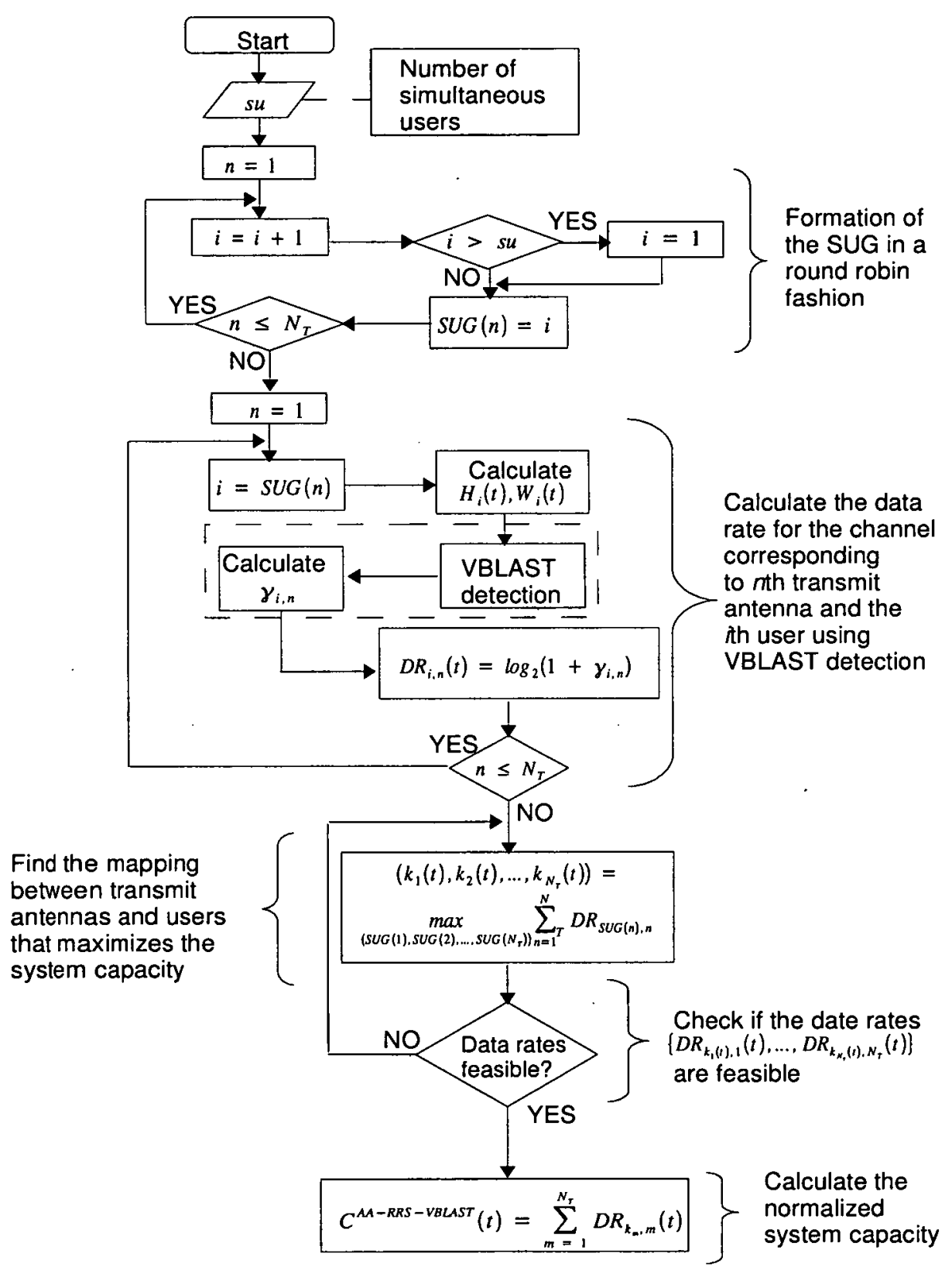

Figure 4.3: AA-RRS-VBLAST algorithm 
an SUG is formed, the spatial channels or transmit antennas should be assigned to users in the SUG. To guarantee fair channel access as in the RRS scheme, we restrict each scheduled user to use only one transmit antenna. This means that every user can use one spatial channel every $K / N_{T}$ time slots, or equivalently $N_{T}$ channels every $K$ time slots, as in RRS scheme. There are $N_{T}$ ! possible one-to-one mappings between $N_{T}$ scheduled users and $N_{T}$ transmit antennas, and an appropriate choice of mapping may realize a strong diversity effect. It is possible to integrate all the possible one-to-one mappings into a matrix $\boldsymbol{P}(t)$ of size $N_{T} ! \times N_{T}$.

$$
\boldsymbol{P}(t)=\left[\begin{array}{cccc}
p_{1,1}(t) & p_{1,2}(t) & \cdots & p_{1, N_{T}}(t) \\
p_{2,1}(t) & p_{2,2}(t) & \cdots & p_{2, N_{T}}(t) \\
\vdots & \vdots & \ddots & \vdots \\
p_{N_{T} !, 1}(t) & p_{N_{T} !, 2}(t) & \cdots & p_{N_{T} !, N_{T}}
\end{array}\right]_{N_{T} ! \times N_{T}}
$$

where $p_{m, n}(t)$ denotes the user $k$ that is allocated to $n$th transmit antenna according to the $m$ th mapping sequence.

We utilize the post-detection SINR information for determining an effective mapping. The post-detection SINR is calculated at the receiver, and then is fed back from each user to the BS, as in Figure 4.2. The post-detection SINR information for the $N_{T}$ users in the SUG at time slot $t$ may be integrated into a matrix as

$$
\boldsymbol{\Gamma}_{s i n r}(t)=\left[\begin{array}{cccc}
\gamma_{1,1}(t) & \gamma_{1,2}(t) & \cdots & \gamma_{1, N_{T}}(t) \\
\gamma_{2,1}(t) & \gamma_{2,2}(t) & \cdots & \gamma_{2, N_{T}}(t) \\
\vdots & \vdots & \ddots & \vdots \\
\gamma_{N_{T}, 1}(t) & \gamma_{N_{T}, 2}(t) & \cdots & \gamma_{N_{T}, N_{T}}
\end{array}\right]_{N_{T} \times N_{T}}, K \geq N_{T}
$$

where $\gamma_{k, n}(t)$ denotes the post-detection SINR for the channel between the $n$th transmit antenna and the $k$ th user in the SUG, as expressed in equation (4.8). A mapping between transmit antennas and users may be represented as a sequence $\left(k_{1}, k_{2}, \ldots, k_{N_{T}}\right)$, with $k_{n}$ denoting the user index assigned to the $n$th transmit antenna. For a given sequence of $k_{n}^{\prime}$ 's the system capacity for the time slot $t$ may be calculated as :

$$
C_{A A-R R S-V B L A S T}\left(t \mid\left(k_{1}, k_{2}, \ldots, k_{N_{T}}\right)\right)=\sum_{n=1}^{N_{T}} \log _{2}\left(1+\gamma_{k_{n}, n}\right)
$$


where $\gamma_{k_{n}, n}$ denotes the post detection SINR for the channel of the $n t h$ transmit antenna and the $k_{n} t h$ user, as expressed in equation (4.8). The sequence of $k_{n}$ 's that maximizes the system capacity may be found using an exhaustive search (EXS) over all possible sequences, and the corresponding capacity can be expressed as:

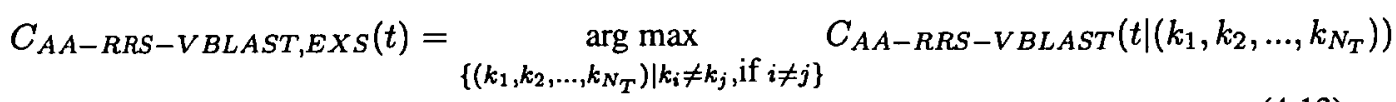

Specifically, the flowchart of the algorithm is shown in Figure 4.3. The flowchart of the VBLAST detection algorithm, that is used by VBLAST AA-RRS algorithm (Figure 4.3), is illustrated in Figure 4.4. The pseudocodes of the VBLAST AA-RRS algorithm and the VBLAST detection are presented in appendix A (Figures A.1 and A.2 respectively).

The computational complexity of the VBLAST AA-RRS scheme is studied as a function of the number of active users $K$, and the number of transmit antennas $N_{T}$ (we assume that $N_{T}=\dot{N}_{R}$ ). The algorithm first performs $N_{T}$ iterations of the VBLAST algorithm to calculate $\gamma_{k_{n}, n}$ between the $N_{T}$ transmit antennas and $N_{T}$ users of the SUG. The VBLAST algorithm has a complexity order of $\mathrm{O}\left(N_{T}^{4}\right)$. After the calculation of the post-detection SNR values, the algorithm requires $N_{T}$ ! comparisons to find the mapping sequence that maximizes the system capacity. Summarizing, the computational complexity of the VBLAST AA-RRS scheme is $\mathrm{O}\left(N_{T}^{5}+N_{T}\right)$.

\subsubsection{Feásible Data Rates}

One constraint for VBLAST AA-RRS is that the data rates of the new allocation scheme should be feasible. In short, the assumed data rates have to be smaller than the capacities of the wireless links. In the VBLAST algorithm, a signal must be detected successfully for it to be subtractively cancelled. This leads to additional capacity constraints as compared to the MMSE detector. If these constraints are not met, the VBLAST detectors would not be able to detect the transmitted signals.

As an example, we consider the scenario of a single cell MIMO cellular system. As depicted in Figure 4.5, the base station is equipped with a uniform linear array with 2 antenna elements and each mobile user is equipped with a uniform linear array with 2 antenna elements. After 


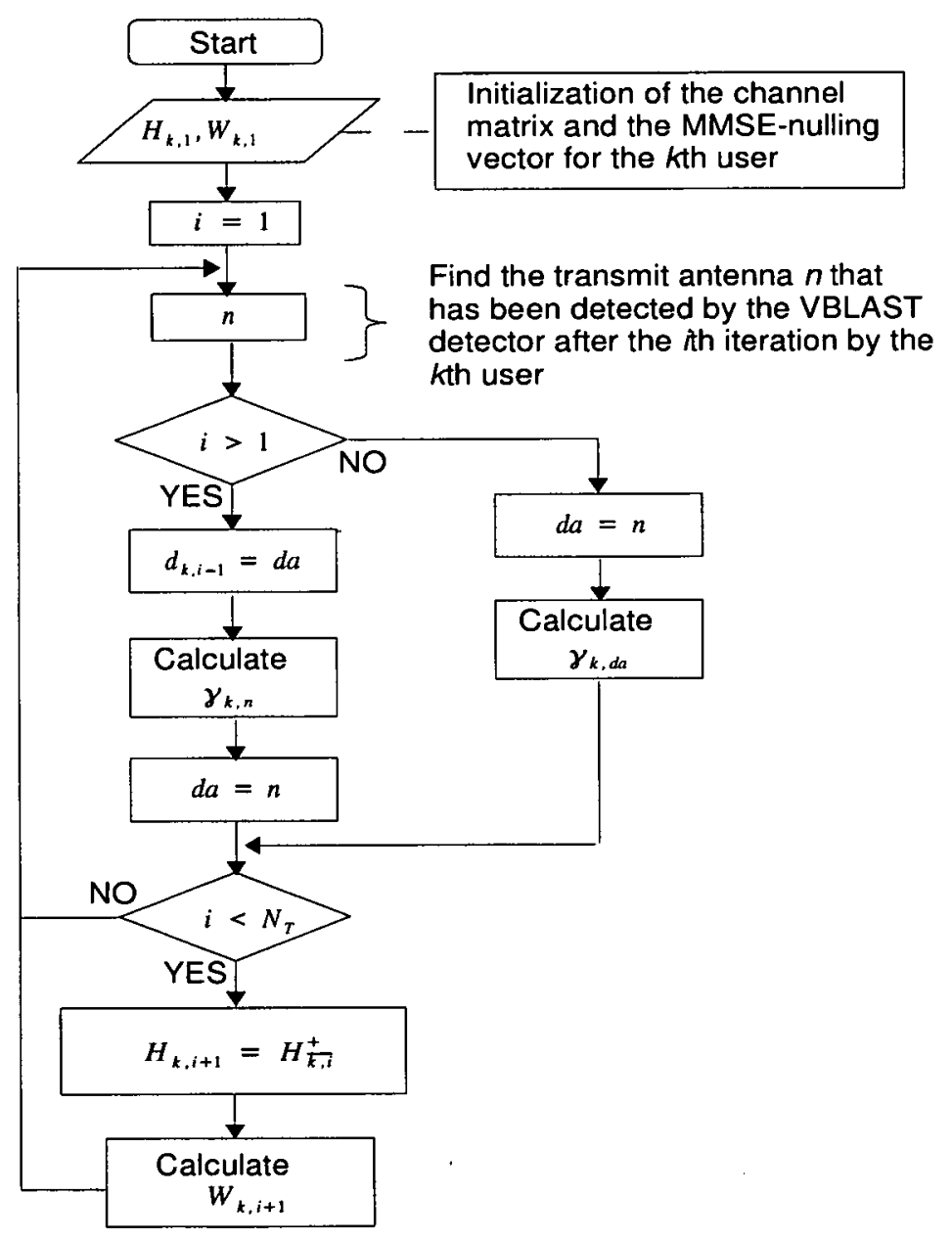

Figure 4.4: VBLAST detection 

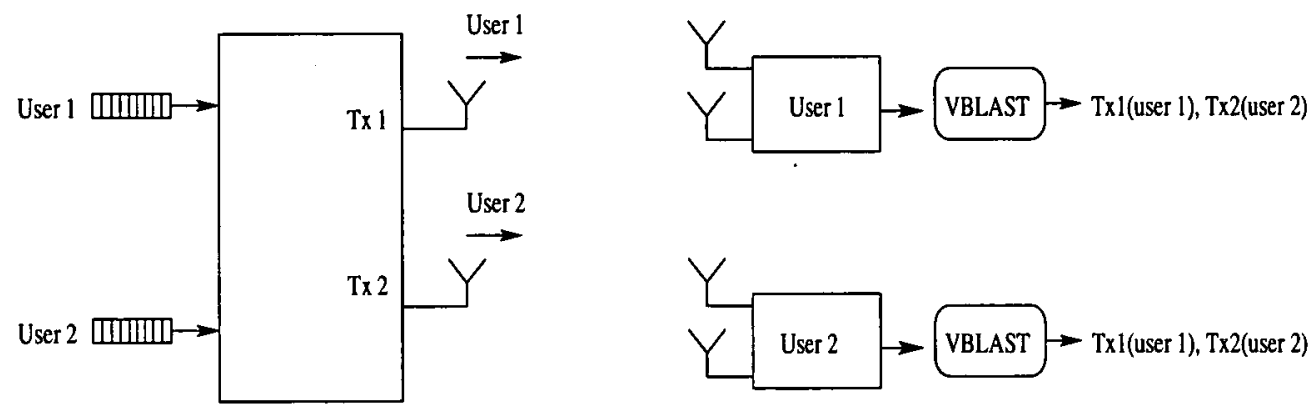

Figure 4.5: Allocation scenario for a (2,2) MIMO system.

the application of the VBLAST method we have a "candidate" allocation that maximizes the overall system capacity. User 1 is assigned to the 1st transmit antenna and user 2 is assigned to 2 nd transmit antenna. In order to accept this "candidate" allocation, the assumed data rates, as derived from VBLAST detection, have to be smaller than the capacity of the wireless links. Note that the order of detection depends on the SNR values. Specifically, signals with higher SNRs will be detected before signals with lower SNRs. The VBLAST detection order may be integrated into a matrix as:

$$
\text { detection_order }=\left[\begin{array}{cccc}
d t_{11} & d t_{12} & \cdots & d t_{1 N_{T}} \\
d t_{21} & d t_{22} & \cdots & d t_{2 N_{T}} \\
\vdots & \vdots & \ddots & \vdots \\
d t_{K 1} & d t_{K 2} & \cdots & d t_{K N_{T}}
\end{array}\right]_{K \times N_{T}}
$$

where $d t_{k n}$ denotes the user, that is detected by user $k$ in $n$th position after the VBLAST ordering. $N_{T}$ is the number of transmit antennas and $K$ the number of users. In our example the detection matrix is:

$$
\text { detection_order }=\left[\begin{array}{ll}
1 & 2 \\
1 & 2
\end{array}\right]
$$

According to that matrix, the 1st user has to be able to decode only the 1st signal (from Tx 1 ), because the signal for the 1st user is detected first. That means that the data rate of the 1st signal has to be smaller or equal to the capacity of the wireless link connecting antenna 1 of the BS to the 1st user: 


$$
R_{1} \leq C_{1}
$$

If we apply equation (4.2) to the capacity of the wireless link of the 1st user we have :

$$
C_{1}=\log _{2}\left(1+\gamma_{1,1}\right)
$$

The 2 nd user has to be able to decode the first antenna followed by the second antenna. The 2nd antenna signal is intended for the 2nd user, but the 2nd user has to be able to decode the 1st antenna's signal first in order to be able to decode the 2 nd signal. That means that the data rate of the 1st signal has to be smaller or equal to the capacity of the wireless link connecting the BS to the 2 nd user. The 1st signal received by the 2 nd user is the signal for the 1 st user, which means that the data rate of the 1st user has to be smaller or equal to the capacity of the wireless link of the 2 nd user. Thus, we have :

$$
R_{1} \leq C_{2}
$$

If we apply equation (4.2) to the capacity of the wireless link of the 2 nd user we have :

$$
C_{2}=\log _{2}\left(1+\gamma_{2,1}\right)
$$

In order for equations (4.15) and (4.17) to be satisfied simultaneously we must have

$$
R_{1} \leq \log _{2}\left(1+\min \left(\gamma_{2,1}, \gamma_{1,1}\right)\right)=C_{1}
$$

The feasible system capacity is $C_{o v}=C 1+C 2=>$

$$
C_{o v}=\log _{2}\left(1+\min \left(\gamma_{2,1}, \gamma_{1,1}\right)\right)+\log _{2}\left(1+\gamma_{2,2}\right)
$$

Note that the concept of feasible date rates is crucial to avoid error propagation in V-BLAST. 
Particularly, the feasibility constraint ensures error-free transmission and therefore no error propagation takes place in the SIC-receiver. In the case of data rates higher than the wireless link, the transmitted signal will be decoded incorrectly and all the subsequent detected signals will be affected leading to error propagation.

For a $\left(N_{T}, N_{R}\right)$ MIMO system with $K$ active users, given a specific allocation scenario ( $n$th mapping sequence) $\boldsymbol{P}_{n}(4.10)$, the detection vectors set $\boldsymbol{D}$ (the elements of the set are the vectors $D_{k, n}$ defined in equation (4.9)) and the post-detection SINR matrix $\boldsymbol{\Gamma}_{s i n r}$ (4.11), the flowchart of the algorithm that gives the feasible system capacity $C_{o v}$ may be expressed as in Figure 4.6. The pseudocode of the VBLAST feasibility test is given in appendix A (Figure A.3).

\subsubsection{Best user schemes}

The best user scheme is based on the idea that one user may be allocated to multiple transmit antennas unlike AA-RRS. Analytically, this scheme is based on the original AA-RRS scheme (section 4.3.2). According to the AA-RRS scheme, at each time slot, $N_{T}$ users are selected from $K$ users (in a round robin fashion), and the selected users form the scheduled user group (SUG). The proposed best user scheme, selects the users to form the SUG in a different way. Specifically, each transmit antenna $n$ is assigned to the user $k$ that realizes the best spatial channel. This means that one user may be allocated to multiple transmit antennas unlike AA-RRS. The criterion to choose the best spatial channel, is the post-detection SINR information. The post-detection SINR between transmit antenna $n$ and user $k$ is given by the equation (4.6).

The obvious drawback of the proposed scheme is that the selection criterion for the SUG may introduce some level of unfaimess between the users. Practically, the users with good spatial channels will be served more often than the users with poor spatial channels. In order to compensate for this unfaimess, the users to be selected are chosen in a round robin fashion. In the next sections, section 4.4.3.1 and section 4.4.3.2, we present the two implementations of the best user scheme.

\subsubsection{AA-RRS best user Scheme}

The proposed AA-RRS best user scheme, assigns each transmit antenna $n$ to the user $k$ that realizes the best spatial channel. The criterion to choose the best spatial channel, is the postdetection SINR information. The post-detection SINR between transmit antenna $n$ and user 


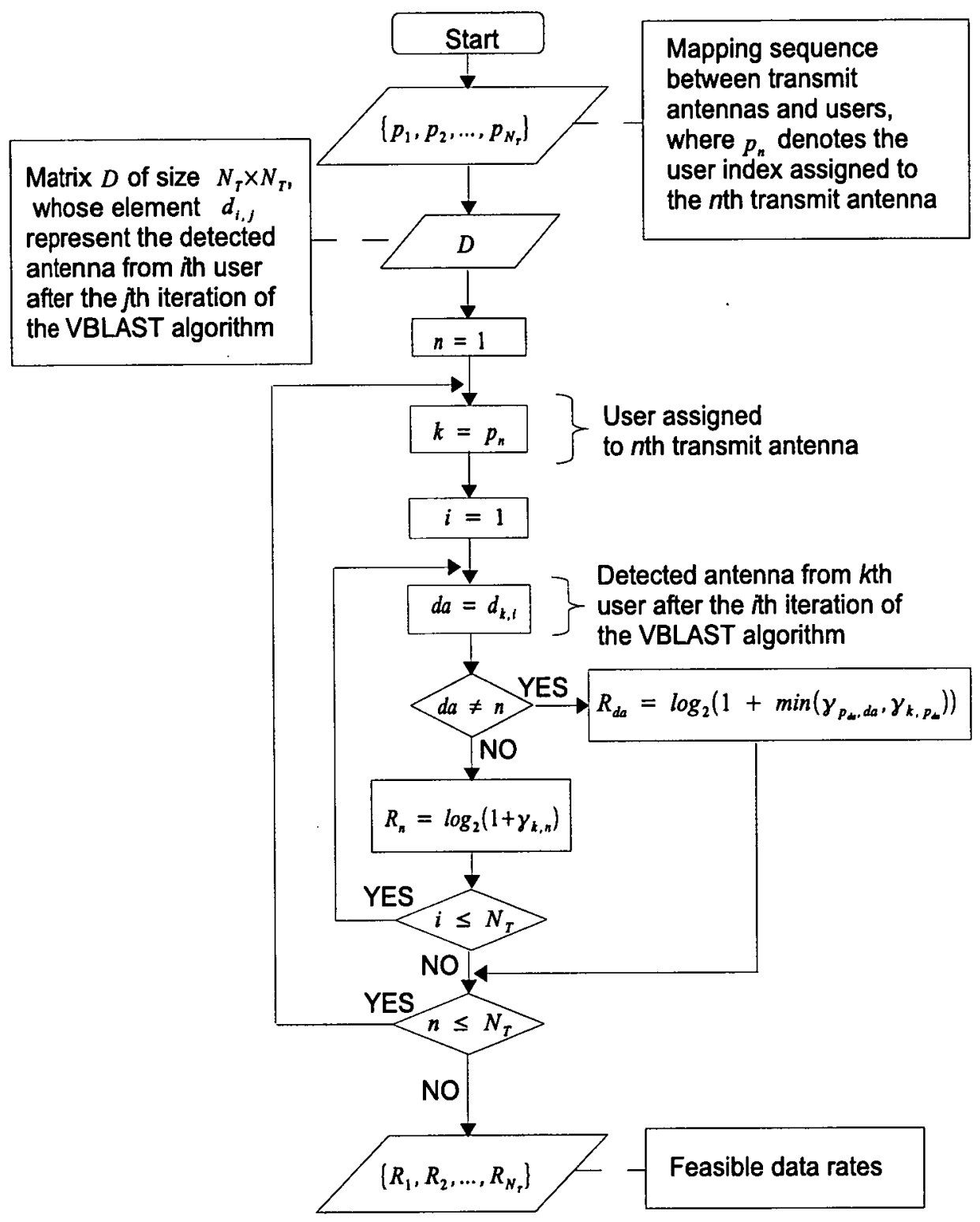

Figure 4.6: VBLAST feasibility test 
$k$ is given by the equation (4.6). The flowchart of the algorithm is drawn in Figure 4.7. The pseudocode is presented in appendix A (Figure A.4). The computational complexity of the AA-RRS best user scheme is the same as that of AA-RRS, $\mathrm{O}\left(N_{T}^{4}+N_{T} !\right)$.

\subsubsection{VBLAST AA-RRS best user Scheme}

The proposed VBLAST AA-RRS best user scheme, is similar to the AA-RRS best user scheme. The only difference is the employment of VBLAST detection instead of the MMSE detection that is used in AA-RRS algorithm. Because of that, the post-detection SINR between transmit antenna $n$ and user $k$ (that is used as criterion to form the SUG) is given by the equation (4.8). Note, that as with VBLAST AA-RRS, feasibility check is required.

The flowchart of this scheme is shown in Figure 4.8. The pseudocode is given in appendix A (Figure A.5). The computational complexity of the algorithm is the same as that of VBLAST AA-RRS, $\mathrm{O}\left(N_{T}^{5}+N_{T}\right.$ !).

\subsubsection{Antenna-Assisted (AA) MMSE fair Scheme}

The AA (Antenna-Assisted) MMSE fair scheme is based on the AA-RRS scheme. The main difference is the formation of the SUG. Specifically, the new AA MMSE fair does not form the SUG in a Round Robin fashion like the AA-RRS MMSE scheme does. The new scheme employs a new method to form the SUG. To describe the algorithm analytically, consider $K$ active users that are initially listed in such a way that each user is identified with a unique user index $k(k=1,2, \ldots, K)$. We separate the base station transmissions into equal groups of $\left\lceil K / N_{T}\right\rceil$ time slots (where $\lceil x\rceil$ rounds the number $\mathrm{x}$ to the nearest integer greater than or equal to $x$ ). During each of these time periods, all the $K$ users have to be served. In order to guarantee fair sharing of channel access among users, three rules have to be met:

1. All users have to be served during each time period of $\left\lceil K / N_{T}\right\rceil$ time slots,

2. no user can be allocated for a second time unless first all users have been served, and

3. each scheduled user is restricted to use one and only one transmit antenna per time slot.

During each group of $\left\lceil K / N_{T}\right\rceil$ time slots, we assume two user groups: a) SVUG (SerVed User Group), and b) UGSV (User Group to be SerVed). An SVUG consists of the users that have been already served, and it is an empty set at the beginning of the $\left\lceil K / N_{T}\right\rceil$ time slots. An UGSV group consists of the users still to be served. At each time slot, $N_{T}$ users are selected from the $\operatorname{UGSV}(t)$, and these users form a scheduled user group (SUG $(t))$ at the time slot. The 


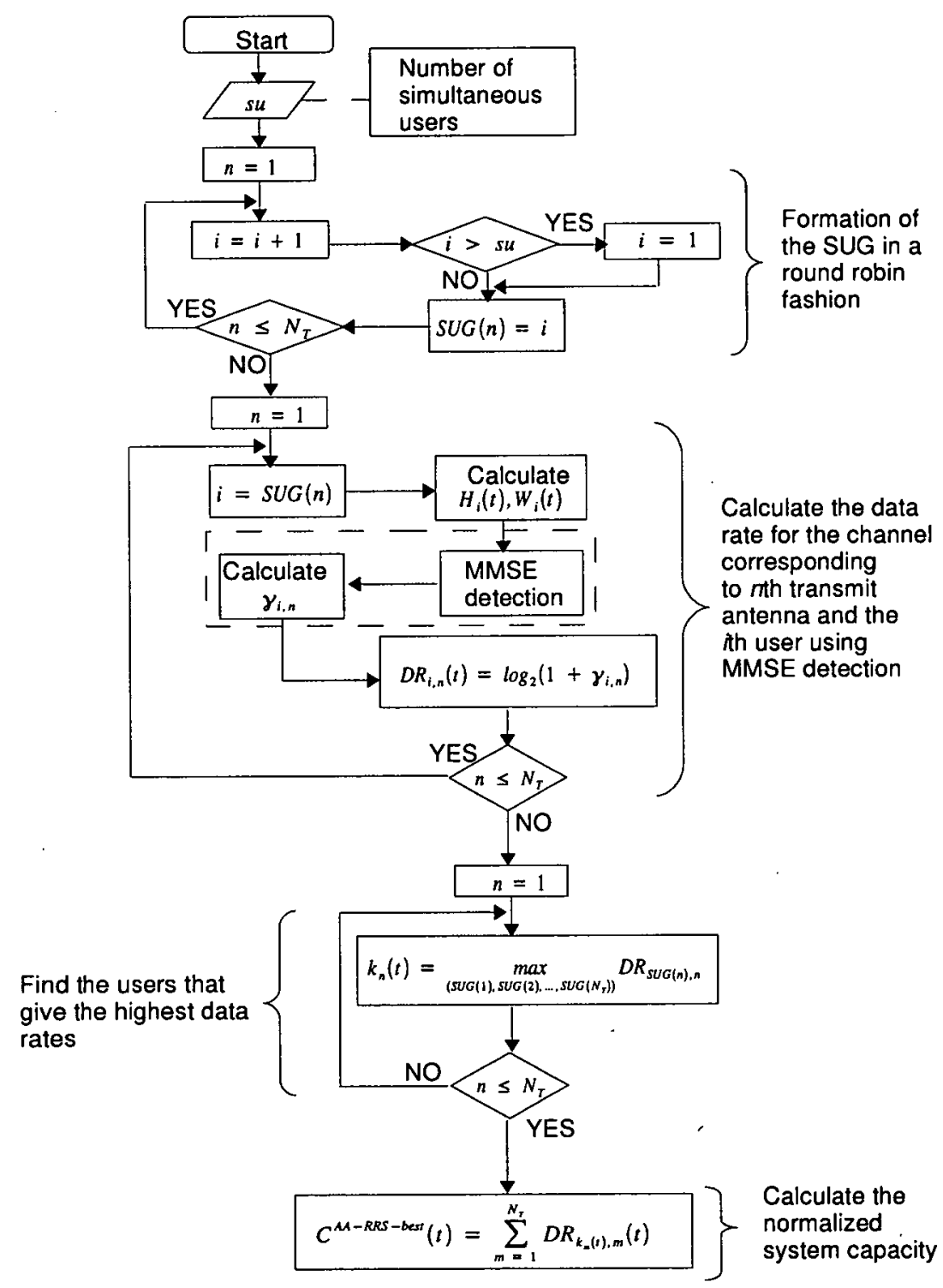

Figure 4.7: AA-RRS best user algorithm 


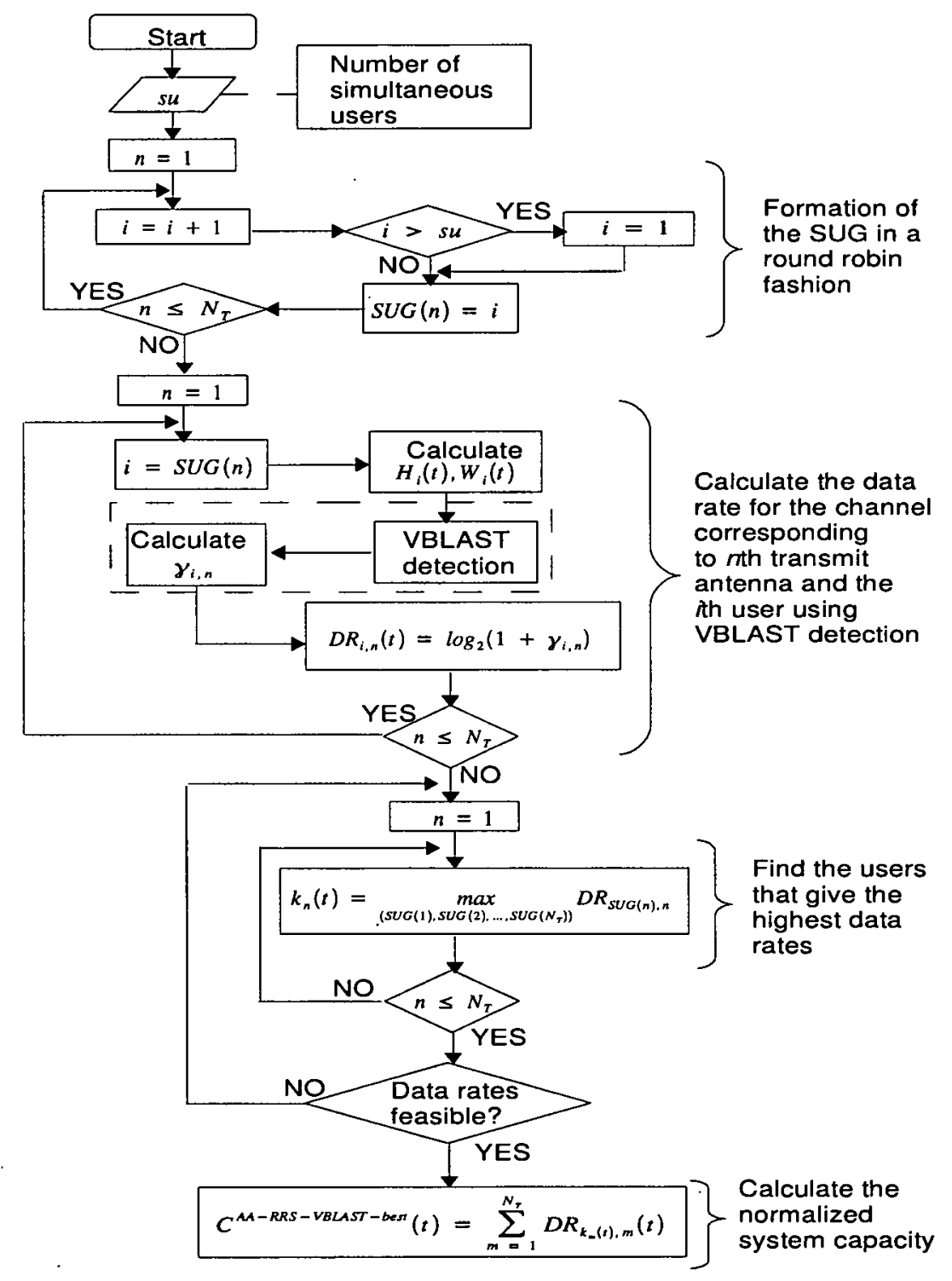

Figure 4.8: AA-RRS VBLAST best user algorithm 
selection of users for an SUG(t) is made according to the post-detection SINR information. Specifically, the $N_{T}$ highest values $\gamma_{k, n}(t)$ (equation (4.6)) determine the allocation scheme. The SVUG $(t)$ is updated with the users that are served at time slot $t$. And the UGSV for the next time slot $t+1$ is: $\operatorname{UGSV}(t+1)=\operatorname{UGSV}(t)-\operatorname{UGSV} t \cap \operatorname{SVUG}(t)$. If we attempt to calculate all the possible one-to-one mappings between the $N_{T}$ transmit antennas and the users belonging to the UGSV for $K>N_{T}$ at a time slot $t$ we have the following. Let us define $t_{a c t}$ as the time passed since the start of the last time period of $\left\lceil\frac{K}{N_{T}}\right\rceil$ time slots.

Then:

$$
t_{a c t}=t-\left\lceil\frac{K}{N_{T}}\right\rceil\left\lceil\frac{t}{\left\lceil\frac{K}{N_{T}}\right\rceil}\right\rceil
$$

with this definition, there are

$$
p\left(t_{a c t}\right)=\left(\left\lceil\frac{K}{N_{T}}\right\rceil-t_{a c t}-1\right) N_{T} !
$$

one-to-one mappings, and the average number of mappings per time slot is :

$$
p_{-} a v=\frac{\sum_{t_{a c t}=0}^{\left\lceil\frac{K}{N_{T}}\right\rceil-1}\left(\left\lceil\frac{K}{N_{T}}\right\rceil-t_{a c t}-1\right)}{\left\lceil\frac{K}{N_{T}}\right\rceil} N_{T} !=\frac{\left\lceil\frac{K}{N_{T}}\right\rceil-1}{2} N_{T} !
$$

In the special case of $K \leq N_{T}$, the algorithm is practically the same as the AA-RRS scheme. In AA-RRS scheme there are $N_{T}$ ! possible one to one mappings between $N_{T}$ scheduled users and $N_{T}$ transmit antennas. As we can see, the possible average mappings in the case of AA MMSE fair algorithm (for $K>N_{T}$ ) are $\frac{\left\lceil\frac{K}{N_{T}}\right\rceil-1}{2}$ times the mappings of the AA-RRS case. Taking into account that an appropriate choice of mapping may realize a strong diversity effect, we expect that AA MMSE will offer an advantage over AA-RRS scheme. On the other hand, more possible mappings means higher algorithm complexity.

As it already stated, we utilize the post detection SINR information (post-detection SINR matrix $\Gamma_{s i n r}$, equation (4.11)) for determining the users to form the SUG. As soon as the SUG is determined, we use again the post detection SINR, in order to find the most effective mapping, in the same manner as in the AA-RRS scheme. The system capacity, achieved by the best mapping (the mapping that maximizes the system capacity), can be expressed as : 
$C_{A A-M M S E-f a i r, E X S}(t)=\underset{\left\{\left(k_{1}, k_{2}, \ldots, k_{N_{T}}\right) \mid k_{i} \neq k_{j}, \text { if } i \neq j\right\}}{\arg \max } C_{A A-M M S E-f a i r}\left(t \mid\left(k_{1}, k_{2}, \ldots, k_{N_{T}}\right)\right)$

where

$$
\left.C_{A A-M M S E-f a i r}\left(t \mid\left(k_{1}, k_{2}, \ldots, k_{N_{T}}\right)\right)=\sum_{n=1}^{N_{T}} \log _{2}\left(1+\gamma_{k_{n}, n}\right)\right)
$$

and $\gamma_{k_{n}, n}$ is calculated from equation (4.6). Note that in equation (4.24), the demand $\left\{k_{i} \neq\right.$ $k_{j}$, if $i \neq j$ \} guarantees that user will not be allocated to multiple antennas.

The flowchart is drawn in Figure 4.9. Also, a pseudocode for this scheme is given in appendix A (Figure A.6).

The computational complexity of AA MMSE fair scheme is studied as a function of the number of active users $K$, and the number of transmit antennas $N_{T}$ (we assume that $N_{T}=N_{R}$ ). The algorithm first performs $K$ iterations of the MMSE algorithm to calculate $\gamma_{k_{n}, n}$ between the $N_{T}$ transmit antennas and $K$ users of the SUG. The MMSE algorithm has a complexity order of $\mathrm{O}\left(N_{T}^{3}\right)$. After the calculation of the post-detection SNR values, the algorithm requires $\left(K ! /\left(K-N_{T}\right)\right.$ ! comparisons to find the mapping sequence that maximizes the system capacity. Summarizing, the computational complexity of the AA MMSE fair scheme is $\mathrm{O}\left(K N_{T}^{3}+\frac{K !}{\left(K-N_{T}\right) !}\right)$

\subsubsection{Max-delete search (MDS) for the AA MMSE fair scheme}

The exhaustive search in equation (4.24) requires $N_{T}$ ! computations of equation (4.25), and this overhead may be excessively large for large $N_{T}$. To reduce computational requirements, we apply a simple heuristic mapping sequence (proposed in [137]), which is described as follows:

1) Select the largest post-detection SINR value among all the elements of the post-detection SINR matrix $\Gamma_{\text {sint }}(t)$ (4.11). Assume that $\gamma_{m_{1} n_{1}}$ is the largest one, then set $k_{n_{1}}$ to the user corresponding to the $m_{1}$ row of $\Gamma_{s i n r}(t)$.

2) Repeat the same operation $\left(N_{T}-1\right)$ more times for a modified $\boldsymbol{\Gamma}_{\text {sinr }}(t)$, where columns and rows associated with the selected post-detection SINR are deleted.

This mapping scheme is called max-delete search (MDS) [137]. If $\left(m_{1}, m_{2}, \cdots, m_{N_{T}}\right)$ is the resulting mapping sequence of the MDS, the system capacity may be expressed as 


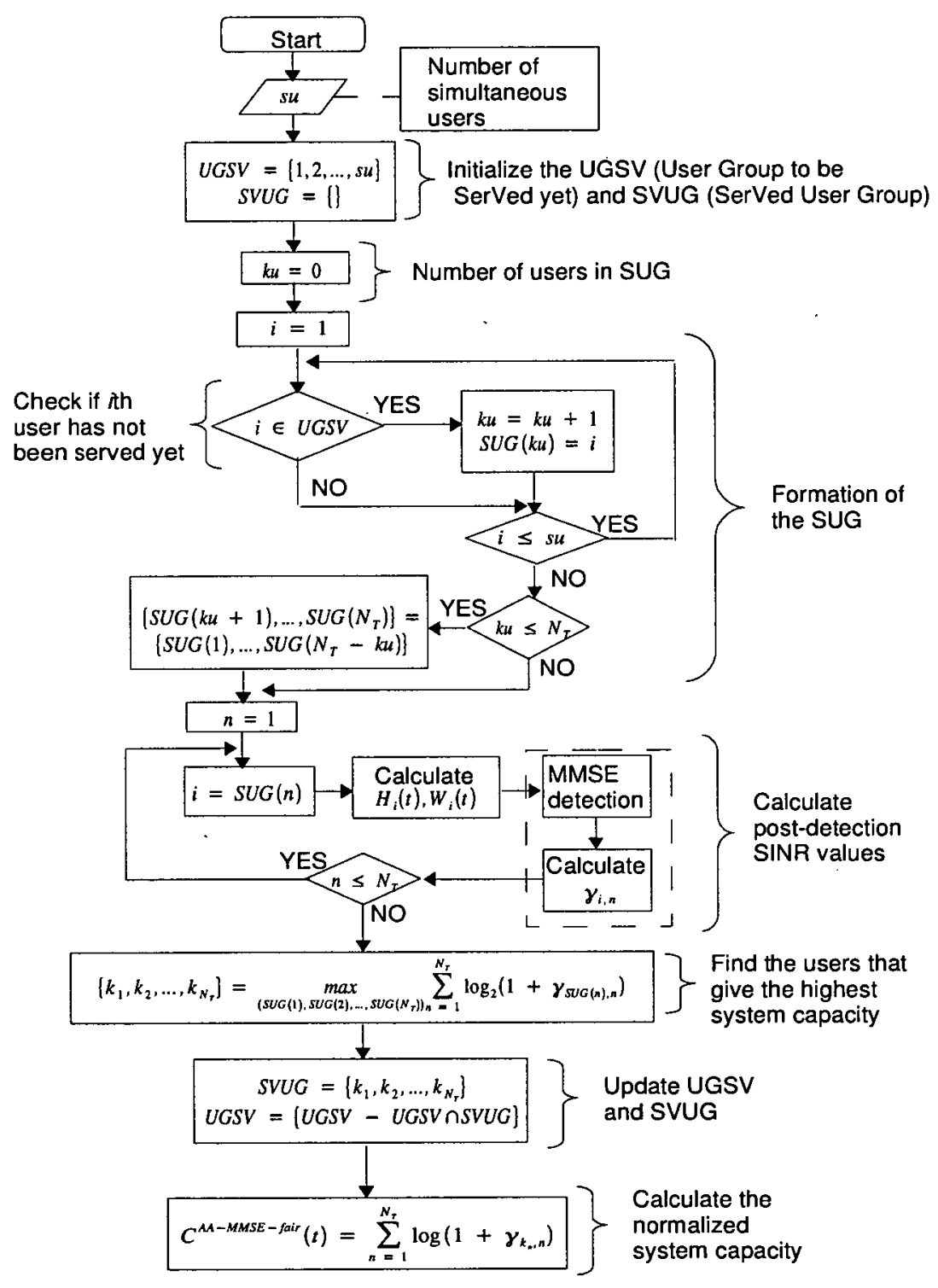

Figure 4.9: $A A-M M S E$ fair algorithm 


$$
C_{A A-M M S E-f a i r, M D S}(t)=\underset{\left\{\left(k_{1}, k_{2}, \ldots, k_{N_{T}}\right) \mid k_{i} \neq k_{j}, \text { if } i \neq j\right\}}{\arg \max } C_{A A-M M S E-f a i r}\left(t \mid\left(k_{1}, k_{2}, \ldots, k_{N_{T}}\right)\right)
$$

Note that the max-delete search selects the best channel at each stage, but unlike the best user scheme one user cannot be allocated to multiple transmit antennas.

\subsubsection{AA VBLAST fair Scheme}

This scheme is similar to the scheme described in section 4.4.4. The only difference is the use of VBLAST detectors at the receivers. The system capacity can be expressed as :

$C_{A A-V B L A S T-f a i r, E X S}(t)=\underset{\left\{\left(k_{1}, k_{2}, \ldots, k_{N_{T}}\right) \mid k_{i} \neq k_{j}, \text { if } i \neq j\right\}}{\arg \max } C_{A A-V B L A S T-f a i r}\left(t \mid\left(k_{1}, k_{2}, \ldots, k_{N_{T}}\right)\right)$

where $\left.C_{A A-V B L A S T-f a i r}\left(t \mid\left(k_{1}, k_{2}, \ldots, k_{N_{T}}\right)\right)=\sum_{n=1}^{N_{T}} \log _{2}\left(1+\gamma_{k_{n}, n}\right)\right)$ and $\gamma_{k_{n}, n}$ is calculated from equation (4.8). In the special case of $K \leq N_{T}$, the algorithm is the same as the VBLAST AA-RRS scheme. The flowchart of the algorithm is drawn in Figure 4.10. The pseusodocode is presented in appendix A (Figure A.7).

Similarly to the AA MMSE fair scheme, the computational complexity of the AA VBLAST fair scheme is $\mathrm{O}\left(K N_{T}^{4}+\frac{K !}{\left(K-N_{T}\right) !}\right)$, where $N_{T}^{4}$ represents the VBLAST detection.

\subsection{Simulation Model}

In this section, the system capacities for the RRS, AA-RRS and the proposed packet scheduling schemes (AA-RRS best user, VBLAST AA-RRS, VBLAST best user, AA MMSE fair and AA VBLAST fair) are evaluated and compared. The capacity for the RRS is calculated from equation (4.2) for 20,000 random realizations of the channel matrix in equation (4.1). To evaluate equation (4.2) for each channel matrix, the post-detection SINR values and the corresponding weight matrix of the receiver are computed using equations (4.6) and (4.4), respectively, under the assumption that MMSE detection is employed. Similarly, the capacity for the AA-RRS scheme is calculated from equation (4.5). The capacity for the VBLAST AA-RRS scheme is 


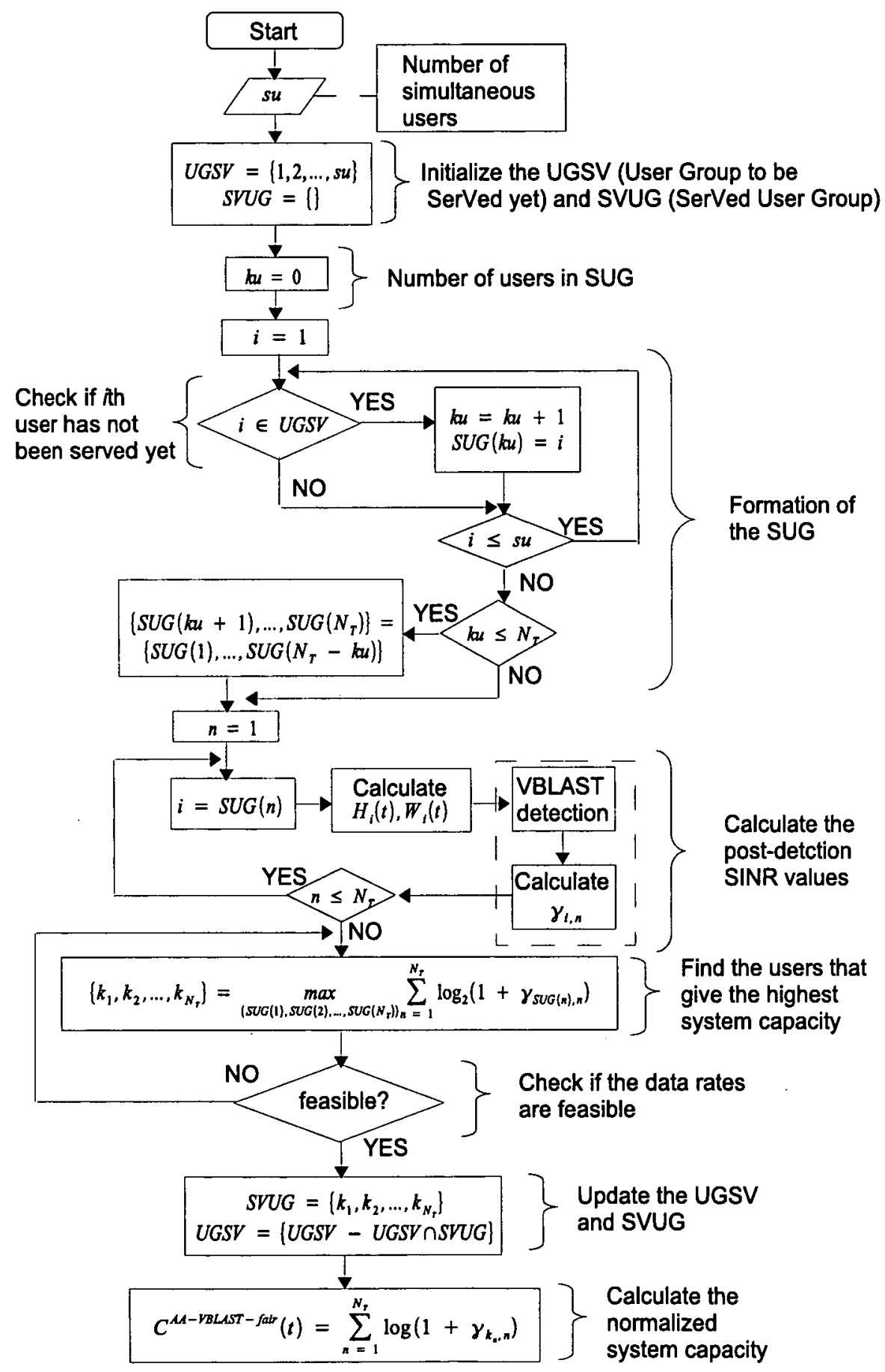

Figure 4.10: $A A-V B L A S T$ fair algorithm 
calculated from equation (4.12), where the weight matrix of the receiver and the corresponding SINR values are computed using (4.4) and (4.8), respectively, under the assumption that MMSE detection is employed. Finally, the capacities for the AA MMSE fair and AA VBLAST fair schemes are calculated from equations (4.24) and (4.27) respectively.

The expected capacity is defined as the capacity averaged over all possible channel realizations, and the $x \%$ outage capacity is defined such that the probability of the capacity at a time slot being less than the value is $x \%$. The path loss exponent $a_{D L}$ and log normal deviation of shadow fading $\sigma_{S}$ in equation (4.1) are assumed to be 3.7 and $8 \mathrm{~dB}$ respectively. We have used these specific values for path loss and shadowing, because these values give us a good match with the results provided by Oh-Soon Shin and Kwang Bok Lee [137]. In the simulation we assume different degrees of spatial fading correlation. The elements of $\boldsymbol{G}_{k}(t)$ are independent complex Gaussian random variables with zero mean and unit variance, in the case of uncorrelated channel. In the case of correlation, the elements of $\boldsymbol{G}_{k}(t)$ are determined using the model described in chapter 3 . Unless explicitly specified, the channel is assumed to be uncorrelated.

Delay time and data rate simulations are carried out in order to investigate the performance of different packet scheduling schemes. Signal-to-noise ratios at the cell boundary $\left(S N R_{0}\right.$ between $-10 \mathrm{~dB}$ and $10 \mathrm{~dB}$ were considered, were $\left(S N R_{0}\right)$ of $v \mathrm{~dB}$ corresponds to

$$
S N R_{0}=10^{\frac{v}{10}}
$$

Note that in the following simulation results we assume no error propagation at the receiver. This assumption is justified since the use of coding techniques such as convolutional or turbo codes are proved to be possible strategies to come close to the performance of a system where no error propagation occurs.

\subsubsection{Performance metrics}

In this section, we discuss the performance metrics that have been used in the simulation to characterize the performance of different packet scheduling schemes. In the following results, two basic performance concepts are studied: the system efficiency and the fairness. 


\subsubsection{System efficiency}

System efficiency is measured in terms of the system capacity (throughput). Any algorithm that improves the system capacity (throughput) is considered to be enhancing the system efficiency.

\subsubsection{2 fairness}

In a wireless network, fairness can be measured in terms of "effort" allocated to a flow by the network or in terms of "outcome" seen by the end-user [142]. "Effort" can be defined as the airtime devoted to a flow, whereas "outcome" is the throughput seen by the user. While in a wireline network, the two definitions can be used interchangeably, this is not the case in a wireless domain because of location and time-dependent variation in the perceived channel.

The round robin scheduler represents an ideal effort-fair algorithm because it allocates equal time to all the users in the system. However, as mentioned above, in a wireless domain, equal effort does not always yield equal outcome [143]. In our simulation the faimess is measured in terms of time delay. The time delay is the number of TTI's (Transmission Time Intervals). For

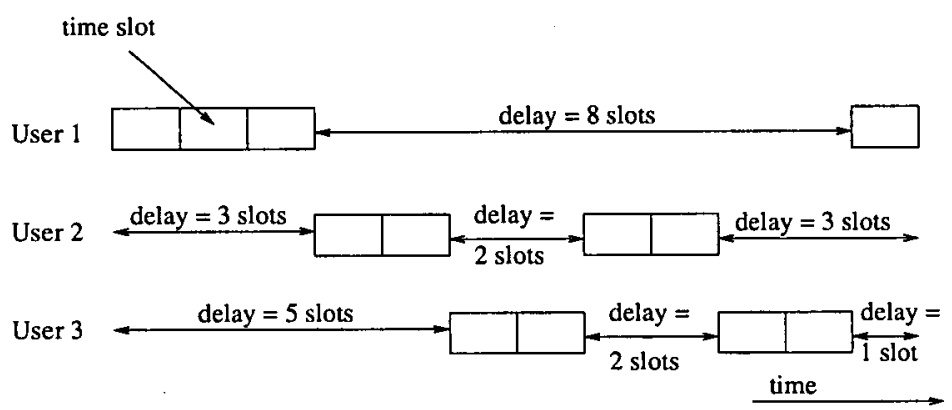

Figure 4.11: delay scenario for 3 users

each packet transmitted to a given user, the delay is defined as the number of idle time slots that have occurred since the last packet was sent to the same user. Figure 4.11 illustrates a delay scenario for 3 users. It can be seen that delay is measured in time slots as the time between two packet transmissions to the same user. Two different statistical parameters for the delay performance through the use of different scheduling schemes are studied. First, the average delay is a measure of the average waiting time $D$ for every user between each transmission. And second, the cumulative distribution function (CDF) of the delay variable $D$. This distribution is defined as

$$
F(d)=P(D \leq d)
$$


where $d$ is a specific value for the delay.

Another metric - the outage capacity - connected to delay and specifically to applications with delay constraint can be also considered as measure for the effort faimess [22]. Such application is the case, for example, in speech transmission over wireless channels. Similarly, the criterion for outcome fairness is chosen to be the throughput per scheduled user, which is the capacity allocated to each user of the SUG every time slot. Outage capacity is defined as the threshold $C t h$ which the system capacity will be below with a probability Pout.

\subsubsection{Results}

In this section, the performance - in terms of system capacities - of different scheduling schemes are evaluated and compared with each other. Also, results of different scheduling schemes are presented in terms of time delay and data rate per scheduled user. Wẹ separate the results into four subsections according to the following parameters.

1. Scheduling schemes (AA-RRS, AA-RRS best user, VBLAST AA-RRS, VBLAST AARRS best user, AA MMSE fair, AA VBLAST fair)

2. number of transmit and receive antennas

3. increasing $S N R_{0}$

4. spatial fading complex correlation

We will now discuss each category of results in turn.

\subsubsection{Scheduling Schemes}

In this section we measure the performance of various scheduling algorithms presented in this chapter. The platform for all the simulations in this section is a $(4,4)$ spatially uncorrelated MIMO system. We use the notation $\left(N_{T}, N_{R}\right)$ to denote a MIMO system with $N_{T}$ transmit antennas and $N_{R}$ receive antennas.

\section{AA-RRS Vs AA-RRS best user Vs VBLAST AA-RRS best user}

Figures 4.12(a) and 4.12(b) show the system capacities for a $(4,4)$ MIMO system. The transmit power is fixed to give $S N R_{0}=0 d B\left(S N R_{0}\right.$ denotes the median SNR power the cell bound- 


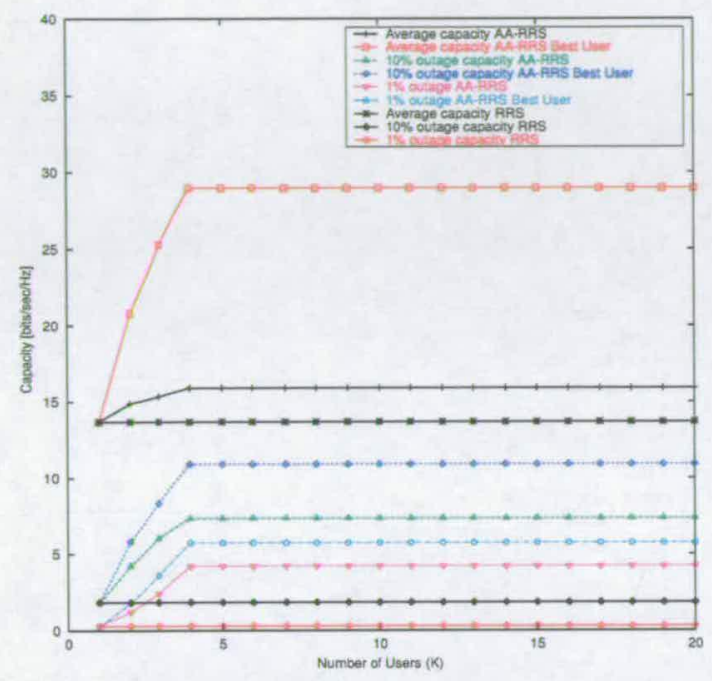

(a)

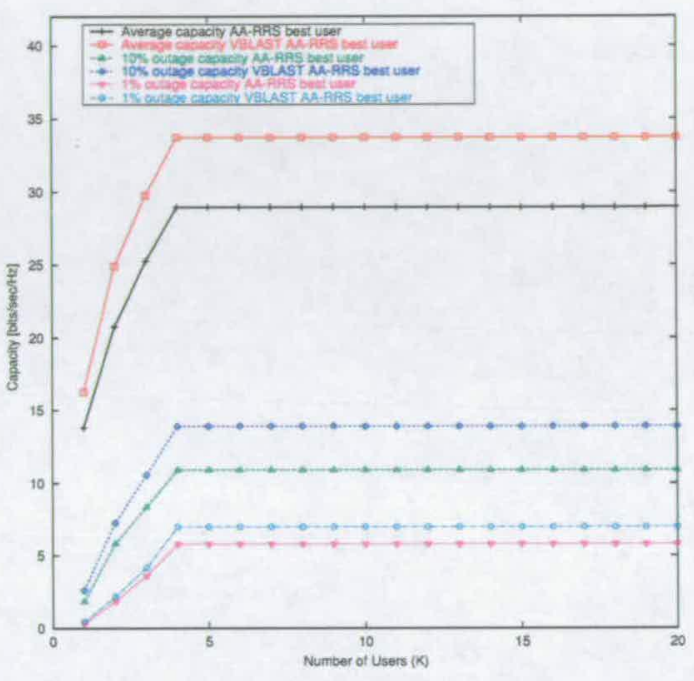

(b)

Figure 4.12: System capacities on a (4,4) MIMO system: (a)AA-RRS vs AA-RRS best user, (b) AA-RRS best user vS VBLAST AA-RRS best user

ary). Figure 4.12(a) shows that AA-RRS best user scheduling scheme provides a higher system capacity than the AA-RRS scheme and the RRS scheme. Specifically, for more than 4 users, there is a gain on average capacity of about $81 \%$ due to AA-RRS best user scheme over AARRS scheme and a gain of $116 \%$ over RRS scheme. In terms of outage capacities, the gain due to AA-RRS best user scheme is not as high as the gain in average capacities. Specifically, the gain on $10 \%$ outage capacity is only $51 \%$ over AA-RRS and the gain on $1 \%$ outage capacity is $31 \%$ over AA-RRS These results show that AA-RRS best user algorithm improves the system performance in terms of system capacities. Here, we must point out that in terms of outage capacities the AA-RRS best user scheme does not provide as much gain as for the average capacity, especially for a low outage probability. The reason for this is that the AA-RRS scheme selects a mapping between users in different conditions and transmit antennas, and this selection may reduce the low-tail probabilities of post-detection SINR rather than increase the average post-detection SINR. It should be noted that the outage capacity is a more important performance measure than the average capacity for applications with delay constraints [22]. Another point to mention is that the system capacity for both schemes increases with the number of users $k$, until $k$ approaches the number of transmit antennas $N_{T}$. This is because both schemes obtain a diversity effect from different users in the SUG. This diversity effect is bounded by the 
number of different users in the same SUG, but the maximum number of users in the SUG is equal to the transmit antennas $N_{T}$.

Figure 4.12(b) compares the AA-RRS best user scheme with VBLAST AA-RRS best user scheme in terms of system capacities. From the graph we see, that VBLAST AA-RRS best user scheme improves the system performance. Specifically, there is a gain $18 \%$ over AA-RRS Best User scheme in terms of average capacity. In terms of outage capacities, there is again of $27 \%$ on $10 \%$ outage capacity and gain of $20 \%$ on $1 \%$ outage capacity.

In Figure 4.13 we investigate the "outcome" fairness of the best user algorithms. This graph

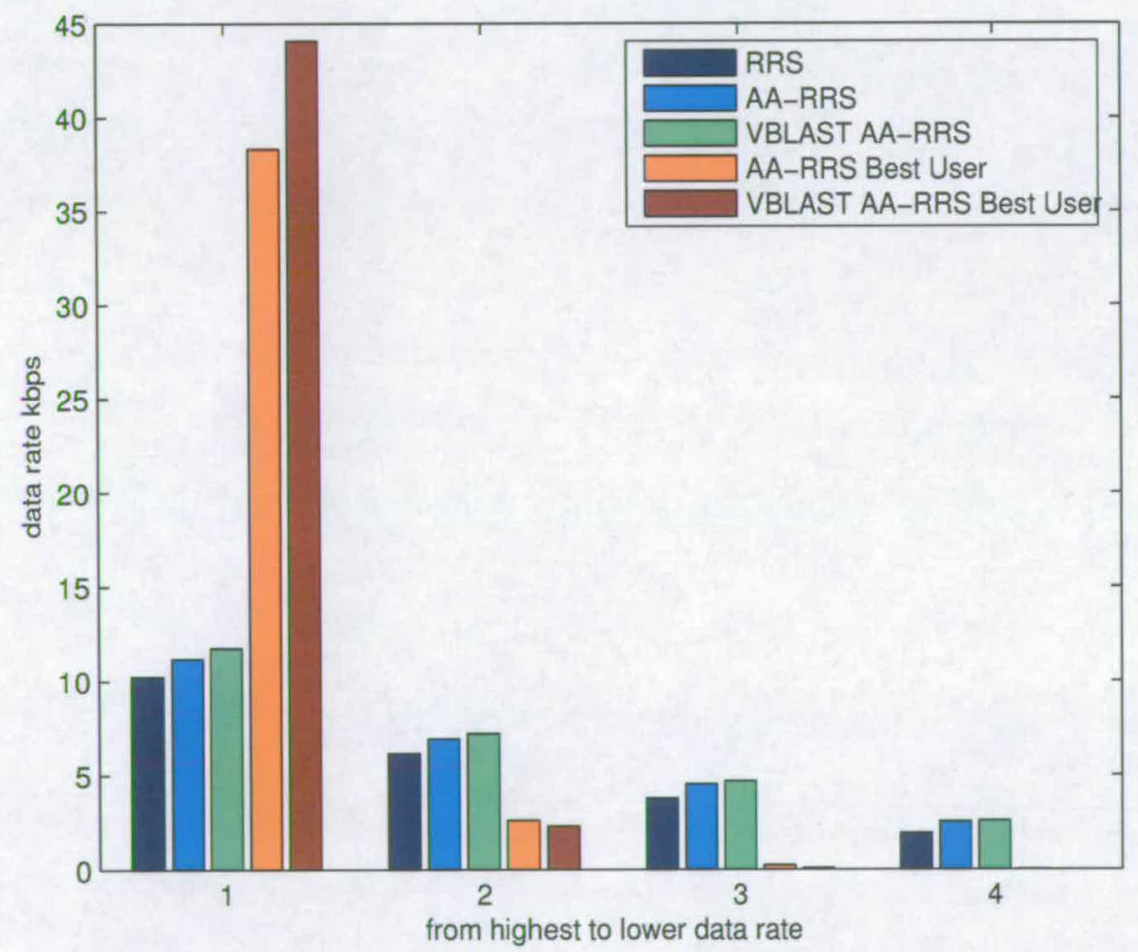

Figure 4.13: Average data rates per scheduled user every time slot for a $(4,4)$ MIMO system, SNR at the boundary equals $10 \mathrm{~dB}$

depicts the average data rates of each user in the SUG of a $(4,4)$ MIMO system. It is shown that the AA-RRS scheme provides a higher level of faimess among users, than the best user scheme. Namely, the AA-RRS scheduler shares the system capacity among all users of the SUG, which in the case of a $(4,4)$ MIMO system consists of 4 users. On the other hand, the 
best user scheduler in practice serves only 2 users at each time slot. The same results apply for the VBLAST AA-RRS best user scheduling. This chart shows that the RRS, AA-RRS and VBLAST AA-RRS algorithms are significantly more "outcome" fair than the AA-RRS best user and VBLAST AA-RRS best user schemes. It should be noted that the Best user AARRS scheme is slightly less unfair than the best user VBLAST AA-RRS scheme, which clearly favours the highest SINR user that is served each time slot.

Figure 4.14 shows the average throughput of 16 simultaneous users served by a $(4,4)$ MIMO

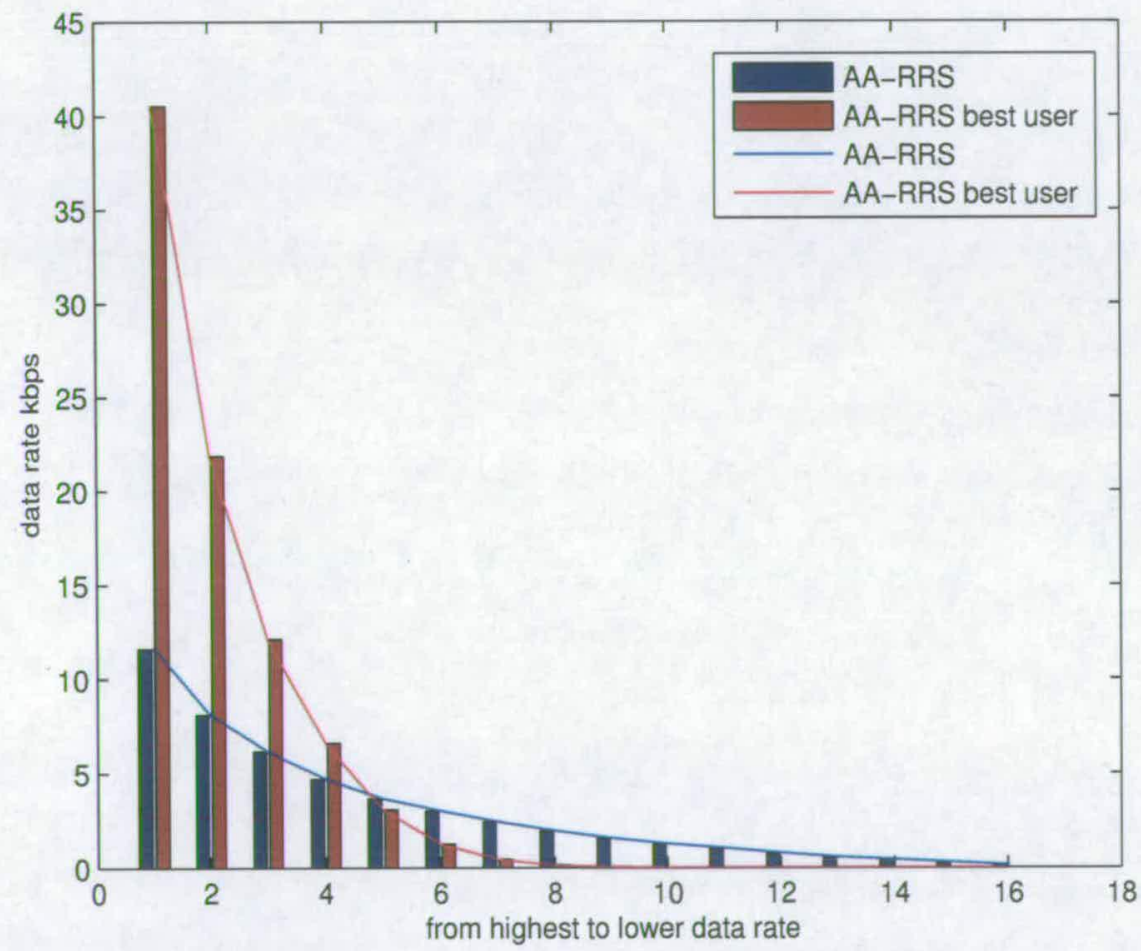

Figure 4.14: Average data rates per scheduled user every four time slots for a $(4,4)$ MIMO system that serves 16 simultaneous users, SNR at the boundary equals OdB

system during a period of 4 time slots. The transmit power is fixed to give $S N R_{0}=0 \mathrm{~dB}$. Two scheduling schemes are compared here the AA-RRS and the AA-RRS best user scheme. Four users are served every time slot, which means that 16 users are served after 4 time slots. From this graph it is obvious that the AA-RRS scheme provides much higher fairness among the simultaneous users than the best user implementation. Notice, that with the AA-RRS sched- 
uler all the 16 users share some portion of the available throughput. On the other hand with the AA-RRS best user scheduler only 6-7 users out of 16 are served at the same time period, although the overall system capacity of the AA-RRS best user scheme is much higher than the AA-RRS. In practice, the best user scheduler favors only some of the users that realize the best radio channels to the $\mathrm{BS}$.

\section{AA-RRS Vs VBLAST AA-RRS}

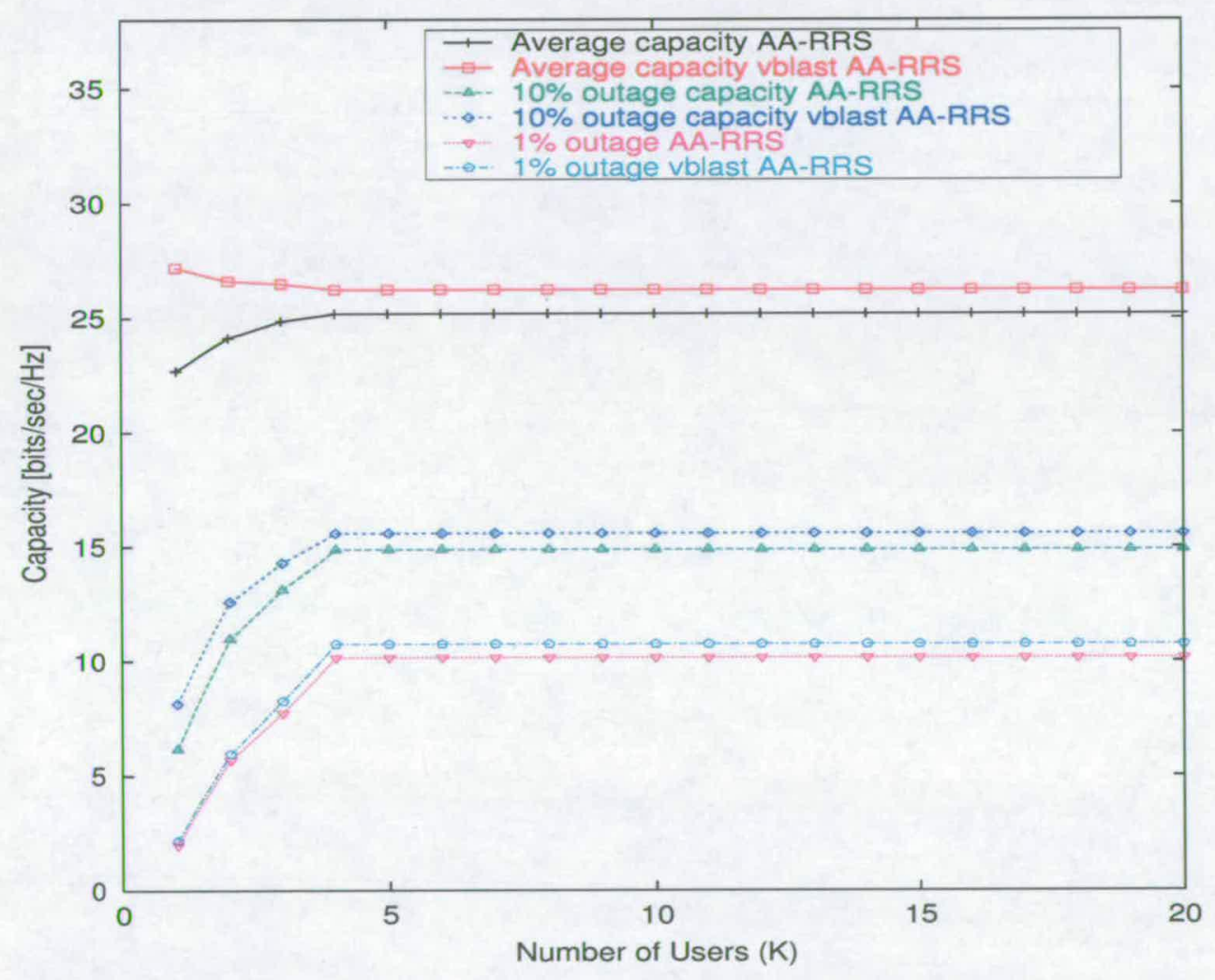

Figure 4.15: System capacities for AA-RRS MMSE and AA-RRS VBLAST scheduling schemes on a $(4,4)$ MIMO system.

The characteristics of the system capacity of a $(4,4)$ MIMO system with respect to the number of simultaneous users is shown in Figure 4.15. The transmit power is fixed to give $10 \mathrm{~dB}$ at the cell boundary $\left(S N R_{0}=10 d B\right)$. As we can see from this graph, the average system capacity of the proposed VBLAST AA-RRS scheduling scheme is increased about $4 \%$ over the conventional AA-RRS scheme. The results also indicate a similar improvement of the VBLAST AA-RRS scheme over the AA-RRS in terms of outage capacity. It should be noted 
that the average capacity of the VBLAST AA-RRS scheme does not increase with the number of users. That happens because the demand for feasible data rates cancels any possible capacity improvement that could occur due to multiuser diversity. Also another thing to point out here is that from the capacity point of view, the VBLAST RRS would be more efficient, but for delay sensitive systems we could apply the VBLAST AA-RRS scheme. This idea is supported by the findings of Figure 4.15. We notice that in the case of single user the VBLAST AA-RRS scheme performs better than any multiuser case, but the single user case of VBLAST AA-RRS scheme matches by definition the VBLAST RRS scheme. In the VBLAST RRS scheme the scheduler serves only one user per time slot. Going back to Figure 4.13, we compare the "outcome" fairness of the RRS, AA-RRS and VBLAST AA-RRS schemes. The RRS scheme, though it is supposed to be the most fair scheme, is slightly less fair than the AA-RRS and VBLAST AA-RRS schemes. Specifically, the 4th user in the RRS scheme is provided with 5.30 times lower data rate than the 1st user, but in the case of AA-RRS and VBLAST AA-RRS the 4th user is provided with 4.4 times less data rate than the first user, which means that the difference between the higher data rate user and the lower data rate is bigger in case of RRS than in the other two schemes.

III. AA-RRS VS AA MMSE fair and VBLAST AA-RRS VS AA VBLAST fair and AA MMSE fair Vs AA VBLAST fair

Figures 4.16(a) and (b) present the performance of the AA MMSE fair scheme and AA VBLAST fair scheme in terms of system capacities for a $(4,4)$ MIMO system. The transmit power is fixed to give $10 \mathrm{~dB}$ at the cell boundary $\left(S N R_{0}=10 \mathrm{~dB}\right)$. Figure 4.16 (a) compares the AA-RRS scheme with the AA MMSE fair scheme. It is obvious that there is a significant gain due to AA MMSE fair scheme for more than $N_{T}$ users (for $K \leq N_{T}$ the AA MMSE fair algorithm is the same as AA-RRS algorithm). Analytically, the system capacity for both schemes increases with the number of users $k$, until $k$ approaches the number of transmit antennas $N_{T}$. After that point, only the system capacity of the AA MMSE fair scheme keeps improving with respect to the simultaneous users. This is because the AA-RRS scheme obtain a diversity effect from different users in the SUG. This diversity effect is bounded by the number of different users in the same SUG, but the maximum number of users in the SUG is equal to the transmit antennas $N_{T}$. Also, the SUG in the case of AA-RRS algorithm is formed in a round robin fashion. On the other hand in AA MMSE fair algorithm, the SUG is not predefined by RRS. The SUG is formed by the selection of the users that have not been selected during the last time period, 


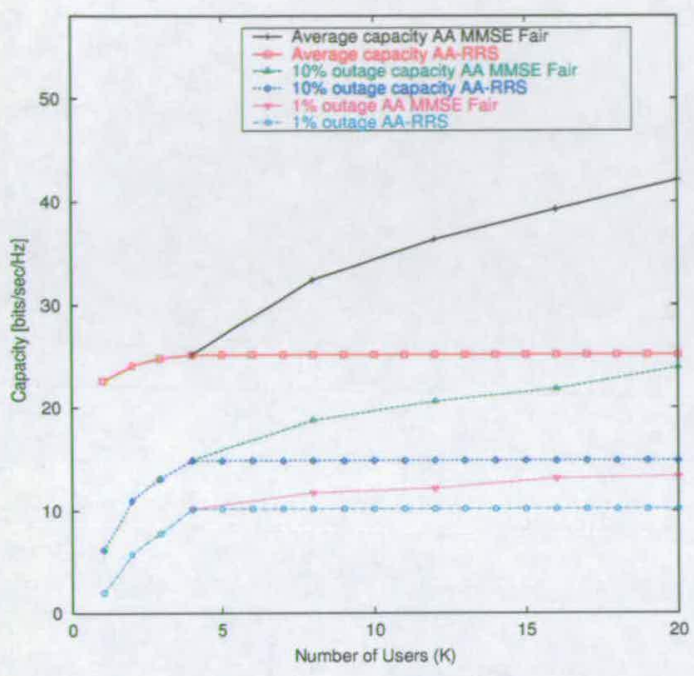

(a)

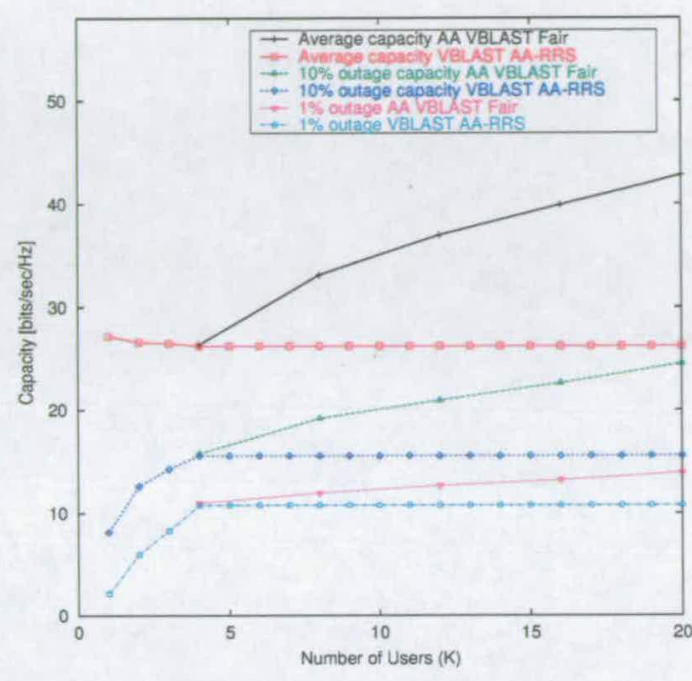

(b)

Figure 4.16: System capacities on a (4,4) MIMO system: (a) AA-RRS vS AA MMSE fair, (b) VBLAST $A A-R R S$ vS AA VBLAST fair

\begin{tabular}{|c|c|c|c|}
\hline Users & \multicolumn{3}{|c|}{ Delay in time slots } \\
\hline \hline & RRS & AA-RRS & AA-RRS fair \\
\hline \hline 4 & 3 & 0 & 0 \\
\hline 12 & 11 & 2 & 2 \\
\hline 16 & 15 & 3 & 3 \\
\hline 24 & 23 & 5 & 5 \\
\hline
\end{tabular}

Table 4.1: Time delay per scheduled user for a $(4,4)$ MIMO systems and for different scheduling schemes in a static environment.

and provide the highest post-detection SINRs. For a $(4,4)$ MIMO system with 20 simultaneous users, there is $69 \%$ gain on average capacity, $71 \%$ gain on $10 \%$ outage capacity and $50 \%$ gain on $1 \%$ outage capacity, due to AA MMSE fair.

In Figure 4.16(b) we compare the VBLAST AA-RRS scheme with the AA VBLAST fair scheme. Similarly to AA MMSE fair, the AA VBLAST fair improves significantly the system capacities for more than $N_{T}$ users (for $K \leq N_{T}$ AA VBLAST fair algorithm is the same as VBLAST AA-RRS algorithm).

Table 4.1 shows the the time delay per simultaneous user for a $(4,4)$ MIMO system in a static environment. By "static environment" we mean that the channel does not change every time slot. Specifically, in our simulation the channel changes every 100 time slots. This table (4.1) 
measures the "effort" fairness (airtime devoted to a flow). The AA-RRS fair provides the same delay times as the AA-RRS. At this point, note that both AA-RRS scheme and VBLAST AARRS scheme have exactly the same delay time distribution, because the decision on the users to be transmitted every time slot is made in round robin fashion. Because of the static nature of the channel, the delay times in case of AA-RRS fair scheme are the same as in the case of AA-RRS algorithm. Compared to plain RRS scheme, the AA-RRS and AA-RRS fair schemes outperform in terms of delay time, something very important for applications with delay constraints.

Figure 4.17 shows the the system capacities for a $(4,4)$ MIMO system in the case of AA

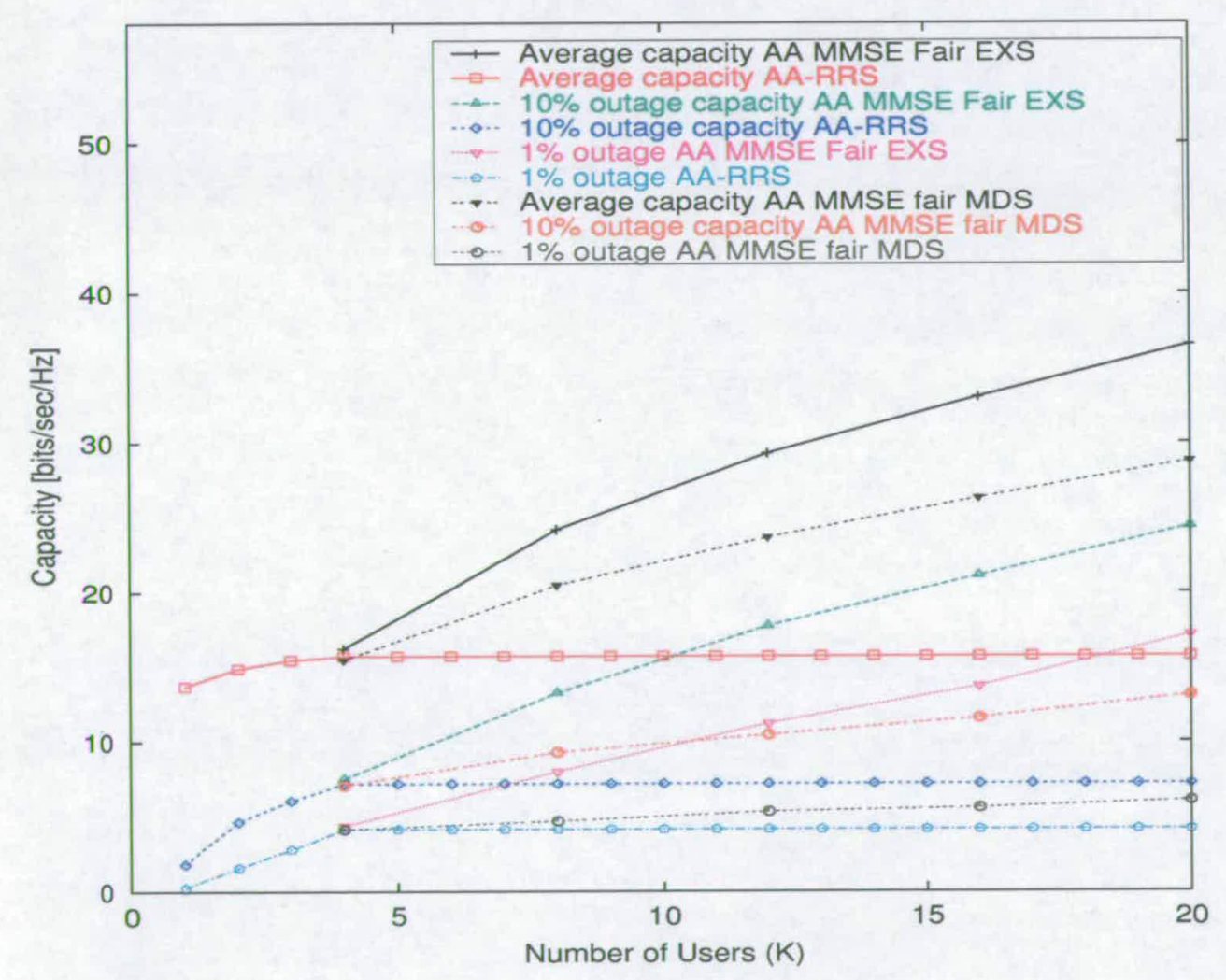

Figure 4.17: System capacities for AA MMSE fair and AA MMSE fair MDS (max-delete search) scheduling schemes on a $(4,4)$ MIMO system.

MMSE fair EXS (Exhaustive search) and AA MMSE fair MDS (max-delete search) scheduling schemes. The transmit power is fixed to give $S N R_{0}=0 \mathrm{~dB}$. The capacity difference between exhaustive search and max-delete search search is shown to be significant for systems 
where the number of the simultaneous users exceed the number of the transmit antennas.

In conclusion, the AA MMSE fair scheme and AA VBLAST fair scheme improve significantly the system performance in terms of system capacities and at the same time are more fair than the AA-RRS Best User scheme and VBLAST AA-RRS best user scheme.

\subsubsection{Number of Transmit and Receive Antennas}

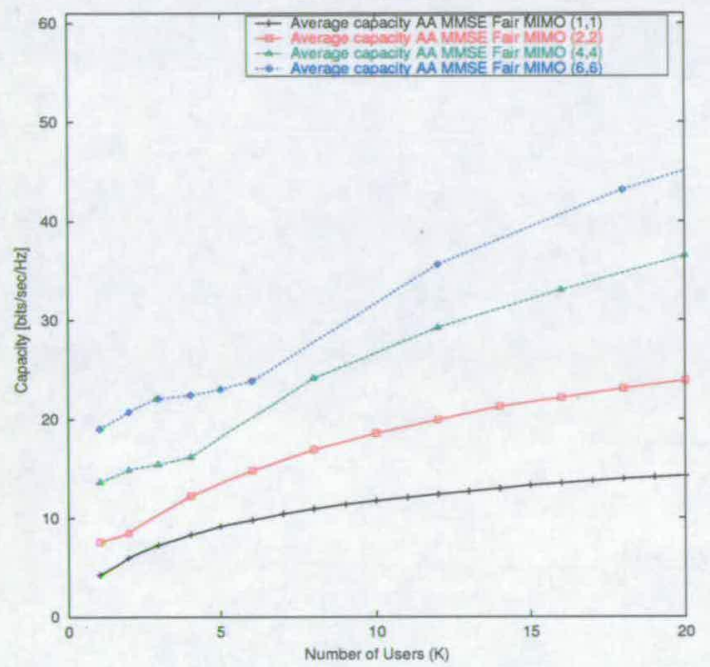

(a)

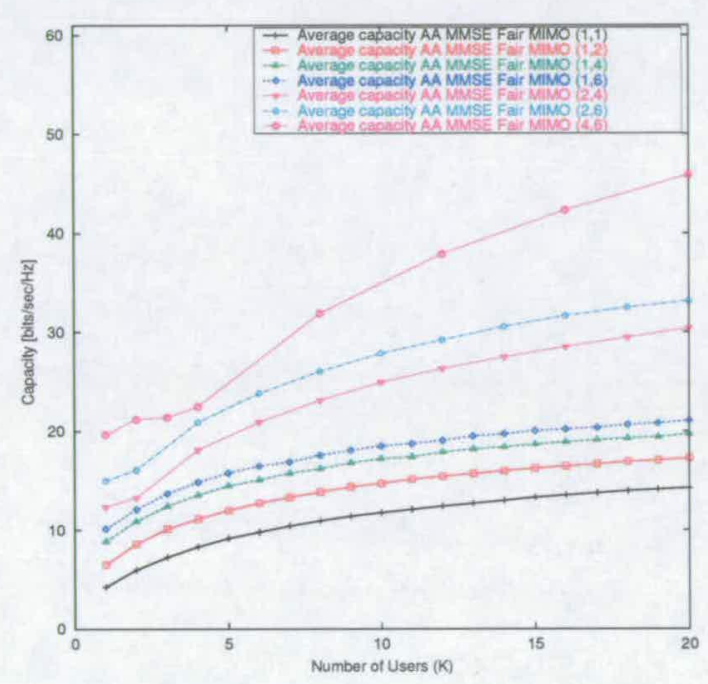

(b)

Figure 4.18: System capacities on a MIMO system: (a) symmetrical MIMO arrays, (b) asymmetrical MIMO arrays

In this section we investigate the antenna diversity effect of MIMO systems with different number of transmit and receive antennas. In the simulations carried out in this section the transmit power is fixed to give $0 \mathrm{~dB}$ SINR at the cell boundary $\left(S N R_{0}=0 \mathrm{~dB}\right)$. We separate our results into two groups. The first group is the symmetrical MIMO arrays, where the number of antenna arrays at the transmitter is equal to the number of the antenna arrays at the receiver. This group consists of the following MIMO systems: $(1,1),(2,2),(4,4)$ and $(6,6)$. The second group is the asymmetrical MIMO arrays, where there are more antenna arrays at the receiver than at the transmitter. This group consists of the MIMO systems: $(1,2),(1,4),(1,6),(2,4)$, $(2,6)$ and $(4,6)$. In Figure 4.18(a) and (b) we compare the system capacities values of the symmetrical MIMO arrays and asymmetrical MIMO arrays respectively, in case of AA MMSE fair scheduling, with respect to the number of simultaneous users. Note that for $K$ (number 
of simultaneous users) $\leq N_{T}$ the AA MMSE fair scheme is the same as the AA-RRS scheme, which means that the results of system capacities for $K \leq N_{T}$ are the same for these scheduling schemes (exactly the same stands in the case of AA VBLAST fair scheme and VBLAST AARRS scheme). In case of transmit diversity, namely when the number of transmit antennas is increasing we have the following results. First, we will analyze the results for $K \leq N_{T}$. Going from $(1,2)$ to $(2,2)$ results in a $18 \%$ increase in system average capacity. Going from $(1,4)$ to $(2,4)$ the increase is $36 \%$ and even going from $(1,4)$ to $(4,4)$ only produces an increase of $50 \%$. For $K>N_{T}$ we have, going from $(1,2)$ to $(2,2)$ results in a $38 \%$ increase, going from $(1,4)$ to $(2,4)$ the increase is $54 \%$ and from $(1,4)$ to $(4,4)$ the increase is $85 \%$. The above results point out that the AA MMSE fair algorithm is more efficient in exploiting the transmit diversity than the AA-RRS scheme (taking into account the results from Figure 4.16(a)). In case of receive diversity we have the following results for $K \leq N_{T}$. Going from $(2,2)$ to $(2,4)$ results in a $57 \%$ increase in system average capacity. Going from $(4,4)$ to $(4,6)$ the increase is $45 \%$ and going from $(2,2)$ to $(2,6)$ we notice an increase of $71 \%$. For $K>N_{T}$ we have, going from $(2,2)$ to $(2,4)$ an increase of $27 \%$. Going from $(4,4)$ to $(4,6)$ the increase is $24 \%$ and from $(2,2)$ to $(2,6)$ there is only a $39 \%$ increase. These results show that the AA MMSE fair is not as efficient with receive diversity as in the case of transmit diversity. In case of receive and transmit diversity we have the following results for $K \leq N_{T}$. Going from $(2,2)$ to $(4,4)$ results in a $77 \%$ increase in system average capacity. Going from $(2,2)$ to $(6,6)$ the increase is $150 \%$. For $K>N_{T}$, going from $(2,2)$ to $(4,4)$ we notice a $52 \%$ increase and going from $(2,2)$ to $(6,6)$ the increase is $100 \%$. In conclusion, the AA MMSE fair scheme exploits more efficiently than the AA-RRS scheme the available transmit diversity. On the other hand the gains are not so large in the case of receive diversity and in the case of combined receive and transmit diversity.

In Figure 4.19(a) and (b) we compare the system capacities values of the symmetrical MIMO arrays and asymmetrical MIMO arrays respectively, in case of AA VBLAST fair scheduling, with respect to the number of simultaneous users. As mentioned above, for $K$ (number of simultaneous users) $\leq N_{T}$ the AA VBLAST fair scheme is the same as the VBLAST AA-RRS scheme, which means that the results of system capacities for $K \leq N_{T}$ are the same for these scheduling schemes. Analyzing the simulation results to investigate the antenna arrays diversity effect, we notice that the graphs of system capacities for the AA VBLAST fair algorithm are similar to the ones studied before in the case of the AA MMSE fair scheme. This means, that the AA VBLAST fair algorithm exploits more efficiently than VBLAST AA-RRS scheme the transmit diversity, but is not so efficient in the case of receive diversity and in the case of 


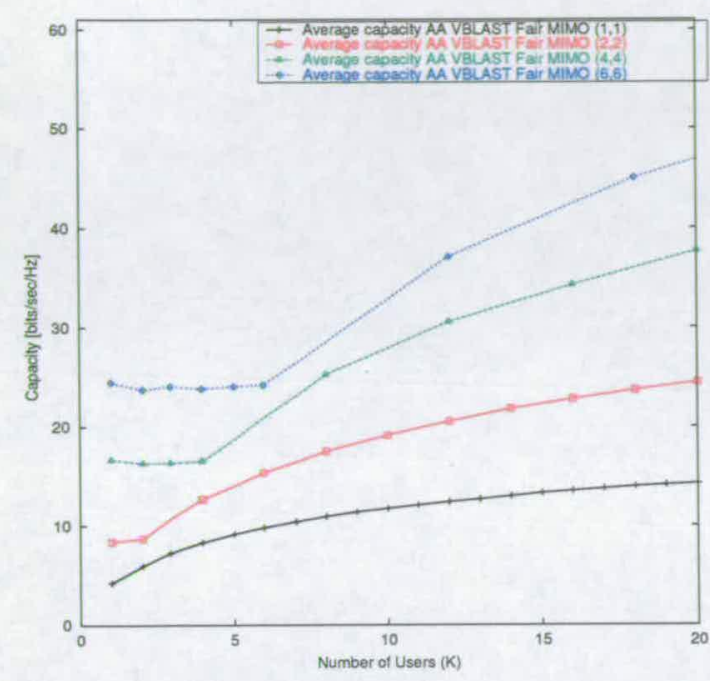

(a)

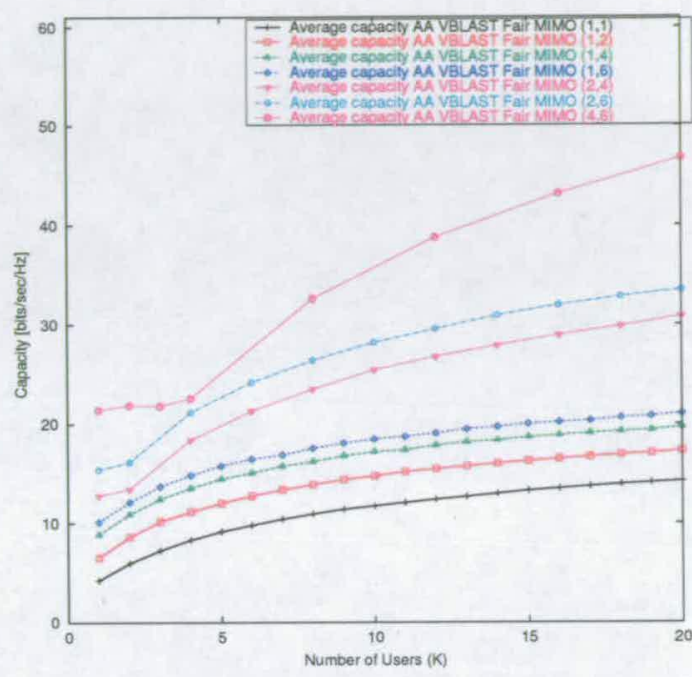

(b)

Figure 4.19: System capacities on a MIMO system: (a) symmetrical MIMO arrays, (b) asymmetrical MIMO arrays

combined receive and transmit diversity.

\subsubsection{Effect of the median SNR at the boundary $S N R_{0}$}

In this section we investigate the effect of the median SNR at the boundary $S N R_{0}$. Specifically for a given $S N R_{0}$ range, various packet scheduling schemes are presented and compared in terms of system capacities.

Figure 4.20 (a) shows the system capacities of a $(4,4)$ MIMO system with 20 simultaneous users. The AA-RRS best user scheme provides significantly higher system capacity than the AA-RRS scheme for any value of $S N R_{0}$ from $-10 \mathrm{~dB}$ to $16 \mathrm{~dB}$. Specifically, for $S N R_{0}=-10 \mathrm{~dB}$, there is a $110 \%$ gain on average capacity due to AA-RRS best user scheme over the AA-RRS scheme. Concerning the outage capacities we have gains of $68 \%$ and $44 \%$ on outage capacities of $10 \%$ and $1 \%$ respectively. For $S N R_{0}=16 \mathrm{~dB}$, there is a gain of only $54 \%$ on average capacity due to AA-RRS best user scheme over the AA-RRS scheme. Concerning the outage capacities we have gains of $38 \%$ and $28 \%$ for outage capacities of $10 \%$ and $1 \%$ respectively. We notice that for lower SNR values the AA-RRS best user scheme performs better with respect to the AA-RRS scheme. An explanation for this may be that in lower SNR, AA-RRS cannot exploit multiple antennas to achieve a diversity effect from multiple users because the condition of the 


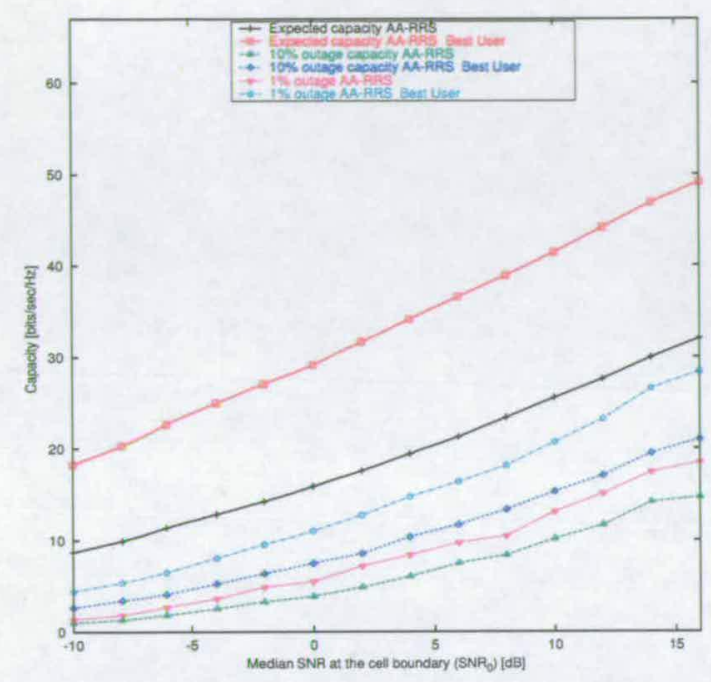

(a)

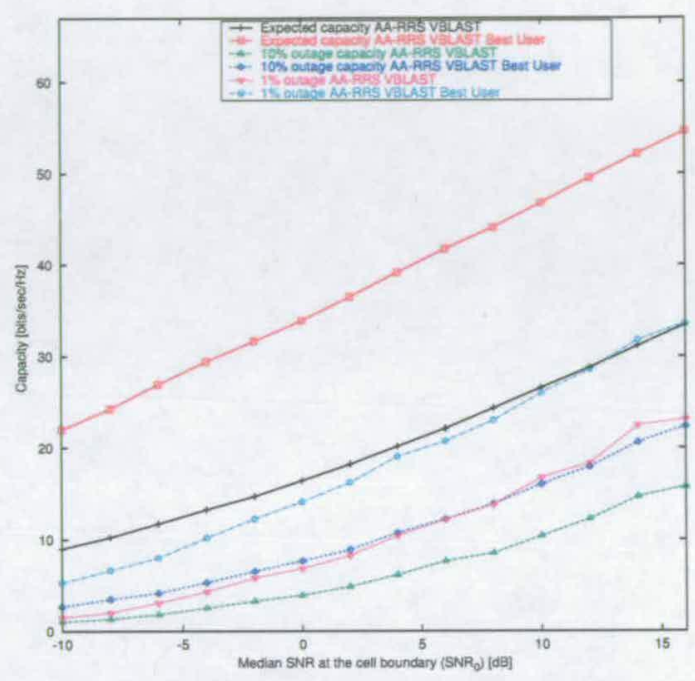

(b)

Figure 4.20: System capacities on a $(4,4)$ MIMO system for various values of SNR at the boundary: (a) AA-RRS vs AA-RRS best user, (b) AA-RRS VBLAST vs AA-RRS VBLAST best user

channel is not able to afford more than one user to be served each time slot. The AA-RRS best user scheme may be a good solution for bad channels.

Figure 4.20(b) shows the system capacities of a $(4,4)$ MIMO system with 20 simultaneous users. The VBLAST AA-RRS best user scheme provides significantly higher system capacity than the VBLAST AA-RRS scheme for any value of $S N R_{0}$ from $-10 \mathrm{~dB}$ to $16 \mathrm{~dB}$. Specifically, for $S N R_{0}=-10 \mathrm{~dB}$, there is a gain of $144 \%$ on average capacity due to VBLAST AA-RRS best user scheme over the VBLAST AA-RRS scheme. Concerning the outage capacities we have gains of $100 \%$ and $52 \%$ on outage capacities of $10 \%$ and $1 \%$ respectively. For $S N R_{0}=16 \mathrm{~dB}$, there is a gain of only $64 \%$ on average capacity. Concerning the outage capacities, we have gains of $53 \%$ and $48 \%$ on outage capacities of $10 \%$ and $1 \%$ respectively. As with AA-RRS best user, for lower SNR values the VBLAST AA-RRS best user scheme performs better with respect to the VBLAST AA-RRS scheme.

Figure 4.21(a) shows the system capacities of a $(4,4)$ MIMO system with 20 simultaneous users. The VBLAST AA-RRS scheme provides higher system capacity than the AA-RRS scheme for any value of $S N R_{0}$ from $-10 \mathrm{~dB}$ to $16 \mathrm{~dB}$. Figure 4.21(b) compares the AA MMSE fair scheme with the AA VBLAST fair scheme in terms of system capacities. The simulation has been carried out for a $(4,4)$ MIMO system with 16 simultaneous users. According to the 


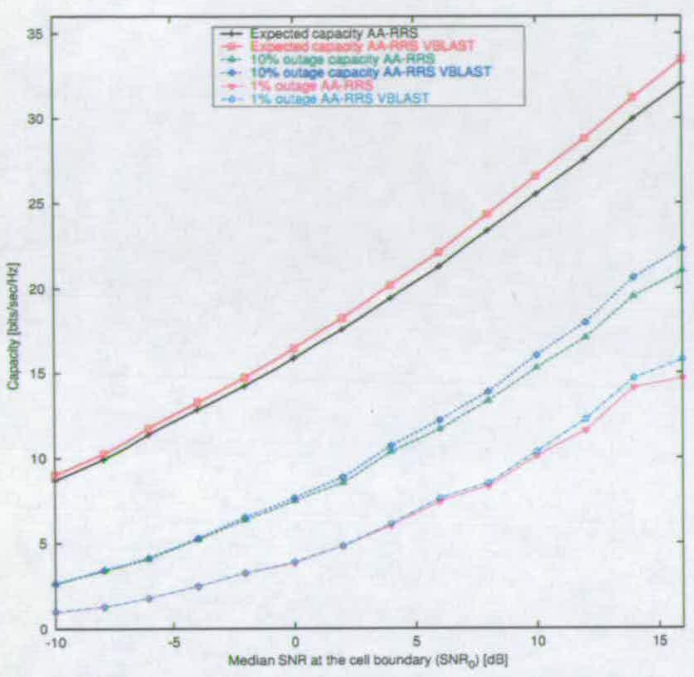

(a)

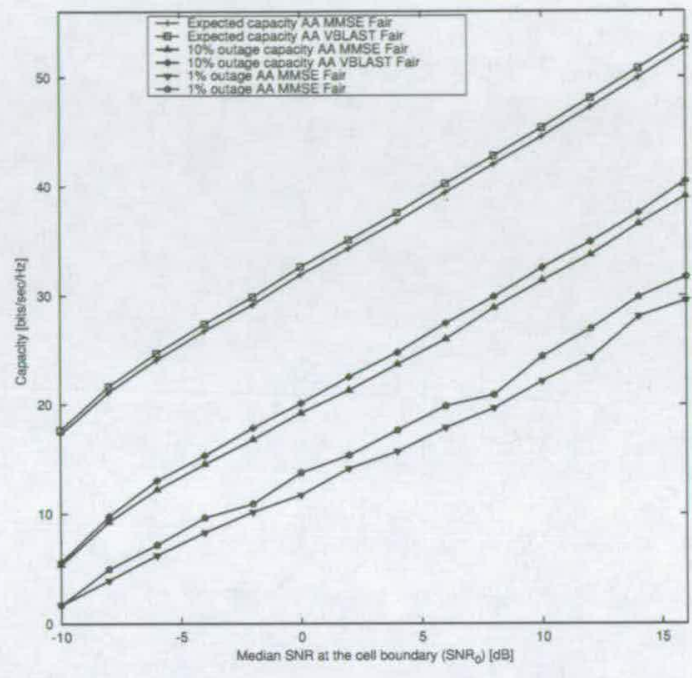

(b)

Figure 4.21: System capacities on a $(4,4)$ MIMO system for various values of SNR at the boundary: (a) AA-RRS vs VBLAST AA-RRS, (b) AA MMSE fair vs AA VBLAST fair

results, the AA VBLAST fair scheme outperforms the AA MMSE fair scheme in terms of system capacities for any value of $S N R_{0}$ from $-10 \mathrm{~dB}$ to $16 \mathrm{~dB}$. The results also indicate that improvement of the AA VBLAST scheme is more substantial in terms of the outage capacity, especially for a low outage possibility, than in terms of the average capacity. As we already mention in this chapter, the outage capacity is more important performance measure than the average capacity for applications with delay constraint.

\subsubsection{Spatial Fading Complex Correlation}

The effect of spatial fading complex correlation on different MIMO systems, is investigated. Many different correlation scenarios are investigated. The basic idea is to start from a decorrelated scenario, where both the Base Station (BS) and the Mobile Station (MS) are decorrelated (the mean absolute value of the coefficients is 0.1 ). Gradually, we increase the correlation either at the base station or at the mobile station or at both the base station and the mobile station. The correlation is increased from 0.1 to 0.9 , and the results for the system capacities are presented.

First, we look at the effect of correlation on a $(4,4)$ MIMO system for various packet scheduling schemes. In Figure 4.22 we measure the performance of the AA-RRS scheme and the VBLAST 


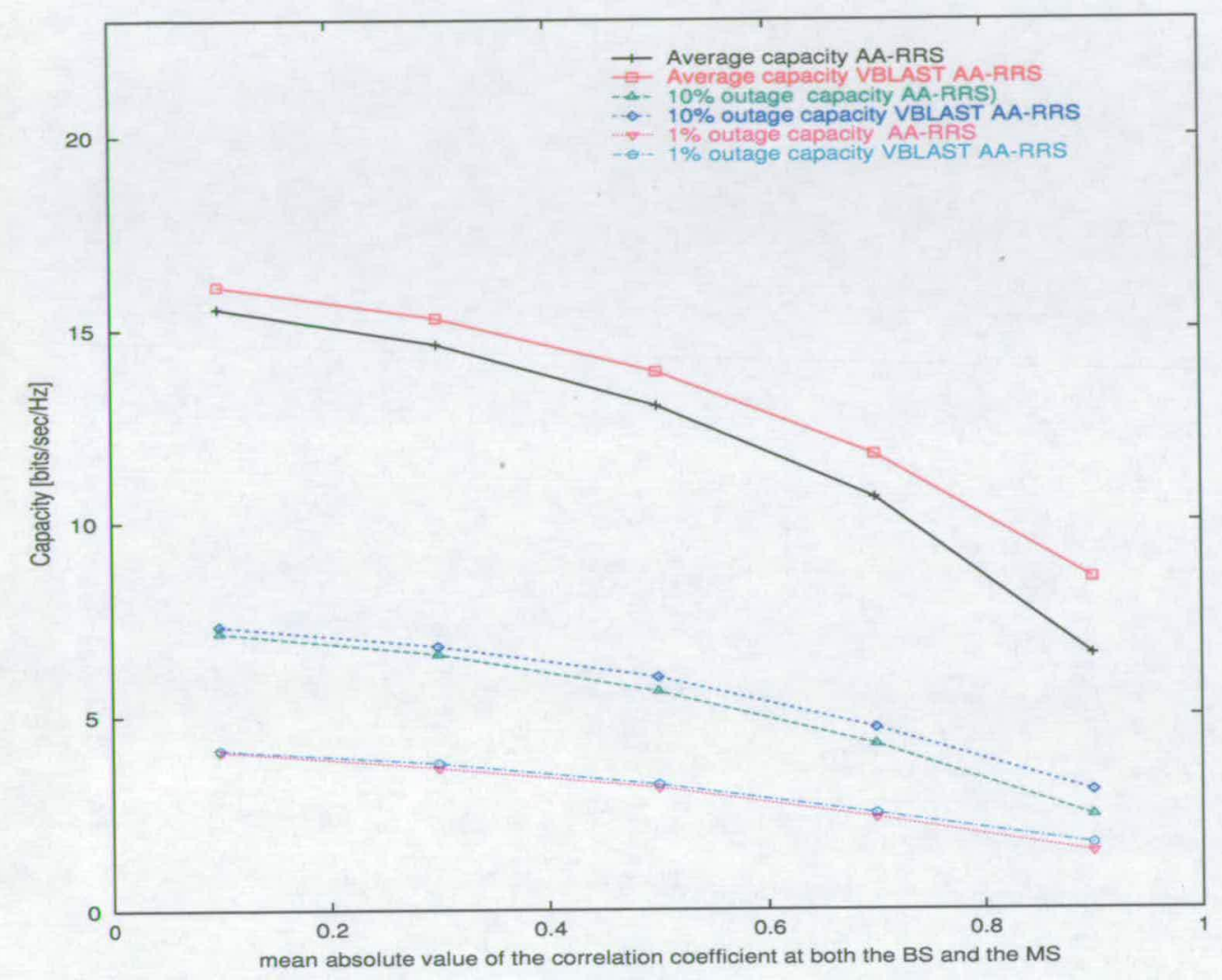

Figure 4.22: Effect of spatial correlation on a $(4,4)$ MIMO system, the correlation (at both the BS and MS) takes values from 0.1 to 0.9 .

AA-RRS scheme, in terms of system capacities. The transmit power is fixed to give $S N R_{0}=$ $0 d B$. The VBLAST AA-RRS algorithm provides a better performance than AA-RRS for any degree of correlation, and this improvements becomes higher as the levels of the correlation rise. Specifically, for high correlation, where the mean absolute value of the coefficients is 0.9 at both the BS and the MS (Figure 4.22), there is a $30 \%$ gain due to VBLAST AA-RRS on average capacity. Also, there are similar gains in outage capacities because of VBLAST AA-RRS. For outage capacity $10 \%$ the gain is $27 \%$ and for an outage capacity of $1 \%$ the gain is $15 \%$. If we compare these results with the results of the decorrelated scenario (correlation 0.1 at both ends) we will see that the VBLAST AA-RRS scheme is more efficient in case of correlated channels. Specifically, the gain due to VBLAST AA-RRS on average capacity is only $5.5 \%$, and the gains on outage capacities $10 \%$ and $1 \%$ are $2.4 \%$ and $1 \%$ respectively. The same conclusion can also be drawn from Figures 4.23(a) and (b). Figure 4.22(a) shows the 


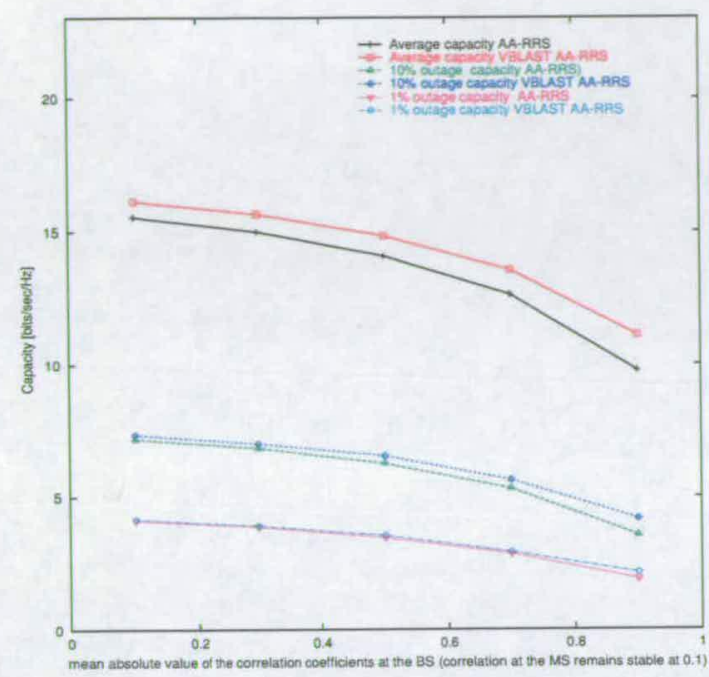

(a)

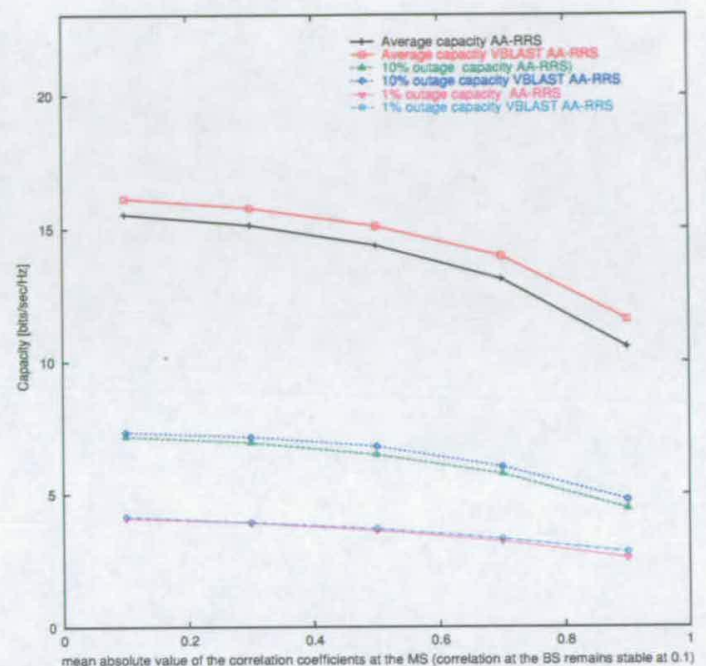

(b)

Figure 4.23: Effect of spatial correlation on a $(4,4) M I M O$ system: (a) the absolute value of the correlation coefficients at the BS takes values from 0.1 to 0.9 , and remains stable at MS (0.1), (b) the absolute value of the correlation coefficients at the MS takes values from 0.1 to 0.9 , and remains stable at $B S(0.1)$

system capacities, for different values of correlation at the BS in the case where the MS remains decorrelated ( 0.1 correlation). The gain on average capacity due to VBLAST AARRS, for the highest degree of correlation (0.9), reaches $13.5 \%$ and the gains for outage capacities $10 \%$ and $1 \%$ are $18 \%$ and $12.5 \%$ respectively. Figure 4.22 (b) shows the system capacities, for different values of correlation at the MS in the case where the BS remains decorrelated ( 0.1 correlation). From these results, we notice, first, that the VBLAST AA-RRS scheme is more efficient in case of higher correlation, and second, that when only the BS is correlated $(0.1)$ the gain of VBLAST AARRS over AARRS is higher than in the case when only the MS is correlated. Specifically, the gains in the case of correlated MS are, only $10 \%$ for average capacity and $9 \%$ for outage capacities. Figure 4.24 shows the system capacities on a $(4,4)$ MIMO system, where the transmit power is fixed to give $S N R_{0}=0 d B$. From the above graph, we notice that the increase of correlation does not affect the extent of the gain of the AA-RRS best user scheme over the AA-RRS scheme. To confirm that we compare the gain on system capacities due to AA-RRS best user scheme, in the case of decorrelated channel ( 0.1 correlation) with the case of highly correlated channel ( 0.9 correlation). This comparison shows no extra gain because of spatial correlation. The same results can be derived from Figures 4.25(a) and (b). Figure 


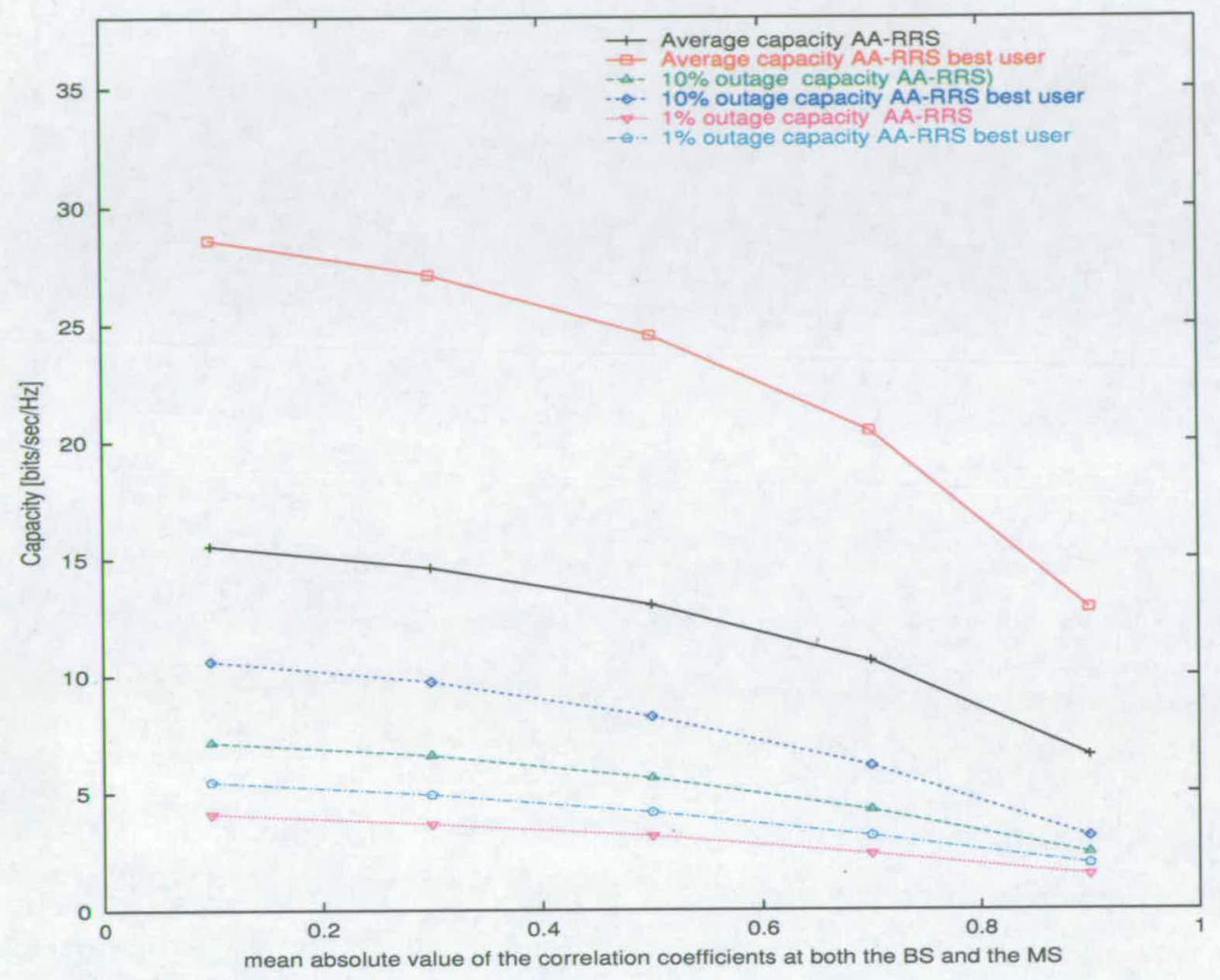

Figure 4.24: Effect of spatial correlation on a $(4,4)$ MIMO system, the correlation (at both the BS and MS) takes values from 0.1 to 0.9 .

4.25(a) shows the system capacities in case where the MS stays decorrelated and the BS takes different values of fading correlation from 0.1 to 0.9 . Figure 4.25 (b) covers the scenario where the BS remains decorrelated and the MS is correlated.

Figure 4.26 shows the system capacities on a $(4,4)$ MIMO system (serving 16 simultaneous users) under two different scheduling schemes, the AA MMSE fair scheme and the AA VBLAST fair scheme. The transmit power is fixed to give $S N R_{0}=0 d B$, where $\left(S N R_{0}\right.$ denotes the median SNR power the cell boundary. The AA VBLAST fair algorithm provides a better performance than AA MMSE fair scheme for any degree of correlation, and this improvements becomes higher as the levels of the correlation rise. Specifically, for high correlation, where the mean absolute value of the coefficients is 0.9 at both the BS and the MS, there is a $12 \%$ gain on average capacity due to AA VBLAST fair scheme. In the decorrelated case, this gain is restricted to only $2 \%$. Similar results also appear for the outage capacities. Specifically, 


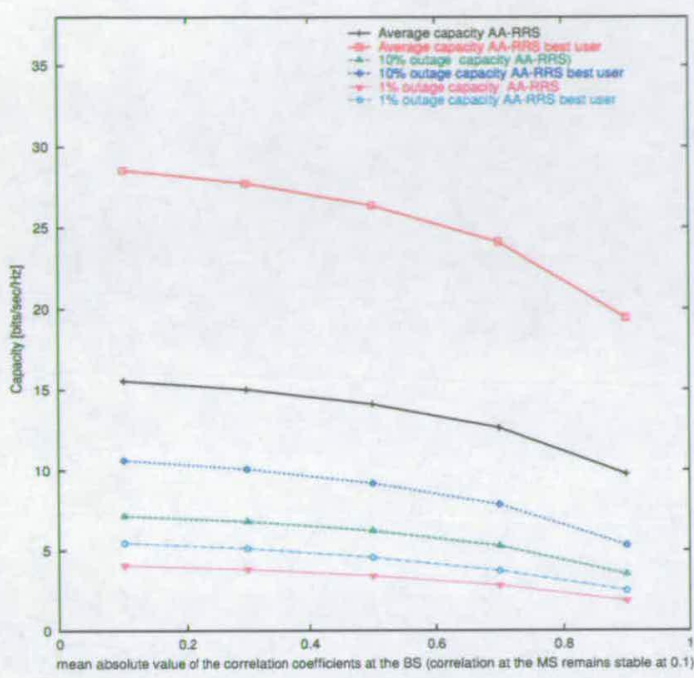

(a)

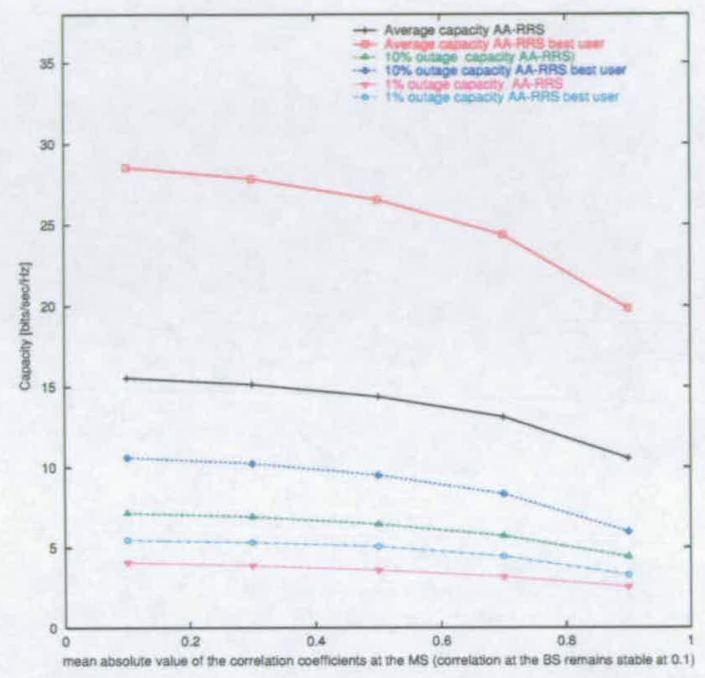

(b)

Figure 4.25: Effect of spatial correlation on a $(4,4)$ MIMO system: $(a)$ the absolute value of the correlation coefficients at the BS takes values from 0.1 to 0.9 , and remains stable at MS (0.1), (b) the absolute value of the correlation coefficients at the MS takes values from 0.1 to 0.9 , and remains stable at $B S(0.1)$

for outage $10 \%$ and $1 \%$, the gains due to AA VBLAST fair scheme in the case of correlated channel ( 0.9 correlation), are $18 \%$ and $24 \%$ respectively. On the other hand, in the case of decorrelated channel ( 0.1 correlation), gains on outage capacities are less than $3 \%$.

Figures 4.27 (a) and (b) confirm the above findings in the cases where only one of the BS or the MS remains decorrelated ( 0.1 correlation) and the other end of the channel suffers fading correlation from 0.1 to 0.9. For example in Figure 4.27(a) for the case of 16 simultaneous users we have the following results. There is a $7 \%$ gain on average capacity due to AA VBLAST fair over AA MMSE fair for highly correlated channel ( 0.9 correlation). The gains due to AA VBLAST fair on outage capacities $10 \%$ and $1 \%$ are $12 \%$ and $18 \%$ respectively. For the "decorrelated" case the gains due to AA VBLAST on average capacity and outage capacities $10 \%$ and $1 \%$ are less than $3 \%$. These results point out an extra gain due to VBLAST detection when the spatial correlation is increasing.

Figure 4.28(a) and (b), and 4.29(a) and (b) show the effect of spatial fading correlation on system capacities of MIMO systems of different number of transmit and receive antennas. Two different packet scheduling schemes are simulated and compared, the AA-RRS scheme and VBLAST scheme. The transmit power is fixed to give $S N R_{0}=0 d B$. Figure 4.28 (a) refers 


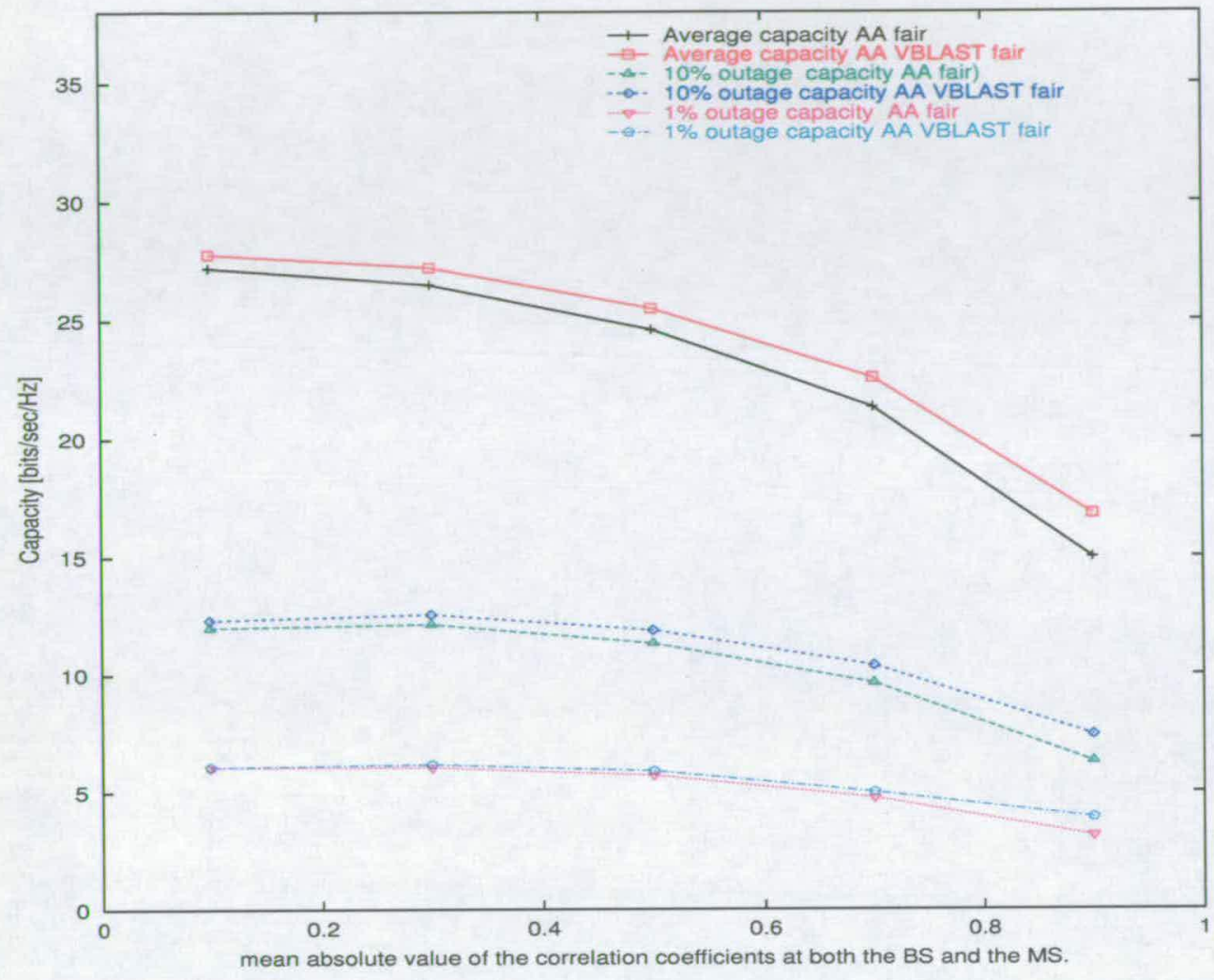

Figure 4.26: Effect of spatial correlation on a (4,4) MIMO system - 16 users, the correlation (at both the $B S$ and $M S$ ) takes values from 0.1 to 0.9 .

to a $(2,2)$ MIMO system. Going from decorrelated channel to highly correlated channel (or from 0.1 to 0.9 correlation), the gain on average capacity, due to VBLAST AA-RRS scheme, is increasing from $3.8 \%$ to $34 \%$. For a $(6,6)$ MIMO (Figure 4.28 (b)) the respective gain is increasing from $3 \%$ to $25 \%$. From the above results we can see that increasing the number of antennas at the receiver and the transmitter does not improve the efficiency VBLAST AA-RRS scheme. The reason for that is that increasing the number of antennas is slightly decreasing the capacity gain for the VBLAST AA-RRS scheme, because though the diversity effect is supported, more antennas implies more users at the SUG which results in more constraints on the feasibility test (section 4.4.2). The same conclusion can be drawn from Figures 4.29(a) and (b), where the effect of spatial correlation on the AA-RRS and VBLAST AA-RRS schemes is investigated for a $(2,4)$ MIMO system and a $(4,6)$ MIMO system. Conclusively, as larger the antenna arrays of the MIMO system as smaller the gain due to employment of VBLAST 
Exploiting Multiuser Diversity for MIMO Cellular Systems using RRS Packet Scheduling and the VBLAST Receiver

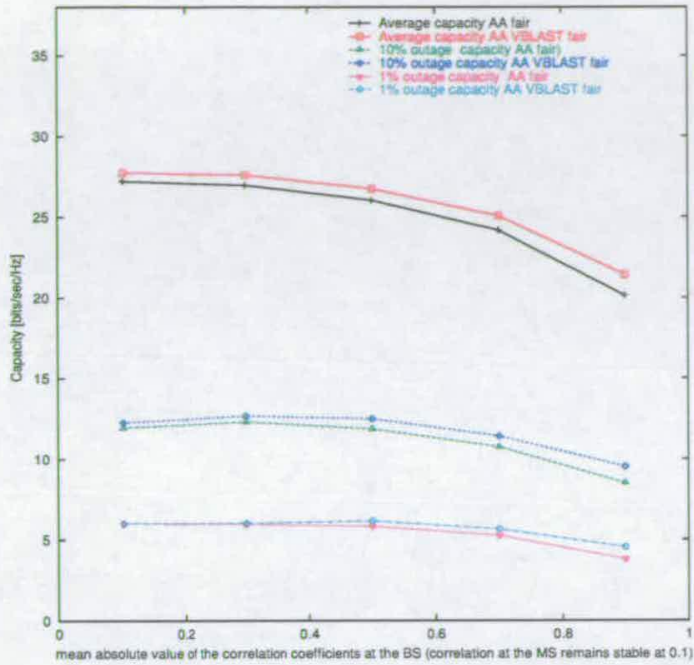

(a)

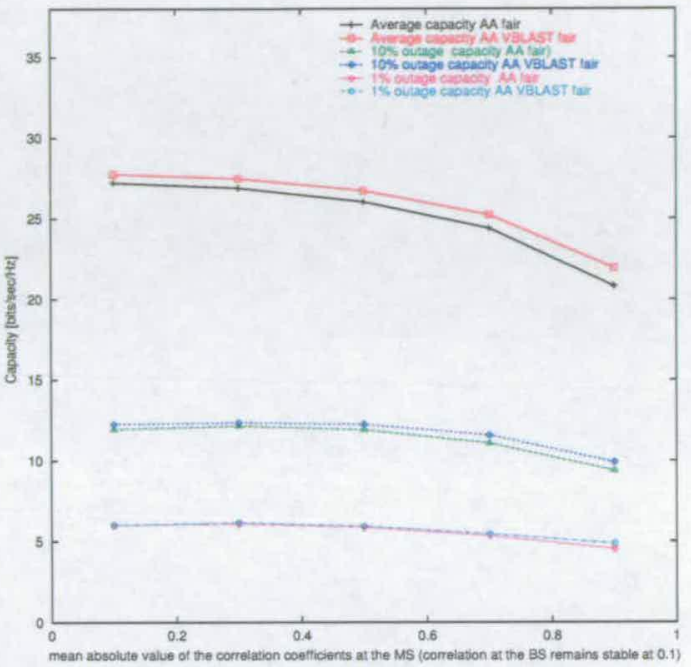

(b)

Figure 4.27: Effect of spatial correlation on a (4,4) MIMO system - 16 users: (a) the absolute value of the correlation coefficients at the BS takes values from 0.1 to 0.9 , and remains stable at MS (0.1), (b) the absolute value of the correlation coefficients at the MS takes values from 0.1 to 0.9 , and remains stable at $B S(0.1)$

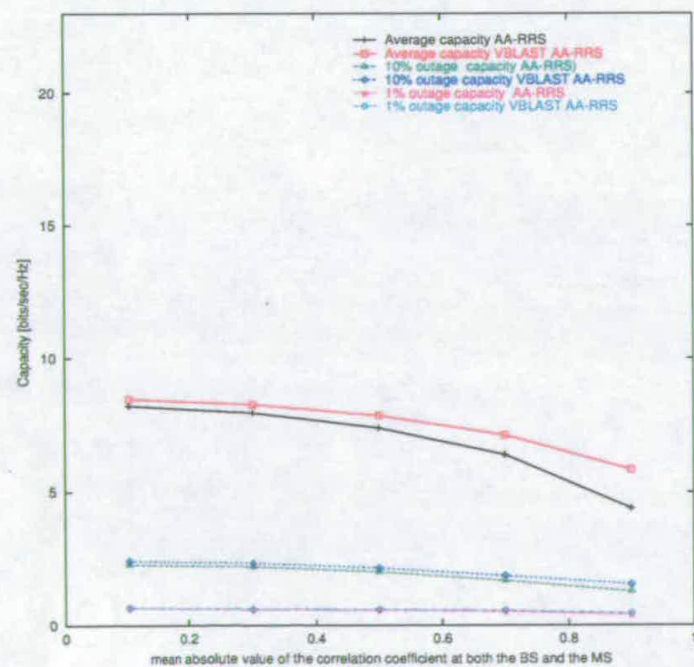

(a)

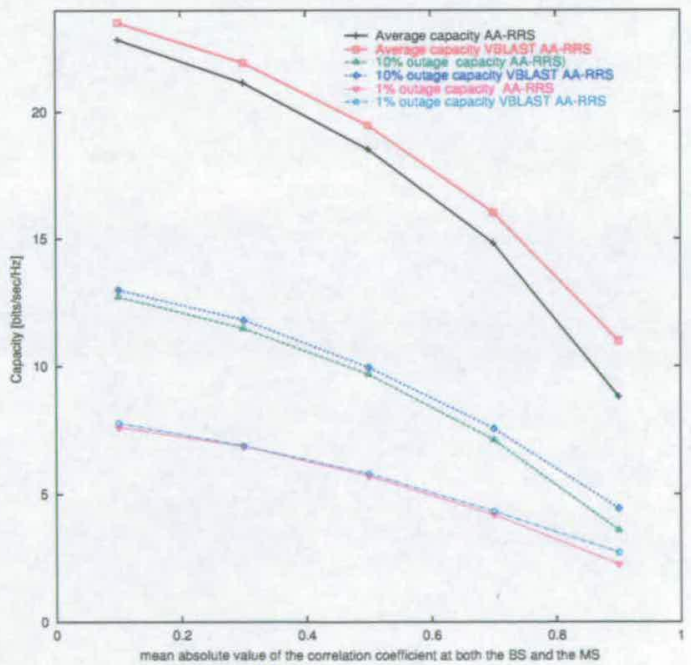

(b)

Figure 4.28: Effect of spatial correlation on MIMO systems of different number of transmit and receive antennas: (a) System capacities for a $(2,2)$ MIMO system, (b) System capacities for a $(6,6)$ MIMO system. For both cases, the correlation (at both the BS and MS) takes values from 0.1 to 0.9 . 
detection at the receivers.

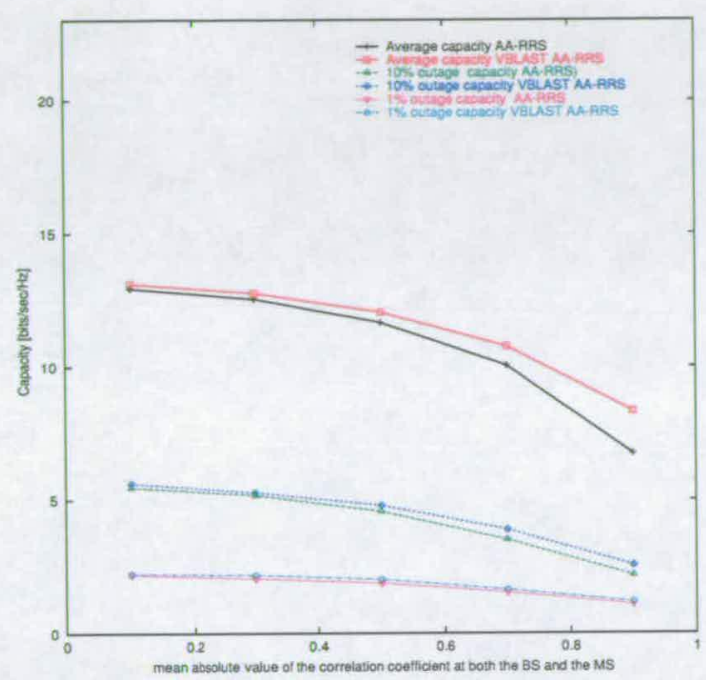

(a)

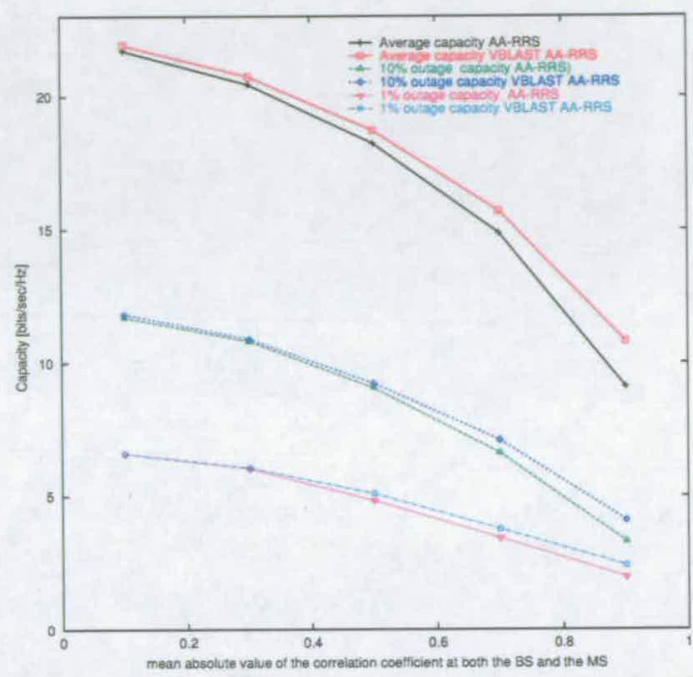

(b)

Figure 4.29: Effect of spatial correlation on MIMO systems of different number of transmit and receive antennas: (a) System capacities for a $(2,4)$ MIMO system, (b) System capacities for a $(4,6)$ MIMO system. For both cases, the correlation (at both the BS and MS) takes values from 0.1 to 0.9 .

\subsection{Conclusions}

In this chapter, we have analyzed a number of packet scheduling schemes based on round robin schedulers that may be used in a $\left(N_{T}, N_{R}\right)$ MIMO multiuser system. The basic functions of each algorithm have been introduced. Particularly, we propose three different packet scheduling algorithms :

- best user algorithm, implemented in AA-RRS best user scheme and VBLAST AA-RRS best user scheme.

- VBLAST detection, implemented in VBLAST AA-RRS scheme, VBLAST AA-RRS best user scheme and AA VBLAST fair scheme.

- AA (Antenna Assisted) fair algorithm, implemented in AA MMSE fair scheme and AA VBLAST fair scheme. 
Computer simulations have been conducted to compare the above scheduling schemes. We separate the results in two main categories, the overall efficiency and fairness results. Efficiency results are presented in terms of overall system capacity (average and outage capacities). fairness results are presented in terms of data rate per scheduled user and time delay per simultaneous user.

The analysis of these results shows that the employment of VBLAST detectors at the receivers has been proven to be beneficial for the system performance, especially in the case of correlated channels. Two points worth mentioning here. First each user $k$ may need to decode data for another user, which may require the use of encryption for security reasons. Second, we do not consider error-propagation effects on VBLAST performance. The VBLAST RRS scheme gives the highest average system capacity, but the VBLAST AA-RRS scheme is more efficient for delay sensitive systems. Also, the employment of the best user algorithm is proven to create better efficiency results (higher system throughput) but at the expense of fairness. The Antenna Assisted (AA) fair algorithm (AA MMSE fair and AA VBLAST fair) improves the system efficiency in terms of overall capacity and at the same time provides the same level of fairness among users for the time period of $\left\lceil\frac{K}{N_{T}}\right\rceil$ time slots, where $K$ the number of simultaneous users and $N_{T}$ the number of transmit antennas.

In conclusion, taking into account the simulation results, the AA VBLAST fair scheduling scheme is the most efficient scheduling scheme. Specifically, the AA VBLAST fair scheme provides higher system throughput than any other scheme without impacting the faimess among users. 


\section{Chapter 5 VBLAST Proportional Fair Scheme}

\subsection{Introduction}

In the previous chapter we investigated the downlink performance of some improved RRS schemes. The basic idea was to use multiple antennas to achieve diversity effect from multiple users and at the same time enhance this diversity gain by using the VBLAST detection method. In this chapter we will concentrate on the proportional fair (PF) scheduler and we will study the effect of the use of multiple antennas and VBLAST detection on the performance of the PF scheduler in the downlink channel.

In a general multiuser scenario, a user achieves higher data rates when the wireless channel conditions are better. Hence, the operation target of a scheduling scheme should be to take advantage of the fluctuations of channel conditions to achieve higher utilization of radio resources [87]. The performance (e.g., throughput) of a user depends on the channel condition it experiences. As a result we can have different performance when the same resource (e.g., radio frequency) is assigned to different users. For example, consider a cell with many users. Suppose that some users have good channel (they are close to the base station). On the other hand, a few users are at cell border, where these users experience a not so good radio channel, since there is interference from the adjacent cells and extra pathloss due to their distance from the base station. In this scenario, if we assume that the resources (power, time or codes) are assigned equally among the users the throughput of the users at the cell border will be much lower than the rest of the users.

Packet scheduling is very important in wireless systems, since the system performance depends directly on the scheduling decisions (allocation of the radio resources). In this section we study proportional faimess scheduling [36] and propose some new scheduling algorithms for MIMO systems based on this scheduling method. Proportional faimess can improve network throughput compared to round robin (Chapter 4 ) as follows. Consider a number of simultaneous users served by a cellular system. If we assume that the users experience time varying channel conditions, their performance is expected to be varying as well. The scheduling schemes choose 
which user should be transmitted to during a given TTI (Time Transmission Interval). Intuitively, we would serve users with better channel conditions so that the resource can be used efficiently. At the same time, we also want to provide some form of fairness or QoS guarantees to all users. For example, serving only users with good channels may result in very high spectral efficiency, but may lead other users to starvation [36]. This critical tradeoff motivates this work: to enhance the spectral efficiency by exploiting fluctuations of the channel conditions while at the same time meeting the requirements for faimess or QoS among the users.

In wireline systems, assignment of a specific amount of radio resource to a user, represents an assignment of a specific amount of data throughput. However, in wireless systems, the offered resource to a user and the achieved data rate of this user depend on the channel condition which varies over time. Therefore, we study two kinds of fairness: "effort" and "outcome" [142]. "Effort" faimess can be defined as the amount of radio resources (power, codes, allocation time) devoted to a user, whereas "outcome" is the throughput seen by the user. The basic purpose of scheduling is to serve users that offer the best channel conditions, but the problem is how long a user is willing to wait (for better conditions). In other words, there is a tradeoff between system performance gain and QoS performance. In this chapter we concentrate on proportional fair scheduling, aiming to improve its performance in terms of spectral efficiency (cell throughput) without to lower its QoS performance in terms of faimess among the users and time delay between subsequent transmissions to the same user. For this purpose, we employ MIMO arrays and VBLAST architecture, in order to combine the benefits of multiuser diversity (scheduling) and spatial diversity (multiple antennas and spatial multiplexing techniques). Also, note that throughout this chapter we assume a packet based system, as in Chapter 4.

The chapter is organized as follows. In section 5.2, we present the system channel models for a MIMO cellular system. In section 5.3 we discuss the PF (Proportional Fair) scheduling scheme and the related idea of multiuser diversity. In section 5.4 we present a few implementations of the PF scheme, specifically, we present AA-PF-MMSE single, AA-PF-MMSE multi, AA-PFVBLAST single, AA-PF-VBLAST multi and AA-PF-VBLAST multi fair schemes. Section 5.5 contains the simulation model and results, while section 5.6 contains our conclusions.

\subsection{System Model and Capacity}

We consider the downlink transmission of a single cell system comprising a base station and $K$ user terminals, as depicted in Figure 5.1. Our system model is much the same as the model 


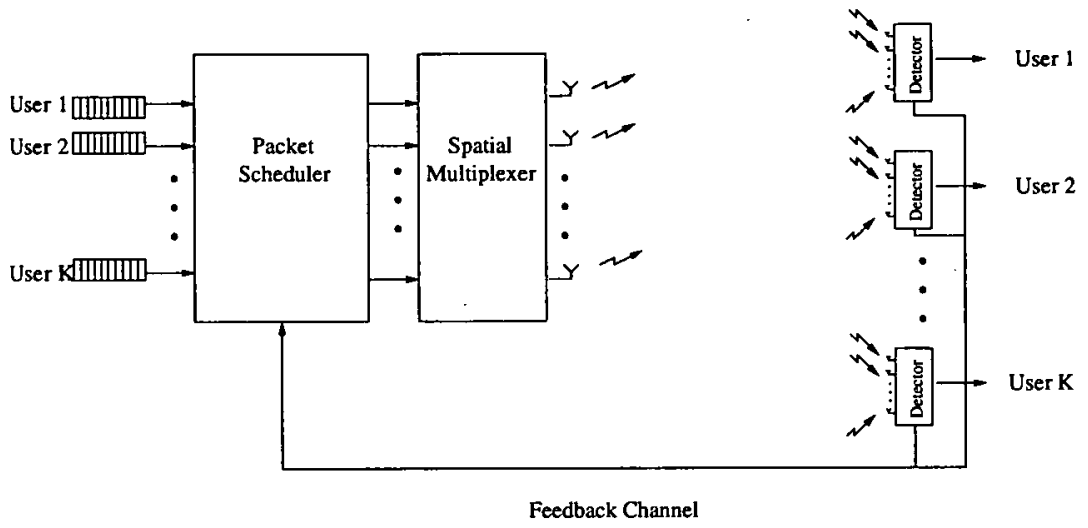

Figure 5.1: Downlink MIMO cellular system.

in chapter 4, section 4.2. The base station is equipped with $N_{T}$ transmit antennas, and each user terminal with $N_{R}\left(\geq N_{T}\right)$ receive antennas. The $K$ active users are served by the base station in a time division fashion, and these active users are distributed uniformly over the circular cell with radius $R$. The receiver of each user detects data packets intended for that user using either a minimum mean square error (MMSE) detector or a Vertical Bell Laboratories Layered Space-Time (VBLAST) detector. The receiver also estimates the post-detection signalto-interference-plus-noise ratio (SINR) for each transmit antenna, and passes this to the base station through an uplink feedback channel. The packet scheduler at the base station using this SINR information determines which packet to transmit through each transmit antennas. The channel matrix $\boldsymbol{H}_{k}(t)$ between the BS and the user $k$ for a time slot $t$ may be expressed as (same as in chapter 4 , equation 4.1 )

$$
\boldsymbol{H}_{k}(t)=\sqrt{S N R_{0}\left(r_{k} / R\right)^{-a_{D L}} 10^{S_{k}(t) / 10}} G_{k}(t)
$$

where $S N R_{0}$ denotes the median SNR at the cell boundary, $r_{k}$ is the distance between the BS and the user $k, a_{D L}$ is the path loss exponent, and $S_{k}(t)$ is a real Gaussian random variable with zero mean and variance of $\sigma_{S}^{2}$. An $N_{R} \times N_{T}$ matrix $G_{k}(t)$ represents Rayleigh-distributed multipath fading. At this point we have to refer to the notion of change in rate concerning the pathloss exponent and the shadowing effect. In contrast to the Rayleigh distributed multipath fading (expressed by the matrix $\boldsymbol{G}_{k}(t)$ ) which changes every time slot, the pathloss exponent (expressed by $r_{k}$ ) and the shadowing (expressed by the $S_{k}(t)$ term) do not change every time slot. In an average urban scenario the change rate of the pathloss and shadowing effect on the 
physical channel is much slower than the change rate of the Rayleigh fading (which changes every time slot). In practice, we can assume that the pathloss exponent and the shadowing change every 2000 time slots. In the rest of this work we will assume that these terms change every 2000 time slots. Note that these assumptions for pathloss and shadowing do not change the capacity results of Chapter 4 .

\subsubsection{Capacity}

For the system model described in previous section the system capacity for the time slot $t$ may be calculated as [137]

$$
\left.C_{M I M O}\left(t \mid\left(k_{1}, k_{2}, \ldots, k_{N_{T}}\right)\right)=\sum_{n=1}^{N_{T}} \log _{2}\left(1+\gamma_{k_{n}, n}\right)\right)
$$

where $\gamma_{k_{n}, n}$ denotes the post detection SINR for the channel between the $n$th transmit antenna and the $k_{n}$ th user, as expressed in (4.6) after MMSE nulling. In the case of VBLAST detection and MMSE nulling the post detection SINR $\gamma_{k_{n}, n}$ is given by equation (4.8). The sequence $\left(k_{1}, k_{2}, \ldots, k_{N_{T}}\right)$ represents a mapping between transmit antennas and users, with $k_{n}$ denoting the user index assigned to the $n$th transmit antenna.

\subsection{Proportional Fair Algorithm}

This algorithm was firstly described in [89] and further analyzed in [85], [96], [99], [101]. According to [36], the proportional fair scheduler at each scheduling instant serves the user $i$ with the largest instantaneous channel quality $D R C_{i}(t)$ relative to the average data rate $R_{i}(t)$ :

$$
k(t)=\underset{i \in\{1,2, \cdots, K\}}{\arg \max } \frac{D R C_{i}(t)}{R_{i}(t)}
$$

where $k(t)$ denotes the user index to be served at the $t$ th time slot, $D R C_{i}(t)$ is the instantaneous data rate experienced by user. $i$ if it is served by the Packet Scheduler, and $R_{i}(t)$ is the average data rate (or average throughput) received by the mobile over a window of appropriate size.

In real systems, packet scheduling is constrained by the maximum application-delay tolerance, though this delay requirement is much more relaxed for packet data services than for voice 
or streaming video services [78]. Data packet transfers include applications such as HTTP, FTP, email, and database backup. Such type of transfers tend to have zero tolerance for packet loss, and high tolerance for delay. On the other hand, video/voice streaming includes real time applications such as video conference or voice communication products. Video/voice streaming tend to have low tolerance for packet loss, but much stricter tolerance for delay. The scheduling scheme must also consider the fairness among multiple users for the service. The proportional fair scheme [89] [36] aims to help this issue. The proportional fair scheme exploits temporal variations of the channel conditions in the scheduling decision. This algorithm serves a user when the instantaneous relative channel quality outperforms the one from the remaining users in the cell. The instantaneous channel condition of the $k$ th user at the $t$ th time slot can be represented as the supportable data rate $R_{i}(t)$ fed back from the user. Using the supportable rates of users, the PF scheduling decision at the time slot $t$ may be expressed as in equation (5.3) [36]. $R_{i}(t)$ is an estimate of the average supportable rate of the $i$ th user, and it is obtained using a low pass filter with a time constant of $t_{c}$ slots as [36]

$$
\begin{gathered}
R_{i}(t)=\left(1-1 / t_{c}\right) R_{i}(t-1), \\
R_{k}(t)=\left(1-1 / t_{c}\right) R_{k}(t-1)+\left(1 / t_{c}\right) D R C_{k}(t)
\end{gathered} \quad i \neq k
$$

where $D R C_{k}(t)$ represents the user data rate in the present TTI (transmission time intervals) $t$. Equation (5.4) is updated every TTI with a user data rate $D R C_{k}(t)$ equal to zero if the user is not served, and with a data rate equal to the transmitted bits divided by the TTI duration if the user is scheduled and successfully receives his packet. The averaging length $t_{c}$ should be set long enough to ensure that the process averages out the fast fading variations, but short enough to still reflect medium term conditions such as the shadow fading [144].

For reasons of simplicity, the traffic model employed in this work does not include any traffic inactivity (i.e. the infinite buffer model) [145]. In the case of a bursty traffic model, during the periods the user does not have data to transmit, the user throughput decreases. This reduction of the user throughput during the traffic inactivity periods artificially increases the priority computation of the proportional fair algorithm (equation (5.3)). In order to avoid that the burstiness influences the priority computations, the stochastic approximation of the user throughput (equation (5.4)) should only be updated when the users have data queued for transmission (or when they are served). In our model though we will always use the equation (5.4) and update it for all time instants. 


\subsubsection{Multiuser Diversity}

The basic concept of diversity is: transmit the signal via several independent diversity branches to get independent signal replicas. Traditional forms of diversity include time, frequency and antennas. Multiuser diversity arises from independent fading channels across different users [79]. This fact could be exploited by a scheduling scheme. In particular, due to the time shared nature of the transmission model, the packet scheduler can serve at any instant the user with the most favourable channel quality characteristics, thereby introducing a degree of selection (multi-user) diversity, with benefit for the user and/or system throughput. In this work we investigate the improvement from antenna diversity (multiple antennas) and multiuser diversity. It has been proven by Jiang [78], that in a data network of multiple users, multiuser diversity can greatly increase system throughput. Motivated by Tse [79], one approach to increase the throughput of multi user systems is to use multiuser diversity to take advantage of the independence of the fading statistics of different users. In this chapter we will investigate the performance improvements offered by the application of multiuser diversity to spatial multiplexing systems (MIMO antennas). Specifically we study the interaction of the proportional fair scheduling scheme with spatial multiplexing techniques (MIMO arrays and VBLAST detection).

\subsection{Proportional Fairness Scheduling Schemes Variants}

In this chapter a few variants of the proportional fair scheduling scheme have been implemented. These algorithms are presented in the subsequent subsections. All these schemes are based on the proportional fair scheme as presented by Jalali in [36].

\subsubsection{Antenna Assisted Proportional Fairness MMSE single (only one user each time slot)}

The antenna assisted proportional fairness MMSE single (AA-PF MMSE single) scheduling scheme is a straight forward implementation of the PF scheme in the case of MIMO wireless links. Originally the PF scheme has been proposed for SISO (single-input single-output) and SIMO (single-input multiple-output) systems ([36]). In this section we present an PF scheduling scheme for the case of a MIMO cellular system as described in section 5.2. When multiple input and multiple output systems achieve spatial multiplexing, multiple spatial links are cre- 
ated at each time slot [136]. Each spatial link consists of those formed between the $N_{T}$ transmit antennas and $N_{R}$ receive antennas of each user. Different information symbols can be transmitted through these multiple spatial links during the same time slot. As a result, the data rate $D R C_{i}$ of user $i$ is represented by the sum of the data rates of the $N_{T}$ spatial links for that user

$$
D R C_{i}(t)=\sum_{n=1}^{N_{T}} R_{k, n}(t)
$$

where $R_{k, n}(t)$ denotes the data rate of the $n$th spatial channel for the $k$ th user at the $t$ th time slot.

In the AA-PF single scheme (like all PF schemes) the scheduler selects the mobile with the highest ratio $D R C_{i}(t) / R_{i}(t)$. This can be expressed as

$$
k(t)=\underset{i \in\{1,2, \cdots, K\}}{\arg \max } \frac{D R C_{i}(t)}{R_{i}(t)}
$$

where $k(t)$ denotes the user selected to be served at the $t$ th time slot, $D R C_{i}(t)$ is the instantaneous data rate experienced by user $i$ if it is served by the Packet Scheduler, and $R_{i}(t)$ is the average data rate (or average throughput) received by the mobile over a window of an appropriate time duration. The computation of the user throughput $R_{i}(t)$ is given from the following equation

$$
\begin{gathered}
R_{i}(t)=\left(1-1 / t_{c}\right) R_{i}(t-1), \\
R_{k}(t)=\left(1-1 / t_{c}\right) R_{k}(t-1)+\left(1 / t_{c}\right) D R C_{k}(t)
\end{gathered} \quad i \neq k
$$

where $D R C_{k}(t)$ is given from the following equation in the case of a MIMO multiuser system, where each receiver is equipped with MMSE detector.

$$
D R C_{k}^{A A-P F \text { single }}(t)=\sum_{n=1}^{N_{T}} \log _{2}\left(1+\gamma_{k, n}(t)\right)
$$

where $\gamma_{k, n}$ denotes the post-detection SINR for the channel corresponding to the $n$th transmit antenna and the $k$ th user. The post-detection SINR is defined as the SINR of a transmit symbol 
after MMSE nulling, and it may be expressed as [141]

$$
\gamma_{k, n}(t)=\frac{P_{R}\left|\left[W_{k}(t) H_{k}(t)\right]_{n n}\right|^{2}}{\sigma_{N}^{2} N_{T} \sum_{m=1}^{N_{T}}\left|\left[W_{k}(t)\right]_{n m}\right|^{2}+P_{R} \sum_{m=1, m \neq n}^{N_{T}}\left|\left[W_{k}(t) H_{k}(t)\right]_{n} m\right|^{2}}
$$

where $P_{R}$ is the total received signal power, and $\sigma_{N}^{2}$ is the noise power per received antenna. The nulling weight matrix $W_{k}(t)$ for the MMSE is given as [141]

$$
\boldsymbol{W}_{k}(t)=\boldsymbol{H}_{k}^{H}(t)\left(\boldsymbol{H}_{k}(t) \boldsymbol{H}_{k}^{H}(t)+\left(\sigma^{2} N_{T} / P_{R}\right) \boldsymbol{I}_{N_{R}}\right)^{-1}
$$

where $(\bullet)^{H}$ denotes the conjugate transpose, and $\boldsymbol{I}_{N_{R}}$ is the $N_{R} \times N_{T}$ identity matrix.

Specifically, the flowchart for the scheme is shown in Figure 5.2. Also, the pseudocode is given in appendix B (Figure B.1).

The computational complexity of AA-PF MMSE single scheme is studied as a function of the number of active users $K$, and the number of transmit antennas $N_{T}$ (we assume that $N_{T}=N_{R}$ ). The algorithm first performs $K$ iterations of the MMSE algorithm to calculate $\gamma_{k_{n}, n}$ between the $N_{T}$ transmit antennas and $K$ users of the SUG. The MMSE algorithm has a complexity order of $\mathrm{O}\left(N_{T}^{3}\right)$. After the calculation of the post-detection SNR values, the algorithm requires $K$ comparisons to find the mapping sequence that maximizes the system capacity. Summarizing, the computational complexity of the AA-PF MMSE single scheme is $\mathrm{O}\left(K N_{T}^{3}+K\right)$.

\subsubsection{Antenna Assisted Proportional Fairness VBLAST single (only one user each time slot)}

The Antenna Assisted Proportional Fairness VBLAST single (AA-PF-VBLAST single) scheme is based on the AA-PF-MMSE single scheme (described above). The basic change is the use of VBLAST detectors at the receivers instead of the MMSE detectors used in AA-PFMMSE scheme. The AA-PF-VBLAST scheduling procedure starts with the application of the VBLAST detection at the receivers of all users and the computation of the SINR values using equation (4.8). Then, the scheduler assigns all the antennas to the user with highest $D R C_{i} / R_{i}$ ratio (as in the equation (5.6)).

The flowchart of the AA-PF-VBLAST single algorithm is shown in Figure 5.3. The pseudocode is presented in appendix A (Figure B.2). 


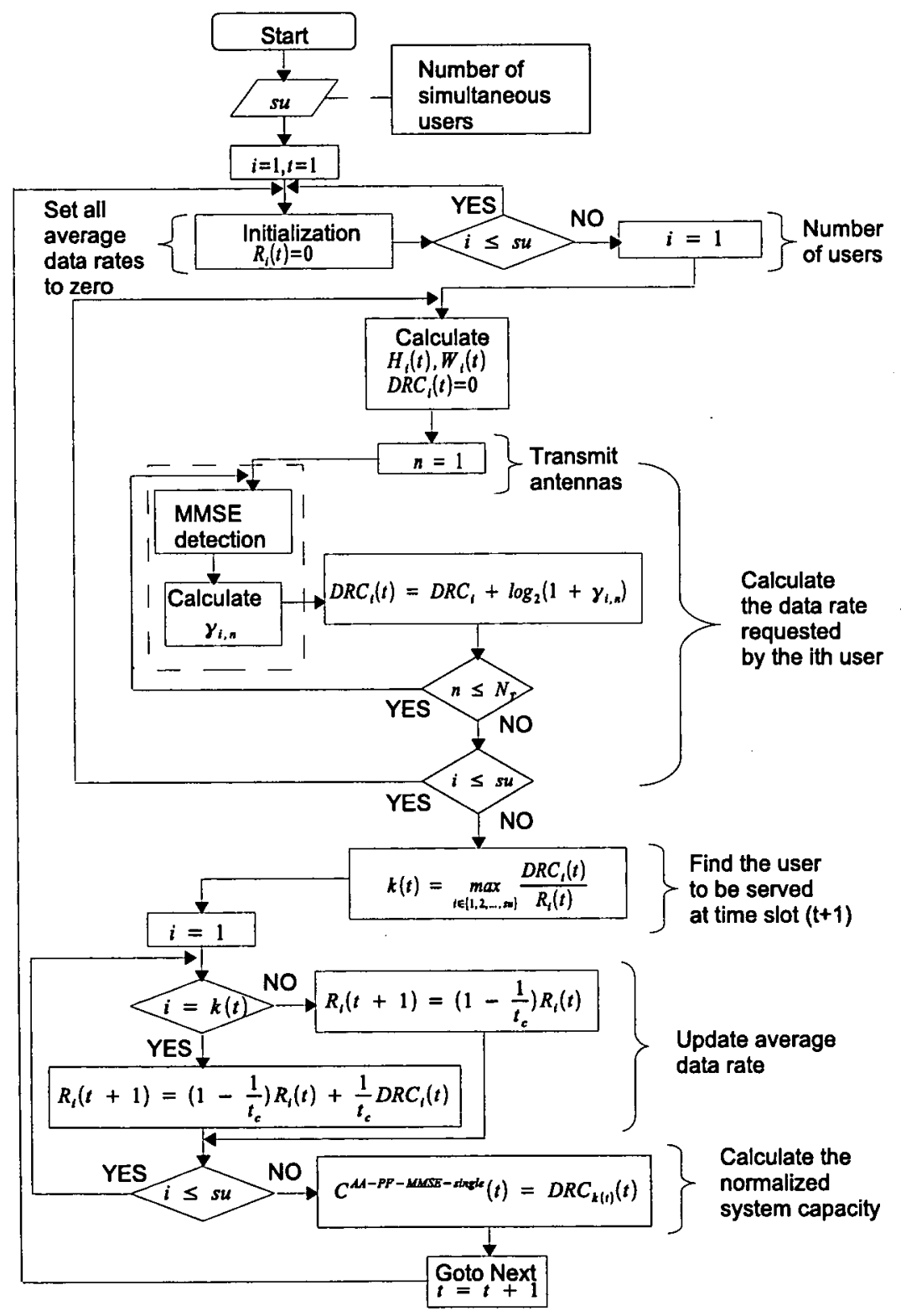

Figure 5.2: $A A-P F-M M S E$ single algorithm 
The computational complexity of AA-PF VBLAST single scheme is studied as a function of the number of active users $K$, and the number of transmit antennas $N_{T}$ (we assume that $N_{T}=N_{R}$ ). The algorithm first performs $K$ iterations of the VBLAST algorithm to calculate $\gamma_{k_{n}, n}$ between the $N_{T}$ transmit antennas and $K$ users of the SUG. The VBLAST algorithm has a complexity order of $\mathrm{O}\left(N_{T}^{4}\right)$. After the calculation of the post-detection SNR values, the algorithm requires $K$ comparisons to find the mapping sequence that maximizes the system capacity. Summarizing, the computational complexity of the AA-PF VBLAST single scheme is $\mathrm{O}\left(K N_{T}^{4}+K\right)$.

\subsubsection{Antenna Assisted Proportional Fairness multi (more than one user each time slot)}

The AA-PF-MMSE single scheduling scheme in section 5.4.1 allocates all $N_{T}$ spatial links to one user, so that during each time slot only one user is served. However, in multiple input and multiple output systems based on spatial multiplexing, each spatial link or transmitter could be allocated to more than one users each time slot. Exploiting this advantage by packet scheduling is the basic idea of the Antenna Assisted Proportional Fairness multi scheduling algorithm (AA$\mathrm{PF}$ multi). In particular, we aim to enhance the performance of the PF scheme by combining the multiuser diversity offered by the PF scheduler and spatial diversity offered by the MIMO antenna technology. Multiuser diversity is achieved by exploiting the independence of the the fading statistics of different users by selecting the simultaneous user with the best channel conditions [136]. On the other hand spatial diversity is achieved through antenna diversity by using MIMO antenna arrays [78]. In the AA-PF multi scheme, transmit antennas are allocated to more than one different users at each time slot, and the scheduling process is performed in $N_{T}$ sequential stages. At each stage, one transmit antenna is allocated to the user that experiences the best channel conditions in the proportional fair sense, and the average data rates of users are calculated according to the allocation decisions. The algorithm is summarized below.

The scheduler assigns the mobile to the antenna that gives the highest ratio $D R C_{i, n}(t) / R_{i, n}(t)$. This can be expressed as:

$$
k_{n}(t)=\underset{i \in\{1,2, \cdots, K\}}{\arg \max } \frac{D R C_{i, n}(t)}{R_{i, n}(t)}
$$

where $k_{n}(t)$ denotes the user index selected to be assigned to the $n$th transmit antenna at the $t$ th time slot, $D R C_{i, n}(t)$ is the instantaneous data rate experienced by user $i$ if it is assigned to $n$th transmit antenna, and $R_{i, n}(t)$ is the average data rate (or average throughput) transmitted 


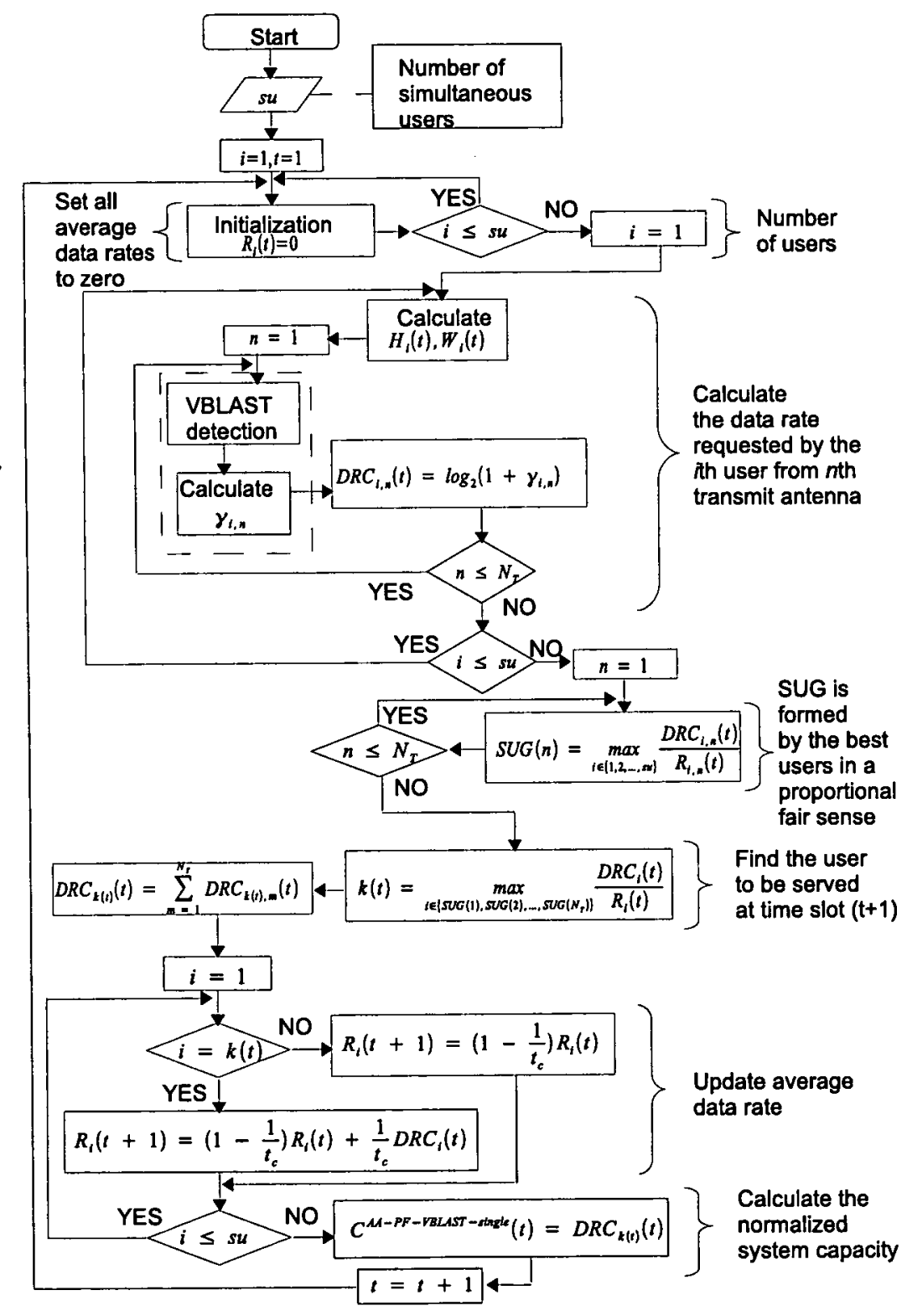

Figure 5.3: $A A-P F-V B L A S T$ single algorithm 
by the $n$th antenna and received by the mobile user $i$ over a window of appropriate size. The computation of the user throughput $R_{i, n}(t)$ is given from the following equation

$$
\begin{array}{cc}
R_{i, n}(t)=\left(1-1 / t_{c}\right) R_{i, n}(t-1), & i \neq k \\
R_{k, n}(t)=\left(1-1 / t_{c}\right) R_{k, n}(t-1)+\left(1 / t_{c}\right) D R C_{k, n}(t) &
\end{array}
$$

where $D R C_{k, n}(t)$ is given from the following equation in the case of a MIMO multiuser system, where each receiver is equipped with MMSE detector.

$$
D R C_{k, n}^{A A-P F} \text { multi }^{(t)}=\log _{2}\left(1+\gamma_{k, n}(t)\right)
$$

where $\gamma_{k, n}$ denotes the post-detection SINR for the channel corresponding to the $n$th transmit antenna and the $k$ th user. The post-detection SINR is defined as the SINR of a transmit symbol after MMSE nulling and it can be computed using equation (5.9).

It should be noted that the AA-PF multi algorithm demands $N_{T}$ times more computations than the AA-PF single scheme but offers higher degree of faimess among the simultaneous users. The flowchart of the AA-PF multi scheduling algorithm is drawn in Figure 5.4. The pseudocode is shown in appendix B (Figure B.3).

The computational complexity of AA-PF MMSE multi scheme is studied as a function of the number of active users $K$, and the number of transmit antennas $N_{T}$ (we assume that $N_{T}=N_{R}$ ). The algorithm first performs $K$ iterations of the MMSE algorithm to calculate $\gamma_{k_{n}, n}$ between the $N_{T}$ transmit antennas and $K$ users of the SUG. The MMSE algorithm has a complexity order of $\mathrm{O}\left(N_{T}^{3}\right)$. After the calculation of the post-detection $\mathrm{SNR}$ values, the algorithm requires $N_{T}$ iterations, with $K$ comparisons in each iteration to find the best users in a proportional fair sense to form the SUG. Summarizing, the computational complexity of the AA-PF MMSE multi scheme is $\mathrm{O}\left(K N_{T}^{3}+K N_{T}\right)$.

\subsubsection{Antenna Assisted Proportional Fairness VBLAST multi (more than one user each time slot)}

The Antenna Assisted Proportional Fairness VBLAST multi (AA-PF-VBLAST multi) scheme is based on the AA-PF multi scheme (section 5.4.3). The basic difference is the use of VBLAST [131] detectors at each receiver. Similarly to Chapter 4, the main idea of using VBLAST de- 


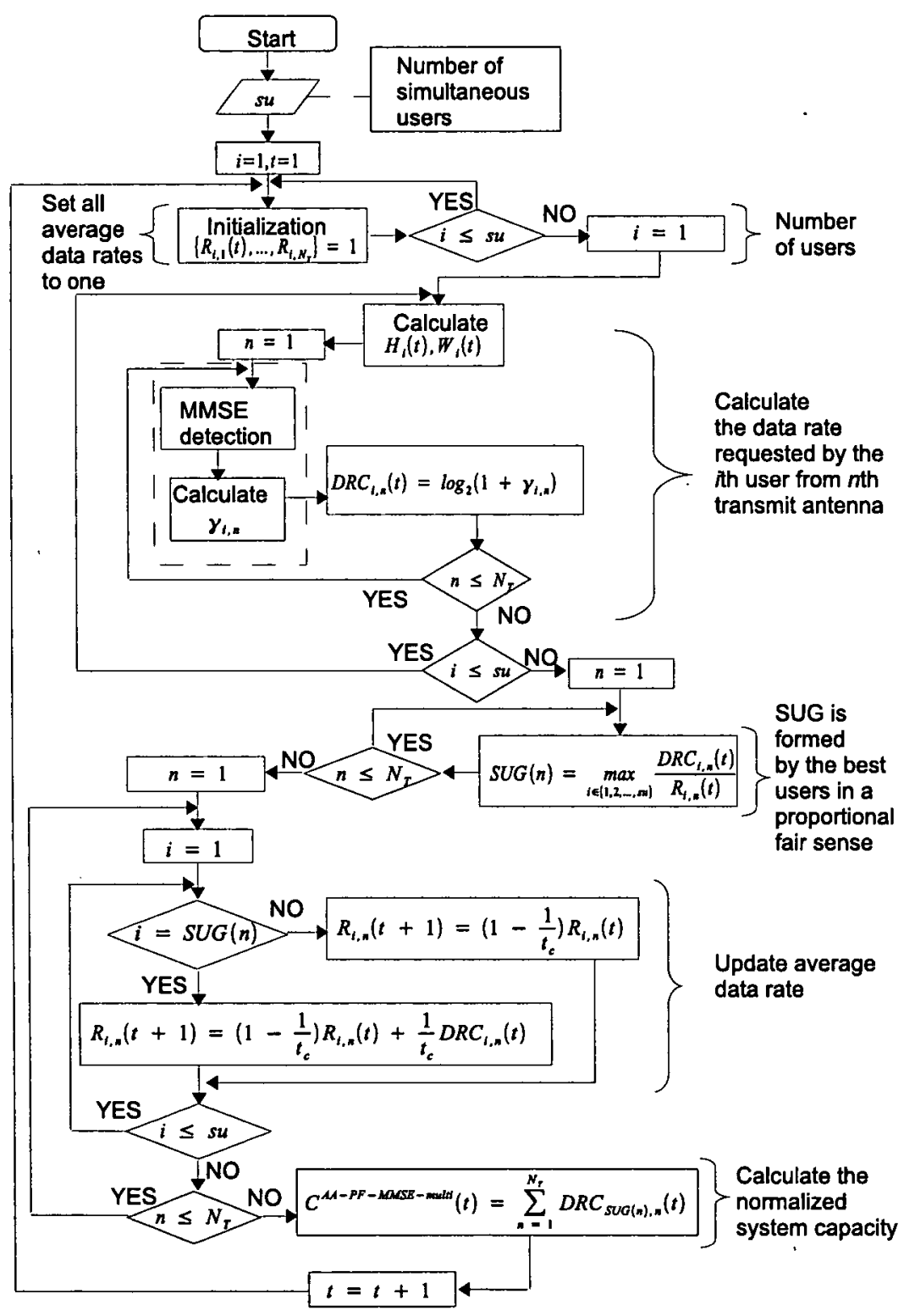

Figure 5.4: $A A-P F-M M S E$ multi algorithm 
tection is the feature of the symbol cancellation. Using symbol cancellation, interference from already-detected components of the transmitted signal are subtracted out from the received signal vector, resulting in a modified receiver vector in which fewer interferers are present. When nulling with symbol cancellation are used together (VBLAST) the system performs better than in the case of pure nulling [131]. The algorithm can be executed following the steps described in section 5.4.3 (equations (5.11)-(5.13)). There are only two differences.

First, in equation (5.13) the post-detection SINR of a transmit symbol after VBLAST detection with MMSE nulling can be computed using the following equation

$$
\gamma_{k, n}(t)=\frac{P_{R}\left|\left[W_{k}(t) H_{k}(t)\right]_{n n}\right|^{2}}{\sigma_{N}^{2} N_{T} \sum_{m=1}^{N_{T}}\left|\left[W_{k}(t)\right]_{n m}\right|^{2}+P_{R} \sum_{m=1, m \neq n, m \notin D_{k, n}(t)}^{N_{T}}\left|\left[W_{k}(t) \boldsymbol{H}_{k}(t)\right]_{n m}\right|^{2}}
$$

this equation has been explained thoroughly in Chapter 4 section 4.4.1. Another point to be noted is that the data rates have to be feasible (Section 4.4.2), because of the use of VBLAST detection. In short, the assumed data rates have to be smaller than the capacities of the wireless links. In the VBLAST algorithm, a signal must be detected successfully for it to be subtractively cancelled. This constraint is satisfied by the application of the feasibility sest (Section 4.4.2, Figure 4.6).

The general AA-PF-VBLAST multi scheduling algorithm, that has been simulated in this work, may be expressed as in Figure 5.6. Also the pseudocode for this scheme is given in appendix B (Figure B.4). For simulation performance reasons, the simulated algorithm does not apply VBLAST detection to all the simultaneous users each time slot, as that would be too computationally intensive. Specifically, each time slot we first apply MMSE detection for all the users $K$. The scheduler creates the SUG (Scheduled User Group, section 4.3.2) choosing the $N_{T}$ users with the highest $D R C_{i, n} / R_{i, n}$, computing the SINR values using equation (5.9) (exactly as in section 5.4.3). As soon as the SUG is formed, we apply VBLAST detection at receivers of the users that have been chosen to form the SUG. Finally, the scheduler assigns the users to the antennas that give the highest $D R C_{i, n}(t) / R_{i, n}(t)$ ratios at the time slot $t$ :

$$
k_{n}(t)=\underset{i \in\left\{S U G(1), S U G(2), \cdots, S U G\left(N_{T}\right)\right\}}{\arg \max } \frac{D R C_{i, n}(t)}{R_{i, n}(t)}
$$




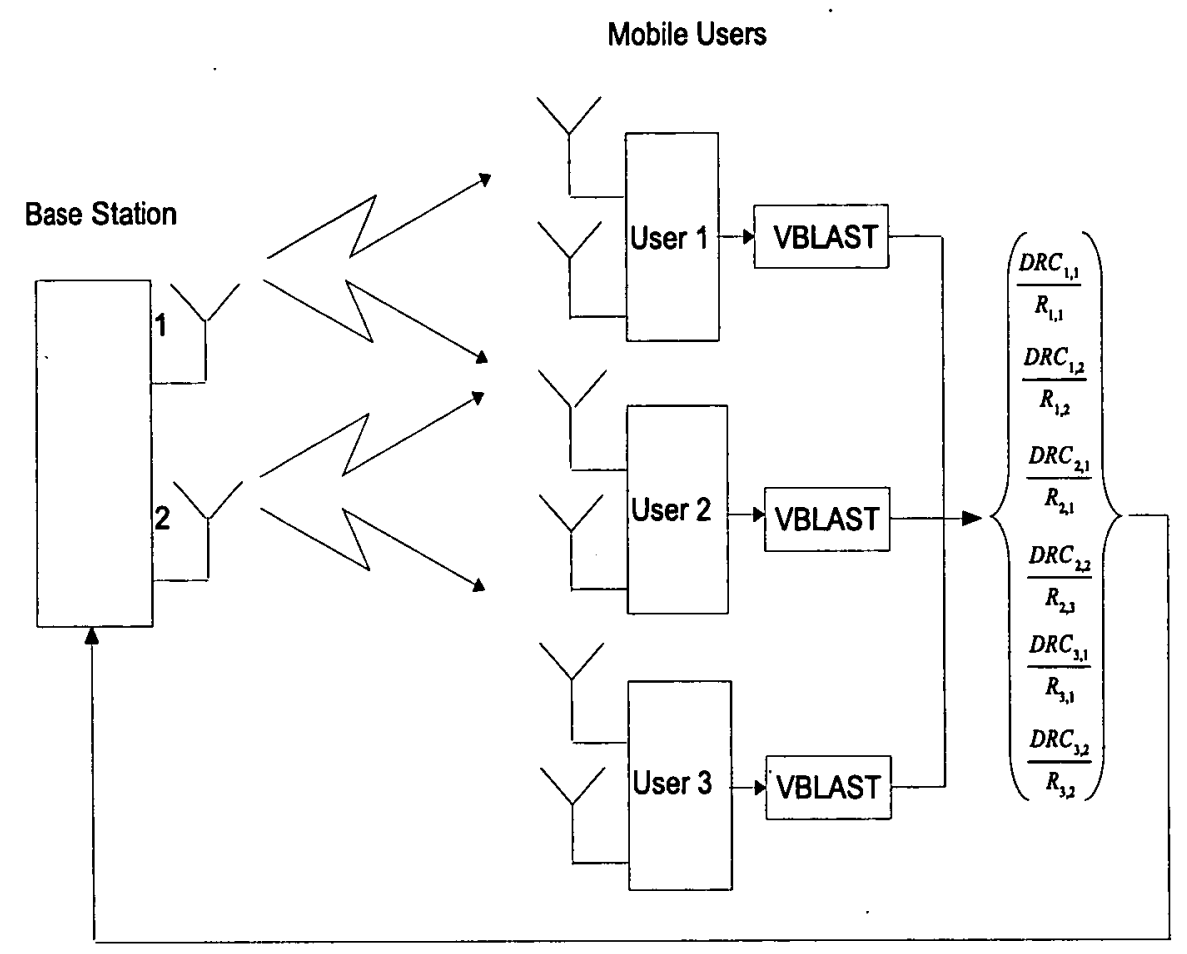

Figure 5.5: $A A-P F-V B L A S T$ multi scheme - example 
In Figure 5.5, an example of the implementation of the AA-PF-VBLAST multi scheme is depicted. In this MIMO $(2,2)$ system there are 3 simultaneous users. After VBLAST detections at each mobile users the values for the ratio $D R C_{i, n} / R_{i, n}$ are calculated and sent back to the base station through the feedback channel. Using these ratios the scheduler creates the SUGs selecting the mappings that give the highest $D R C_{i, n} / R_{i, n}$.

Similarly to the AA-PF MMSE multi scheme, the computational complexity of the AA-PF VBLAST multi scheme is $\mathrm{O}\left(K N_{T}^{4}+K N_{T}\right)$, where $N_{T}^{4}$ represents the VBLAST detection.

\subsubsection{Antenna Assisted Proportional Fairness VBLAST multi fair (more than one users each time slot - but users not allowed to be assigned to multiple antennas)}

Antenna Assisted Proportional Faimess VBLAST multi fair (AA-PF-VBLAST multi fair) is based on the AA-PF-VBLAST multi (section 5.4.4). The difference is that in AA-PF-VBLAST multi fair scheme, one user is not allowed to be assigned to multiple antennas. This constraint is meant to improve the fairness among the users, allowing more users to be served in each time slot. According to AA-PF-VBLAST multi scheme, the scheduler assigns to each antenna the best user in the proportional fair sense (for the spatial channel between this user and the corresponding transmit antenna). In practice for every time slot, there is always one user that is closer to the base station and this user establishes the best channels between himself and most of the base station's transmit antennas. This phenomenon leads the scheduler to assign the most of the antennas to same user. This is why the AA-PF-VBLAST multi fair scheduler does not allow more than one transmit antenna to be allocated to the same user. The AA-PF-VBLAST multi fair algorithm is the same as the AA-PF-VBLAST multi (section 5.4.4), apart from the constraint that each time slot no more than one antenna may be assigned to the same user:

$$
k_{n}(t)=\underset{\substack{i \in\left\{S U G(1), S U G(2), \cdots, S U G\left(N_{T}\right)\right\} \\ i \notin\left\{k_{1}, k_{2}, \cdots, k_{n-1}\right\}}}{\arg \max } \frac{D R C_{i, n}(t)}{R_{i, n}(t)}
$$

The computational complexity of the AA-PF VBLAST multi fair scheme is the same as that of AA-PF VBLAST multi scheme, $\mathrm{O}\left(K N_{T}^{4}+K N_{T}\right)$. 


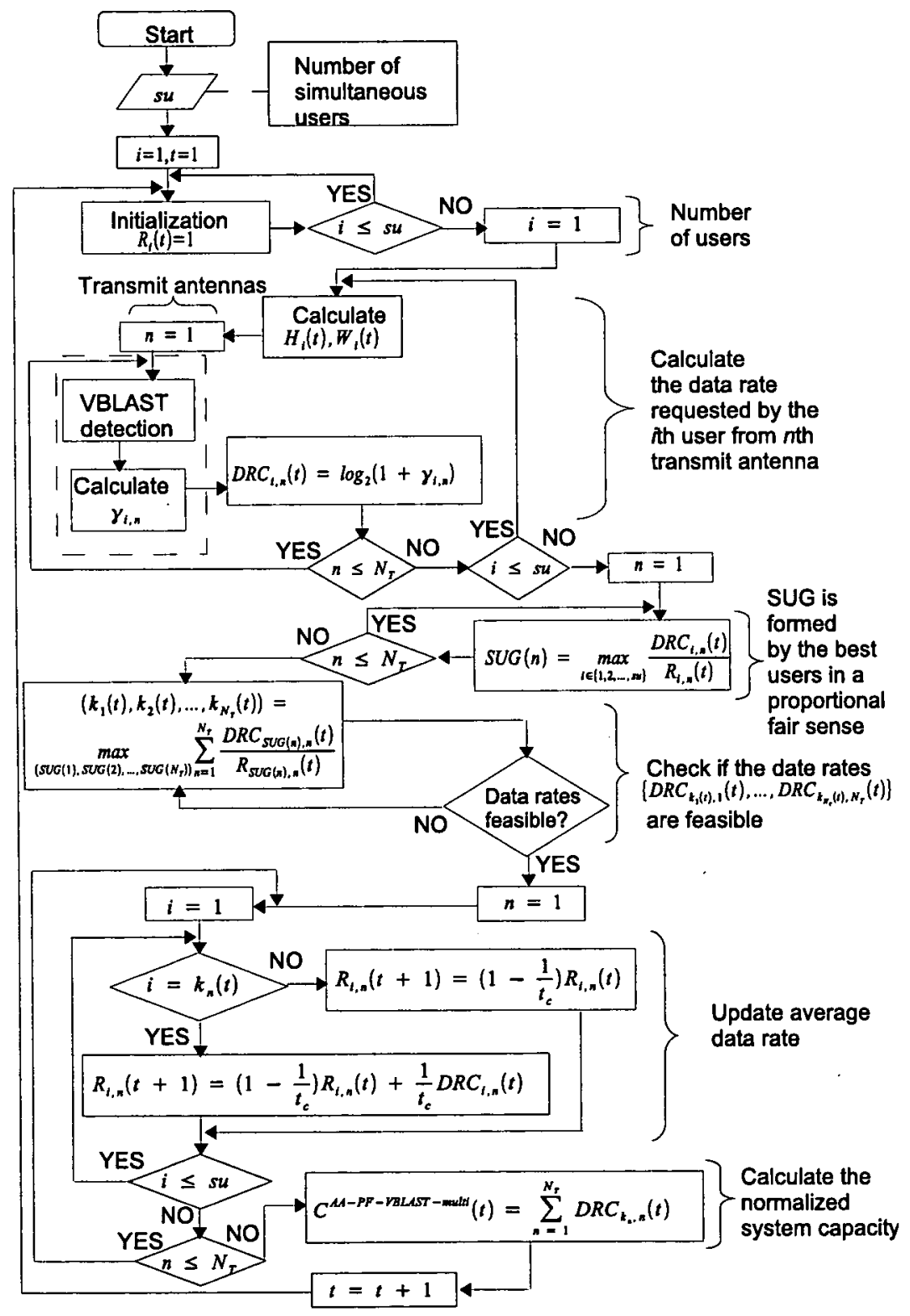

Figure 5.6: $A A-P F-V B L A S T$ multi algorithm 


\subsection{Simulation}

In this section, the performance of the AA-PF single, AA-PF multi, AA-PF-VBLAST single and AA-PF-VBLAST multi, AA-PF-VBLAST multi fair scheduling schemes is evaluated and compared with one another in terms of the system capacity (throughput), the fairness and the time delay. Also the capacity performance of selected scheduling schemes from Chapter 4 (VBLAST AA-RRS and AA VBLAST fair) is compared with scheduling schemes from Chapter 5. The system capacity is normalized by the system bandwidth. For the AA-PF single, AA-PF multi, AA-PF-VBLAST single and AA-PF-VBLAST multi, AA-PF-VBLAST multi fair scheduling schemes, the system capacities are given from equation (5.5) using 20,000 random realizations of the channel matrix in equation (5.1). The $R_{k, n}$ values used in equation (5.5) are calculated using equation (5.9) for the case of AA-PF single and AA-PF multi and the equation (4.8) is used in the case of AA-PF-VBLAST single and AA-PF-VBLAST multi, AA-PF-VBLAST multi fair scheduling schemes. The system capacity is evaluated in both the average sense and outage sense. The average capacity sets an upper bound for the achievable throughput and outage capacity and is a very important performance measure for applications with delay constraint [22]. The average capacity is defined as the capacity averaged over all possible channel realizations, and the $x \%$ outage capacity is defined such that the probability of the capacity at a time slot being less than the value is $x \%$. The path loss exponent $a_{D L}$ and log standard deviation of shadow fading $\sigma_{s}$ in (5.1) are assumed to be 3.7 and $8 \mathrm{~dB}$, respectively. We have used these specific values for path loss and shadowing, because these values give us a good match with the results provided by Oh-Soon Shin and Kwang Bok Lee [137].

\subsubsection{Results}

In this section, the performances - in terms of system capacities and time delays - of different scheduling schemes are evaluated and compared with each other. Also, results of different scheduling schemes are presented in terms of time delay and data rate per scheduled user. We separate the results into four subsections according to the following parameters.

1. Scheduling schemes throughput (AA-PF single, AA-PF multi, AA-PF-VBLAST single and AA-PF-VBLAST multi, AA-PF-VBLAST multi fair, VBLAST AA-RRS and AA VBLAST fair).

2. Effect of number of transmit and receive antennas on system throughput. 
3. Time delay per simultaneous user.

4. Effect of spatial fading complex correlation on system throughput.

We will now discuss each category of results in turn.

\subsubsection{Scheduling Schemes}

In this section we measure the performance of various scheduling algorithms presented in this chapter. The platform for all the simulations in this section is a $(4,4)$ spatially uncorrelated MIMO system. We use the notation $\left(N_{T}, N_{R}\right)$ to denote a MIMO system with $N_{T}$ transmit antennas and $N_{R}$ receive antennas.

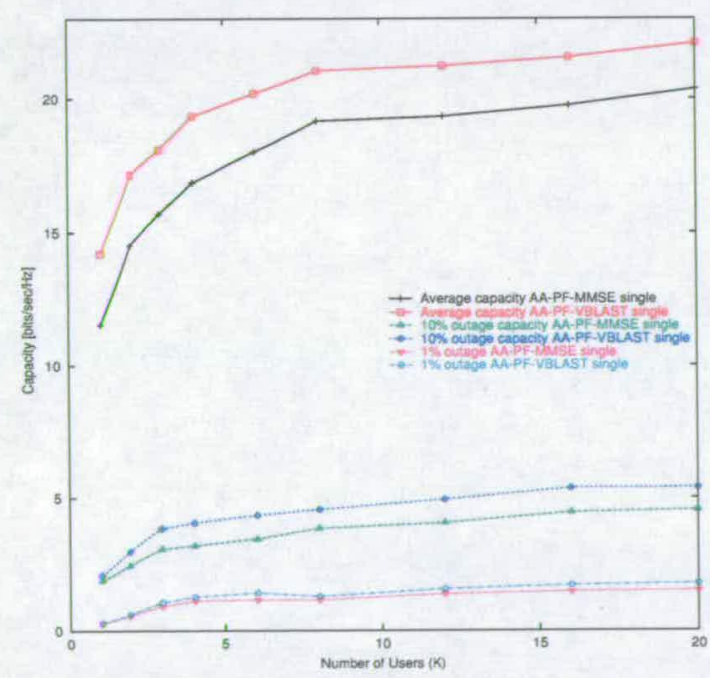

(a)

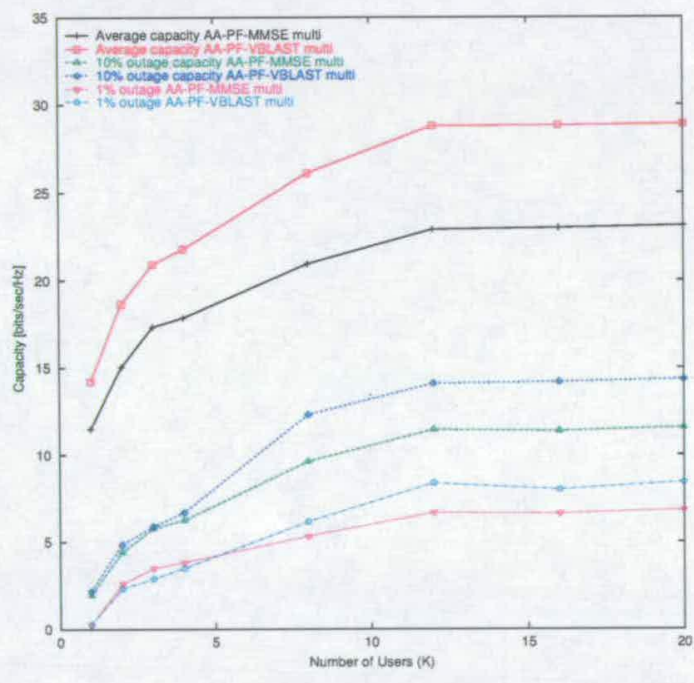

(b)

Figure 5.7: System capacities on a (4,4) MIMO system (SNR at boundary =OdB): (a) AA-PF-MMSE single vs AA-PF-VBLAST single, (b) AA-PF-MMSE multi vs AA-PF-VBLAST multi

\section{MMSE detection vs VBLAST detection}

The detection method that is employed at the receivers, plays important role in system performance. Figures 5.7(a) and (b) show an improvement in terms of system capacity due to the use of VBLAST detectors for a $(4,4)$ MIMO system. The transmit power is fixed to give $S N R_{0}=0 d B\left(S N R_{0}\right.$ denotes the median SNR power at the cell boundary). Figure 5.7(a) investigates the performance of the AA-PF single scheme for the case of MMSE detection and for 
the case of VBLAST detection. Specifically, for 20 users, there is a gain on average capacity of about $9 \%$ due to VBLAST detection over MMSE detection. In terms of outage capacities, the gain due to VBLAST detection for the $10 \%$ outage capacity is $19 \%$ over MMSE detection and the respective gain on $1 \%$ outage capacity is $18 \%$. Similarly, Figure 5.7 (b) compares the the AA-PF-MMSE multi algorithm with the AA-PF-VBLAST multi algorithm. For any number of users the AA-PF-VBLAST multi method outclasses the AA-PF-MMSE multi method. Specifically, for in the case of 1 user, there is a gain on average capacity of about $26 \%$ due to VBLAST detection over MMSE detection. For 20 simultaneous users, there is a similar gain on average capacity of about 20\% due to VBLAST detection over MMSE detection. In terms of outage capacities, the gain, for a system with 20 users due to VBLAST detection on $10 \%$ outage capacity is $24 \%$ over MMSE detection and the respective gain on $1 \%$ outage capacity is $25 \%$. These results show that the employment of VBLAST detectors improves the system performance in terms of system capacity and outage capacity both in the case of "single" and "multi" scheduling algorithms. Here, we must point out that in the case of AA-PF multi schemes, the additional improvement due to VBLAST detection tends to decrease when the number of active users is increased. In particular going from 1 user to 20 users, the gain is reduced from $26 \%$ to $20 \%$. The reason for this is that in the case of "multi" schemes, the feasibility constraint imposed by the VBLAST technique restricts the efficiency of the VBLAST based "multi" schemes.

\section{II. "single" schemes vs "multi" schemes}

Figures 5.8(a) and (b) show direct comparisons of the performance of the "single" schemes versus the performance of the "multi" schemes in terms of system capacity and outage capacity. Specifically, Figure 5.8(a) compares the AA-PF-MMSE single scheme with the AA-PF-MMSE multi scheme. For any number of users, the AA-PF-MMSE multi scheme outperforms significantly the AA-PF-MMSE single scheme. In terms of average system capacity, the "multi" scheme provides a gain of $14 \%$ over the respective "single" scheme, for a system with 20 users. For the same number of users, the gain due to "multi" scheme for the $10 \%$ outage capacity is $54 \%$ over the "single" scheme. In terms of $1 \%$ outage capacity, the AA-PF-MMSE multi scheme offers 2.5 times higher capacity than the respective single scheme. The performance of the AA-PF-VBLAST single scheme and the AA-PF-VBLAST multi scheme, is shown in Figure 5.8(b). As in the case of the AA-PF-MMSE schemes (where the "multi" scheme outperforms the "single" scheme), the AA-PF-VBLAST schemes manifest a similar behavior. For any number of users, the AA-PF-VBLAST multi scheme outperforms the AA-PF-VBLAST single 
scheme. In terms of average system capacities, the "multi" scheme is offering a $30 \%$ gain over the respective "single" scheme, for a system with 20 simultaneous users. The gain due to the "multi" scheme for the $10 \%$ outage capacity is $61 \%$ over the "single" scheme and the respective gain on $1 \%$ outage capacity is $420 \%$. Here, note that the AA-PF-VBLAST multi scheme achieves a higher gain over the AA-PF-VBLAST single compared to the respective gain due to AA-PF-MMSE multi scheme over the AA-PF-MMSE single. The VBLAST architecture is proven to exploit multiuser diversity better than MMSE detection.

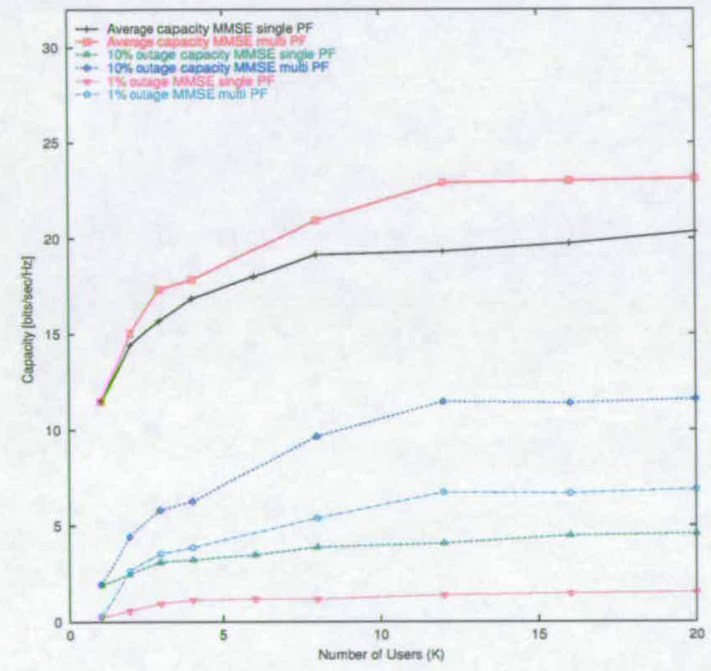

(a)

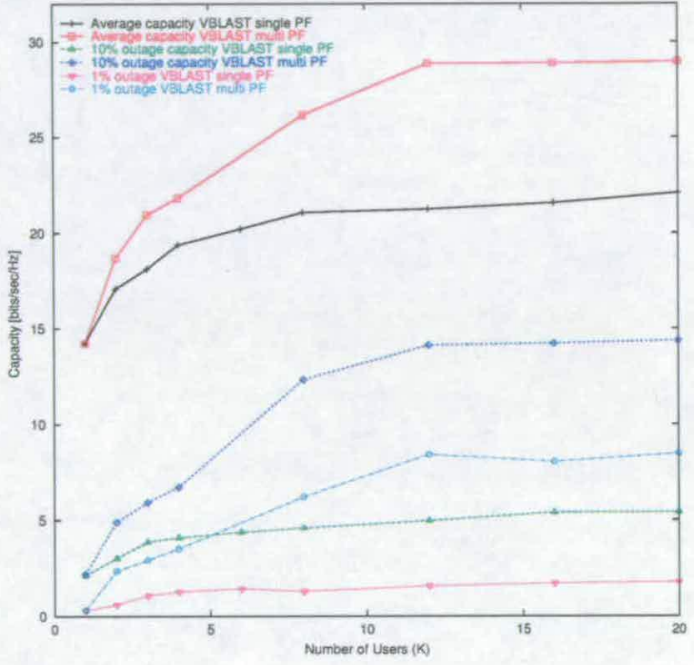

(b)

Figure 5.8: System capacities on a (4,4) MIMO system (SNR at boundary = OdB): (a) AA-PF-MMSE single vs AA-PF-MMSE multi, (b) AA-PF-VBLAST single vs AA-PF-VBLAST multi

\section{AA-PF-VBLAST multi vs AA-PF-VBLAST multi fair}

The AA-PF-VBLAST multi fair scheme, by not allowing multiple antennas to be allocated to the same user, offers more fairness among users, but what happens with the system throughput and the user throughput? Figure 5.9 presents the performance of the AA-PF-VBLAST multi scheme and the AA-PF-VBLAST multi fair scheme in terms of system capacities for a $(4,4)$ MIMO system. The transmit power is fixed to give $0 \mathrm{~dB}$ at the cell boundary $\left(S N R_{0}=0 d B\right)$. First of all, note that for less than $N_{T}(=4)$ users both schemes manifest exactly the same performance. This is expected due to the fact that for a system with active users less than the transmit antennas $\left(K<N_{T}\right.$ ), the AA-PF-VBLAST multi fair scheme allows multiple antennas to be allocated to the same users. For any number of active users more than $N_{T}$, the 


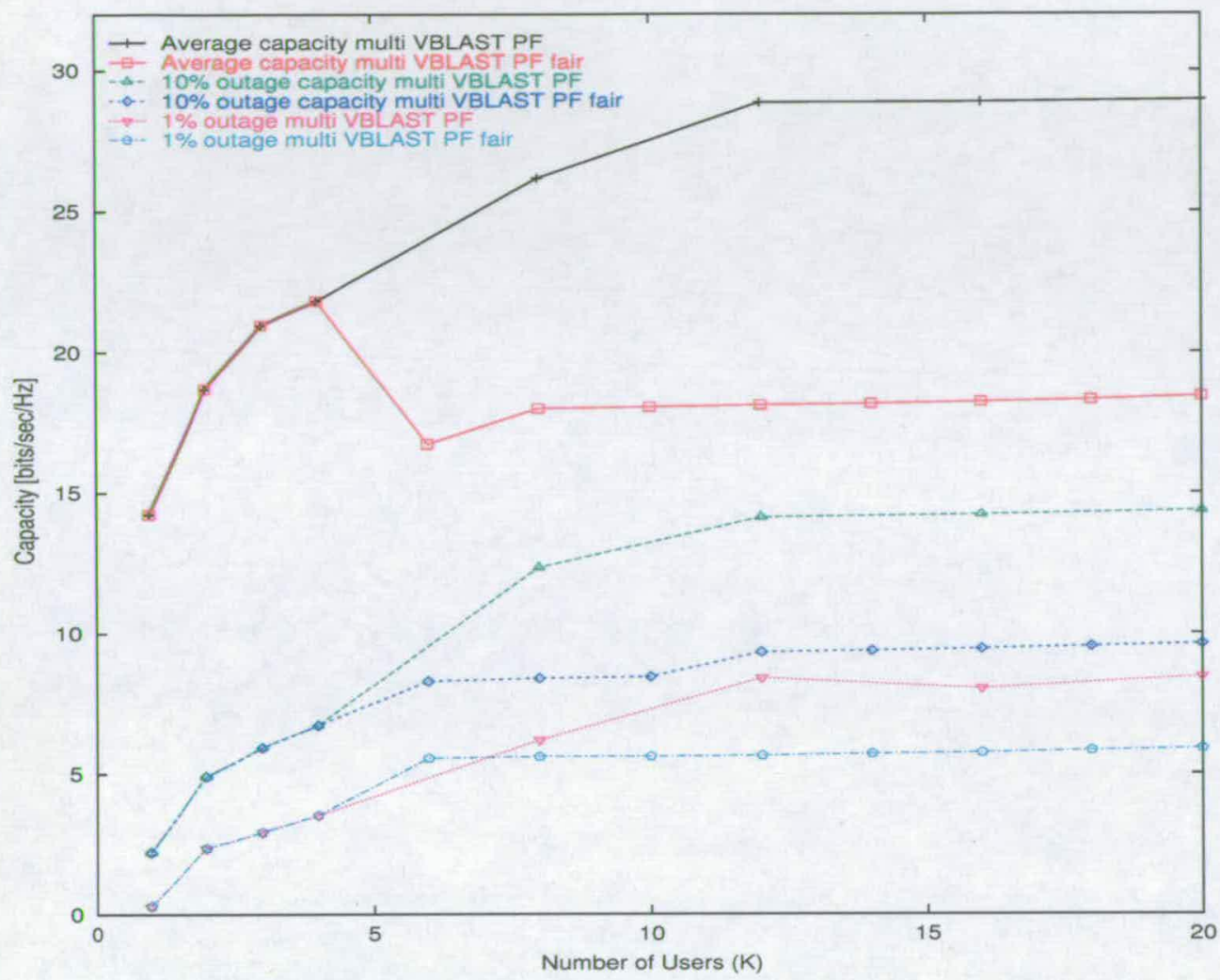

Figure 5.9: System capacities on a $(4,4)$ MIMO system ( $S N R$ at boundary $=0 d B$ )

fairness constraint imposed by the AA-PF-VBLAST multi fair scheme, leads to a degradation of the system performance. Specifically, for 20 users, there is a $58 \%$ decrease in terms of the system capacity due to the AA-PF-VBLAST multi fair scheme. Similar degree of degradation is observed for the outage capacities. The explanation for the degradation in cell throughput due to AA-PF-VBLAST multi fair scheme, is that for any time instant $t$ there would not be in practice more than two users experiencing good channel conditions at the same time (as we can see from Figure 5.11). Which means that in a $(4,4)$ MIMO system the "fair" scheduler will serve two users with good channel conditions and two others with much poorer channel conditions. This assignment will be at the expense of the system throughput.

Figures 5.10(a) and (b) show the cumulative distribution functions of the date rates of different PF scheduling schemes in a $(4,4)$ MIMO system with 20 simultaneous users. There are two types of data rates presented in the graphs, the data rates of "all" users and the data rates of the "selected" users. The data rates of "all" users, are all the requested data rates (per user/per 


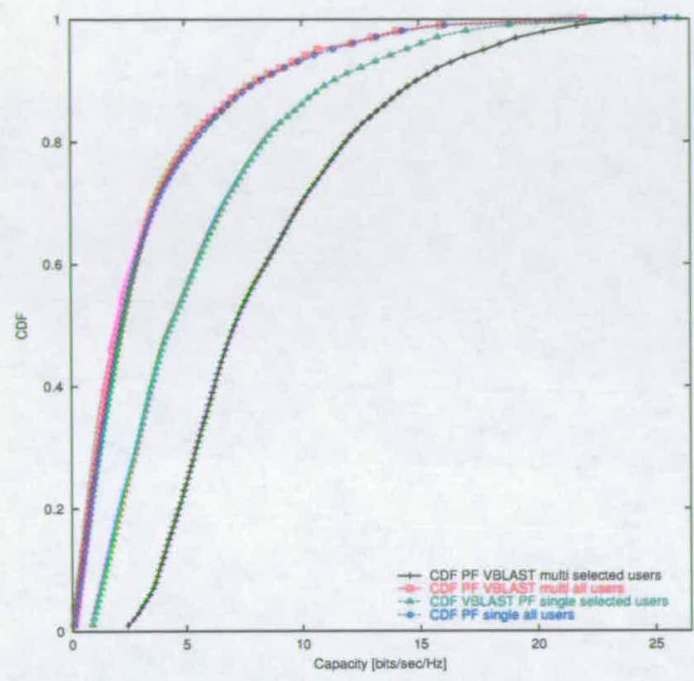

(a)

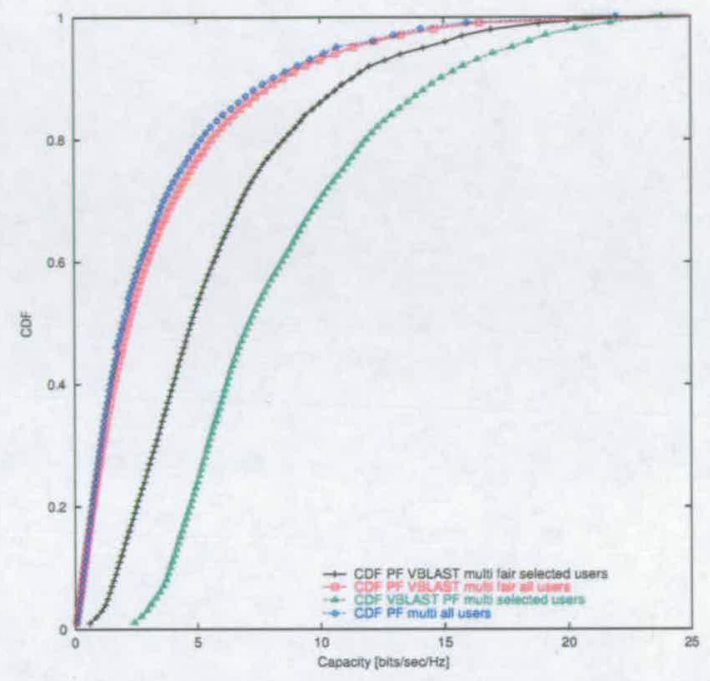

(b)

Figure 5.10: Data Rate per user for a $(4,4)$ MIMO system (SNR at boundary $=$ OdB), with 20 active users: (a) AA-PF-VBLAST single vs AA-PF-VBLAST multi, (b) AA-PF-VBLAST multi vs $A A-P F-V B L A S T$ multi fair

transmit antenna) before the selection of the best users in the proportional fair sense by the scheduler (note that these data rates values are calculated using MMSE detection). The data rates of the "selected" users are the the data rates (per user/per transmit antenna) after the selection of the best users in the proportional fair sense by the scheduler (note that these data rates values are calculated using VBLAST detection). Figure 5.10(a) shows the data rates of the AA-PF-VBLAST multi scheme and the AA-PF-VBLAST single scheme. The graph shows that both schemes have almost the same distributions for "all" calculated data rates as might be expected. On the other hand, there is an increase in the "selected" users data rates due to the AA-PF-VBLAST multi scheme. This implies that there is a multiuser diversity gain, as we would expect due to the AA-PF-VBLAST multi scheme. Note here, that the distribution function of the "selected" data rates are steeper than the cdf of the "all" data rates. This is something we expect because the "selected" data rates are preferentially selected out of the pool of "all" data rates. In Figure 5.10(b), the cumulative distribution functions of the data rates of the AA-PF-VBLAST multi and AA-PF-VBLAST multi fair scheduling schemes are plotted. The system has 20 active users. The graph shows a decrease in the "selected" data rates due to the AA-PF-VBLAST multi fair scheme. The reason for that is that the AA-PFVBLAST multi fair scheduler, by not allowing multiple antennas to be assigned to the same 
user, reduces the set of data rates available for selection.

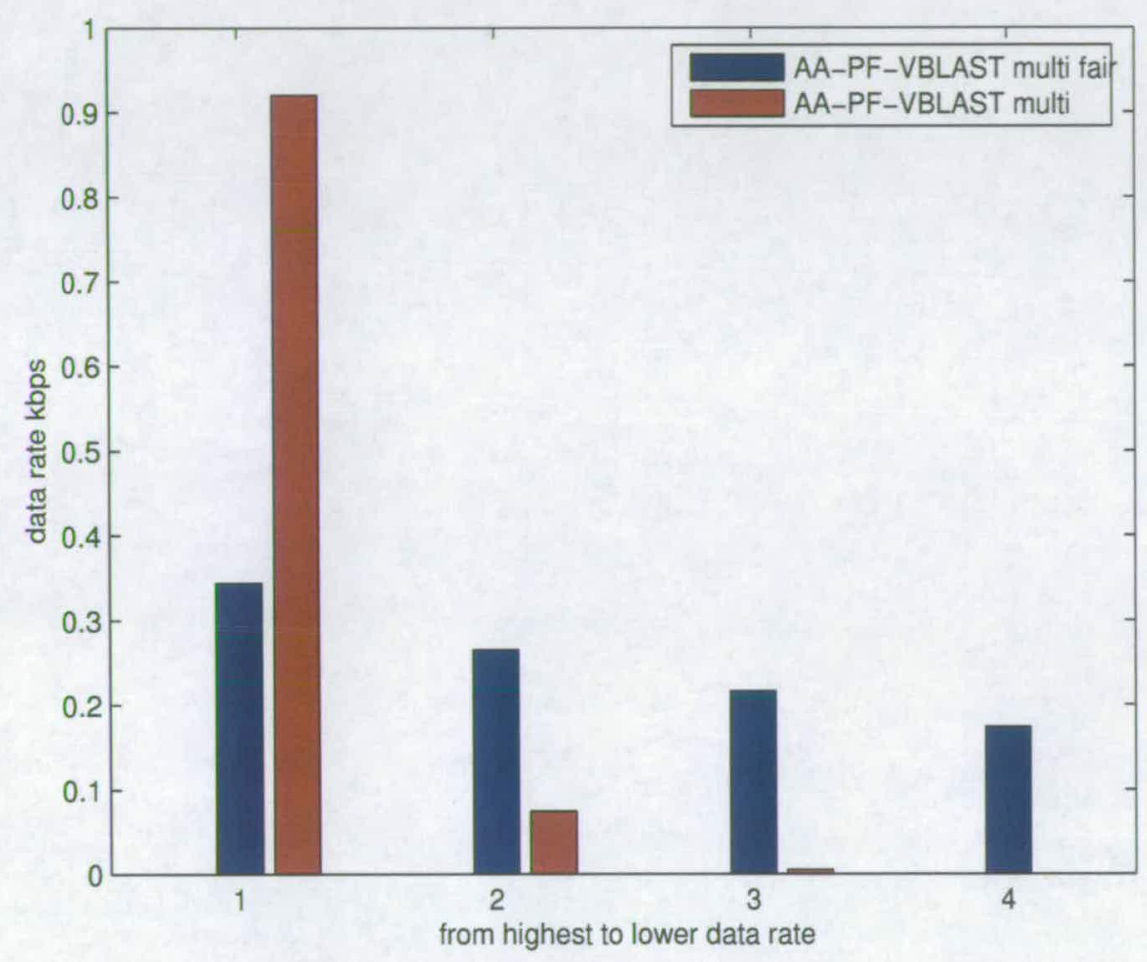

Figure 5.11: Average data rates per scheduled user every time slot for a $(4,4)$ MIMO system, SNR at the boundary equals $O d B, 20$ active users.

In Figure 5.11 we investigate the "outcome" fairness of the AA-PF-VBLAST multi scheme and AA-PF-VBLAST multi fair scheme. This graph depicts the average data rates of each (different) user in the SUG of a $(4,4)$ MIMO system, with 20 simultaneous users, normalized by the system capacity. The purpose of this graph is to show how the system capacity available in each time slot is shared by the users. In the simulated system, no more than 4 users may be served each time slot (there are $N_{T}=4$ transmit antennas). It is shown that the AA-PFVBLAST multi fair scheme provides a higher level of fairness among users. Namely, in each time slot the AA-PF-VBLAST multi fair scheduler allocates the system capacity among $N_{T}$ users (in our system there are $N_{T}=4$ transmit antennas). On the other hand, the AA-PFVBLAST multi scheduler in practice serves only 2 users at each time slot, allocating $92 \%$ of the system capacity to the first user and the other $8 \%$ to the second. Note here, that the plotted 
values are normalized to system capacity. The absolute values of the system capacity for these scheduling schemes are plotted in the Figure 5.9.

\section{AA-PF-VBLAST multi vs VBLAST AA-RRS and AA VBLAST Fair}

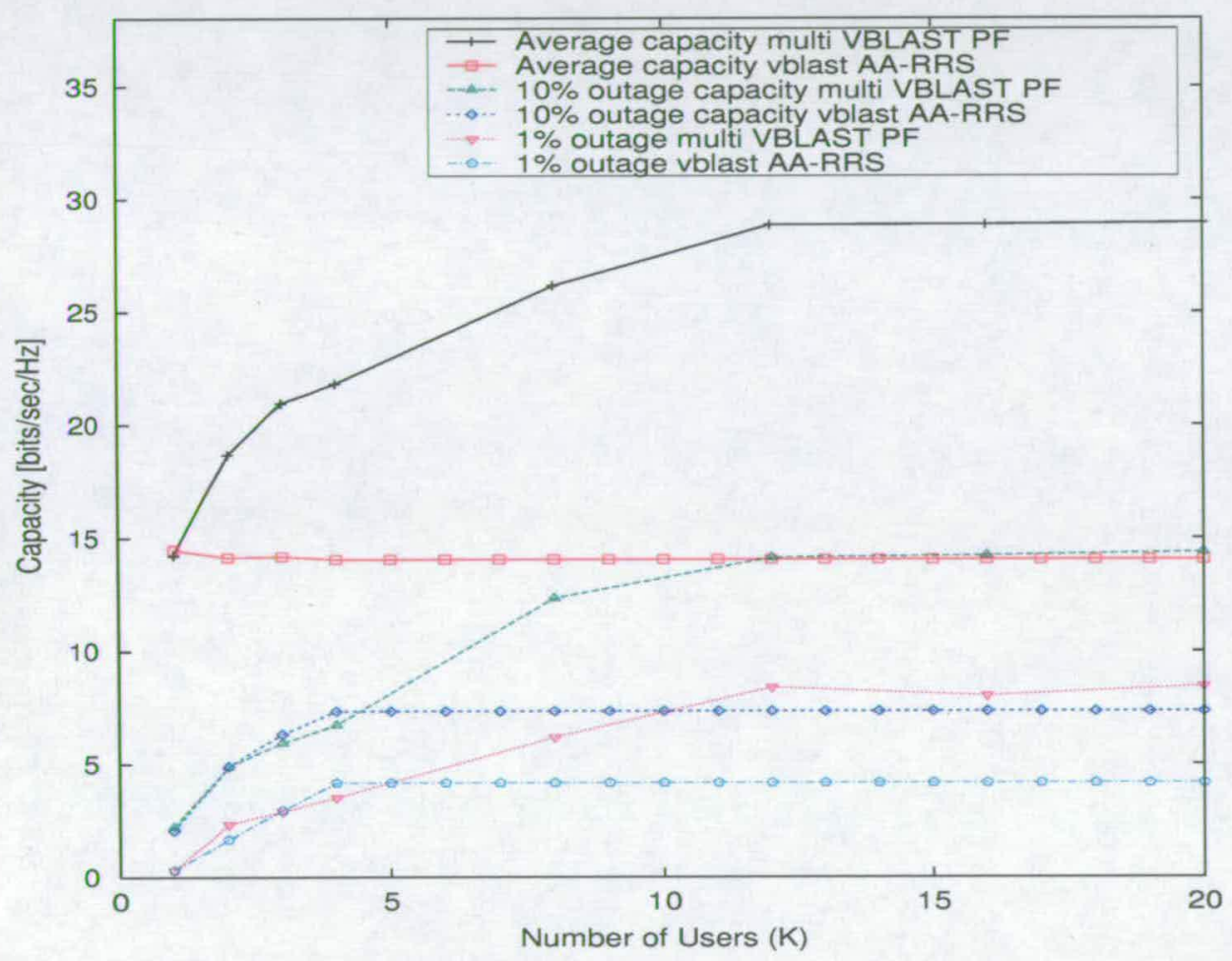

Figure 5.12: System capacities on a $(4,4)$ MIMO system $(S N R$ at boundary $=0 d B)$

In this section we compare the AA-PF-VBLAST multi scheme with two scheduling schemes presented in Chapter 4. Specifically we compare AA-PF-VBLAST multi scheme with the VBLAST AA-RRS and the AA VBLAST Fair schemes. Figure 5.12 presents the performance of the AA-PF-VBLAST multi scheme and the VBLAST AA-RRS scheme (section 4.4.1) in terms of system capacities for a $(4,4)$ MIMO system. The transmit power is fixed to give 0 $\mathrm{dB}$ at the cell boundary $\left(S N R_{0}=0 d B\right)$. It is obvious that there is a significant gain due to AA-PF-VBLAST multi scheme. Analytically, the system capacity for AA-PF-VBLAST multi schemes increases with the number of users $k$ giving a $106 \%$ gain on system capacity over the VBLAST AA-RRS (doubles the capacity) in case of 20 simultaneous users. For outage capacity $10 \%$ there is $91 \%$ gain and for $1 \%$ outage capacity there is $84 \%$ gain. This is because the AA-PF-VBLAST multi scheme has a larger user selection range than the VBLAST AA- 
RRS scheme. In VBLAST AA-RRS scheme only $N_{T}$ predefined users by the RRS algorithm are available for selection each time slot. On the other hand, in AA-PF-VBLAST multi scheme the scheduler can chose any of the $k$ simultaneous users. This feature of the AA-PF-VBLAST multi scheme leads to a stronger diversity effect and higher system capacity.

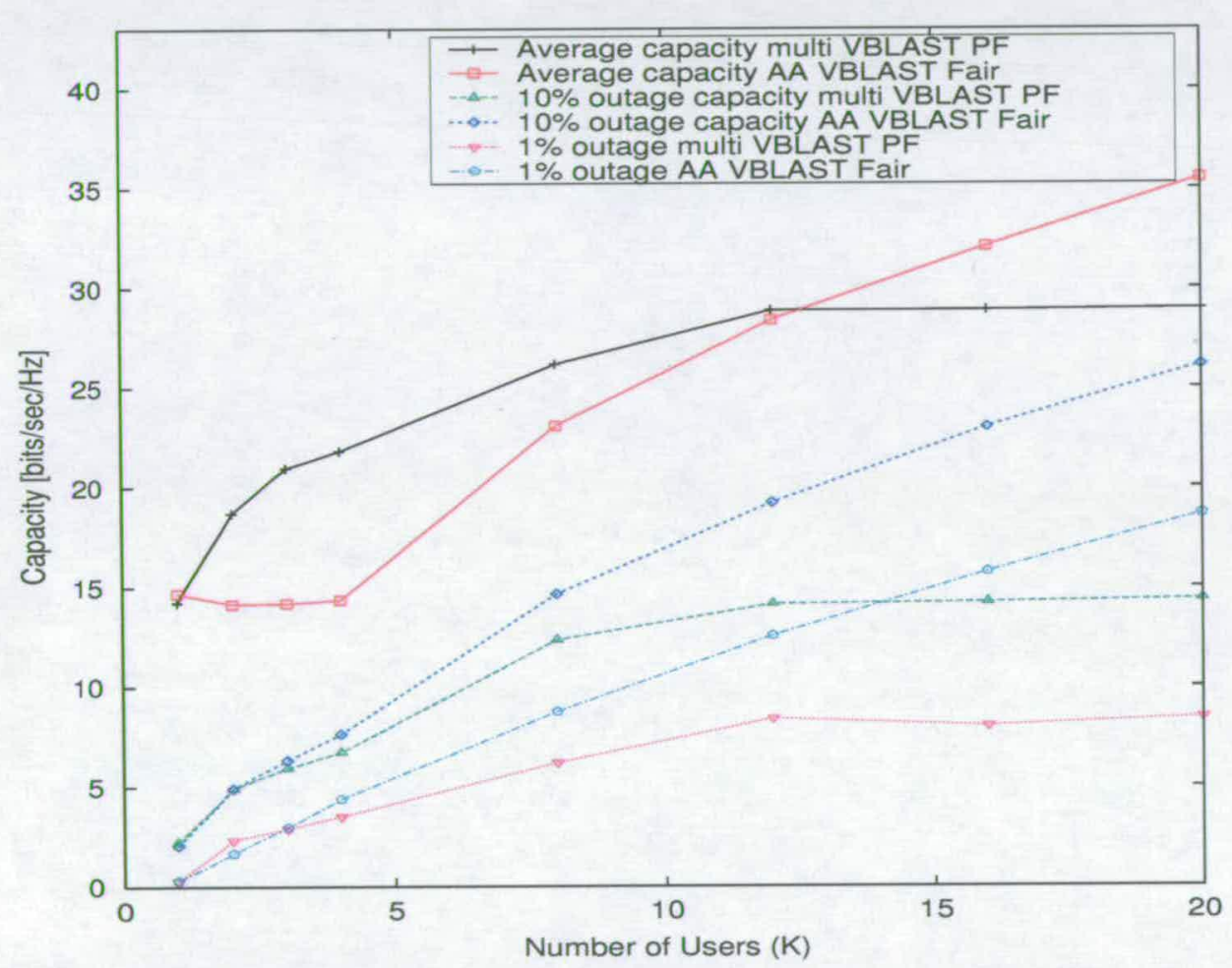

Figure 5.13: System capacities on a $(4,4) M I M O$ system ( $S N R$ at boundary $=0 d B$ )

Figure 5.13 compares the performance of the AA-PF-VBLAST multi scheme with the AA VBLAST Fair scheme (section 4.4.5) in terms of system capacities for a $(4,4)$ MIMO system. The transmit power is fixed to give $0 \mathrm{~dB}$ at the cell boundary $\left(S N R_{0}=0 d B\right)$. The graph shows that the AA VBLAST Fair scheme provides a higher system capacity than the AA-PFVBLAST multi scheme for more than 12 active users. On the other hand for less active users the AA-PF-VBLAST multi scheme offers a average capacity gain. In terms of outage capacity, the AA VBLAST Fair scheme outperforms the AA-PF-VBLAST multi scheme for the whole range of active users, but again for more than 12 users this improvement due to AA VBLAST Fair scheme is more clear. 


\subsubsection{Number of Transmit and Receive Antennas}

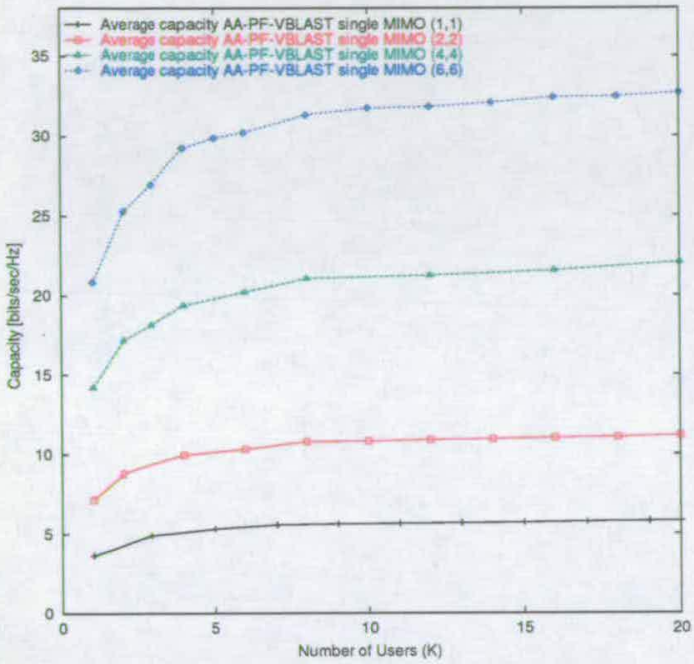

(a)

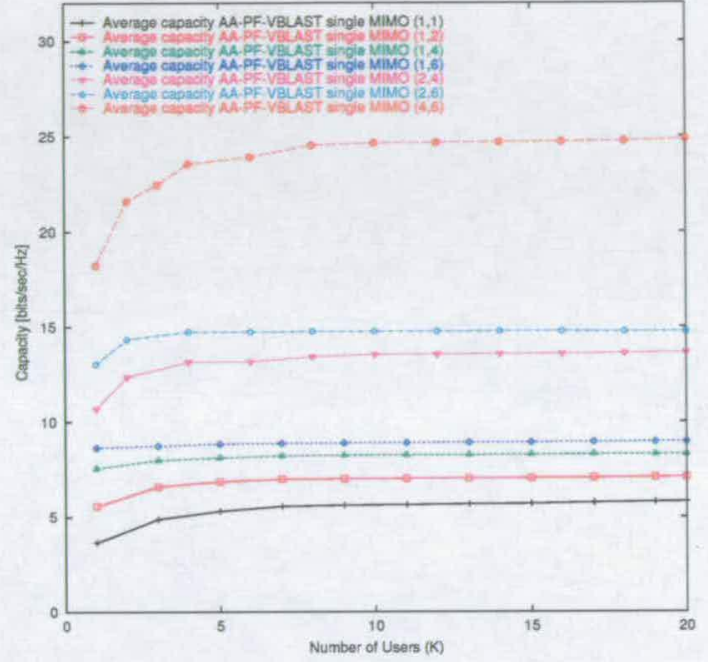

(b)

Figure 5.14: System capacities on a $(4,4)$ MIMO system (SNR at boundary =OdB) - AA-PF-VBLAST single scheme: (a) symmetrical MIMO arrays (b) asymmetrical MIMO arrays

In this section, we investigate the antenna diversity effect of MIMO systems with different numbers of transmit and receive antennas for different PF scheduling schemes. In the simulations carried out in this section the transmit power is fixed to give $0 \mathrm{~dB}$ SINR at the cell boundary $\left(S N R_{0}=0 \mathrm{~dB}\right)$. We separate our results to two groups. The first group is for symmetrical MIMO arrays, where the number of antenna arrays at the transmitter is equal to the number of the antenna arrays at the receiver. This group consists of the following MIMO systems: $(1,1)$, $(2,2),(4,4)$ and $(6,6)$. The second group is the asymmetric MIMO arrays, where there are more antenna arrays at the receiver than at the transmitter. This group consists of the MIMO systems: $(1,2),(1,4),(1,6),(2,4),(2,6)$ and $(4,6)$. In Figure 5.14(a) and (b) we compare the system capacities values of the symmetrical MIMO arrays and asymmetrical MIMO arrays respectively, in case of AA-PF-VBLAST single scheduling, with respect to the number of simultaneous users. In case of transmit diversity, namely when the number of transmit antennas is increasing we have the following results (for the case of 20 simultaneous users). Going from $(1,2)$ to $(2,2)$ results in a $57 \%$ increase in system average capacity. Going from $(1,4)$ to $(2,4)$ the increase is $64 \%$ and going from $(1,4)$ to $(4,4)$ produces an impressive increase of $166 \%$. The above results point out that increased spatial multiplexing provides a significant capacity benefit on 
the AA-PF-VBLAST single scheme.

In case of receive diversity we have the following results for a system with 20 active users. Going from $(2,2)$ to $(2,4)$ results in a $22 \%$ increase in system average capacity. Going from $(4,4)$ to $(4,6)$ the increase is $12 \%$ and going from $(2,2)$ to $(2,6)$ we notice an increase of $32 \%$. These results show that the AA-PF-VBLAST single is not as efficient with receive diversity as in the case of transmit diversity. In case of combined receive and transmit diversity we have the following results for 20 active users. Going from $(2,2)$ to $(4,4)$ results in a $98 \%$ increase in system average capacity (doubling the number of antennas results in double system capacity). Going from $(2,2)$ to $(6,6)$ the increase is $193 \%$.

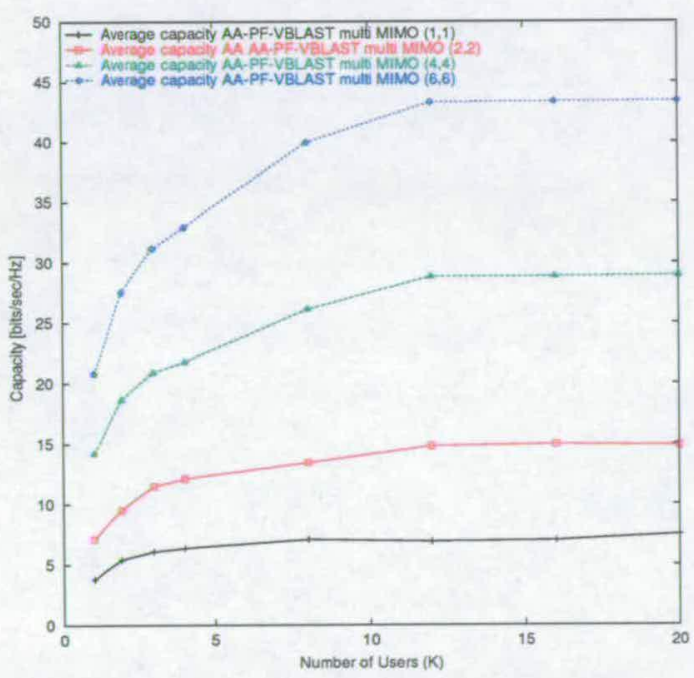

(a)

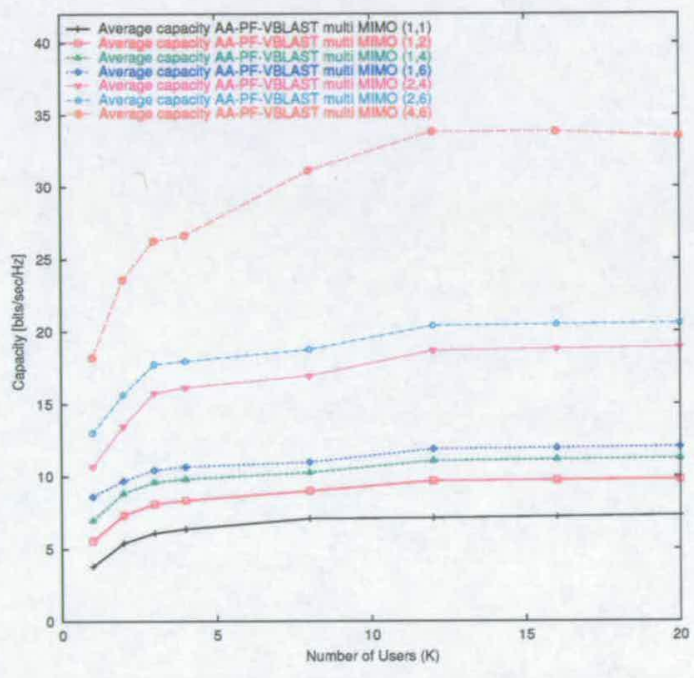

(b)

Figure 5.15: System capacities on a (4,4) MIMO system (SNR at boundary $=0 d B)-A A-P F-V B L A S T$ multi scheme: (a) symmetrical MIMO arrays, (b) asymmetrical MIMO arrays

In Figure 5.15(a) and (b) we compare the system capacities values of the symmetrical MIMO arrays and asymmetrical MIMO arrays respectively, in case of AA-PF-VBLAST multi scheduling, with respect to the number of simultaneous users. Analyzing the simulation results to investigate the antenna arrays diversity effect, we notice that the graphs of system capacities for the AA-PF-VBLAST multi algorithm are similar to the ones studied before in the case of the AA-PF-VBLAST single scheme. Note particularly that the transmit diversity is the most efficient antenna diversity method for improving the system capacity. On the other hand the gains are not so large in the case of receive diversity and in the case of combined receive and transmit 
diversity.

\subsubsection{Time Delay per user}

In Figure 5.16(a) we investigate the "service" or "effort" fairness (measured in terms of time delay) between the AA-PF-VBLAST single scheme and the AA-PF-VBLAST multi scheme. Time delay is defined as the number of idle time slots that have occurred since the last packet was sent to the same user (the delay concept has been discussed extensively in Section 4.5.1.2). Results for the time delay of the RRS scheme are also available for comparison in the same graph. Note that the RRS scheme serves $N_{T}$ users per time slot, where $N_{T}$ is the number of transmit antennas. The graph presents results for the cases of 8 mobile users and 32 mobile users. The time delay per user between two consecutive transmissions is used as a measure of "effort" faimess. Note, that the round robin scheduler provide the same time delay for all users. On the other hand, the AA-PF-VBLAST single scheme gives a really wide spread of time delays per user, which makes this scheduler less fair than the RRS and AA-PF-VBLAST multi schemes. For any given time delay value, the probability that the AA-PF-VBLAST single scheduler offers a shorter delay per active user than this value, is always smaller than the respective probability for the AA-PF-VBLAST multi scheme. However, this is balanced by AA-PF-VBLAST single scheduler delivering more data per packet. Specifically, for a system with 32 active users, the likelihood of one user experiencing a delay shorter than 7 time slots, equals to $16 \%$ for the AA-PF-VBLAST single scheme. The corresponding likelihood, for the case of AA-PF-VBLAST multi scheme is $28 \%$.

Figure 5.16(b) compares the distribution of the time delays of the active users for the case of the AA-PF-VBLAST multi scheme and the case of the AA-PF-VBLAST multi fair scheme. The graph shows that the AA-PF-VBLAST multi fair scheme is even more fair than the AAPF-VBLAST multi scheme. Specifically, for system with 32 active users, the possibility that one user will be served every 7 time slots or less is $28 \%$ in the case of the AA-PF-VBLAST multi scheme and $68 \%$ in the case of the AA-PF-VBLAST fair scheduler. Clearly, the AAPF-VBLAST multi fair scheduler guarantees a shorter waiting time between consecutive transmissions for any user than the AA-PF-VBLAST multi scheme or the AA-PF-VBLAST single scheme. However, this comes at cost of reducing cell throughput as shown in Figure 5.9. 


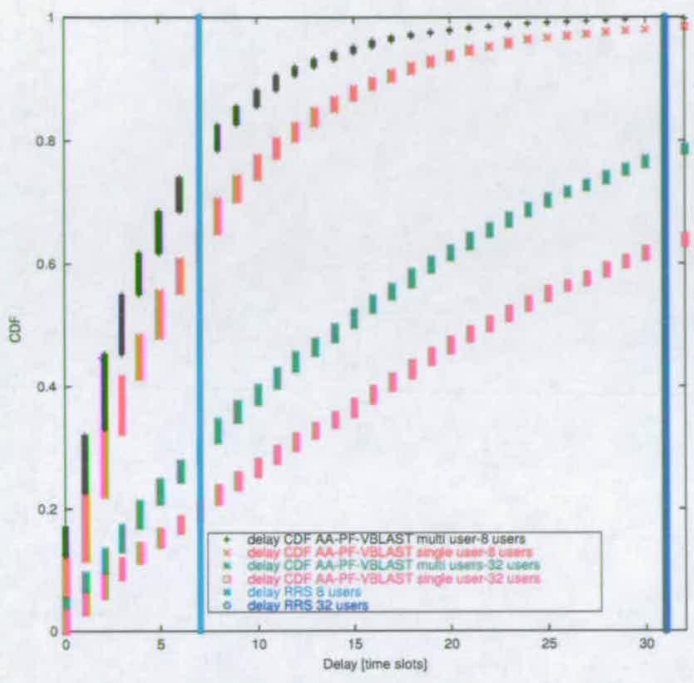

(a)

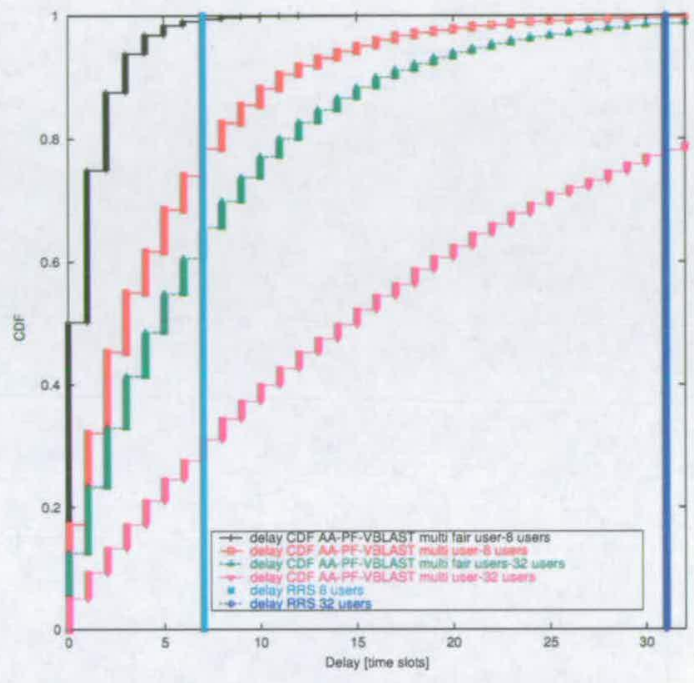

(b)

Figure 5.16: Time delay per scheduled user for a $(4,4)$ MIMO system (SNR at boundary = OdB): (a) $A A-P F-V B L A S T$ single vs AA-PF-VBLAST multi, (b) AA-PF-VBLAST multi vs AA-PFVBLAST multi fair

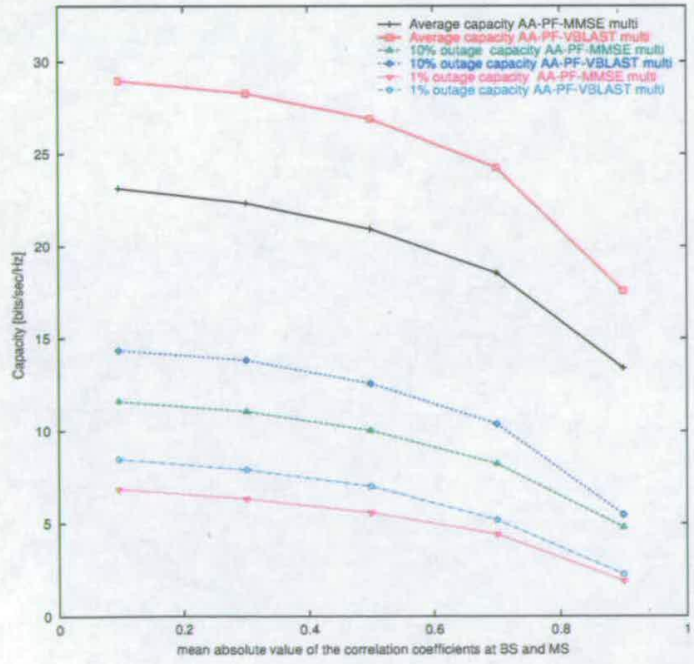

(a)

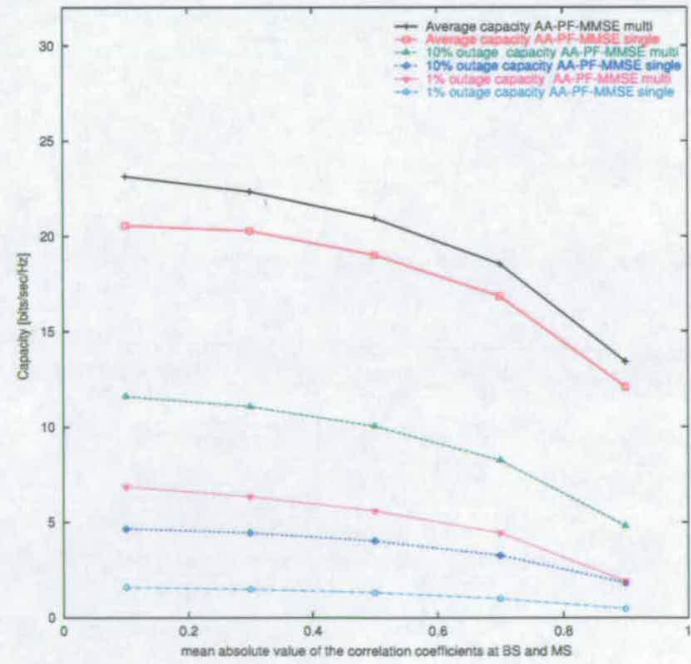

(b)

Figure 5.17: Effect of spatial correlation on a (4,4) MIMO system with 20 active users (SNR at boundary $=O d B$ ), the correlation (at both the BS and MS) takes values from 0.1 to 0.9: (a) AA-PF-MMSE multi vs AA-PF-VBLAST multi, (b) AA-PF-MMSE multi vs AA-PF-MMSE single 


\subsubsection{Spatial Fading Complex Correlation}

The effect of spatial fading complex correlation on different PF scheduling schemes is investigated in this section. Many different correlation scenarios are investigated. The basic idea is to start from a decorrelated scenario, where both the Base Station (BS) and the Mobile Station (MS) are decorrelated (the mean absolute value of the coefficients is 0.1 ). Gradually, we increase the correlation at both the base station and the mobile station. The correlation is increasing from 0.1 to 0.9 , and the results for the system capacities are presented. The method for generating the correlated channel matrices is explained in Chapter 3. In Figure 5.17(a) we measure the performance of the AA-PF-MMSE multi scheme and the AA-PF-VBLAST multi scheme, in terms of system capacities for a system with 20 simultaneous users. The transmit power is fixed to give $S N R_{0}=0 d B$. The AA-PF-VBLAST multi scheme provides a better performance than the AA-PF-MMSE multi scheme for any degree of correlation. Specifically, for decorrelated channel, where the mean absolute value of the coefficients is 0.1 at both the BS and the MS (Figure 5.17), there is a $21 \%$ gain due to AA-PF-VBLAST multi on average capacity. Also, there are similar gains in outage capacities. For outage capacity $10 \%$ the gain is $24 \%$ and for outage capacity $1 \%$ the gain is $23 \%$. If we compare these results with the results of high correlated channel (correlation 0.9 at both ends) we will see that the AA-PF-VBLAST multi scheme is more efficient than AA-PF-MMSE multi scheme in terms of average system capacity. Specifically, the gain due to AA-PF-VBLAST on average capacity is $28 \%$ (7\% more gain than in case of decorrelated channel), and the gains on outage capacities $10 \%$ and $1 \%$ are $20 \%$ and $22 \%$ respectively.

Figure 5.17(b) investigates the effect of spatial fading correlation on the multiuser diversity. The system capacities and outage capacities of $(4,4)$ MIMO system are evaluated and plotted in the same graph. According to the results, it is clear that the AA-PF-MMSE multi scheme performs better than the AA-PF-MMSE single scheme for any degree of correlation.

\subsection{Conclusions}

In this chapter, we considered the problem of designing scheduling algorithms based on PF scheme for multiuser MIMO systems with low complexity. Sophisticated use of diversity in all its forms is the key tool that is the focus of this chapter. The intricate interplay of different forms of diversity is an important concept for reliable wireless networks.

The goal of this chapter is to apply spatial multiplexing techniques in order to achieve multiuser 
diversity. Multiuser diversity arises from independent fading channels across different users. We investigated the performance of a different PF scheduling schemes in terms of system capacity, fairness and time delay. We have simulated the following schemes: AA-PF-MMSE single, AA-PF-MMSE-multi, AA-PF-VBLAST single, AA-PF-VBLAST multi and AA-PF-VBLAST multi fair. The most important conclusions from the study of these scheduling schemes are summarized in the following points:

- The AA-PF-VBLAST multi scheme offers the highest average and outage cell capacities.

- The AA-PF-VBLAST multi fair scheme offers minimum packet-to-packet delay among the simultaneous users, but at the expense of throughput, compared to the single scheme.

- The AA-PF-VBLAST single (multi) scheme outperforms the AA-PF-MMSE single (multi) scheme in terms of system capacity and outage capacity for any communication scenario (e.g. for any number of simultaneous users or for any degree of spatial fading correlation). At the same time AA-PF-VBLAST single (multi) scheme shows the same degree of faimess as the AA-PF-MMSE single (multi) scheme by definition, as long as both schemes use the same proportional fairness criterion (equation (5.3)).

- The AA-PF-MMSE multi scheme outperforms the AA-PF-MMSE single scheme in terms of system capacity and outage capacities. Note, that the multiuser diversity is proven to be beneficial for the system performance in terms of system throughput.

- The AA-PF-VBLAST multi scheme provides better cell throughput than the AA-PFVBLAST single scheme. Specifically, for a system where the mobile users are more than $\min (M, N)$, the gain because of the AA-PF-VBLAST multi scheme is reaching its peak.

- There is a trade-off between system capacity and time delay. Generally, those schemes that display the highest average system capacity also show the highest average delays per user.

- In conclusion AA-PF-VBLAST multi scheme outperforms any other scheduling scheme in terms of system capacity without affecting the fairness among users.

There are a variety of fruitful areas for future research on proportional fair packet scheduling, multiuser diversity and related topics. The results from this chapter suggest that the use of VBLAST detectors does enhance the performance of the proportional fair schemes, although. 
the feasibility constraints implied by the VBLAST detection tends to counteract the synergy of multiuser diversity and spatial multiplexing. On the other hand, the use of multiuser diversity improves significantly the system performance in terms of system capacity, and at the same time guarantees a higher degree of fairness among the simultaneous users.

\subsubsection{Future Work}

Real time applications may have stringent packet delay constraints which would change the evaluation and alter the presented results. It was also assumed that the transmission rate (capacity) instantaneously could adapt to the optimum value through the relationship between achievable capacity and the instantaneous received SINR. Future work may include more accurate link adaptation models as well as dynamic range limitations. Finally, considering both uplink and downlink scenarios, we need to create a more complete picture of the cellular network and introduce encryption for security reasons (each user may need to decode data for another user). 


\section{Chapter 6 Summary - Conclusions}

In this work, a number of existing signal processing techniques were examined. The aim of this investigation was to explore the efficiency of these methods in a third or fourth generation wireless system where the demands for higher data rates and QoS is ever increasing. For this purpose, we compared a number of technologies (such as MIMO arrays, VBLAST detection and RRS or PF scheduling schemes) in various scenarios of interest, in terms of system efficiency (cell capacity) and QoS metrics. The comparison has provided as with an indication about the performances of these methods and led us to propose some new algorithms that aim to combine the benefits from previously examined wireless techniques. This final chapter summarizes in Section 6.1 the main topics and conclusions contained within this thesis. Section 6.2 presents the limitations of this work and proposes areas that this work can be extended to consider.

\subsection{Summary and thesis contributions}

The presented work can be broken down into four logical parts: the first part (Chapter 2) provides a survey of the recent developments in the field of wireless communication, focusing on some specific concepts such as multiple-input multiple-output (MIMO) antennas and packet scheduling; the second part (Chapter 3) concentrates on the downlink physical channel level of the communication and selects the downlink MIMO channel model that will be used throughout this work; the third part (Chapter 4) presents the performance of existing packet scheduling schemes based on RRS scheme and proposes some new scheduling schemes exploiting the VBLAST architecture, and finally the fourth part (Chapter 5) examines the performance of the PF scheduling scheme and proposes some new schemes based on PF scheme and VBLAST algorithm. We now summarize of the main conclusion of each part.

In Chapter 2 an initial study of a wireless communication system and its basic components is presented. Special attention has been paid to the technologies of interest in this work, such as MIMO systems and packet scheduling strategies. According to the theoretical results (found 
in the literature) there are great potential capacity gains which can be realized from MIMO architectures. In particular, the system capacity (equation (2.5)) of a MIMO system with $N_{T}$ transmitters and $N_{R}$ receivers grows linearly with $m=\min \left(N_{T}, N_{R}\right)$. Note here, that whether we achieve this fully or at least partially in practice depends on a sensible design of transmit and receive signal processing algorithms. Regarding the study of packet scheduling policies Chapter 2 concludes that there are tradeoffs among the throughput, delay, and fairness of an scheduling algorithm. Generally, those schemes that display the highest average sector throughputs also show the highest average delays per user, and the largest variations in the amount of resources allocated to each user. Schemes that display lower throughputs also provide lower average delays per user, and a fairer distribution of resources. These background results led us in constructing efficient scheduling schemes in the next chapters (Chapters 4 and 5).

Next, in Chapter 3 we investigate the downlink MIMO physical channel performance. We split down the system model into three main parts (transmitter, physical channel and receiver) and analyze each part separately. After a survey of the state of the art in MIMO channel models, we select the channel model that is used throughout this work. The main purpose of this chapter was to describe and investigate the structure of our MIMO physical channel model and characterize the performance of this channel. Detection algorithms (MMSE and VBLAST MMSE) for single-user wireless communication using multiple antennas at both the transmitter and receiver in a stochastic MIMO radio channel model were compared for different degrees of correlation between the antenna array elements. The effect of error propagation was also investigated. All the simulation scenarios have been implemented for two modulation schemes (16-QAM and BPSK). The stochastic MIMO channel model was simulated using both a measured and theoretical fading correlation model. With reference to the BER simulation results of this chapter it is obvious that the spatial fading complex correlation between antenna arrays leads to a significant degradation of the system performance.

In the next part of this thesis, our goal is to exploit multiuser diversity of a MIMO system using VBLAST architecture and Round Robin (RRS) scheduling techniques. The cooperation of VBLAST detection and RRS packet scheduling in a MIMO system creates data rate feasibility constraints imposed by the VBLAST technique. Then, Chapter 4 studies a number of proposed packet scheduling schemes based on existing round robin schedulers that are applied in a $\left(N_{T}, N_{R}\right)$ MIMO multiuser system. In particular, we propose three different packet scheduling algorithms: (I) best user algorithm, implemented in AA-RRS best user scheme and VBLAST AA-RRS best user scheme, (II) VBLAST detection, implemented in VBLAST AA- 
RRS scheme, VBLAST AA-RRS best user scheme and AA VBLAST fair scheme, (III) AA (antenna assisted) fair algorithm, implemented in AA MMSE fair scheme and AA VBLAST fair scheme. Computer simulations have been conducted to compare these scheduling schemes. The results have shown that the employment of VBLAST detectors at the receivers has proven to be beneficial for the system performance, especially in the case of correlated channel or single user systems. The VBLAST RRS scheme gives the highest average system capacity, but the VBLAST AA-RRS scheme is more efficient for delay sensitive systems (better outage capacity results). Also, the best user algorithm is proven to give better efficiency results (higher system throughput) but at the expense of faimess. Finally, the antenna assisted (AA) fair algorithm (AA MMSE fair and AA VBLAST fair) improves the system efficiency in terms of overall capacity and at the same time provides the same level of fairness among users for the time period of $\left\lceil\frac{K}{N_{T}}\right\rceil$ time slots, where $K$ is the number of simultaneous users and $N_{T}$ the number of transmit antennas. Two points are worth mentioning here. First, each user $k$ may need to decode data for another user, which may require the use of encryption for security reasons. Second, we do not consider error-propagation effects on VBLAST performance.

In the last part of this work (Chapter 5), we considered the design of scheduling algorithms based on the PF scheme for multiuser MIMO systems. Intelligent use of diversity in all its forms (multiuser or spatial diversity) is the key tool that was the focus in this chapter. The intricate interplay of different forms of diversity is an important concept for reliable wireless networks, so that high system throughput and QoS requirements could be met at the same time. The goal of this chapter is to combine spatial multiplexing techniques (MIMO and VBLAST) with PF scheduling in order to enhance multiuser diversity. Note that multiuser diversity arises from independent fading channels across different users, and such a form of diversity comes for free in a fading channel. The following PF schemes have been examined: AA-PF-MMSE single, AA-PF-MMSE-multi, AA-PF-VBLAST single, AA-PF-VBLAST multi and AA-PFVBLAST multi fair. The most important conclusions from the study of these schemes are summarized in the following points: (I) the AA-PF-VBLAST multi scheme offers the highest average and outage cell capacities, (II) the AA-PF-VBLAST multi fair scheme offers the minimum packet-to-packet delay among the simultaneous users, but at the expense of throughput, compared to the single scheme, (III) the AA-PF-VBLAST single (multi) scheme outperforms the AA-PF-MMSE single (multi) scheme in terms of system capacity and outage capacity for any wireless scenario (e.g. for any number of simultaneous users or for any degree of spatial fading correlation), (IV) the AA-PF-MMSE multi scheme outperforms the AA-PF-MMSE sin- 
gle scheme in terms of system capacity and outage capacities, and (V) AA-PF-VBLAST multi scheme provides better cell throughput than the AA-PF-VBLAST single scheme.

The above conclusions indicate the beneficial effect of using VBLAST architecture in a MIMO system. Also, it has been demonstrated that the cooperation of multiple antennas, VBLAST architecture and packet scheduling leads to an improved system in terms of system efficiency (cell throughput) and QoS (faimess among the users and outage capacity).

\subsection{Limitations of the work and scope for further research}

Throughout this thesis we assumed that the transmitter (BS) has a perfect knowledge of the downlink channel. We take the quasi-stationary viewpoint that the channel time variation is negligible over the $L$ symbol periods composing a burst, and that the channel is estimated accurately, e.g. by use of a training sequence embedded in each burst. The use of error-correction coding (such as convolutional coding) is assumed. Therefore, we do not consider error propagation effects on VBLAST detection, apart from Chapter 3 where we do investigate its impact on bit error ratio results. In practice, the accuracy of the transmit channel estimation depends on the channel characteristics. Use of reciprocity or feedback methods for channel estimation have been proposed [146]. In each case, estimation errors depend on angle, delay and Doppler spreads. The relative influence of these spreads again depends on duplexing method. Doppler spread induced by the motion of subscribers or scatterers has a strong influence on space-time processing algorithms. The Doppler spread is large in macro-cells which serve high mobility subscribers and it increases with higher operating frequencies. Doppler spread is also present in low mobility (microcell) or fixed wireless networks due to mobility of scatterers. Another point that needs further consideration is the channel coding. Use of coding along with multiple antennas can improve error correction, diversity and co-channel mitigation performance.

Regarding possible extensions to this work, it would be interesting to investigate the proposed techniques towards system implementation so that more realistic figures of data rate values could be available, since the data rates and throughput results in the presented work are based on the theoretical Shannon capacity formula. Also the simulation results could be extended in the case of multi-cell scenarios. 


\section{References}

[1] B. Mahon, "The Man Who Changed Everything : The Life of James Clerk Maxwell," Chichester, West Sussex: John Wiley \& Sons, 2003.

[2] J. G. O'Hara and D. W. Pricha, "Hertz and the Maxwellians: A Study and Documentation of the Discovery of Electromagnetic Wave Radiation, 1873-1894 (Iee History of Technology, 8)," Institution of Electrical Engineers (IEE), 1987.

[3] K.W. Richardson, "UMTS overview," Electronics and Communication Engeneering Journal, pp. 93-101, June 2000.

[4] H. Holma and A. Toskala, "WCDMA for UMTS, Radio Access For Third Generation Mobile Communications," Wiley, Chichester, UK, third edition, 2004.

[5] Nokia Corporation, "Nokia HSDPA Solution," White Paper, www.nokia.com, 2003.

[6] UMTS Forum, "White Paper No 1. Evolution to 3G/UMTS Services," www.umtsforum.org, Aug 2002.

[7] Frodigh M. et al., "Future-Generation Wireless Networks," IEEE Personal Communications, Oct 2001.

[8] M. Tayal, "Location services in the GSM and UMTS networks," Personal Wireless Communications, 2005. ICPWC 2005. 2005 IEEE International Conference on, pp. 373378, Jan 2005.

[9] J. Ling D. Avidor, D. Furman and C. Papadias, "On the financial impact of capacityenhancing technologies to wireless operators," Wireless Communications, IEEE, vol. 10, no. 4, pp. 62-65, Aug 2003.

[10] J. Grewal and J. DeDourek, "Provision of QoS in wireless networks," Communication Networks and Services Research, 2004. Proceedings. Second Annual Conference on, pp. 337-340, May 2004.

[11] R. Lupas and S. Verdu, "Linear multiuser detectors for asynchronous code-division multiple-access channels," IEEE Transactions on Information Theory, vol. 35, no. 1, pp. 123-136, Jan 1989.

[12] P. M. Grant J. S. Thompson, J. E. Hudson and B. Mulgrew, "CDMA downlink beamforming for frequency selective channels," in proceedings of the IEEE International Symposium on Personal, Indoor and Mobile Radio Comunications (PIMRC), pp. 233$237,1999$.

[13] S. Verdu, "Multiuser Detection," Cambridge University Press, USA, 1st edition, Sep 1998. 
[14] J. B. Andersen, "Antenna arrays in mobile communications: Gain diversity, and channel capacity," IEEE Antennas and Propagation Magazine, vol. 42, no. 2, pp. 12-16, Apr 2000 .

[15] P. Guemas M. Beach and A.R. Nix, "Capacity and service extension for wireless networks using adaptive antennas," IEE Electronics Letters, vol. 30, no. 22, pp. 1813-1814, Oct 1994.

[16] G.J Foschini and M.J Gans, "On Limits of Wireless Communications in a Fading Environment when Using Multiple Antennas," Wireless Personal Commun., vol. 6, no. 3, pp. 311-335, March 1998.

[17] G.J Foschini, "Layered space-time architecture for wireless communications in fading environment when using multi-element antennas," Bell Labs Technical Journal, pp. 4159, Sept 1996.

[18] I.E. Telatar, "Capacity of multi antenna Gaussian channels," AT\&T Bell Labs. and Internal Tech. Memo, june 1995.

[19] J. M. Kahn C-N. Chuah, D. N. C. Tse and R. A. Valenzuela, "Capacity scaling in MIMO wireless systems under correlated fading," IEEE Transaction on Information Theory, vol. 48, no. 3, pp. 637-650, March 2002.

[20] M.A Wallace, J.W. Jensen, "Modeling the indoor MIMO wireless channel," Antennas and Propagation, IEEE Transactions on, vol. 50, no. 5, pp. 591-599, May 2002.

[21] H. Gore D.A. Paulraj A.J Gesbert, D. Bolcskei, "Outdoor MIMO wireless channels: models and performance prediction," Communications, IEEE Transactions on, vol. 50, no. 12, pp. 1926-1934, December 2002.

[22] D. Paulraj A.J Bolcskei, H. Gesbert, "On the capacity of OFDM-based spatial multiplexing systems," Communications, IEEE Transactions on, vol. 50, pp. 225-234, Feb 2002.

[23] H. Gore D.A. Paulraj A.J Gesbert, D. Bolcskei, "MIMO wireless channels: capacity and performance," In Proceedings of IEEE Global Telecommunications Conference, vol. 2, pp. 1083-1088, November 2000.

[24] A. M. Sayeed, "Deconstructing mutliantenna fading channels," IEEE Transactions on Signal Processing, vol. 50, no. 10, pp. 2563-2579, October 2002.

[25] J.B. Andersen, "Array gain and capacity for known random channels with multiple element arrays at both ends," Selected Areas in Communications, IEEE Journal on, vol. 18 , no. 11 , pp. $2172-2178$, Nov 2000 .

[26] D. Gore D. Gesbert, H. Bolcskei and A. Paulraj, "MIMO wireless channels: Capacity and performance prediction," Proceedings of IEEE Globecom Conference, vol. 2, pp. $1083-1088$, Nov. 2000.

[27] D.S. Shiu P.J. Smithi A. Naguib D. Gesbert, M. Shafi, "From Theory to Practice: An Overview of MIMO Space-Time Coded Wireless Systems," IEEE Journal on Selected Areas in Communications., vol. 21, no. 3, pp. 281-302, April 2003. 
[28] I.E. Telatar, "Capacity of multi antenna Gaussian channels," AT\&T Bell Labs. and Internal Tech. Memo, mars.bell-labs.com/papers/proof/, June 1995.

[29] A. Glavieux C. Berrou and P. Thitimajshima, "Near Shannon limit errorcorrecting coding and decoding: Turbo codes," In Proceedings of 1993 IEEE International Conference on Communications, Geneva, Switzerland, May 1993.

[30] R. Yates, "A framework for uplink power control in cellular radio systems," IEEE Journal on Selected Areas in Communications, vol. 43, no. 7, pp. 1341-1347, 1995.

[31] I. Katzela and M. Naghshineh, "Channel assignment schemes for cellular mobile telecommunication systems: A comprehensive survey," IEEE Personal Communications Magazine, vol. 3, no. 3, pp. 10-31, Jun 1996.

[32] L. C. Martorell R. Puigjaner I. F. Akyildiz, J. McNair and Y. Yesha, "Medium access control protocols for multimedia traffic in wireless networks," IEEE Network Magazine, vol. 13 , no. 4, pp. 39-47, Jul 1999.

[33] K. Bruninghaus H. Rohling, T. May and R. Grunheid, "Broad-band OFDM radio transmission for multimedia applications," Proceedings of the IEEE, vol. 87, no. 10, pp. 1778-1789, 1999.

[34] H. Zhang, "Service disciplines for guaranteed performance service in packet switching networks," Proceedings of the IEEE, vol. 83, no. 10, pp. 1374-1396, Oct 1995.

[35] Niranjan Joshi Achilles Kogiantis and M. Oguz Sunay, "On Transmit Diversity and Scheduling in Wireless Packet Data," Proceedings of the IEEE ICC'01 Conference, Helsinki, Finland, , no. 1, pp. 2433-2437, June 2001.

[36] R. Pankaj A. Jalali, R. Padovani, "Data throughput of CDMA-HDR a high efficiencyhigh data rate personal communication wireless system," Vehicular Technology Conference Proceedings, 2000. VTC 2000-Spring Tokyo. 2000 IEEE 51st, vol. 3, pp. 1854 1858, May 2000.

[37] D.N.C. Tse, "Optimal power allocation over parallel Gaussian channels," EECS Department, University of California, Berkeley, Technical Report No. UCB/ERL M99/ 1999, degas.eecs.berkeley.edu/ dtse/broadcast2.pdf.

[38] Dapeng Wu and Rohit Negi, "Downlink scheduling in a cellular network for qualityof-service assurance," Vehicular Technology, IEEE Transactions on, vol. 53, no. 5, pp. 1547-1557, Sep 2004.

[39] Lucent Technologies, "Throughput simulations for MIMO and transmit diversity enhancements to HSDPA," www.3gpp.org/ftp/tsg_ran/wgl_rll/TSGRI_l7/Docs/PDFs/R100-1388.pdf.

[40] T. Rappaport, "Wireless Communications," IEEE Press, 1996.

[41] John G. Proakis, "Digital Communications," (3rd edition, McGraw-Hill Inc. International Editions, 1995). 
[42] A. Stamoulis S.N. Diggavi, N. Al-Dhahir and A.R. Calderbank, "Great expectations: the value of spatial diversity in wireless networks," Proceedings of the IEEE, vol. 92, no. 2, pp. 219- 270, Feb 2004.

[43] W. Jakes, "Microwave Mobile Communications," IEEE Press, 1974.

[44] L.W. Barcley, "Propagation of Radio Waves," 2nd ed: IEE, 2003.

[45] A.F. Naguib G. Raleigh, S.N. Diggavi and A. Paulraj, "Characterization of fast fading vector channels for multi-antenna communication systems," Signals, Systems and Computers, 1994. 1994 Conference Record of the Twenty-Eighth Asilomar Conference on, vol. 2, pp. 853-857, Oct. 1994.

[46] I.A. Glover and P.M. Grant, "Digital Communications," Prentice Hall Europe, 1998.

[47] Sonny Erricson web site, “Technology: Understanding networks," www. sonyericsson.com.

[48] J.S. Blogh and L. Hanzo, "Third Generation Systems and Intelligent Wireless Networking: Smart Antennas and Adaptive Modulation," John Wiley \& Sons Inc. New York, 2002.

[49] A.K. Dinnis and J.S. Thompson, "Multiple antenna options for high data rate CDMA systems," Communications, IEE Proceedings, vol. 152, no. 4, Aug 2005.

[50] R.G. Gallager, "Information Theory and Reliable Communications," John Wiley and Sons, New York 1968.

[51] T.M. Cover and J.A. Thomas, "Elements of Information Theory," John Wiley and Sons, Inc., New York 1991.

[52] D. Brennan, "Linear diversity combining techniques," Proceedings IEEE 47, pp. 10751102, Jun 1959.

[53] David Gesbert and Jabran Akhtar, "Breaking the barriers of Shannon's capacity: An overview of MIMO wireless systems," . www.eurecom.fr/ gesbert/papers/TelektronikkMIMO.pdf.

[54] A. Paulraj and C.B. Papadias, "Space-time processing for wireless communications," IEEE Signal Processing Magazine, vol. 14, pp. 49-83, Nov 1997.

[55] G.D. Golden P.W. Wolniansky, G.J Foschini and R.A. Valenzuela, "Detection algorithm and initial laboratory results using VBLAST space-time communication architecture," IEE Electronic Letters, vol. 35, no. 1, pp. 14-16, January 1999.

[56] R.A. Valenzuela G.J. Foschini, G.D. Golden and P.W. Wolniansky, "Simplified processing for high spectral efficiency wireless communication employing multi-element arrays," Vehicular Technology, IEEE Transactions on, vol. 17, no. 11, pp. 1841-1852, Nov 1999.

[57] C.E. Shannon, "A mathematical theory of communications," Bell Systems Technical Journal 27, pp. 623-656, 1948. 
[58] T. Ericson, "A Gaussian channel with slow fading," IEEE Transactions of information theory 16, pp. 353-356, May 1970.

[59] S. Shamai L.H. Ozarow and A.D. Wyner, "Information theoretic considerations for cellular mobile radio," Vehicular Technology, IEEE Transactions on, vol. 43, no. 2, pp. 359-378, May 1994.

[60] C.E. Shannon, "Channels with side-information at the transmitter," IBM Journal of Research and Development 2, pp. 289-293, Oct 1958.

[61] S.I. Gelfand and M.S. Pinsker, "Coding for channels with random parameters," Probl. Pered. Inform. (Probl. Pered. Trans.) 9, vol. 1, pp. 19-31, 1980.

[62] J. Proakis E. Biglieri and S. Shamai, "Fading channels: information-theoretic and communications aspects," Information Theory, IEEE Transactions on, vol. 44, no. 6, pp. 2619-2692, Oct 1998.

[63] F.D. Neeser and J.L. Massey, "Proper complex random processes with applications to information theory," Information Theory, IEEE Transactions on, vol. 39, no. 4, pp. 1293-1302, Jul 1993.

[64] N. Seshadri V. Tarokh and A.R. Calderbank, "Space-time codes for high data rate wireless communication: performance criterion and code construction," Information Theory, IEEE Transactions on, vol. 44, no. 2, pp. 744-765, March.

[65] B.M. Hochwald T.L. Marzetta, "Capacity of a mobile multiple-antenna communication link in Rayleigh flat fading," Information Theory, IEEE Transactions on, vol. 45, no. 1, pp. 139 - 157, Jan 1999.

[66] I.E. Telatar, "Capacity of multi antenna Gaussian channels," European. Trans. Telecommun., vol. 10, no. 6, pp. 585-595, 1999.

[67] P.B. Rapajic and D. Popescu, "Information capacity of a random signature multipleinput multiple-output channel," Communications, IEEE Transactions on, vol. 48, pp. 1245-1248, Aug 2000.

[68] F.R. Farrokhi A. Lozano and R.A. Valenzuela, "Lifting the limits on high speed wireless data access using antenna arrays," Communications Magazine, IEEE, vol. 39, pp. 156162, Sept 2001.

[69] P.F. Driessen and G.I. Foschini, "On the capacity formula for multiple input-multiple output wireless channels: a geometric interpretation," Communications, IEEE Transactions on, vol. 47, no. 2, pp. 173-176, Feb 1999.

[70] A. M. Sengupta and P. P. Mitra, "Capacity of multivariate channels with multiplicative noise: I. random matrix techniques and large-n expansions for full transfer matrices," xxx.lanl.gov/abs/physics/0010081, Oct 2000.

[71] B. Rimoldi N. Chiurtu and E. Telatar, "Dense multiple antenna systems," Information Theory Workshop, 2001. Proceedings. 2001 IEEE, pp. 108-109, Sep 2001. 
[72] D. Goeckel S. Wei and R. Janaswamy, "On the capacity of fixed length linear antenna arrays under bandlimited correlated fading," Princeton, NJ: Princeton University, Apr 2002.

[73] G.L. Stuber, "Principles of Mobile Communications, 2nd ed.," Kluwer Academic Publishers, 200.

[74] L. Li and A. Goldsmith, "Optimal Resource Allocation for Fading Broadcast ChannelsPart I: Ergodic Capacity," IEEE Transactions on Communications, vol. 47, no. 3, pp. 1083-1102, Mar 2001.

[75] G. Caire and S. Shamai, "On the achievable throughput of a multiantenna Gaussian broadcast channel," Information Theory, IEEE Transactions on, vol. 49, no. 7, pp. 16911701, Jul 2003.

[76] N. Jindal S. Vishwanath and A. Goldsmith, "Duality, achievable rates, and sum-rate capacity of Gaussian MIMO broadcast channels," Information Theory, IEEE Transactions on, vol. 49, no. 10, pp. 2658-2668, Oct 2003.

[77] P. Viswanath and D.N.C. Tse, "Sum capacity of the vector Gaussian broadcast channel and uplink-downlink duality," Information Theory, IEEE Transactions on, vol. 49, no. 8, pp. 1912-1921, Aug 2003.

[78] R.M. Tranter W.H. Jing Jiang, Buehrer, "Antenna diversity in multiuser data networks," Communications, IEEE Transactions on, vol. 52, no. 3, pp. 490 - 497, March 2004.

[79] D.N.C. Tse, "Multiuser diversity in wireless networks," Wireless Communications Seminar, Stanford University, www.eecs.berkeley.edw/ dtse/ima810.pdf, April 2001.

[80] R. Knopp and P. Humblet, "Information capacity and power control in single cell multiuser communications," Communications, 1995. ICC 95 Seattle, Gateway to Globalization, 1995 IEEE International Conference on, vol. 1, pp. 331 - 335, June 1995.

[81] M. Grob R. Padovani N. Sindhushyana P. Bender, P. Black and S. Viterbi, "CDMA/HDR: a bandwidth efficient high speed wireless data service for nomadic users," Communications Magazine, IEEE, vol. 38, no. 7, pp. 70-77, Jul 2000.

[82] S. Keshav A. Demers and S. Shenker., "Analysis and simulation of a fair queueing algorithm," Journal of Internetworking Research and Experience, vol. 1, pp. 3-26, Oct. 1990.

[83] A. Parekh and R. Gallager., "A generalized processor sharing approach to flow control in integrated services networks: the single-node case," IEEE/ACM Transactions on Networking, vol. 1, pp. 3-26, 1993.

[84] H. Zhang., "Service disciplines for guaranteed performance service in packetswitching networks," Proceedings of the IEEE, vol. 83, no. 10, pp. 1374-1396, Oct. 1995.

[85] Elliott R.C. et al., "Scheduling Algorithms for the CDMA2000 Packet Data Evolution.," Vehicular Technology Conference, 2002. VTC 2002-Fall, vol. 1, pp. 304-310, Sep 2002. 
[86] W. Ajib and D. Haccoun, "An overview of scheduling algorithms in mimo-based fourthgeneration wireless systems," Network, IEEE, vol. 19, no. 5, pp. $43-48$, Sep-Oct 2005.

[87] Liu Youjian Vincent K.N. Lau and Tai Ann Chen, "Optimal multi-user space time scheduling for wireless communications," Vehicular Technology Conference, 2002. Proceedings. VTC 2002-Fall. 2002 IEEE 56th, vol. 4, pp. 1939 - 1942, Sep 2002.

[88] V.K.N. Lau and Yu-Kwong Kwok, "Charisma: a novel channel-adaptive tdma-based multiple access control protocol for integrated wireless voice and data services," WCNC 2000 IEEE Wireless Communications and Networking Conference, vol. 2, pp. 507-511, Sep 2000.

[89] Holtzman J.M., "CDMA Forward Link Waterfilling Power Control.," Vehicular Technology Conference, 2000. VTC 2000-Spring Tokyo. 2000 IEEE 51st, vol. 3, pp. 1663-1667, May 2000.

[90] Elliott R.C. and W.A. Krzymien, "Scheduling algorithms for the high-throughput packet data service in cellular radio systems," Electrical and Computer Engineering, Canadian Journal of, vol. 29, no. 1, pp. $117-127,2004$.

[91] Kolding T. et al., "Performance Aspects of WCDMA Systems with High Speed Downlink Packet Access (HSDPA).," Vehicular Technology Conference, 2002. VTC 2002-Fall, vol. 1, pp. 477- 481, 2002.

[92] Liu X., "Opportunistic Transmission Scheduling With Resource-Sharing Constraints in Wireless Networks.," Selected Areas in Communications, Journal on, vol. 19, pp. 2053-2064, Oct. 2001.

[93] Hosein P., "QoS Control for WCDMA High Speed Data.," Mobile and Wireless Communications Network, 4th International Workshop on, pp. 169-173, September 2002.

[94] Motorola, "Evaluation Methods for High Speed Downlink Packet Access," TSG RI $14(00) 909$.

[95] A.K. Dinnis and J.S. Thompson, "Investigation of faimess issues with multiple antennas in high data rate CDMA systems," Vehicular Technology Conference, 2004. VTC 2004Spring. 2004 IEEE 59th, vol. 2, pp. 723 - 727, May.

[96] Tae Hyung Kim Jong Hun Rhee and Dong Ku Kim, "Evaluation Methods for High Speed Downlink Packet Access," Vehicular Technology Conference, 2001. VTC 2001 Fall. IEEE VTS 54th, vol. 2, pp. 743 - 746, Oct. 2001.

[97] J. Wigard P. Ameigeiras and P. Mogensen, "Performance of packet scheduling methods with different degree of faimess in HSDPA," Vehicular Technology Conference, VTC2004-Fall. 2004 IEEE 60th, vol. 2, pp. 860-864, Sep 2004.

[98] D. Bertsekas and R. Gallager, "Data Networks. Second Edition.", Prentice-Hall, 1992.

[99] Berger L. et al., "Interaction of Transmit Diversity and Proportional Fair Scheduling.," Vehicular Technology Conference, 2003. VTC 2003 Spring., vol. 4, pp. 2423-2427, 2003. 
[100] Li-Chun Wang and Ming-Chi Chen, "Comparisons of link-adaptation-based scheduling algorithms for the WCDMA system with high-speed downlink packet access," Electrical and Computer Engineering, Canadian Journal of, vol. 29, no. 1, pp. 109 - 116, Jan-Apr 2004.

[101] Holtzman J.M., "Asymptotic Analysis of the Proportional Fair Algorithm.," Personal, Indoor and Mobile Radio Communications, 2001 12th IEEE International Symposium on, vol. 2, pp. F-33-F-37, 2001.

[102] Kelly F., "Charging and Rate Control for Elastic Traffic.," Europeans Transactions on Telecommunications, vol. 8, pp. 33-37, 1997.

[103] M.J. Gans D. Chizhik, G.J. Foschini and R.A. Valenzuela, "Keyholes, correlations, and capacities of multi-element transmit and receive antennas," IEEE Transactions on Wireless Communications, vol. 1, no. 2, pp. 78 -82, April 2002.

[104] J.P. Kermoal K.I. Pedersen, J.B. Andersen and P. Mogensen, "A stochastic multi-input multi-output radio channel model for evaluation of space-time coding algorithms," IEEE VTC Fall 2000, vol. 2, pp. 893-897, Sept 2000.

[105] A.F. Molisch, "A channel model for MIMO systems in macro- and microcellular enviroments," IEEE VTC Spring 2002, vol. 2, pp. 655-659, May 2002.

[106] D. S. Shiu, "Wireless Communication Using Dual Antenna Array.," Norwell, MA: Kluwer, 2000.

[107] K.I. Pedersen J.P. Kermoal, L. Schumacher and F. Frederiksen P.E. Mogensen, "A stochastic MIMO radio channel model with experimental validation," IEEE Journal on Selected Areas in Communications., vol. 20, no. 6, pp. 1211-1226, August 2002.

[108] C.K. Rushforth Z. Xie, R.T. Short, "A family of suboptimum detectors for coherent multiuser communications," IEEE Journal on Selected Areas in Communications, vol. 8, no. 4, pp. 683-690, May 1990.

[109] S. Verdu M. Honig, U. Madhow, "Blind adaptive multiuser detection," IEEE Trans. Inform. Theory, vol. 41, no. 4, pp. 994-960, Jul. 1995.

[110] G.D. Golden P.W. Wolniansky, G.J Foschini and R.A. Valenzuela, "V-BLAST: An architecture for realizing very high data rates over the rich-scattering wireless channel," URSI International Symposium on Signals, Systems and Electronics, pp. 295-300, 1999.

[111] L. Mogensen P.E. Pedersen K.I. Kermoal, J.P. Schumacher, "Experimental investigation of correlation properties of MIMO radio channels for indoor picocell scenarios," Vehicular Technology Conference, 2000. IEEE VTS-Fall VTC 2000. 52nd, vol. 1, pp. 14-21, Sept 2000.

[112] B. McNamara D. Karlsson P. Beach M Kai Yu Bengtsson, M. Ottersten, "Modeling of wide-band MIMO radio channels based on NLOS indoor measurements," Vehicular Technology, IEEE Transactions on, vol. 53, no. 3, pp. 655-665, May 2004.

[113] S. M. Kay, ," Fundamentals of Statistical Signal Processing: Estimation Theory, vol. 1, Prentice Hall PTR 1993. 
[114] M.J. Kahn J.M Da-Shan Shiu Foschini, G.J. Gans, "Fading correlation and its effect on the capacity of multielement antenna systems," Communications, IEEE Transactions on, vol. 48, no. 3, pp. 502-513, March 2000.

[115] A. F. Molisch, "A generic model for MIMO wireless propagation channels in macroand microcells," Signal Processing, IEEE Transactions on, vol. 52, no. 1, pp. 61-71, January 2004.

[116] M. Abdi, A. Kaveh, "A space-time correlation model for multielement antenna systems in mobile fading channels," Selected Areas in Communications, IEEE Journal on, vol. 20, no. 3, pp. 550-560, April 2002.

[117] B. Ottersten D. McNamara P. Karlson K. Yu, M. Bengstsson and M. Beach, "A wideband statistical model for NLOS indoor MIMO channels.," In Proceedings of IEEE Vehicular Technology Conference, vol. 1, pp. 370-374, IEEE VTC Spring 2002.

[118] B. McNamara D. Karlsson P. Beach M. Kai Yu Bengtsson, M. Ottersten, "Second order statistics of NLOS indoor MIMO channels based on 5.2 ghz measurements," Global Telecommunications Conference, 2001. GLOBECOM 'O1. IEEE, vol. 1, pp. 156-160, November 2001.

[119] "IST-1999-11729 METRA Project," www.ist-imetra.org/metra/, 2001.

[120] M.A. Fletcher P.N. Karlsson P. McNamara, D.P. Beach, "Initial investigation of multipleinput multiple-output (MIMO) channels in indoor environments," Communications and Vehicular Technology, 2000. SCVT-200. Symposium on, pp. 139-142, October 2000.

[121] Geoffrey J. Byers and Fambirai Takawira, "The influence of spatial and temporal correlation on the capacity of MIMO channels," Wireless Communications and Networking, 2003. WCNC 2003. 2003 IEEE, vol. 1, pp. 359 - 364, March 2003.

[122] B.H. Fleury, "First- and second-order characterization of direction dispersion and space selectivity in the radio channel," IEEE Trans. Inform. Theory, vol. 46, pp. 2027-2044, Sept. 2000.

[123] W.C.Y. Lee, "Effects on correlation between two mobile radio basestation antennas," IEEE Trans. Commun., vol. 21, pp. 1214-1024, Nov. 1973.

[124] J. Salz and J. Winters, "Effects of fading correlation on adaptive arrays in digital mobile radio," IEEE Trans. Veh. Technol., vol. 43, pp. 1049-1057, Nov. 1994.

[125] C.F. Van Loan G.H. Golub, "Matrix Computations," Johns Hopkins University Press, 1983.

[126] W. Wu and K. Chen, "Linear multiuser detectors for sychronous CDMA communication over rayleigh fading channels," IEEE International Symposium, Personal, Indoor and Mobile Radio Communiacations, PIRMC'96, Seventh, vol. 2, pp. 578-582, Oct 1996.

[127] Aneta Pavlic Stephen Baro, Gerhard Bauch and Andreas Semmler, "Improving BLAST performance using space-time block codes and turbo decoding," GLOBECOM 2000, vol. 2, pp. 1067-1071, January 72000. 
[128] M. Sellathurai and S. Haykin, "A simplified diagonal blast architecture with iterative parallel-interference cancelation receivers," IEEE International Conference on Communications, vol. 10, pp. 3067-3071, 2001.

[129] M. Sellathurai and S. Haykin, "Turbo-blast for high-speed wireless communications," Wireless Communications and Networking Conference, vol. 1, pp. 315-320, 2000.

[130] R. Gozali, "Space-time codes for high data rate wireless communications," Ph.D dissertation Virginia Polytechnic Institute and State University, 2002.

[131] G.D. Golden et al., "Detection algorithm and initial laboratory results using VBLAST spacetime communication architecture," Electron. Lett., vol. 35, no. 1, pp. 14-16, January 71999.

[132] A. Klein, "Multi-user detection of CDMA signals-algorithms and their application to cellular mobile radio," Fortschritt-Berichte VDI, 1996.

[133] Papoulis Athanasions, "Probability, Random Variables and Stochastic Processes," McGraw-Hill, 1984.

[134] MAXIM, "HFTA-05.0: Statistical Confidence Levels for Estimating BER Probability," www.maxim-ic.com/appnotes.cfm/appnote _number/1095.

[135] M. Marcus and H. Minc, "Introduction to Linear Algebra," New York: Dover, 1988.

[136] M. Airy R.W. Heath and A.J. Paulraj, "Multiuser diversity for MIMO wireless systems with linear receivers," Proc, Asilomar Conf. Signals, Systems, and Computers, Pacific Grove, CA, pp. 1194-1199, Nov. 2001.

[137] Oh-Soon Shin and Kwang Bok(ED) Lee, "Packet Scheduling for MIMO Cellular Systems," IEEE Vehicular Technology Conference, pp. 1694-1698, Apr. 2003.

[138] D.N.C. Tse P. Viswanath and R. Laroia, "Opportunistic beamforming using dumb antennas," IEEE Trans. Inform. Theory, vol. 48, no. 6, pp. 1277-1294, Jun. 2002.

[139] Proakis J. Biglieri, E. and S. Shamai, "Fading channels: information-theoretic and communications aspects," Information Theory, IEEE Transactions on, vol. 44, no. 6, pp. $2619-2692$, Oct. 1998.

[140] Lang Tong Adireddy, S. and H. Viswanathan, "Optimal placement of training for frequency-selective block-fading channels," Information Theory, IEEE Transactions on, vol. 48 , no. 8, pp. $2338-2353$, Aug. 2002.

[141] S. Sandhu R.W. Heath and A.J. Paulraj, "Antenna selection for spatial multiplexing system with linear receivers," IEEE Commun. Lett., pp. 142-144, Apr. 2001.

[142] Steenkiste P. Eckhardt, D.A., "Effort-limited fair (ELF) scheduling for wireless networks," INFOCOM 2000. Nineteenth Annual Joint Conference of the IEEE Computer and Communications Societies. Proceedings. IEEE, vol. 3, pp. 1097 - 1106, March 2000.

[143] Ahmed M. Rohani K. Pautler, J., "On application of multiple-input multiple-output antennas to CDMA cellular systems," Vehicular Technology Conference, 2001. VTC 2001 Fall. IEEE VTS 54th, vol. 3, pp. 1508 - 1512, Oct. 2001. 
[144] Kolding T.E., "Link and system performance aspects of proportional fair scheduling in WCDMA/HSDPA," Vehicular Technology Conference, 2003. VTC 2003-Fall. 2003 IEEE 58th, vol. 3, pp. 1717 - 1722, Oct. 2003.

[145] B. Mukherjee, "On the infinite buffer model and the implementation aspects of the pipersistent protocol for unidirectional broadcast bus networks," Communications, 1988. ICC 88. Digital Technology - Spanning the Universe. Conference Record. IEEE International Conference on, vol. 1, pp. 273 - 277, June 1998.

[146] Arogyaswami Paulraj, "Integrating Space-Time Processing into Wireless Communications," www.isi.edu/workshop/public_html/wmcw97/paulraj.html. 


\section{Appendix A \\ Pseudocodes for Chapter 4}

1: $k u=0$

2: $s u=$ number of simultaneous users

3: for all time slots $t$ do

4: $\quad$ for $n=1$ to $N_{T}$ do

5: $\quad k u=k u+1$

6: $\quad$ if $k u>s u$ then

7: $\quad k u=1$

8: $\quad$ end if

9: $\quad S U G(n)=k u$ apply RRS scheme to create

Scheduled User Group (SUG) for time slot $t\}$

10: end for $\{n$th transmit antenna $\}$

11: for all users $k$ such that $k \in S U G$ do

12: $\quad\left\{\gamma_{k, 1}(t), \cdots, \gamma_{k, N_{T}}(t), D_{S U G(k), 1}(t), \cdots, D_{S U G(k), N_{T}}(t)\right\}=\operatorname{vblast\_ sinr}(k)$ \{vblast_sinr (Figure (A.2)) calculates the SINR information after VBLAST detection\}

13: end for $\{k$ th user $\}$

14: $\quad \boldsymbol{D}(t)=\left\{\boldsymbol{D}_{S U G(1), 1}(t), \cdots, \boldsymbol{D}_{S U G(1), N_{T}}(t), \cdots, \boldsymbol{D}_{S U G\left(N_{T}\right), 1}(t), \cdots, \boldsymbol{D}_{S U G\left(N_{T}\right), N_{T}}(t)\right\}$

15: integrate all the possible one-to-one mappings between the $N_{T}$ scheduled users (SUG) and $N_{T}$ transmit antennas, into a matrix $\boldsymbol{P}^{N_{T} ! \times N_{T}}(t)\left\{\boldsymbol{P}_{23}(t)=4=>\right.$ according to the 2nd mapping, the user 4 is assigned to antenna 3$\}$

16: $\quad$ for $n=1$ to $N_{T}$ ! do

17: $\quad C_{n}^{A A-R R S-V B L A S T}(t)=$ Feasibility_Test $\left(n, N_{T}, \boldsymbol{P}(t), \boldsymbol{D}(t), \Gamma(t)\right)$ \{calculate the system capacity for the $n$th mapping sequence, the Feasibility Test() (Figure (A.3))function is discussed thoroughly in section 4.4.2\}

18: end for $n$th mapping sequence - exhaustive search

19: apply eqn. 4.13 to find the mapping sequence best.n that realizes the highest system capacity $\max _{-} C^{A A-R R S-V B L A S T}(t)$

20: end for $\{$ time slots $t\}$

Figure A.1: VBLAST AA-RRS algorithm 
1: apply eqn. 4.1 to calculate $\boldsymbol{H}_{k, 1}(t)$

2: apply eqn. 4.4 to calculate $\boldsymbol{W}_{k, 1}(t)$ \{VBLAST initialization $\}$

3: for $i=1$ to $N_{T}$ do

4: $\quad$ find the transmit antenna $n$ that has been detected by the VBLAST detector after the $i$ th iteration by the $k$ th user

5: $\quad$ if $i>1$ then

6: $\quad$ update $D_{k, n}(t)$ vector (eqn. (4.9)), $d_{k, i-1}(t)=$ det_ant

7: $\quad$ apply eqn. 4.8 to calculate $\gamma_{k, n}(t)$ \{VBLAST detector with MMSE nulling

8: $\quad$ det_ant $=n$

9: else

10: $\quad$ det_ant $=n$

11: apply eqn. 4.6 to calculate $\gamma_{k \text {,det_ant }}(t)$ \{MMSE detector\}

12: end if

13: $\quad$ if $i<N_{T}$ then

14: $\boldsymbol{H}_{k, i+1}(t)=\boldsymbol{H}_{\bar{k}, i}^{+}(t)$ \{create $\boldsymbol{H}_{k, i+1}(t)$, which is a 'deflated' version of $\boldsymbol{H}_{k, i}(t)$, in which columns $d_{k, 1}(t), d_{k, 2}(t), \ldots, d_{k, i-1}(t)$, det_ant have been set to zero

15: $\quad$ apply eqn. 4.4 to calculate $W_{k, i+1}(t)$

16: end if

17: end for $\{$ ith VBLAST iteration for $k$ th user $\}$

Figure A.2: vblast_sinr( $k$ user $)$

1: for ant $=1$ to $N_{T}$ do

2: $\quad u=p_{n, a n t}$ \{according to the $n$th mapping, transmit antenna ant is assigned to user $u$ \}

3: $\quad$ for $d t=1$ to $N_{T}$ do

4: $\quad d t_{-} a n t=d_{u, d t}\left\{d t_{-} a n t\right.$ is the detected antenna from user $u$ after the $d t$ th iteration of VBLAST algorithm \}

5: $\quad$ if $d t$ ant $\neq$ ant then

6: $\quad u_{-} d t_{-} a n t=p_{n, d t_{-} a n t}$ if the detected antenna $d t_{-} a n t$ is not ant then $u_{-} d t_{-} a n t$ is allocated to $\left.d t \_a n t\right\}$

7: $\quad C_{d t_{\text {ant }}}=\log _{2}\left(1+\min \left(\gamma_{u \_d t \_a n t, d t \_a n t}, \gamma_{u, d t \_a n t}\right)\right)$

8: $\quad$ else

9: $\quad C_{\text {ant }}=\log _{2}\left(1+\gamma_{u, \text { ant }}\right)$

10: break

11: $\quad$ end if

12: end for

13: end for

Figure A.3: Feasibility_Test() 
1: $k u=0$

2: $s u=$ number of simultaneous users

3: for all time slots $t$ do

4: $\quad$ for $n=1$ to $N_{T}$ do

5: $\quad k u=k u+1$

6: $\quad$ if $k u>s u$ then

7: $\quad k u=1$

8: $\quad$ end if

9: $\quad S U G(n)=k u$ apply RRS scheme to create

Scheduled User Group (SUG) for time slot $t\}$

10: end for $\{n$th transmit antenna $\}$

11: for all users $k$ such that $k \in S U G$ do

12: $\quad$ apply eqn. 4.1 to calculate $\boldsymbol{H}_{k}(t)$

13: $\quad$ apply eqn. 4.4 to calculate $\boldsymbol{W}_{k}(t)$ \{MMSE initialization

14: $\quad$ for $n=1$ to $N_{T}$ do

15: $\quad$ apply eqn. 4.6 to calculate $\gamma_{k, n}(t)$ (MMSE detector)

16: end for $n$th MMSE iteration for $k$ th user

17: end for $\{k$ th user $\}$

18: $\quad$ for $n=1$ to $N_{T}$ do

19: best_ $u_{n}=\max _{k \in S U G} \gamma_{k, n}(t)$ for the $n$th transmit antenna find the user user that gives the highest SINR when receiving from antenna $n$, and store this SINR value in best_ $\left.u_{n}\right\}$

20: end for $\{n$th transmit antenna $\}$

21: $\quad C^{A A-R R S-B e s t-U s e r}(t)=\sum_{n=1}^{N_{T}} \log _{2}\left(1+\right.$ best_un $\left.u_{n}\right)$

22: end for $\{$ time slot $t\}$

Figure A.4: AA-RRS best user Algorithm 


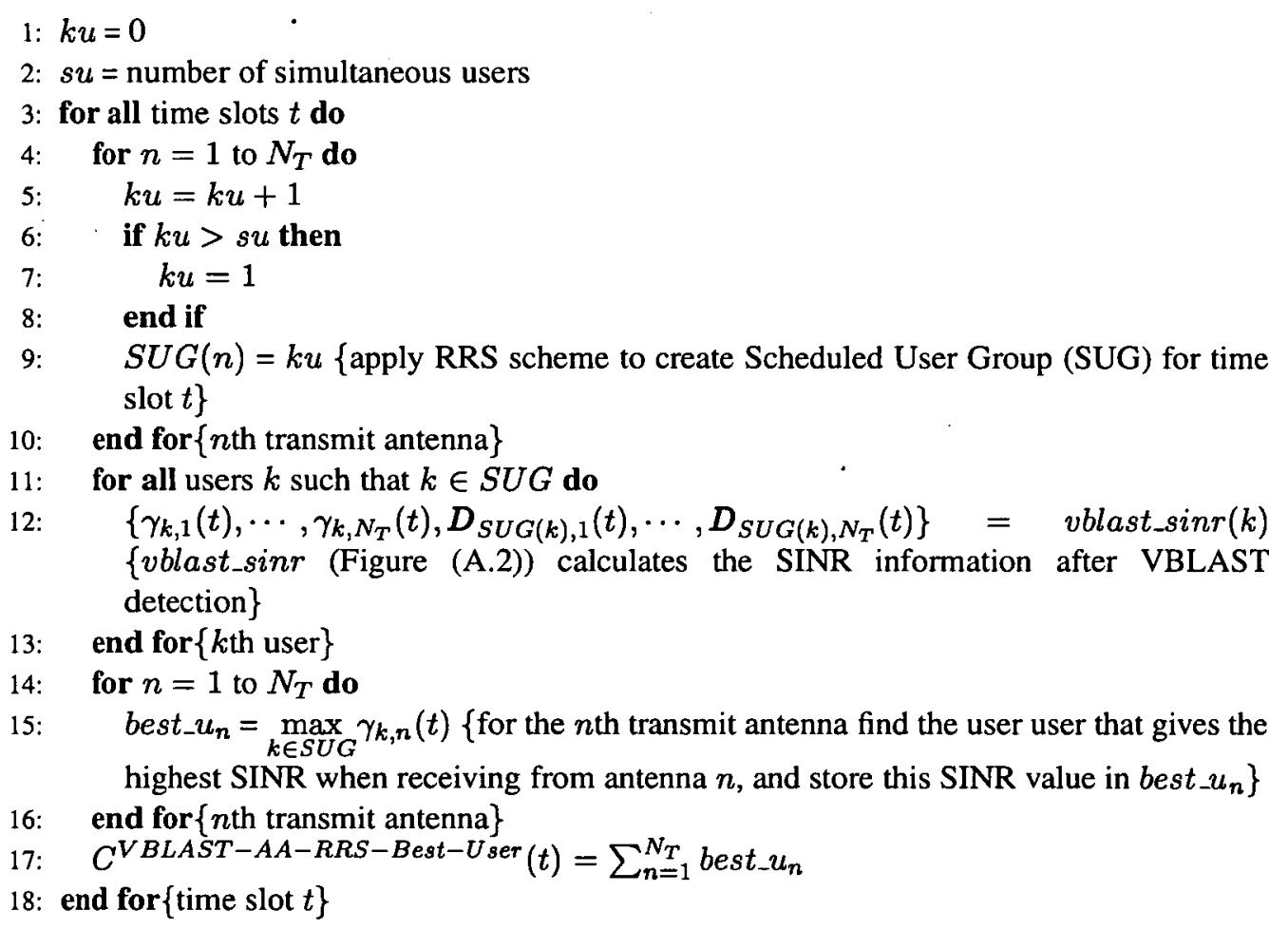

Figure A.5: VBLAST AA-RRS best user Algorithm 
1: $s u=$ number of simultaneous users

2: $U G S V=\{1,2, \ldots$, su $\}$ \{Users Group (UGSV) to be SerVed $\}$

3: $S V U G=\{\}\{$ the SerVed User Group (SVGU) is an empty set $\}$

4: for all time slots $t$ do

5: $\quad k u=0$ \{number of users in SUG\}

6: $\quad$ for $u=1$ to $s u$ do

7: $\quad$ if $u \in(U G S V-U G S V \cap S V U G)$ then

8: $\quad k u=k u+1$

9: $\quad S U G(k u)=u$ \{create the Scheduled User Group (SUG) for time slot $t$ \}

10: end if

11: end for $\{u$ th user $\}$

12: $\quad$ if $k u<N_{T}$ then

13: $\quad t m p=k u$

14: for $k u=(t m p+1)$ to $N_{T}$ do

15: $\quad S U G(k u)=S U G(k u-t m p)$

16: end for $\{k u$ th position in the SUG $\}$

17: end if

18: for all users $k$ such that $k \in S U G$ do

19: $\quad$ apply eqn. 4.1 to calculate $\boldsymbol{H}_{k}(t)$

20: $\quad$ apply eqn. 4.4 to calculate $\boldsymbol{W}_{k}(t)$ \{MMSE initialization $\}$

21: $\quad$ for $n=1$ to $N_{T}$ do

22:

23: apply eqn. 4.6 to calculate $\gamma_{k, n}(t)$ (MMSE criterion) end for $n$th MMSE iteration for $k$ th user

24:

25: $\max _{-} \gamma_{k}(t)=\max _{n \in\left\{1, \ldots, N_{T}\right\}} \gamma_{k, n}(t)$ \{maximum SINR for $k$ th user $\}$

end for $\{k$ th user $\}$

26: $\quad$ sorted_max- $\gamma(t)=\left\{\max _{-} \gamma_{k_{1}}(t), \ldots, \max _{-} \gamma_{k_{k u}}(t)\right\}\left\{\right.$ where $\max _{-} \gamma_{k_{1}}(t) \leq \ldots \leq$ $\left.\max _{-} \gamma_{k_{k u}}(t)\right\}$

27: $\quad$ sorted_k $=\left\{\left\{k_{1}, k_{2}, \ldots, k_{k u}\right\}\left\{\right.\right.$ where $\left.\max _{-} \gamma_{k_{1}}(t) \leq \ldots \leq \max _{-} \gamma_{k_{k u}}(t)\right\}$

28: $\quad$ SVUG $=\left\{\left\{k_{1}, k_{2}, \ldots, k_{N_{T}}\right\}\{\right.$ create the SerVed User Group SVUG $\}$

29: $\quad C^{A A-M M S E-f a i r}(t)=\sum_{n=1}^{N_{T}} \log _{2}\left(1+\right.$ sorted_max $\left.\gamma_{n}(t)\right)$ system capacity for AA MMSE fair scheme\}

30: end for $\{$ time slot $t\}$

Figure A.6: AA MMSE fair Algorithm 
1: $s u=$ number of simultaneous users

2: $U G S V=\{1,2, \ldots$, su $\}$ UUsers Group (UGSV) to be served $\}$

3: $S V U G=\{\}$ the SerVed User Group (SVGU) is an empty set $\}$

4: for all time slots $t$ do

5: $k u=0$ \{number of users in SUG

6: $\quad$ for $u=1$ to $s u$ do

7: $\quad$ if $u \in(U G S V-U G S V \cap S V U G)$ then

8: $\quad k u=k u+1$

9: $\quad S U G(k u)=u$ \{create the Scheduled User Group (SUG) for time slot $t\}$

10: $\quad$ end if

11: end for $\{u$ th user\}

12: $\quad$ if $k u<N_{T}$ then

13: $\quad t m p=k u$

14: $\quad$ for $k u=(t m p+1)$ to $N_{T}$ do

15: $\quad S U G(k u)=S U G(k u-t m p)$

16: end for $\{k u$ th position in the SUG

17: end if

18: $\quad$ for all users $k$ such that $k \in S U G$ do

19: $\left\{\gamma_{k, 1}(t), \cdots, \gamma_{k, N_{T}}(t), D_{S U G(k), 1}(t), \cdots, D_{S U G(k), N_{T}}(t)\right\}=$ vblast_sinr $(k)$ \{vblast_sinr (Figure (A.2)) calculates the SINR information after VBLAST detection\}

20: end for $\{k$ th user $\}$

21: $\quad \boldsymbol{D}(t)=\left\{\boldsymbol{D}_{S U G(1), 1}(t), \cdots, \boldsymbol{D}_{S U G(1), N_{T}}(t), \cdots, \boldsymbol{D}_{S U G\left(N_{T}\right), 1}(t), \cdots, \boldsymbol{D}_{S U G\left(N_{T}\right), N_{T}}(t)\right\}$

22: integrate all the possible one-to-one mappings between the $N_{T}$ scheduled users (SUG) and $N_{T}$ transmit antennas, into a matrix $\boldsymbol{P}^{N_{T} ! \times N_{T}}(t)\left\{\boldsymbol{P}_{23}(t)=4=>\right.$ according to the 2nd mapping, the user 4 is assigned to antenna 3 \}

23: $\quad$ for $n=1$ to $N_{T}$ ! do

24: $\quad C_{n}^{A A-R R S-V B L A S T}(t)=$ Feasibility_Test $\left(n, N_{T}, \boldsymbol{P}(t), \boldsymbol{D}(t), \Gamma(t)\right)$ \{calculate the system capacity for the $n$th mapping sequence, the Feasibility $T$ est () (Figure (A.3)) function is discussed thoroughly in section 4.4.2\}

25: end for $\{n$th mapping sequence - exhaustive search

26: $\quad\left\{C^{A A-V B L A S T-f a i r}(t), n_{\text {opt }}\right\}=\max _{n} C_{n}^{A A-R R S-V B L A S T}(t)$ find the maximum feasible system capacity and the $n_{\text {opt }}$ th mapping sequence that achieves this capacity. $\}$

27: $\quad$ SVUG $=\left\{\boldsymbol{P}_{n_{\text {opt }} 1}, \boldsymbol{P}_{n_{\text {opt }} 2}, \cdots, \boldsymbol{P}_{n_{\text {opt }} N_{T}}\right\}$

28: end for $\{$ time slot $t\}$

Figure A.7: AA VBLAST fair Algorithm 


\section{Appendix B \\ Pseudocodes for Chapter 5}

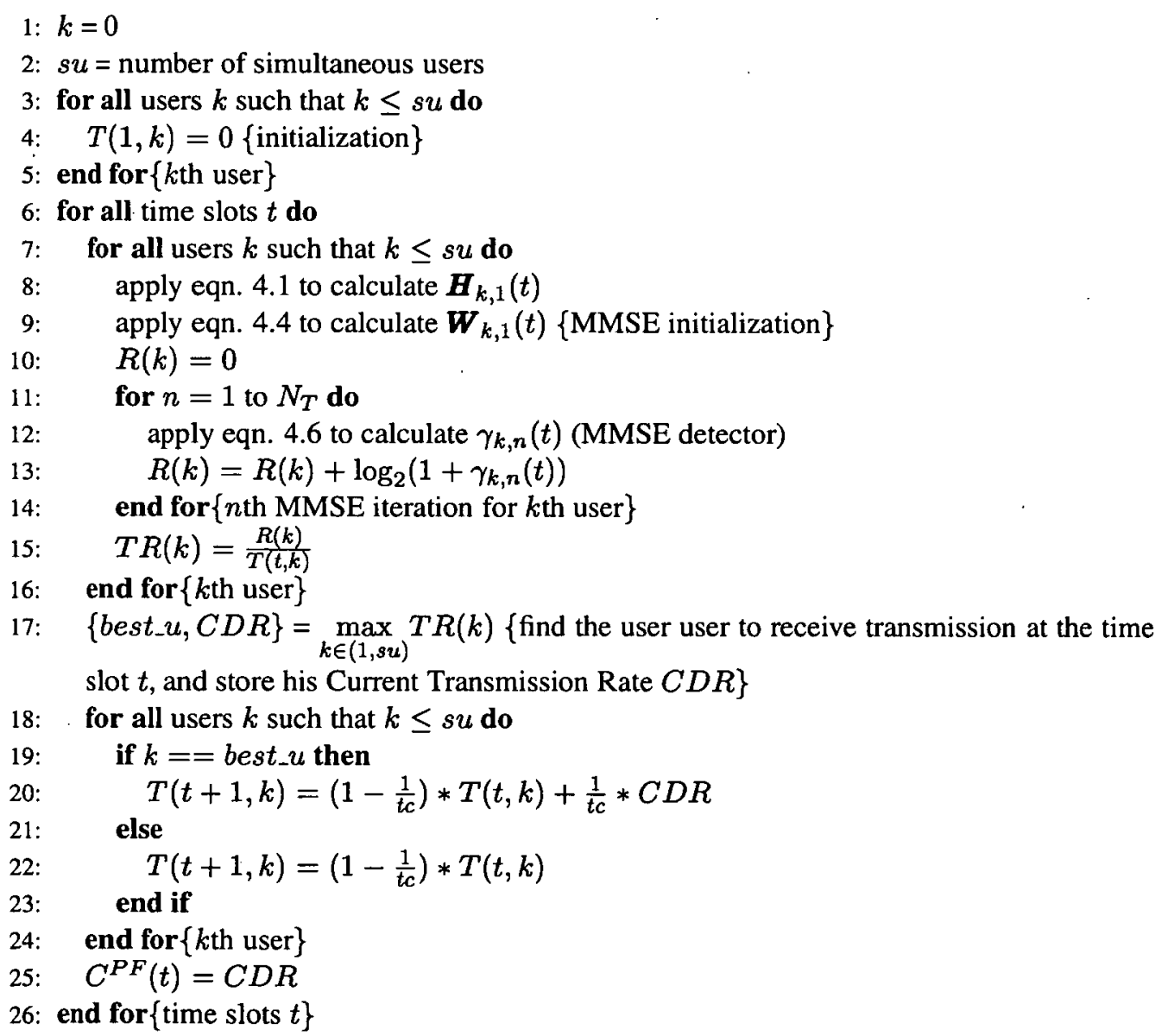

Figure B.1: $A A-P F M M S E$ single algorithm 
1: $k=0$

2: $s u=$ number of simultaneous users

3: for all users $k$ such that $k \leq s u$ do

4: $\quad$ for $n=1$ to $N_{T}$ do

5: $\quad T(1, k, n)=0$ initialization $\}$

6: end for $\{n$th antenna\}

7: end for $\{k$ th user $\}$

8: for all time slots $t$ do

9: $\quad$ for all users $k$ such that $k \leq s u$ do

10: $\quad\left\{\gamma_{k, 1}(t), \cdots, \gamma_{k, N_{T}}(t), D_{k, 1}(t), \cdots, D_{k, N_{T}}(t)\right\}=$ vblast_sinr $(k)\{$ vblast_sinr function (Figure (A.2)) calculates the SINR information after VBLAST detection $\}$

11: $\quad\left\{R(k, 1), \cdots, R\left(k, N_{T}\right)\right\}=\left\{\log _{2}\left(1+\gamma_{k, 1}(t)\right), \cdots, \log _{2}\left(\gamma_{k, N_{T}}(t)\right), D_{k, 1}(t), \cdots, D_{k, N_{T}}(t)\right\}$ end for $\{k$ th user $\}$

$T R(k, n)=\frac{R(k, n)}{T(t, k, n)}$

for $n=1$ to $N_{T}$ do

$\{$ best_u $(n), C D R(n)\}=\max _{k \in(1, s u)} T R(k, n)$ ffind the user user to receive transmission

at the time slot $t$ from antenna $n$, and store his Current Transmission Rate $C D R\}$

16: end for $\{n$th antenna $\}$

17: $S U G=$ best_u the elements of SUG are the users that achieve the highest data rates when assigned $\}$

18: $\quad D(t)=\left\{D_{S U G(1), 1}(t), \cdots, D_{S U G(1), N_{T}}(t), \cdots, D_{S U G\left(N_{T}\right), 1}(t), \cdots, D_{S U G\left(N_{T}\right), N_{T}}(t)\right\}$

19: $\quad$ for $k=1$ to $s u$ do

20: $\quad$ for $n=1$ to $N_{T}$ do

21: $\quad \boldsymbol{P}_{k n}(t)=k\left\{\boldsymbol{P}_{23}(t)=4=>\right.$ according to the 2nd mapping, the user 4 is assigned to antenna 3$\}$

end for $\{n$th antenna $\}$

end for $\{k$ th user $\}$

for $n=1$ to $s u$ do

$C^{A A-R R S-V B L A S T-P F}(n)=\quad$ Feasibility_Test $\left(n, N_{T}, P(t), D(t), \Gamma(t)\right)$

\{calculate the system capacity for the $n$th mapping sequence, the Feasibility $\operatorname{Test}()$

(Figure (A.3))function is discussed thoroughly in section 4.4.2\}

26: end for $\{n$th mapping sequence - exhaustive search

27: $\left\{\right.$ best_user, max_C $\left.C^{A A-R R S-V B L A S T-P F}\right\}=\max _{n \in\left(1, N_{T}\right)} C^{A A-R R S-V B L A S T-P F}(n)$

\{find the best user best_user that realizes the highest system capacity $\max \_C^{A A-R R S-V B L A S T-P F}=C^{A A-R R S-V B L A S T-P F}($ best_user $\left.)\right\}$

$R^{A A-R R S-V B L A S T-P F}=$ Feasibility_Test(best_mapping, $\left.N_{T}, P(t), D(t), \Gamma(t)\right)$

29: $\quad$ for $n=1$ to $N_{T}$ do

30: $\quad$ for all users $k$ such that $k \leq s u$ do

31: $\quad$ if $k==$ best_user then

$T(t+1, k, n)=\left(1-\frac{1}{t c}\right) * T(t, k, n)+\frac{1}{t c} * R^{A A-R R S-V B L A S T-P F}(n)$

else

$T(t+1, k, n)=\left(1-\frac{1}{t c}\right) * T(t, k, n)$

end if

end for $\{k$ th user $\}$

end for $\{n$th antenna $\}$

38: $\quad C^{A A-R R S-V B L A S T-P F}(t)=\max _{-} C^{A A-R R S-V B L A S T-P F}$

39: end for $\{$ time slots $t\}$

Figure B.2: $A A-P F-V B L A S T$ single algorithm 
1: $k=0$

2: $s u=$ number of simultaneous users

3: for all users $k$ such that $k \leq s u$ do

4: $\quad$ for $n=1$ to $N_{T}$ do

5: $\quad T(1, k, n)=0\{$ initialization $\}$

6: end for $\{n$th antenna $\}$

7: end for $\{k$ th user $\}$

8: for all time slots $t$ do

9: $\quad$ for all users $k$ such that $k \leq s u$ do

10: apply eqn; 4.1 to calculate $\boldsymbol{H}_{k, 1}(t)$

11: apply eqn. 4.4 to calculate $\boldsymbol{W}_{k, 1}(t)$ \{MMSE initialization\}

12: $\quad R(k, n)=0$

13: $\quad$ for $n=1$ to $N_{T}$ do

14:

15:

16:

17:

18 :

19:

20:

apply eqn. 4.6 to calculate $\gamma_{k, n}(t)$ (MMSE detector)

$R(k, n)=\log _{2}\left(1+\gamma_{k, n}(t)\right)$

$T R(k, n)=\frac{R(k, n)}{T(t, k, n)}$

end for $\{n$th MMSE iteration for $k$ th user $\}$

end for $\{k$ th user $\}$

for $n=1$ to $N_{T}$ do

$\{$ best_u $(n), C D R(n)\}=\max _{k \in(1, s u)} T R(k, n)\{$ find the user user to receive transmission at the time slot $t$ from antenna $n$, and store his Current Transmission Rate $C D R\}$

21: end for $\{n$th antenna $\}$

22: $\quad$ for $n=1$ to $N_{T}$ do

23: $\quad$ for all users $k$ such that $k \leq s u$ do

24:

25:

26:

27:

28:

29:

32: end for $\{$ time slots $t$ \}

if $k==$ best_ $_{-} u(n)$ then

$T(t+1, k, n)=\left(1-\frac{1}{t c}\right) * T(t, k, n)+\frac{1}{t c} * C D R(n)$

else

$T(t+1, k, n)=\left(1-\frac{1}{t_{c}}\right) * T(t, k, n)$

end if

end for $\{k$ th user $\}$

end for $\{n$th antenna $\}$

$C^{P F}(t)=\sum_{n=1}^{N_{T}} C D R(n)$

Figure B.3: $A A-P F-M M S E$ multi algorithm 


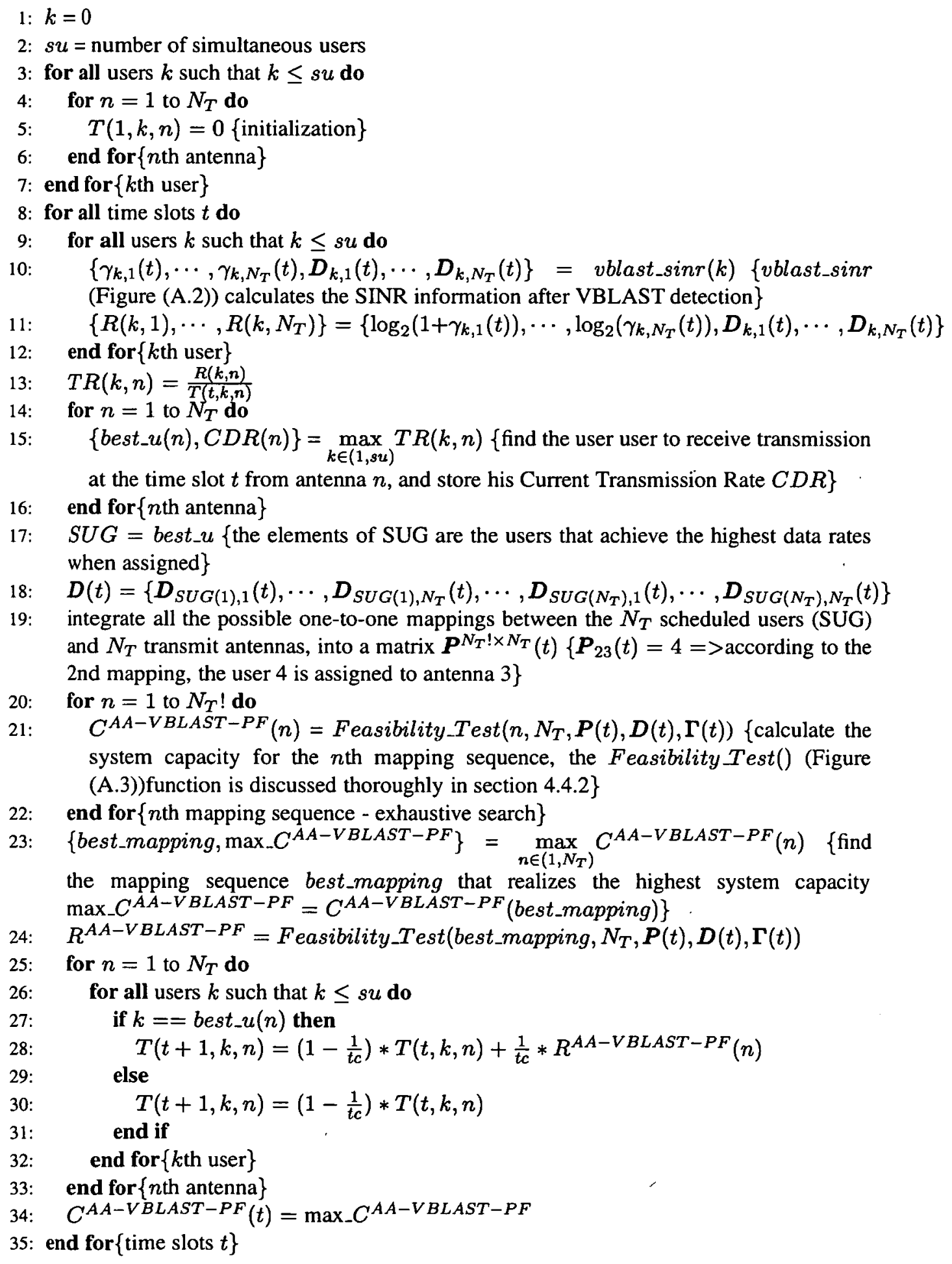

Figure B.4: $A A-P F-V B L A S T$ multi algorithm 


\section{Appendix C Publications}

- Constantine Floros and Steve McLaughlin and John S.Thompson, "Exploiting Multiuser Diversity for MIMO Cellular Systems using Packet Scheduling and the VBLAST Receiver ," 6th IEE International Conference on $3 G$ \& Beyond, London, vol.: 1, pp. 13-18, Nov. 2005.

- Constantine Floros and Steve McLaughlin and Loukianos Gatzoulis and John S.Thompson and Gordon Povey, "A Comparison of the MMSE Detector and its V-BLAST Version for a Stochastic MIMO Radio Channel Model," IEE DSP Enabled Radio Colloquium, ISLI Livingstone, Scotland, Sep. 2003 


\title{
EXPLOITING MULTIUSER DIVERSITY FOR MIMO CELLULAR SYSTEMS USING PACKET SCHEDULING AND THE VBLAST RECEIVER
}

\author{
Constantine Floros' \\ Steve MaLaughlin ${ }^{2}$ \\ John S.Thompsons \\ ${ }^{1}$ Institute for Digital Communications, School of Engineering and Electronics, The University of Edinburgh, \\ King's Buildings, Edinburgh EH9 3JL, UK. Konstantinos.Floros@ee.ed.ac.uk \\ Tel: +44 (0)131 6505655 Fax: +44 (0)1316506554 \\ ${ }^{2}$ Steve.McLaughlin@ee.ed.ac.uk, ${ }^{3} \mathrm{John} . T h o m p s o n @ e e . e d . a c . u k$
}

\begin{abstract}
Several packet scheduling techniques for the downlink of a multiple-input multiple-output (MIMO) cellular system are investigated. The system capacity for three scheduling algorithms, the conventional round robin scheduling (RRS), the antenna-assisted round robin (AA-RRS) and a novel VBLAST AA-RRS, are evaluated. Computer simulations are used to compare these scheduling schemes in terms of the expected and outage capacities. The novel scheme has proven to be beneficial for the system performance.
\end{abstract}

\section{INTRODUCTION}

Multiple-input multiple-output (MIMO) systems have been proven efficient for achieving high capacity over wireless links [1]. In MIMO systems based on spatial multiplexing each data stream is split into multiple substreams, and each of these substreams is transmitted through one of transmit antennas [2]. In multiuser environments, independence of fading among users, called multiuser diversity [3], can be exploited to increase the system capacity. Motivated by the information theoretic results in [4] and [5] one approach to increase the throughput of multiuser systems is to use multiuser diversity to take advantage of the independence of the fading statistics of the different users [6]. To benefit from multiuser diversity in cellular packet transmission systems, a packet scheduler should preferentially allocate radio resources to users in good channel conditions. Two essential goals of packet scheduling are to maximize the system capacity (data rate) and to provide fairness among users. In [3], a channel state dependent scheduling scheme maximizes the system capacity through the use of multiuser diversity. Specifically, each spatial channel is allocated to a user with the best channel condition for each time slot. Therefore, some users in adverse channel conditions may not be served, causing an unfairness problem. In [7], the round robin scheduling (RRS) scheme has been studied for MIMO cellular systems. The RRS scheme operates in a cyclic fashion regardless of the channel conditions, and thus achieves fairness among users [7]. The RRS however, does not use multiuser diversity, resulting in the same capacity as a single user system. In order to exploit the multiuser diversity and at the same time maintain fairness across the users, two other scheduling schemes have been proposed in [8] and [9]. In [9] the antenna-assisted round robin scheduling (AA-RRS) scheme has been proposed. The AA-RRS is an improved RRS scheme that exploits multiple antennas to achieve a diversity effect from multiple users. The opportunistic scheduling [8] uses multiple antennas and a proportional fair scheduler (PF). PF scheduler assigns a user for transmission when its instantaneous channel capacity is high relative to its average channel condition. As such, the benefit of multiuser diversity can be exploited and fairness can be maintained. This paper proposes a variation of AA-RRS scheme, where VBLAST detection is applied to each receiver. This novel technique is shown to improve significantly the system capacity.

\section{SYSTEM AND CHANNEL MODEL}

As depicted in Fig. 1, a base transceiver station (BTS) communicates with $K$ users. The BTS has $N_{T}$ transmit antennas while each user has $N_{R}$ receive antennas. A queue of packets is stored at the BTS for each of the $K$ users. The

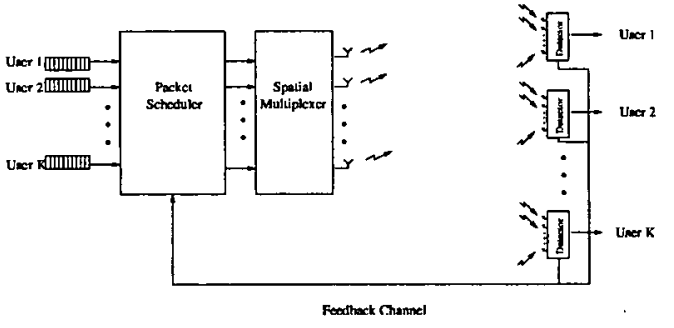

Figure 1. Downlink MIMO cellular system.

packet scheduler at the BTS determines which user's packet to transmit through each transmit antenna. The channel matrix $\boldsymbol{H}_{k}(t)$ between the BTS and the user $k$ for a time slot $t$ may be expressed as [9]

$$
\boldsymbol{H}_{k}(t)=\sqrt{S N R_{0}\left(r_{k} / R\right)^{-a} 10^{S_{k}(t) / 10}} G_{k}(t)
$$

where $S N R_{0}$ denotes the median SNR at the cell boundary, $R$ is the cell radius, $r_{k}$ is the distance between the BTS and the user $k, a$ is the path loss exponent, and $S_{k}(t)$ is the real Gaussian random variable with zero mean and variance of $\sigma_{S}^{2}$ that is used to model shadowing. An $N_{R} \times N_{T}$ matrix $G_{k}(t)$ represents Rayleigh-distributed multipath fading between the BTS and the user $k$. 


\section{RRS AND AA-RRS SCHEMES}

The RRS is described in [7]. The system capacity $C_{R R S}(t)$ may be expressed as

$$
C_{R R S}(t)=\sum_{n=1}^{N_{T}} \log _{2}\left(1+\gamma_{k, n}(t)\right)
$$

where $\gamma_{k, n}(t)$ denotes the post-detection SINR for the channel corresponding to the $n$th transmit antenna and the $k$ th user. The post-detection SINR is defined as the SINR of a transmit symbol after linear MMSE interference suppression, and it may be expressed as [9]

$$
\begin{aligned}
& \gamma_{k, n}(t)= \\
& \frac{\left.P_{R} \mid\left[W_{h}(t) B_{k}(t)\right]\right]\left._{n n}\right|^{2}}{\sigma_{N}^{2} N_{T} \sum_{m=1}^{N_{T}}\left|\left[W_{k}(t)\right]_{n m}\right|^{2}+P_{R} \sum_{m=1, m \neq n}^{N T}\left|\left[W_{h}(t) H_{k}(t)\right]_{n m}\right|^{2}}
\end{aligned}
$$

where $P_{R}$ is the total received signal power, and $\sigma_{N}^{2}$ is the noise power per received antenna. The nulling weight matrix $\boldsymbol{W}_{k}(t)$ for the MMSE detector is given as [9]

$$
W_{k}(t)=H_{k}^{H}(t)\left(H_{k}(t) H_{k}^{H}(t)+\left(\sigma^{2} N_{T} / P_{R}\right) I_{N_{R}}\right)^{-1}
$$

where $(\bullet)^{H}$ denotes the conjugate transpose, and $\boldsymbol{I}_{N_{R}}$ is the $N_{R} \times N_{T}$ identity matrix.

The AA-RRS scheme is described in [9]. At each time slot, $N_{T}$ users are selected from $K$ users in a round robin fashion, and these users form a scheduled user group (SUG). A mapping between transmit antennas and users may be represented as a sequence $\left(k_{1}, k_{2}, \ldots, k_{N_{T}}\right)$, with $k_{n}$ denoting the user index assigned to the $n$th transmit antenna. For a given sequence of $k_{n}$ 's the system capacity for time slot $t$ may be calculated as [9]

$$
\left.C_{A A-R R S}\left(t \mid\left(k_{1}, k_{2}, \ldots, k_{N_{T}}\right)\right)=\sum_{n=1}^{N_{T}} \log _{2}\left(1+\gamma_{k_{n}, n}\right)\right)
$$

\section{PROPOSED SCHEDULING SCHEMES}

In this section we introduce three scheduling algorithms. The AA-RRS Best User scheme (section 4.1), the VBLAST AA-RRS scheme (section 4.2) and the the VBLAST AARRS Best User scheme (section 4.4).

\subsection{AA-RRS Best User Scheme}

This scheme is based on the original AA-RRS scheme (section 3). According to the AA-RRS scheme, at each time slot, $N_{T}$ users are selected from $K$ users (in a round robin fashion), and the selected users form the scheduled user group (SUG). The proposed AA-RRS Best User scheme, selects the users to form the SUG in a different way. Specifically, each transmit antenna $n$ is assigned to the user $k$ that realizes the best spatial channel. This means that one user may be allocated to multiple transmit antennas unlike AARRS. The criterion to choose the best spatial channel, is the post-detection SINR information. The post-detection SINR between transmit antenna $n$ and user $k$ is given by the eqn. (3).

The obvious drawback of the proposed scheme is that the selection criterion for the SUG may introduce some level of unfairness between the users. Practically, the users with good spatial channels will be served more often than the users with poor spatial channels. In order to compensate for this unfairness, the users to be selected are chosen in a round robin fashion.

\subsection{VBLAST AA-RRS Feasible Data Rates Algo- rithm}

The propased VBLAST AA-RRS scheme is based on the AA-RRS scheme [9]. The basic difference is the use of VBLAST detectors at each receiver. VBLAST detection [2] is an improved technique for exploiting spatial multiplexing in MIMO multipath environment. The main idea of using VBLAST detection is the feature of the symbol cancellation. Using symbol cancellation, interference from already-detected components of the transmitted signal are subtracted out from the received signal vector, resulting in a modified receiver vector in which fewer interferers are present. When nulling with symbol cancellation are used together (VBLAST) the system performs better than in the case of pure nulling [2]. The post-detection $\operatorname{SINR}\left(\gamma_{k, n}(t)\right)$ for the channel corresponding to the nth transmit antenna and the $k t h$ user, is defined as the SINR of a transmit symbol after symbol cancellation and MMSE nulling, and it may be expressed as

$$
\begin{aligned}
& \gamma_{k, n}(t)= \\
& \frac{P_{R}\left|\left(W_{k}(t) H_{k}(t)\right]_{n n}\right|^{2}}{\sigma_{N}^{2} N_{T} \sum_{m=1}^{N_{T}}\left|\left[W_{k}(t)\right]_{n m}\right|^{2}+P_{R} \sum_{m=1, m \neq n, m \notin D_{h, n}(t)}^{N}\left|\left[W_{k}(t) H_{k}(t)\right]_{n m}\right|^{2}}
\end{aligned}
$$

where $D_{k, n}(t)$ denotes a set, whose elements are the transmit antennas that have been detected by user $k$ before the detection of transmit antenna $n$.

$$
D_{k, \mathrm{n}}(t)=\left\{d_{1,1}(t), d_{1,2}(t), \cdots, d_{1, \mathrm{n}_{T}}(t)\right\}
$$

where $d_{k, i}(t)$ denates the detected antenna from user $k$ after the ith iteration of the VBLAST detection algorithm. The nulling weight matrix $\boldsymbol{W}_{k}(t)$ for the MMSE is given as in eqn. (4). The modified receiver vector gives higher post-detection SINRs (eqn. (6)) and therefore higher system capacity.

To describe the algorithm analytically, consider $K$ active users that are initially listed in such a way that each user is identified with a unique user index $k(k=1,2, \ldots, K)$. At each time slot, $N_{T}$ users are selected from $K$ users, and these users form a scheduled user group (SUG) at the time slot. The selection of users for an SUG is made in a round robin fashion. For example, in the case of $K=6$ and $N_{T}=4$, the SUGs for subsequent time slots are $\{1,2,3,4\}$, $\{5,6,1,2\},\{3,4,5,6\}$, and so forth, where the numbers denote user indices. In the special cases of $K<N_{T}$, users are allowed to participate in the SUG more than once in a time slot. Once an SUG is formed, the spatial channels or transmit antennas should be assigned to users in the SUG. To guarantee fair channel access as in the RRS scheme, 
we restrict each scheduled user to use only one transmit antenna. This means that every user can use one spatial channel every $K / N_{T}$ time slots, or equivalently $N_{T}$ channels every $K$ time slots, as in RRS scheme. There are $N_{T}$ ! possible one-to-one mappings between $N_{T}$ scheduled users and $N_{T}$ transmit antennas, and an appropriate choice of mapping may realize a strong diversity effect. It is possible to integrate all the possible one-to-one mappings into a matrix $P(t)$ of size $N_{T} ! \times N_{T}$.

$$
\boldsymbol{P}(t)=\left[\begin{array}{cccc}
p_{1,2}(t) & p_{1,2}(t) & \cdots & p_{1, N_{T}}(t) \\
p_{2,1}(t) & p_{2,2}(t) & \cdots & p_{2, N_{T}}(t) \\
\vdots & \vdots & \ddots & \vdots \\
p_{N_{T} !, 1}(t) & p_{N_{T} !, 2}(t) & \cdots & p_{N_{T} !, N_{T}}
\end{array}\right]_{N_{T} ! \times N_{T}}
$$

where $p_{m, n}(t)$ denotes the user $k$ that is allocated to $n t h$ transmit antenna according to the $m t h$ mapping sequence. We utilize the post-detection SINR information for determining an effective mapping. The post-detection SINR is calculated at the receiver, and then is fed back from each user to the BTS, as in Fig. 1. The post-detection SINR information for the users in the SUG at time slot $t$ may be integrated into a matrix as

$$
\boldsymbol{\Gamma}(t)=\left[\begin{array}{cccc}
\gamma_{1,1}(t) & \gamma_{1,2}(t) & \cdots & \gamma_{1, N_{T}}(t) \\
\gamma_{2,1}(t) & \gamma_{2,2}(t) & \cdots & \gamma_{2, N_{T}}(t) \\
\vdots & \vdots & \ddots & \vdots \\
\gamma_{N_{T}, 1}(t) & \gamma_{N_{T}, 2}(t) & \cdots & \gamma_{N_{T}, N_{T}}
\end{array}\right]_{N_{T} \times N_{T}}
$$

where $\gamma_{k, n}(t)$ denotes the post-detection SINR for the channel between the $n t h$ transmit antenna and the $k t h$ user in the SUG, as expressed in (6). A mapping between transmit antennas and users may be represented as a sequence $\left(k_{1}, k_{2}, \ldots, k_{N_{T}}\right)$, with $k_{n}$ denoting the user index assigned to the $n t h$ transmit antenna. For a given sequence of $k_{n}$ 's the system capacity for the time slot $t$ may be calculated as

$\left.C_{A A-R R S-V B L A S T}\left(t \mid\left(k_{1}, k_{2}, \ldots, k_{N_{\gamma}}\right)\right)=\sum_{n=1}^{N_{T}} \log _{2}\left(1+\gamma_{k_{n}, n}\right)\right)$

where $\gamma_{k_{n}, n}$ denotes the post detection SINR for the channel the nth transmit antenna and the $k_{n}$ th user, as expressed in (6). The sequence of $k_{n}$ 's that maximizes the system capacity may be found using an exhaustive search (EXS) over all possible sequences.

\subsection{Feasible Data Rates}

The data rates of the new allocation scheme should be feasible. In short, the assumed data rates have to be smaller than the capacities of the wireless links. In the VBLAST algorithm, a signal must be detected successfully for it to be subtractively cancelled. This leads to additional capacity constraints as compared to the MMSE detector. If these constraints are not set, the VBLAST detectors would not be able to detect the users.

As an example, we consider the scenario of a single cell MIMO cellular system. The base station is equipped with a uniform linear array with 2 antenna elements and each mobile user is equipped with a uniform linear array with 2 antenna elements. After the application of the VBLAST method we have a "candidate" allocation that maximizes the overall system capacity. User 1 is assigned to the $1 \mathrm{~s}$ transmit antenna and user 2 is assigned to 2 nd transmit antenna. In order to accept this "candidaten allocation the assumed data rates, as derived from VBLAST detection, have to be smaller than the capacity of the wireless links. The VBLAST detection order may be integrated into a matrix as:

detection_order $=\left[\begin{array}{cccc}d t_{11} & d t_{12} & \cdots & d t_{1 N_{T}} \\ d t_{21} & d t_{22} & \cdots & d t_{2 N_{T}} \\ \vdots & \vdots & \ddots & \vdots \\ d t_{K 1} & d t_{K 2} & \cdots & d t_{K N_{T}}\end{array}\right]_{K \times N_{T}}$

where $d t_{k n}$ denotes the user, that is detected by user $k$ in $n$th position. $N_{T}$ is the number of transmit antennas and $K$ the number of users. In our scenario the detection matrix is:

$$
\text { detection_order }=\left[\begin{array}{ll}
1 & 2 \\
1 & 2
\end{array}\right]
$$

According to that matrix, the 1 st user has to be able to decode only the 1st signal, because the signal for the 1st user is received at first. That means that the data rate of the 1st signal has to be smaller or equal to the capacity of the wireless link connecting the BS to the 1st user:

$$
R_{1} \leq C_{1}
$$

If we apply eqn. (2) to the capacity of the wireless link of the 1st user we have :

$$
C_{1}=\log _{2}\left(1+\gamma_{1,1}\right)
$$

The 2nd user has to be able to decode the 1st and the 2nd signal. The 2nd signal is the signal for the 2nd user, but the 2nd user has to be able to decode the 1st signal first in order to be able to detect the 2nd signal. That means that the data rate of the 1st signal has to be smaller or equal to the capacity of the wireless link connecting the BS to the 2nd user. Thus, we have :

$$
R_{1} \leq C_{2}
$$

If we apply eqn. (2) to the capacity of the wireless link of the 2nd user we have :

$$
C_{2}=\log _{2}\left(1+\gamma_{2,1}\right)
$$

In order for eqns. (12) and (14) to be satisfied simultaneously we must have

$$
R_{1} \leq \log _{2}\left(1+\min \left(\gamma_{2,1}, \gamma_{1,1}\right)\right)=C_{1}
$$

The feasible system capacity is $C_{o v}=C 1+C 2=>$

$$
C_{o v}=\log _{2}\left(1+\min \left(\gamma_{2,1}, \gamma_{1,1}\right)\right)+\log _{2}\left(1+\gamma_{2,2}\right)
$$




\subsection{VBLAST AA-RRS Best User Scheme}

This scheme is based on the proposed VBLAST AA-RRS scheme (section 4.2). The VBLAST AA-RRS Best User scheme, selects the users to form the SUG in a different way. Specifically, each transmit antenna $n$ is assigned to the user $k$ that realizes the best spatial channel. The criterion to choose the best spatial channel, is the post-detection SINR information. The post-detection SINR between transmit antenna $n$ and user $k$ is given by the eqn. (3). As with AARRS Best User, this means that one user can be allocated to multiple transmit antennas.

Again the obvious drawback of the proposed scheme is that the selection criterion for the SUG may introduce some level of unfairness between the users. Practically, the users with good spatial channels will be served more often than the users with poor spatial channels.

\section{SIMULATION RESULTS}

The system capacities for the RRS, AA-RRS and the proposed scheduling schemes (AA-RRS best user, VBLAST AA-RRS, VBLAST best user) are evaluated and compared. The capacity for the RRS is calculated from eqn. (2) for 20,000 random realizations of the channel matrix in eqn. (1). To evaluate eqn. (2) for each channel matrix, the weight matrix of the receiver and the corresponding SINR values are computed using eqns. (4) and (3), respectively, under the assumption that the MMSE detection is employed. Similarly, the capacity for the AA-RRS scheme is calculated from eqn. (5). The capacity for the VBLAST AA-RRS scheme is calculated from eqn. (10), where the weight matrix of the receiver and the corresponding SINR values are computed using (4) and (6), respectively, under the assumption that MMSE detection is employed.

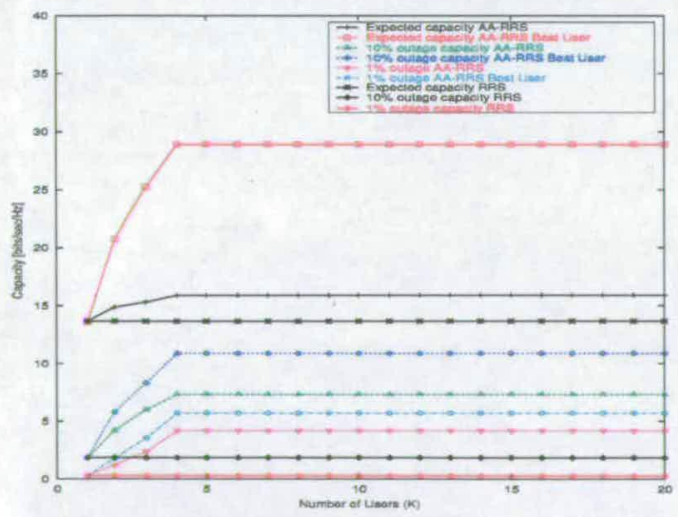

Figure 2. System capacities for a $(4,4)$ MIMO system.

The expected capacity is defined as the capacity averaged over all possible channel realizations, and the $x \%$ outage capacity is defined such that the probability of the capacity at a time slot being less than the value is $x \%$. The path loss exponent $a$ and log normal deviation of shadow fading $\sigma_{S}$ in eqn. (1) are assumed to be 3.7 and $8 \mathrm{~dB}$ respectively.
The channel is assumed to be spatially uncorrelated, i.e., $\boldsymbol{G}_{k}(t)=\boldsymbol{G}_{W, k}(t)$ in eqn. (1), where elements of $\boldsymbol{G}_{W, k}(t)$ are independent complex Gaussian random variables with zero mean and unit variance. We use $\left(N_{T}, N_{R}\right)$ to denote a MIMO system with $N_{T}$ transmit antennas and $N_{R}$ receive antennas.

\subsection{AA-RRS Best User vs AA-RRS}

Fig. 2 shows that the Best User scheme offers significant gain over the AA-RRS in terms of expected and outage system capacity. Specifically, for more than 4 users, the Best User scheme provides gains of: $84 \%$ in terms of expected capacity, $51 \%$ in terms of $10 \%$ outage capacity and $33 \%$ in terms of $1 \%$ outage capacity (the respective gains over RRS are: $110 \%, 590 \%$ and $1980 \%$ ). These results also indicate that the improvement of the Best User scheme is more substantial in terms of expected capacity, than in terms of the outage capacity. It should be noted that the outage capacity is a more important performance measure than the expected capacity for applications with delay constraint [10]. Another point to mention is that the system capacity for both schemes increses with the number of users $k$, until $k$ approaches the number of transmit antennas $N_{T}$. This is because both schemes obtain a diversity effect from different users in the SUG. This diversity effect is bounded by the number of different users in the same SUG, but the maximum number of users in the SUG is equal to the transmit antennas $N_{T}$. The above results stand for transmit power fixed to give $S N R_{0}=0 \mathrm{~dB}$.

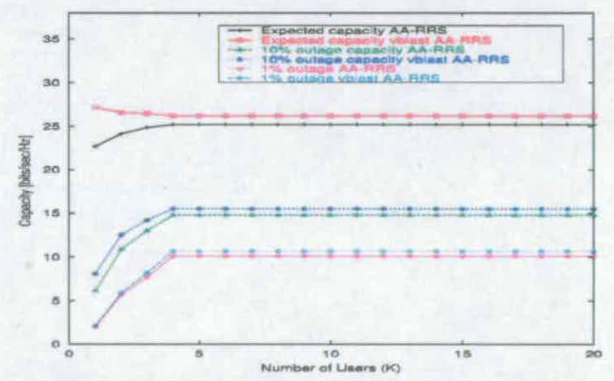

Figure 3. System Capacities for a $(4,4)$ MIMO system.

In Fig. 5 we investigate the fairness of the Best User algorithm. This graph depicts the average data rates of each user in the SUG of a $(4,4)$ MIMO system. It is shown that the AA-RRS scheme provides a higher level of fairness among users, than the Best User scheme. Namely, the AARRS scheduler shares the system capacity among all users of the SUG, which in the case of a $(4,4)$ MIMO system consists of 4 users. On the other hand, the Best User sceduler serves in practice only 2 users at each time slot.

\subsection{AA-RRS vs VBLAST AA-RRS}

As we can see from Fig. 3, the average system capacity of the proposed VBLAST AA-RRS scheduling scheme is increased about $5 \%$ over the conventional AA-RRS scheme. The results also indicate a similar improvement of the 
VBLAST AA-RRS scheme over the AA-RRS in terms of outage capacity. It should be noted that the system capacity of the VBLAST AA-RRS scheme does not increase with the number of users. That happens because the demand for feasible data rates restricts any possible capacity improvement that could occur due to multiuser diversity. The above results stand for transmit power fixed to give $S N R_{0}$ $=10 \mathrm{~dB}$.

\subsection{AA-RRS Best User vs VBLAST AA-RRS Best User}

In Figs. 4 and 5 the transmit power is fixed to give $S N R_{0}$ $=0 \mathrm{~dB}$. It can easily be observed from Fig. 4 that the VBLAST scheme offers significant gain over the AA-RRS in terms of expected and outage system capacity. Specifically, for more than 4 users, the VBLAST scheme provides gains of: $18 \%$ in terms of expected capacity, $28 \%$ in terms of $10 \%$ outage capacity and $22 \%$ in terms of $1 \%$ outage capacity.

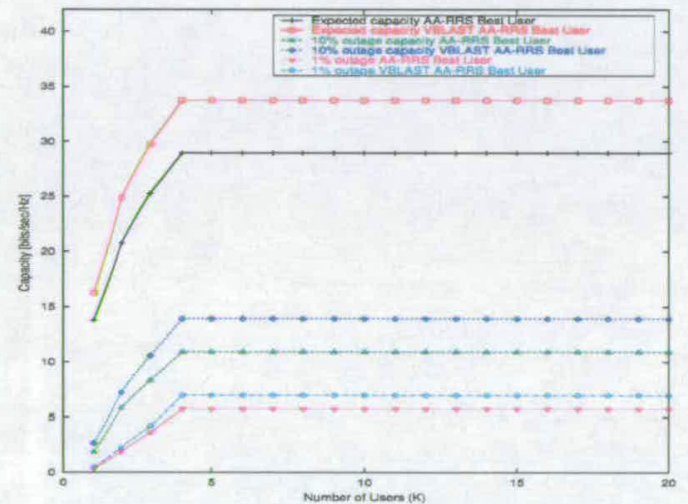

Figure 4. System Capacities for a $(4,4)$ MIMO system.

Finally, Fig. 5 shows that the conventional AA-RRS and the VBLAST AA-RRS schemes provide a higher level of fairness among users, than the Best User variants of these schemes. It should be noted that the Best User AA-RRS scheme is slightly less unfair than the Best User VBLAST AA-RRS scheme, which clearly favours one of the 2 users that are served each time slot.

\section{CONCLUSIONS}

In this paper, we proposed an efficient scheduler, an AARRS scheduling algorithm that employs the use of VBLAST detectors. The employment of VBLAST detectors at the receivers has been proven to be beneficial for the system performance. Two points worth mentioning here. First each user $k$ may need to decode data for another user, which may require the use of encryption for security reasons. Second, we do not consider error-propagation effects on VBLAST performance. Apart from the main work (VBLAST AARRS algorithm) we investigate the performance of the Best User AA-RRS and the Best User VBLAST AA-RRS algorithms. Both schemes are based on the AA-RRS and

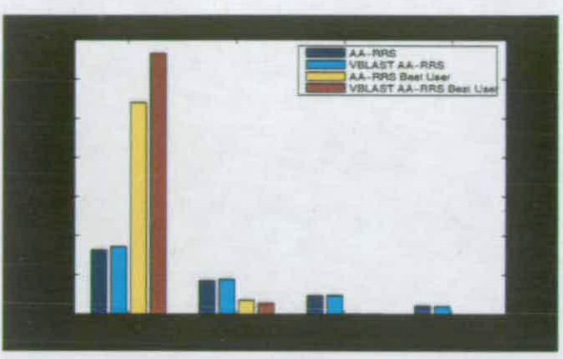

Figure 5. Data Rates for a $(4,4)$ MIMO system.

VBLAST AA-RRS schemes respectively. The basic difference is that the Best User schemes select to serve the users that realize the best spatial channels. The Best User schemes provide higher throughput but at the expense of fairness.

\section{REFERENCES}

[1] D.S. Shiu P.J. Smithi A. Naguib D. Gesbert, M. Shafi. From theory to practice: An overview of MIMO space-time coded wireless systems. IEEE Journal on Selected Areas in Communications., 21(3):281-302, April 2003.

[2] G.D. Golden et al. Detection algorithm and initial laboratory results using $V B L A S T$ spacetime communication architecture. Electron. Lett., 35(1):14-16, January 71999.

[3] M. Airy R.W. Heath and A.J. Paulraj. Multiuser diversity for MIMO wireless systems with linear receivers. Proc, Asilomar Conf. Signals, Systems, and Computers, Pacific Grove, CA, pages 1194-1199, Nov. 2001.

[4] R. Knopp and P. Humblet. Information capacity and power control in single cell multiuser communications. in Proc. of Int. Conf. on Comm. 1995, June 1995.

[5] D.N.C. Tse. Optimal power allocation over parallel gaussian channels. in Proc. of ISIT, page 27, 1997.

[6] D.N.C. Tse. Multiuser diversity n wireless networks. Wireless Communications Seminar, Stanford University, April 2001.

[7] Lucent Technologies. Throughput simulations for MIMO and transmit diversity enhancements to HSDPA. $3 G P P$ TSG RAN WG1.

[8] D.N.C. Tse P. Viswanath and R. Laroia. Opportunistic beamforming using dumb antennas. IEEE Trans. Inform. Theory, 48(6):1277-1294, Jun. 2002.

[9] Oh-Soon Shin and Kwang Bok(ED) Lee. Packet scheduling for MIMO cellular systems. IEEE Vehicular Technology Conference, pages 1694-1698, Apr. 2003.

[10] D. Paulraj A.J Bolcskei, H. Gesbert. On the capacity of ofdm-based spatial multiplexing systems. Communications, IEEE Transactions on, 50:225-234, Feb 2002. 


\title{
A COMPARISON OF THE MMSE DETECTOR AND ITS V-BLAST VERSION FOR A STOCHASTIC MIMO RADIO CHANNEL MODEL
}

\author{
Constantine Floros ${ }^{1} \quad$ Steve McLaughlin ${ }^{1} \quad$ Loukianos Gatsoulis ${ }^{2} \quad$ John S.Thompson ${ }^{1} \quad$ Gordon Povey \\ This work was supported by Elektrobit (UK) Ltd. \\ ${ }^{1}$ Institute for Digital Communications, School of Engineering and Electronics, The University of Edinburgh, \\ King's Buildings, Edinburgh EH9 3JL, UK. Konstantinos.Floros Qee.ed.ac,uk \\ Tel: +44 (0)131 6505655 Fax: $+44(0) 1316506554$ \\ ${ }^{2}$ Elektrobit (UK) Ltd, Technology Transfer Centre, Mayfield Road, Edinburgh EH9 3 JL \\ Tel: +44(0)131 4724701 Fax: +44 (0)131 6624678 WWW: www.elektrobit.co.uk
}

\section{ABSTRACT}

1 Theoretical and experimental studies of multiple-input multiple-output (MIMO) radio channels are presented in this paper. We investigate the performance of detection algorithms for single user wireless communication using multiple antennas at both the transmitter and receiver. The algorithms are based on the MMSE detector and its BLAST versions. Comparisons are made using bit error ratio (BER) versus signal-to-noise ratio (SNR) simulations. The system includes $M$ transmitting antennas and $N$ receiving antennas $M \leq N$. The effects of error propagation on algorithm performance are investigated for BPSK and 16-QAM modulation schemes. A simple stochastic MIMO model channel has been used. This model uses the correlation matrices at the mobile station (MS) and the (BS) so that results of numerous single-input multiple output (SIMO) studies that have been published in the literature can be used as input parameters. In this paper, the model is simpified to consider narrowband channels.

\section{INTRODUCTION}

In recent years the application of antenna arrays for wireless cellular systems has received much attention. Exploiting antenna arrays at both the transmitter and receiver allows the Shannon capacity of wireless channels to be increased significantly [1]. Multiple-in put multiple-output (MIMO) systems promise large theoretical information capacities, enabling high data rate transmission, especially in rich multipath indoor scenarios. They are candidates for very broadband wireless local area networks. However, to develop such systems, it is essential to have an accurate description of the underlying radio channel. There exists already a manifold of different stochastic or geometricallybased stochastic MIMO models [2] [3] [4] [5].

Large capacity is obtained via the potential decorrelation in the MIMO radio channel, which can be exploited to create many parallel subchannels. However the potential capacity gain is highly dependent on the multipath richness in the radio channel, since a fully correlated MIMO channel only offers one subchannel, while a completely decorrelated channel offers multiple subchannels depending on the antenna configuration. Today, most studies have been conducted assuming either fully correlated/decorrelated chan-

\footnotetext{
${ }^{1}$ This work was supported by Elektrobit (UK) Ltd.
}

nels [2] [6], while a partially correlated channel should be expected in practice. The objective of this paper is to investigate the performance of a simple MIMO radio channel model, which is applicable for link level simulations, using some popular detection algorithms (V-BLAST, MMSE). Some authors ([7]) have approached the problem from a geometrically-based perspective. A mathematical framework for a simple stochastic wideband MIMO channel was presented in [4]. In this paper we use a simplified model first presented in [8]. One of the main strengths of the MIMO stochastic model is that it relies on a small set of parameters to fully characterize the communication scenario; the power gain of the MIMO channel matrix, two correlation matrices describing the correlation properties at both ends of the transmission links, and the associated Doppler spectrum of the channel paths. The system model assumes $M$ transmitting antennas and $N$ receiving antennas $M \leq N$. Simulations are carried out for two modulation schemes (BPSK and 16-QAM). Multistream detection is implemented at the receiver. The minimum mean squared error detector (MMSE) is compared with its V-BLAST version. Comparisons are made using bit error ratio (BER) versus signal-tonoise ratio (SNR) simulation. The effect of error propagation in the BLAST (Bell Laboratories Layered Space-Time) scheme is also investigated.

\section{SYSTEM DESCRIPTION}

Consider the MIMO setup pictured in Fig. 1 with $M$ antennas at the transmitter and $N$ antennas at the receiver. A single data stream is demultiplexed into $M$ substreams, and each substream is then encoded into symbols and fed to its respective transmitter.

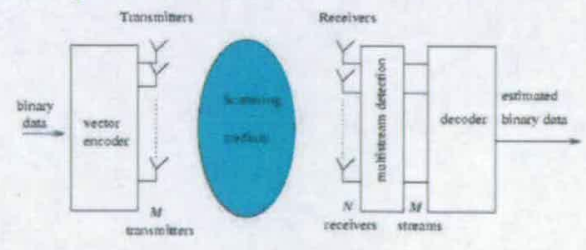

Figure 1. The $(\mathrm{M}, N)$ single user wireless communication system

Let $T$ seconds be the symbol period. Transmitter antennas 1 to $M$ operate on the same radio frequency at a symbol rate $1 / \mathrm{T}$ symbols/sec, with synchronized symbol timing. The collection of transmitters comprises, in effect, a 
vector-valued transmitter, where components of each transmitted $M$-vector are symbols drawn from a BPSK or QAM constellation. In this paper the same constellation is used for each substream, and transmissions are organized into a burst of $J$ symbols. The power launched by each transmitter is proportional to $1 / M$ so that the total radiated power is constant and independent of $M$. In V-BLAST the vector encoding process is simply a demultiplex operation followed by independent bit-to-symbol mapping of each substream. For the remainder of the paper, we will assume for simplicity that the substreams comprise uncoded, independent data symbols. Receivers 1 to $N$ are, individually, conventional BPSK or QAM receivers. These receivers also operate on the same radio frequency, each receiving the signals radiated from all $M$ transmit antennas. In this paper each symbol to be transmitted is sent to exactly one transmitting antenna, the receiver is symbol-synchronous. Letting $a$ $=\left(a_{1}, a_{2}, \ldots, a_{M}\right)^{T}$ denote the vector of transmitt symbols, then the corresponding received $N$-vector is

$$
\boldsymbol{r}_{1}=\boldsymbol{H a}+\boldsymbol{v}
$$

where $v$ is a noise vector with components drawn from IID wide-sense stationary zero mean processes with variance $\sigma^{2} . H$ is the $N \times M$ matrix channel transfer function, where $h_{i j}$ is the (complex) transfer function from transmitter $\mathrm{j}$ to receiver $i$, and $M \leq N$. We take the quasi-stationary viewpoint that the channel time variation is negligible over the $J$ symbol periods comprising a burst, and that the channel is estimated accurately, e.g. by use of a training sequence embedded in each burst. Thus, for brevity we will not make the distinction between $H$ and its estimate.

\section{DETECTION ALGORITHMS}

3.1. Minimum Mean Squared Error Detector (MMSE)

MMSE detection [9] [10] is represented by an $N \times M$ matrix $C$ which minimises :

$$
\sum_{k=0}^{M} E\left[\left\|(C r-a)_{k}\right\|^{2}\right]
$$

If $\sigma^{2}>0$ then MMSE may be represented by

$$
C_{M M S E}=\sqrt{P} H^{H}\left[P H H^{H}+\frac{\sigma^{2}}{p}\right]^{-1}
$$

In this paper $\rho=1$ for BPSK and $\rho=4$ for 16-QAM. $P$ is the total transmitted power.

\subsection{VBLAST detection algorithm}

In this section VBLAST detection algorithm is described ([11]). Let the ordered set

$$
S=\left\{k_{1}, k_{2}, \ldots, k_{M}\right\}
$$

be a permutation of the integers $1,2, \ldots, M$ specifying the order in which components of the transmitted symbol vector $\boldsymbol{a}$ are extracted, the detection algorithm operates on $\boldsymbol{r}_{1}$, progressively computing decision statistics $y_{k_{1}}, y_{k_{2}}, \ldots, y_{k_{M}}$, which are then sliced to form estimates of the underlying data symbols $\hat{a}_{k_{1}}, \hat{a}_{k_{2}}, \ldots, \hat{a}_{k_{M}}$. Thus, decision statistic $y_{k_{1}}$ is computed first then $y_{k_{2}}$, and so on. To determine a particular ordering $S_{\text {copt }}$ which is optimal in a certain sense we apply an optimum criterion (presented later). For now we assume an arbitrary ordering $S$. The detection process uses linear combinational nulling and symbol cancellation to successively compute the $y_{k_{2}}$, proceeding generally as follows:

Step 1: Using nulling vector $w_{k_{1}}$, form a linear combination of the components of $\boldsymbol{r}_{1}$ to yield $y_{k_{1}}$ :

$$
y_{k_{1}}=w_{k_{1}}^{T} r_{1}
$$

Step 2: Slice $y_{k_{1}}$ to obtain $\hat{a}_{k_{1}}$

$$
\hat{a}_{k_{3}}=Q\left(y_{k_{1}}\right)
$$

where $Q($.$) denotes the quantisation (slicing) operation$ appropriate to the constellation in nse.

Step 3: Assuming that $\hat{a}_{k_{1}}=a_{k_{1}}$, cancel $a_{k_{1}}$ from the received vector $r_{1}$, resulting in a modified received vector $r_{2}$ :

$$
r_{2}=r_{1}-\hat{a}_{k_{1}}(H)_{k_{1}}
$$

where $(\boldsymbol{H})_{k_{1}}$ denotes the $k_{1}$ th column of $\boldsymbol{H}$. Steps $1-3$ are then performed for components $k_{2}, \ldots, k_{M}$ by operating in turn on the progression of modified received vectors $r_{2}$, $r_{3}, \ldots, r_{M}$.

The most common choices for the criterion chosen to compute the nulling vectors $w_{k_{i}}$ are mean-squared error (MMSE) and zero-forcing (ZF). Both of these criteria are described and implemented here.

The $Z F$ criterion: The $k_{i}$ th $Z \mathrm{~F}$-nulling vector is defined as the unique minimum norm vector satisfying

$$
w_{k_{i}}{ }^{T}(H)_{k_{j}}= \begin{cases}0 & j>i \\ 1 & j=i\end{cases}
$$

Thus, the $k_{i}$ th ZF-nulling vector is orthogonal to the subspace spanned by the contributions to $r_{k_{i}}$ due to those symbols not yet estimated and canceled. It is not difficult to show that the unique vector satisfying eqn. 8 is just the $k_{i}$ th row of $\boldsymbol{H}_{k_{1-1}}^{+}$where the notation $\boldsymbol{H}_{\overline{k_{i}}}$ denotes the matrix obtained by zeroing columns $k_{1}, k_{2}, \ldots, k_{i}$ of $H$ and ${ }^{+}$denotes the Moore-pseudoinverse [12].

The MMSE criterion: VMMSE differs from the VBLAST-ZF in that the nulling vector derives from the solution of the eqn. (2), which is actually defined in eqn. (3).

\subsection{Why VBLAST?}

V-BLAST realizes higher spectral efficiencies than conventional multiple-access techniques(CDMA, FDMA, TDMA). In fact, an essential feature of BLAST is that no explicit orthogonalization of the transmitted signals is imposed by the transmit structure. Instead, the propagation environment itself, which is assumed to exploit significant multipath, is used to achieve the signal decorrelation necessary to separate the co-channel signals. Unlike code-division or other spread-spectrum multiple access techniques, the total channel bandwidth utilised in a BLAST system is only a small fraction in excess of the symbol rate. Unlike FDMA, each transmitted signal occupies the entire system bandwidth. 
Finally, unlike TDMA, the entire system bandwidth is used simultaneously by all of the transmitters all of the time.

\section{STOCHASTIC MIMO CHANNEL MODEL}

Detailed description of the stochastic radio channel adopted in this paper can be found in [4] and [8].

Let assume that the transmitter is the BS and the receiver is the MS. All antenna elements in the two arrays have the same polarization and the same radiation pattern. The spatial complex correlation coefficient at the BS between antenna $m_{1}$ and $m_{2}$ is given by

$$
\rho_{m_{1} m_{2}}^{B S}=\left\langle h_{m_{1} n}, h_{m_{2} n}\right\rangle
$$

where $\langle a, b\rangle$ compntes the correlation coefficient between $a$ and $b$. From (9) it is assumed that the spatial correlation coefficient at the BS is independent of $n$, since the $n$ elements at the MS, illuminate the same surrounding scatterers and, therefore, also generate the same power azimuth spectrum (PAS) as the BS. The Spatial correlation function is the Fourier transform of the PAS [13]. Different expressions of the spatial correlation function have been derived in the literature assuming that the PAS follows some specific functions ([14] [15]). The spatial complex correlation coefficient observed at the MS is similarly defined as

$$
\rho_{n_{1} n_{2}}^{M S}=\left\langle h_{m n_{1}}, h_{m n_{3}}>\right.
$$

and assumed to be independent of $\mathrm{m}$.

Given the (9) and (10), the following symmetrical complex correlation matrices can be defined

$$
R_{B S}=\left[\begin{array}{cccc}
\rho_{11}^{B S} & \rho_{12}^{B S} & \cdots & \rho_{1 M}^{B S} \\
\rho_{21}^{B S} & \rho_{22}^{B S} & \cdots & \rho_{2 M}^{B S} \\
\vdots & \vdots & \ddots & \vdots \\
\rho_{M S}^{B S} & \rho_{M S}^{B S} & \cdots & \rho_{M M}^{B S}
\end{array}\right]_{M \times M}
$$

and

$$
R_{M s}=\left[\begin{array}{cccc}
\rho_{11}^{M S} & \rho_{12}^{M S} & \cdots & \rho_{1 N}^{M S} \\
\rho_{21}^{M S} & \rho_{22}^{M S} & \cdots & \rho_{2 N}^{N S} \\
\vdots & \vdots & \ddots & \vdots \\
\rho_{N 1}^{M S} & \rho_{N 2}^{M S} & \cdots & \rho_{N N}^{N S}
\end{array}\right]_{N \pm N}
$$

The correlation coefficient between two arbitrary transmission coefficients connecting two different sets of antennas is expressed as

$$
\rho_{n_{2} m_{2}}^{n_{1} m_{1}}=\left\langle h_{m_{1} n_{1}, h_{m_{2} n_{2}}}>\right.
$$

which is equivalent (proof presented in [8]) to

$$
\rho_{n_{2} m_{2}}^{n_{1} m_{1}}=\rho_{n_{2} n_{2}}^{M S} \rho_{m_{1} m_{2}}^{B S}
$$

this means that the spatial correlation matrix of the MIMO radio channel is the Kronecker product of the spatial correlation matrix at the MS and the BS and is given by

$$
R_{M I M O}=R_{M S} \otimes R_{B S}
$$

\section{GENERATION OF SIMULATED CORRELATED CHANNEL COEFFICIENTS}

Correlated channel coefficients $h_{m r}$ are generated from zero-mean complex independent identically distributed (i.i.d.) random variables $d_{m n}$ shaped by the desired Doppler spectrum such that

$$
H=C d
$$

where $\boldsymbol{H}_{M N \times 1}=\left[h_{11}, h_{21}, \ldots, h_{M 1}, h_{12}, \ldots, h_{M N}\right]^{T}$ and $\boldsymbol{d}_{M N x 1}=\left[d_{1}, d_{2}, \ldots, d_{M N}\right]^{T}$ where the symetrical mapping matrix $C$ results from the standard Cholesky factorization of the matrix $\boldsymbol{R}_{\text {MIMO }}=\boldsymbol{C} \boldsymbol{C}^{T}$ provided that $\boldsymbol{R}_{\text {MTMO }}$ is nonsingular [12]. The generation of the simulated MIMO chanuel matrix $\hat{\boldsymbol{H}}$ can be deduced from the vector $\boldsymbol{H}$. Note that the correlation matrices and the Doppler spectrum cannot be chosen independently, as they are connected through the PAS at the MS [16].

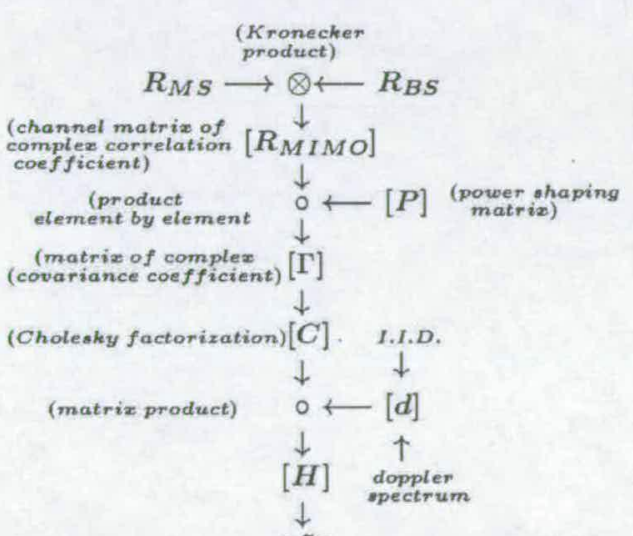

$[\tilde{H}]$

Figure 2. Flow chart illustrating the practical procedure to obtain correlated channel coefficient.

The flow chart illustrating the complete procedure to generate correlated channel coefficient is is presented in Fig. 2. The power-shaping matrix $\boldsymbol{P}$ represents any imbalance in BPR (Branch Power Ratio) between antenna elements. The elements of $\boldsymbol{P}$ are the product of the standard deviations $\sigma_{h_{m n}}$ of the channel coefficient $h_{m n}$

$$
P=\left[\begin{array}{cccc}
\sigma_{h_{11}}^{2} & \sigma_{h_{11}} \sigma_{h_{12}} & \cdots & \sigma_{h_{11}} \sigma_{h_{M M}} \\
\sigma_{h_{12}} \sigma_{h_{11}} & \sigma_{h_{13}}^{2} & & \\
\ldots & & \ddots & \\
\sigma_{h_{12}} \sigma_{h_{N N}} & & & \sigma_{h_{N M}}^{2}
\end{array}\right]
$$

Matrix $\mathrm{\Gamma}$ results from the element-by-element product of $\boldsymbol{R}_{\text {MIMO }}$ by matrix $\boldsymbol{P}$. Applying the Gaxpy algorithm ([12] 
p.143) (Cholesky decomposition) to $\Gamma$ we get the lowertriangular matrix $C$. Let $d$ be a vector of $M N$ complex zero-mean unit variance independent random variables. As described in eqn. (15), correlated channel coefficients $h_{m n}$ are generated from the $M \times N$-vector $\boldsymbol{d}$ and the matrix $C$

\section{SIMULATION}

Bit error ratio (BER) versus signal-to-noise ratio (SNR) simulations were carried out in order to compare the performance of the MMSE and VMMSE for different degree of correlation in the radio channel. Two types of modulation have been nsed : BPSK and 16-QAM. The effect of error propagation (EP) in the V-BLAST is also investigated. The curves labeled NEP (no error propagation) were obtained by implementing perfect cancellation by substraction. Signalto-noise ratios between $-10 \mathrm{~dB}$ and $20 \mathrm{~dB}$ were considered, were SNR of $v \mathrm{~dB}$ corresponds to $\frac{p}{a^{2}}=10^{\frac{1}{10}}$. For all the simulations, each frame was of length 100. Each frame corresponds to one simulated channel and $100 \mathrm{M}$ transmitted symbols. Each point plotted, is the average of 10000 simulated channels.

\subsection{Simulation Setup}

To introduce correlation into the simulation scenarios we have used the MIMO theoretical radio channel that results after the application of the algorithm described previously. In order to validate the stochastic MIMO model we have used channel correlation figures resulting from measurements [8]. The input parameters used in the validation stage are the average spatial complex correlation matrices $R_{B S}$ and $R_{M S}$. The measured spatial complex correlation matrices are the results of an average over the reference transmitting and receiving antennas. Two real examples have been used as input.

Example 1: Picocell Decorrelated. See (16.)

Example 2: Microcell Correlated. See (17.)

Both in examples 1 and 2, $\boldsymbol{R}_{M S}$ is decorrelated. This is expected since the MS is surrounded by scatterers. On the other hand, $\boldsymbol{R}_{B S}$ presents two different behaviors. In Example 1 , the spatial correlation coefficient remain low as expected in the case of an indoor termination. On the other hand, the spatial correlation coefficients at the BS are highly correlated in Example 2 with a mean absolute value of the coefficient of 0.96 . The high correlation is explained by the fact that in this specific example the BS is located above any surrounding scatterer. Therefore, a low azimuth spread (AS) is expected, which causes the antenna array elements to be highly correlated.

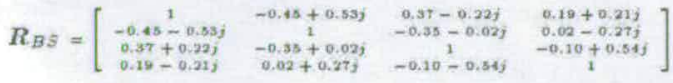

$$
\begin{aligned}
& R_{M S}=\left[\begin{array}{cccc}
2 & -0.13-0.62 j & -0.49+0.23 j & 0.15+0.28 j \\
-0.13^{2}+0.62 j & -0.13+0.52 j & -0.13-0.52 j & -0.58+0.12 j \\
-0.49-0.23 j & -0.13+0.15 & 0.02-0.62 j \\
0.15-0.28 j & -0.36-0.12 j & 0.02+0.61 j & 1
\end{array}\right] \\
& \boldsymbol{R}_{B S}=\left[\begin{array}{cccc}
1 & -0.61+0.77 j & 0.14-0.24 j & 0.24+0.49 j \\
-0.61-0.77 j & 1 & -0.85+0.30 j & 0.57-0.76 j \\
0.14+0.94 j & -0.85-0.50 j & 1 & -0.01+0.40 j \\
0.24-0.80 j & 0.57+0.78 j & -0.91-0.40 j & 1
\end{array}\right]
\end{aligned}
$$

$R_{M S}=\left[\begin{array}{cccc}-0.12+0.18 j & -0.12-0.16 j & 0.00+0.05 j & -0.02-0.13 j \\ 0.00-0.05 j j & -0.17+0.16 j & -0.17-0.16 j & 0.11+0.04 j \\ -0.02+0.13 j & 0.11-0.04 j & -0.27+0.16 j & -0.17-0.16 j\end{array}\right]$

6.2. Simulation results

For uncorrelated chamels and 16-QAM modulation, both detectors give essentially the same performance over the entire range of SNRs, In Fig. 3 the BERs (Bit Error Ratio) versus SNR for two detectors (VMMSE/MMSE) and BPSK modulation are plotted. At lower SNRs, both detectors (assuming EP in VBLAST MMSE) give rather similar performances. As SNR increases, the BER versus SNR curves diverge. At higher SNR, despite taking EP (Error Propagation) into account, the VMMSE detector still performs better than MMSE. From the same graph we can see the superiority of the MIMO $4 \times 4$ system over the SISO system regarding the BER performance.

In Fig. 4 the effect of EP, in the performance of VMMSE detector for BPSK and 16-QAM modulations for uncorrelated channels, is investigated. Note that 16-QAM modulation suffer more from EP than BPSK modulation.

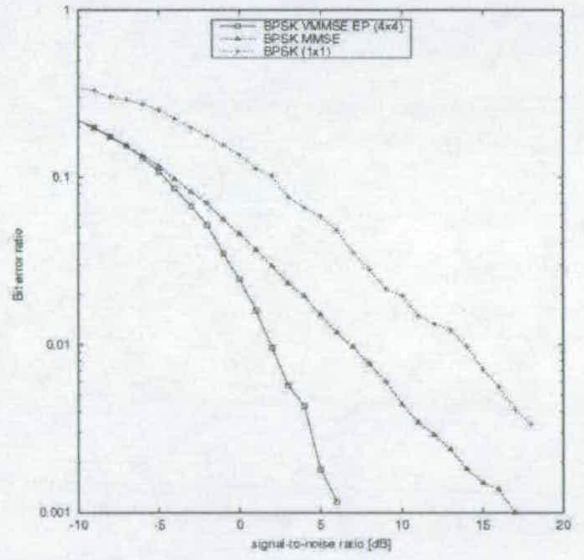

Figure 3. Detector comparison, $\mathrm{N}=4, \mathrm{M}=4$, BPSK modulation

In Fig. 5 we investigate the effect of correlation in the performance of VMMSE detector for BPSK and 16-QAM modulations. The presense of correlation can change dramatically the behavior of the detector. Similar results hold for MMSE detector as well (Fig. 6). This is due to the fact that correlation between the antenna array elements results in a channel matrix with low rank [1]. The channel $H$ may offer $K$ parallel subchannels with different mean gains, with $K=\operatorname{Rank}(R \leq \min (\mathrm{M}, \mathrm{N}))$, where the function Rank(.) and $\min ($.$) return the rank of the matrix ant$ minimum value of the arguments, respectively. $R$ is the instantaneous correlation matrix $\boldsymbol{R}=\boldsymbol{H} \boldsymbol{H}^{\boldsymbol{H}}$. When the rank $K$ of the channel matrix is low(high correlation), then 
less subchannels are available to the system, which results in degradation of the performance of the system.

The notation 'Corr exmpl' stands for the correlated example (see (17)) and the notation 'decorr exmpl' stands for the decorrelated example (see (16)). For BPSK modulation the performance of the VMMSE detector is much better than for 16-QAM over the entire range of SNR and specially for higher SNRs. From the comparison of MMSE and VMMSE detectors (Fig. 6), over the entire range of correlation, we can see that for lower SNRs the performance of both detectors is similar. But for higher SNRs the VMMSE detector performs better no matter the degree of correlation.

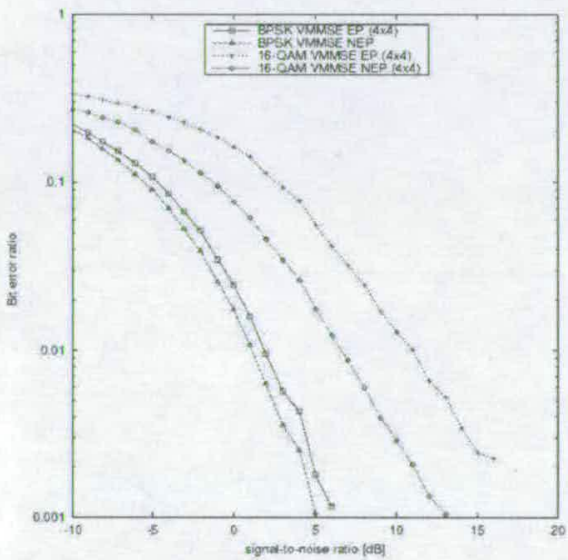

Figure 4. The effect of error propagation, $\mathrm{N}=4, \mathrm{M}=4$, BPSK/16QAM modulation

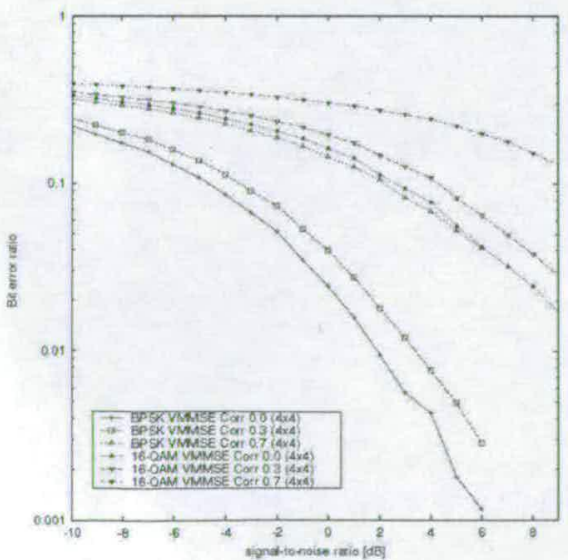

Figure 5. The effect of correlation, $N=4, M=4, B P S K / 16-Q A M$ modulation
Fig. 7 presents the impact of correlation on VMMSE performance for BPSK modulation. The graph confirms that the correlation between the antenna array elements degrades the performance of the system [1], [17].

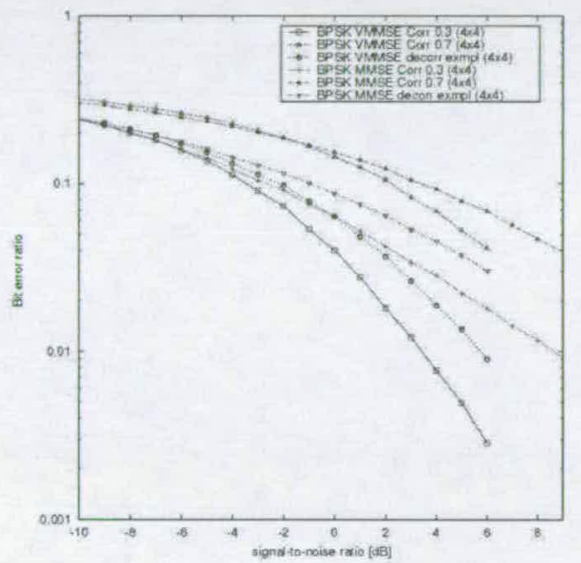

Figure 6 . The effect of correlation, $\mathrm{N}=4, \mathrm{M}=4$, BPSK modulation, detectors MMSE/VMMSE

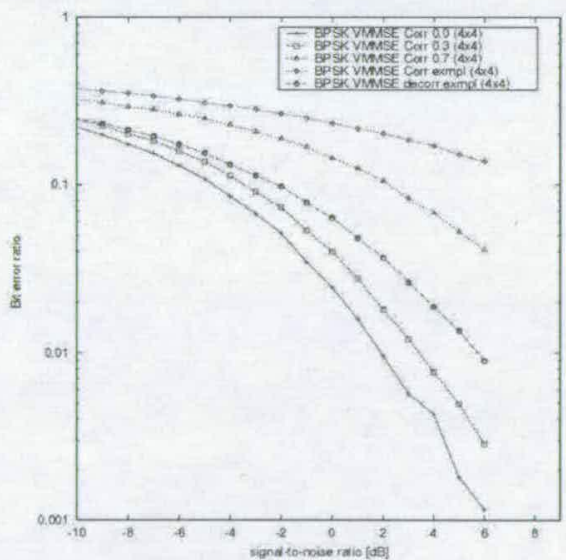

Figure 7. The effect of correlation, $\mathrm{N}=4, \mathrm{M}=4$, BPSK modulation, detector VMMSE

\section{CONCLUSIONS}

Detection algorithms for single-user wireless communication using multiple antennas at both the transmitter and receiver in a stochastic MIMO radio channel model are compared for different degree of correlation between the antenna array elements. The effect of error propagation is investigated as well. All the simulation scenarios have been implemented for two modulation schemes (16-QAM 
and BPSK). The stochastic MIMO channel model has been simulated using both measured ([8]) and theoretical fading correlation model.

With reference to Fig $3-7$ it is obvious that the VMMSE detector performs better than MMSE for any modulation scheme and for any degree of correlation. The effect of error propagation is also investigated for VMMSE. The effect is greater when using 16-QAM modulation. When taking into account correlation between the antenna array elements it is clear that higher correlation leads to degradation of the system performance.

\section{REFERENCES}

[1] D.S. Shiu P.J. Smithi A. Naguib D. Gesbert, M. Shafi. From theory to practice: An overview of mimo spacetime coded wireless systems. IEEE Journal on Selected Areas in Communications., 21(3):281-302, April 2003.

[2] D. Gore D. Gesbert, H. Bolcskei and A. Paulraj. Mimo wireless channels: Capacity and performance prediction. Proceedings of IEEE Globecom Gonference, 2:1083-1088, Nov. 2000.

[3] M.J. Gans D. Chizlik, G.J. Foschini and R.A. Valenzuela. Keyholes, correlations, and capacities of multielement transmit and receive antennas. IEEE Transactions on Wireless Communications, 1(2):78-82, April 2002.

[4] J.P. Kermoal K.I. Pedersen, J.B. Andersen and P. Mogensen. A stochastic multi-input multi-output radio channel model for evaluation of space-time coding algorithms. IEEE VTC Fall 2000, pages 893-897, Sept 2000.

[5] A.F. Molisch. A channel model for mimo systems in macro- and microcellular enviroments. IEEE VTC Spring 2002, 2:655-659, May 2002.

[6] L.E. Telatar. Capacity of multi antenna ganssian channels. ATE'T Bell Labs. and Internal Tech. Memo, june 1995.

[7] D. S, Shiu. Wireless communication using dual antenna array. Norwell: $M A, 2000$.

[8] K.I. Pedersen J.P. Kermoal, L. Schumacher and F. Frederiksen P.E. Mogensen. A stochastic mimo radio channel model with experimental validation. IEEE Journal on Selected Areas in Communications., 20(6):1211-1226, August 2002.

[9] C.K. Rushforth Z. Xic, R.T. Short. A family of suboptimum detectors for coherent multiuser communications. IEEE Journal on Selected Areas in Communications, 8(4):683-690, May 1990.

[10] S. Verdu M. Honig, U. Madhow. Blind adaptive multiuser detection. IEEE Trans. Inform. Theory, 41(4):994-960, Jul. 1995.

[11] G.D. Golden et al. Detection algorithm and initial laboratory results using v _blast spacetime communication architecture. Electron. Lett., 35(1):14-16, January 71999.

[12] C.F. Van Loan G.H. Golub. Matrix computations (Johns Hopkins University Press), 1983.
[13] B.H. Fleury. First- and second-order characterization of direction dispersion and space selectivity in the radio channel. IEEE Thans. Inform. Theory, 46:2027-2044, Sept. 2000.

[14] W.C.Y. Lee. Effects on eorrelation between two mobile radio basestation antennas. IEEE Trans. Commun., 21:1214-1024, Nov. 1973.

[15] J. Salz and J. Winters. Effects of fading correlation on adaptive arrays in digital mobile radio. IEEE Trans. Veh. Technol., 43:1049-1057, Nov. 1994.

[16] J.H. Reed P. Petrus and T.S. Rappaport. Effects of directional antennas at the base station on the doppler spectrum. IEEE Commun. Lett., 1:40-42, Mar. 1997.

[17] A. Lozano F.R. Farrokhi, G.J. Foschini and R.A. Valenznela. Link-optimal space-time processing with multiple transmit and receive antennas. IEEE Commun. Lett., 5(3):85-87, Mar. 2001. 